\title{
Air Monitoring for Volatile Organic Compounds at the Pilot Plant Complex, Aberdeen Proving Ground, Maryland
}

Energy Systems Division Argonne National Laboratory

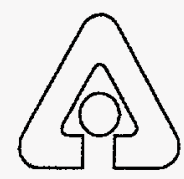

Operated by The University of Chicago,

under Contract W-31-109-Eng-38, for the

United States Department of Energy 


\section{Argonne National Laboratory}

Argonne National Laboratory, with facilities in the states of Illinois and Idaho, is owned by the United States Government, and operated by the University of Chicago under the provisions of a contract with the Department of Energy.

This technical memo is a product of Argonne's Energy Systems (ES) Division. For information on the division's scientific and engineering activities, contact:

Director, Energy Systems Division

Argonne National Laboratory

Argonne, Illinois 60439-4815

Telephone (708) 252-3724

Presented in this technical memo are preliminary results of ongoing work or work that is more limited in scope and depth than that described in formal reports issued by the ES Division.

Publishing support services were provided by Argonne's Information and Publishing Division.

\section{Disclaimer}

This report was prepared as an account of work sponsored by an agency of the United States Government. Neither the United States Government nor any agency thereof, nor any of their employees, makes any warranty, express or implied, or assumes any legal liability or responsibility for the accuracy, completeness, or usefulness of any information, apparatus, product, or process disclosed, or represents that its use would not infringe privately owned rights. Reference herein to any specific commercial product, process, or service by trade name, trademark, manufacturer, or otherwise, does not necessarily constitute or imply its endorsement, recommendation, or favoring by the United States Government or any agency thereof. The views and opinions of authors expressed herein do not necessarily state or reflect those of the United States Government or any agency thereof. 


\section{DISCLAIMER}

Portions of this document may be illegible in electronic image products. Images are produced from the best available original document. 


\section{Air Monitoring for Volatile Organic Compounds at the Pilot Plant Complex, Aberdeen Proving Ground, Maryland}

J.F. Schneider, H.J. O'Neill, L.A. Raphaelian, N.A. Tomczyk, L.F. Sytsma, V.J. Cohut, H.A. Cobo, D.P. O'Reilly, and R.E. Zimmerman

Center for Environmental Restoration Systems, Energy Systems Division,

Argonne National Laboratory, 9700 South Cass Avenue, Argonne, Illinois 60439

March 1995

Work sponsored by United States Department of Defense,

United States Army, Aberdeen Proving Ground, Maryland 


\section{Contents}

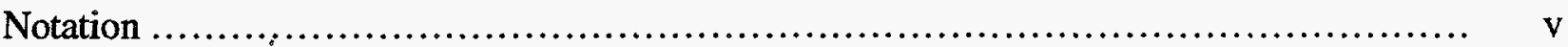

Summary

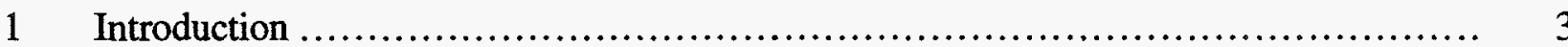

2 Methods and Procedures ....................................................... 6

3 Quality Assurance/Quality Control.............................................. 7

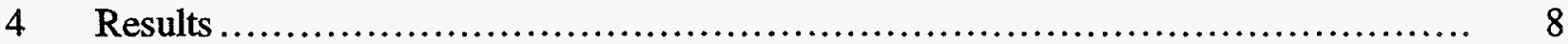

$5 \quad$ Discussion...................................................................... 17

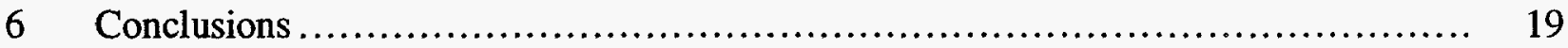

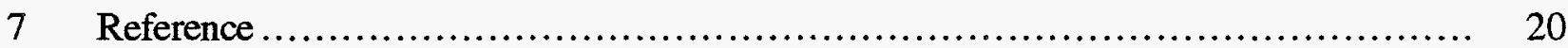

Appendix A: Illinois Institute of Technology Research Institute Report on Analysis of Pilot Plant Complex Air Samples ....................... 21

Appendix B: SciTech Services, Inc., Report on Analysis of Pilot Plant Complex Air Samples....................................... 53

Appendix C: Floor Plans for Buildings Monitored at the Pilot Plant Complex ......... 141

Appendix D: Results of Analysis of Pilot Plant Complex Air Samples by Argonne National Laboratory

\section{Tables}

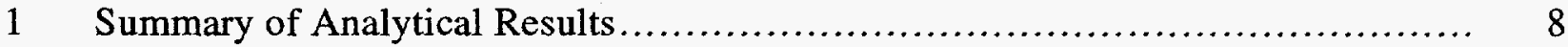

2 Analytical Results for Samples from Outside Building E5625................... 16

\section{Figures}

1 Location of Aberdeen Proving Ground ..................................... 3

2 General Layout of the Pilot Plant Complex ................................ 4

3 Chromatogram for Air Sample PP022 from Room E134 of Building E5625 ..... 18

C-1 Building E5616, Instrument Shop and Change House....................... 143

C-2 Building E5617, Lime House and Pilot Plant Control......................... 144 


\section{Figures (Cont.)}

C-3 Building E5618, Air Compressor House and Plant Neutralization System

C-4 Building E5621, Machine, Pipe, and Carpentry Shop and Inert Gas Compressor House

C-5 Building E5625, First Floor and North Annex............................... 147

C-6 Building E5625, Second Floor and North Annex.............................. 148

C-7 Building E5625, Third Floor and North Annex................................ 149

C-8 Building E5625, Fourth Floor.............................................. 150

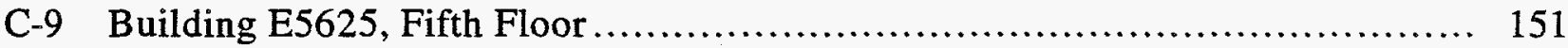

C-10 Building E5626, Guard House and Security Building ....................... 152

C-11 Building E5627, Administration, First Aid, and Quality Control ............... 153

C-12 Building E5632, Refrigerated Storage Building ............................ 154

C-13 Building E5633, Flammable Material Storage ................................ 155 


\section{Notation}

$\begin{array}{ll}\text { ANL } & \text { Argonne National Laboratory } \\ \text { APG } & \text { Aberdeen Proving Ground } \\ \text { CWA } & \text { chemical warfare agent } \\ \text { EA } & \text { Edgewood Area } \\ \text { FPD } & \text { flame photometric detector } \\ \text { GC } & \text { gas chromatograph } \\ \text { ITTRI } & \text { Illinois Institute of Technology Research Institute } \\ \text { L } & \text { liter } \\ \text { L/ } & \text { liters per liter } \\ \text { MS } & \text { mass spectrometer } \\ \text { ng/L } & \text { nanograms per liter } \\ \text { nL/L } & \text { nanoliters per liter } \\ \text { OSHA } & \text { Occupational Safety and Health Administration } \\ \text { PCB } & \text { polychlorinated biphenyl } \\ \text { ppbv } & \text { parts per billion by volume, or } 10^{-9} \mathrm{~L} / \mathrm{L} \\ \text { PPC } & \text { Pilot Plant Complex } \\ \text { pptv } & \text { parts per trillion by volume, or } 10^{-12} \mathrm{~L} / \mathrm{L} \\ \text { RT } & \text { retention time }\end{array}$




\title{
Air Monitoring for Volatile Organic Compounds at the Pilot Plant Complex, Aberdeen Proving Ground, Maryland
}

\author{
by \\ J.F. Schneider, H.J. O'Neill, L.A. Raphaelian, N.A. Tomczyk, \\ L.F. Sytsma, V.J. Cohut, H.A. Cobo, D.P. O'Reilly, and R.E. Zimmerman
}

\section{Summary}

The U.S. Army's Aberdeen Proving Ground has been a test site for a variety of munitions, including chemical warfare agents (CWA). The Pilot Plant Complex (PPC) at Aberdeen was the site of development, manufacture, storage, and disposal of CWA. Deterioration of the buildings and violations of environmental laws led to closure of the complex in 1986. Since that time, all equipment, piping, and conduit in the buildings have been removed. The buildings have been declared free of surface CWA contamination as a result of air sampling using the military system. However, no air sampling has been done to determine if other hazardous volatile organic compounds are present in the PPC, although a wide range of toxic and/or hazardous materials other than CWA was used in the PPC. The assumption has been that the air in the PPC is not hazardous. The purpose of this air-monitoring study was to screen the indoor air in the PPC to confirm the assumption that the air does not contain volatile organic contaminants at levels that would endanger persons in the buildings. A secondary purpose was to identify any potential sources of volatile organic contaminants that need to be monitored in subsequent sampling efforts.

More than 180 air samples from the PPC were sampled and analyzed by Argonne National Laboratory in the fall of 1994 . The majority of the volatile organic compounds found are those compounds commonly present in any building (hydrocarbons and chlorinated solvents). In general, no chemical contaminant was found that contradicts the assumption that the air in the PPC is not hazardous. Polychlorinated biphenyls (PCBs) were identified at low levels (1-50 ng/L). (The OSHA-allowable exposure level for workers is $500 \mathrm{ng} / \mathrm{L}$ [time-weighted average]). Additional sampling for PCBs will be done by taking wipe samples in areas suspected of having $\mathrm{PCB}$ contamination. The results also indicated the presence of organic compounds in the air samples taken in one room (E134 of Building E5625) that are unique to the operations performed in the PPC in the past (for example, 1,4-dithiane and 1,4-oxathiane). 


\section{Introduction}

The U.S. Army's Aberdeen Proving Ground (APG) has been a test site for a variety of munitions, including chemical warfare agents (CWA). At APG, the Pilot Plant Complex (PPC) was the site of development, manufacture, storage, and disposal of CWA. The PPC is located in the Edgewood Area (EA) of Aberdeen, which is situated 21 miles northeast of Baltimore, in the Atlantic Coastal Plain, in an area to the west of Chesapeake Bay (Figure 1). The area in which the PPC is located sits in the Canal Creek basin. The complex, originally designated Complex 87, was constructed in 1941 prior to the entry of the United States into World War II; it contains nine buildings, as shown in Figure 2.

Deterioration of the buildings and violations of environmental laws led to closure of the complex in 1986. Since that time, all equipment, piping, and conduit in the buildings have been removed. The buildings have been declared free of surface CWA contamination as a result of air sampling by using the military system (Lattin 1994). However, no air sampling has been done to determine if other hazardous volatile organic compounds are present in the PPC, although a wide

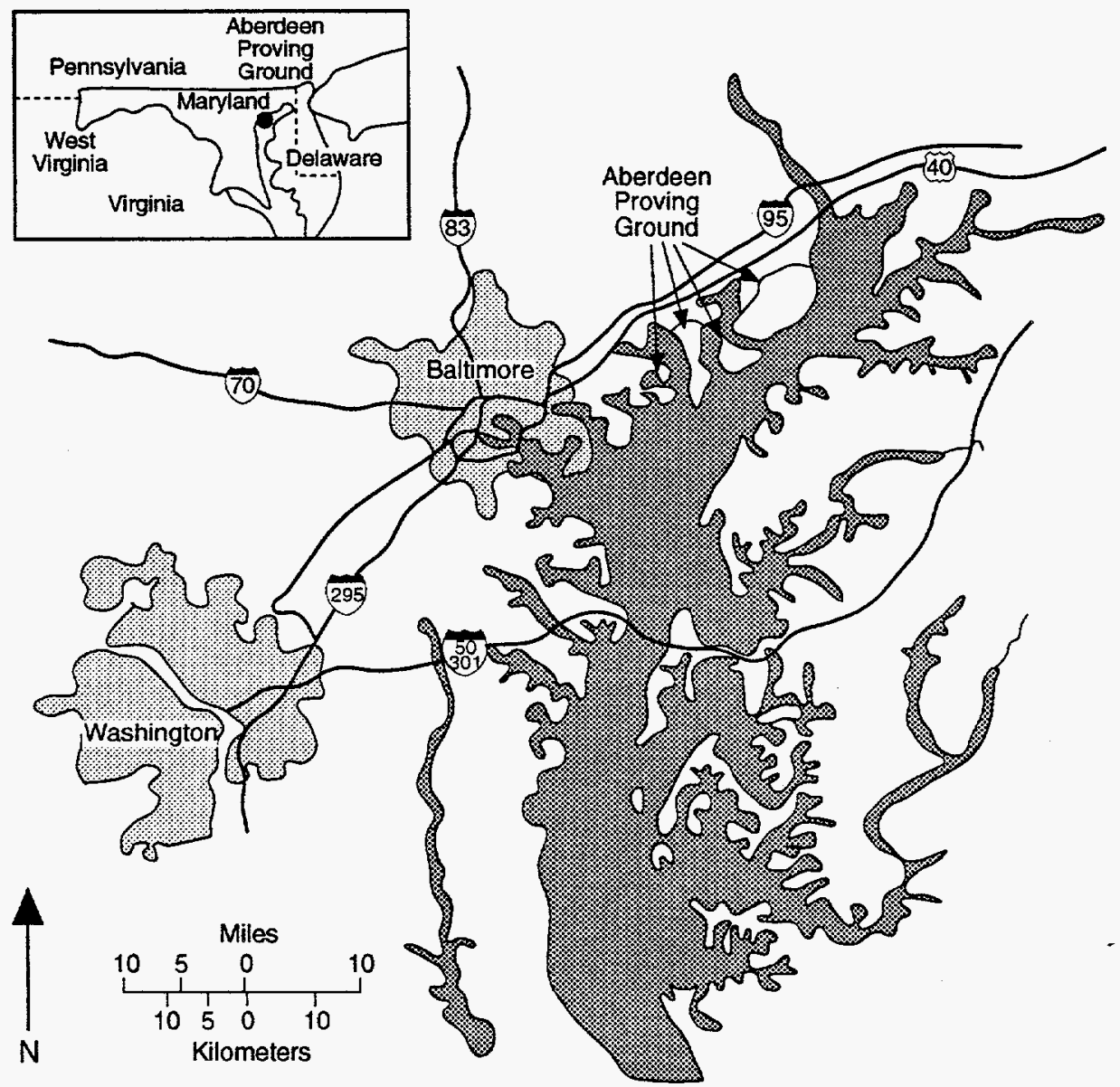

FIGURE 1 Location of Aberdeen Proving Ground 


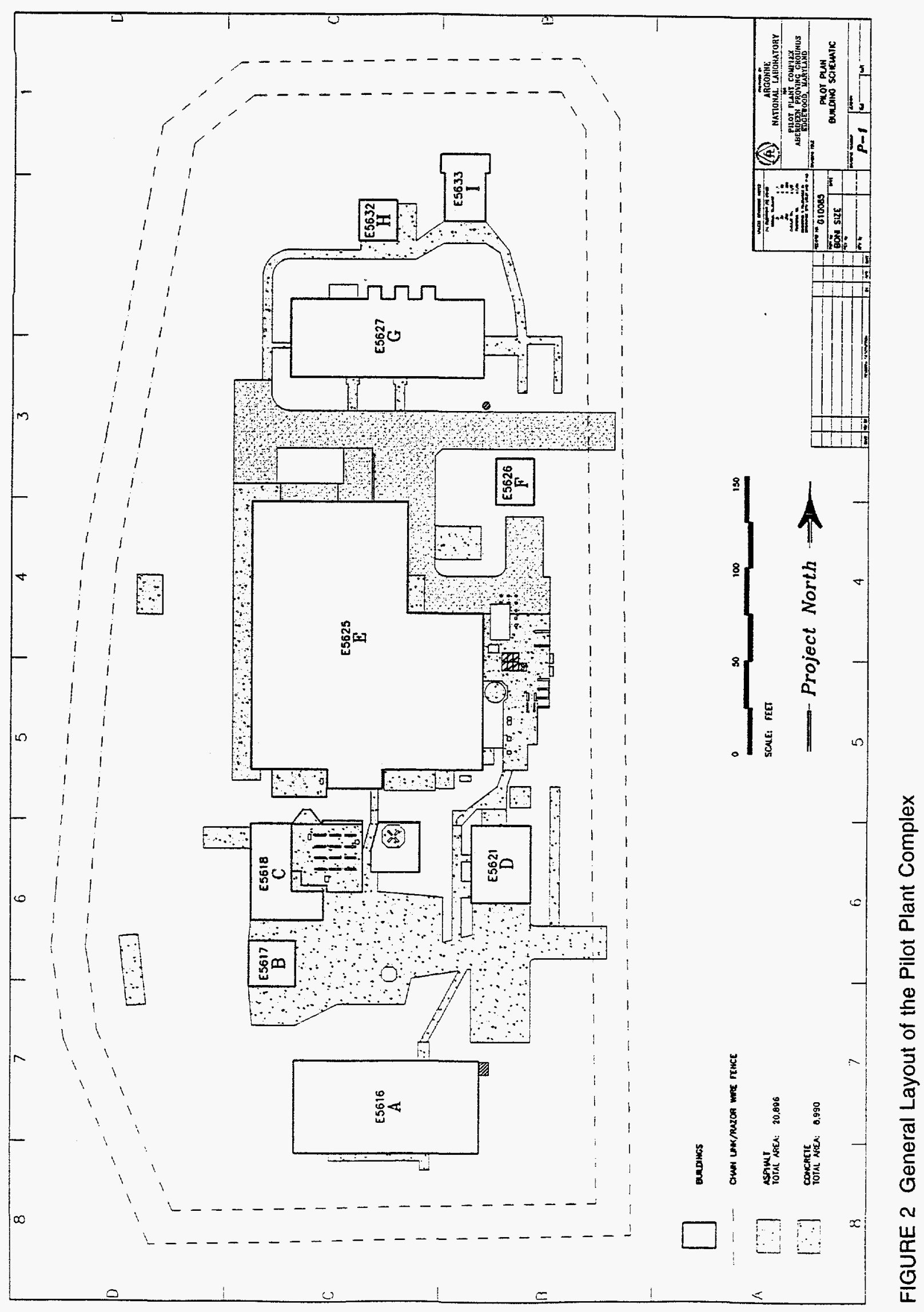


range of toxic and/or hazardous materials other than CWA was used in the PPC. The assumption has been that the air in the PPC is not hazardous.

As part of a program currently being undertaken by Argonne National Laboratory (ANL), the air in the PPC was sampled and analyzed in the fall of 1994. The purpose of this airmonitoring study was to screen the indoor air in the PPC to confirm the assumption that the air does not contain volatile organic contaminants at levels that would endanger persons in the building. A secondary purpose was to identify any potential sources of volatile organic contaminants that need to be monitored in subsequent sampling efforts. The air monitoring discussed in this report is the first phase of a study undertaken to evaluate the condition of the PPC.

The initial air-monitoring effort involved a preliminary survey of the PPC by the Illinois Institute of Technology Research Institute (IITRI) to determine what ambient contaminants can be detected with sampling and analysis technology using preconcentration sorbent tubes. The IITRI report is presented as Appendix A of this report. ANL then proceeded to systematically survey every room and building within the PPC with their air-monitoring procedures. As part of the ANL survey, air samples were also collected by SciTech Services, Inc., for CWA screening. SciTech Services used procedures employed by the Edgewood Research, Development, and Engineering Center, Air Monitoring Branch. The SciTech report is included as Appendix B.

The scope of the ambient-air-monitoring activity conducted by ANL was to screen virtually every laboratory and room of the PPC to determine a spatial distribution of the various ambient air components. This information will be used to plan more extensive sampling and analysis activities in those areas containing the highest levels of contamination. Of particular importance in the ambient-air screening was the search for degradation products of CWA, which may suggest the presence of residual neat chemical agents, and the detection of such environmental contaminants as polychlorinated biphenyls (PCBs). The results of this ambient-air-monitoring survey are described in this report. 


\section{Methods and Procedures}

Air samples were collected and analyzed on-site by ANL in early fall 1994. A total of 186 air samples were collected at numerous locations in the PPC, while additional samples were collected outside the PPC to serve as control samples. The floor plans for the buildings studied are shown in Appendix C, Figures C.1-C.13. At least one air sample was collected from each room.

Sampling was performed by a two-man team, who wore modified level-D personal protection equipment. A personnel sampling pump, model AIRPRO 6000 manufactured by BIOS International, was used to draw ambient air through Tenax TA sorbent polymer sampling cartridge traps $(4 \mathrm{~mm}$ I.D. $\times 11.5 \mathrm{~cm}$ ) at the rate of $500 \mathrm{~mL}$ for $40 \mathrm{~min}$, yielding a $20-\mathrm{L}$ sample volume. The pump was calibrated by using a flow-through meter at the beginning of each sample run and checking it again at the end of the run. The cartridges were analyzed by thermally desorbing the trapped organic compounds, by means of a Dynatherm model 900 ACEM thermal desorption unit, onto a Hewlett-Packard 5890 series II gas chromatograph (GC) equipped with a HewlettPackard 5972 mass spectrometer (MS) and flame photometric detector (FPD). The FPD was specific for sulfur- or phosphorous-containing compounds.

In the Dynatherm, carrier gas was used to sweep away any moisture trapped in the sample cartridge. Then the cartridge was heated to high temperature to desorb the compounds, which were collected on a focusing trap. The focusing trap concentrated the compounds in a small volume for introduction onto the capillary GC column in a narrow band. The GC column was a J\&W DB- 5 column, $30 \mathrm{~m} \times 0.32 \mathrm{~mm}$ I.D. The outlet was split, with $50 \%$ of the flow going to the MS and $50 \%$ going to the FPD.

The MS was used for detecting and identifying organic compounds desorbed from the Tenax traps. A standard mixture of volatile organics was run daily to ensure that the instrument was calibrated properly. Spectra were obtained by scanning from 45 to 400 atomic mass units at a rate of two scans per second. Identifications were made on the basis of mass spectral interpretation and computer searching of the 140,000-peak Wiley spectral library. All quantitations are estimates, using the assumption that analyte response factors are similar to that of toluene in the standard mixture of volatile organics.

Because of the history of the PPC in the production and storage of CWA, and because CWA and CWA degradation products contain sulfur and/or phosphorous, the FPD was used to screen for organic compounds containing sulfur or phosphorous atoms. This information was also useful in identifying unknown organic compounds. Appendix D contains the data obtained by ANL during the on-site screening. 


\section{Quality Assurance/Quality Control}

The purpose of the indoor-air screening performed by ANL was to confirm that the air in the PPC does not contain volatile organic contaminants at levels that would endanger persons in the buildings. A secondary purpose was to identify potential sources of volatile organic contaminants that may need to be monitored in subsequent sampling efforts. This type of screening, classified as level 2, is considered by the U.S. Environmental Protection Agency to be appropriate for determining areas of contamination at a site. If contamination is found, additional sampling at a higher level of quality assurance/quality control is required for confirmation.

The analytical equipment used (GC/MS/FPD) was calibrated daily by using a certified standard mixture supplied by Supelco. The MS was tuned by using perfluorotributyl amine at the maximum sensitivity autotune. Since the purpose of this survey was for screening, no analytes were targeted.

All quantitations were made by assuming the response factor for a compound to be similar to the response factor for toluene, which was run in the certified standard mixture. One-point calibration was done, assuming the GC/MS to be linear over the concentration range of interest. Standard mixtures were run daily.

Identification of the spectral data was accomplished as follows. Appropriate settings or parameters for integrating all but the tiniest peaks and noise in a typical chromatogram were determined experimentally. The peaks then were integrated, and the Wiley library of more than 140,000 peaks was searched by computer. The ANL team examined the Wiley library assignments of structure for each peak and decided, using retention time data, whether any of the library assignments were correct. If the assignment appeared correct, the concentration of the compound was calculated. If the team thought the assignment was incorrect, they examined the mass spectrum and, when possible, made an assignment by using such techniques as looking at the side of the peak to remove impurities, stepping through successive spectra to see if a pure spectrum could be found, or performing background subtraction or subtraction of an interfering peak. 


\section{Results}

The results of the air-monitoring study of the PPC have been summarized on a room-byroom basis. This summary appears in Table 1. Results of analysis of three samples from outside Building E5625 are shown in Table 2. A compilation of all the screening data by building and room number is given in Appendix D.

TABLE 1 Summary of Analytical Results

\begin{tabular}{|c|c|c|c|}
\hline Room & $\begin{array}{l}\text { Sample } \\
\text { Number }\end{array}$ & $\begin{array}{l}\text { Sampling } \\
\text { Location }\end{array}$ & Findings \\
\hline
\end{tabular}

\section{Building E5625}

\begin{tabular}{|c|c|c|c|}
\hline E103 & PP006 & $\begin{array}{l}\text { Middle of back half of the } \\
\text { room }\end{array}$ & No significant findings \\
\hline E104 & PP004 & $\begin{array}{l}\text { Middle of the south half of } \\
\text { the room }\end{array}$ & PCBs at $0.1 \mathrm{~nL} / \mathrm{L}$ \\
\hline E104 & PP005 & $\begin{array}{l}\text { Middle of the north half of } \\
\text { the room }\end{array}$ & PCBs at $2.3 \mathrm{~nL} / \mathrm{L}$ \\
\hline E104 & PP148 & & PCBs at $1.20 \mathrm{~nL} / \mathrm{L}$ \\
\hline E104 & PP149 & & PCBs at $0.70 \mathrm{~nL} / \mathrm{L}$ \\
\hline E104 & PP179 & & PCBs at $0.50 \mathrm{~nL} / \mathrm{L}$ \\
\hline E105 & PP007 & Middle of the room & No significant findings \\
\hline E105 & PP139 & Middle of the room & No significant findings \\
\hline E107 & PP008 & Middle of the room & No significant findings \\
\hline E108 & PP009 & $\begin{array}{l}\text { Middle of the south half of } \\
\text { the room }\end{array}$ & No significant findings \\
\hline E113 & PP013 & Middle of the room & $\begin{array}{l}\text { Unidentified peak with } \mathrm{RT}=20.41 \mathrm{~min} \text { of a } \\
\text { sulfur-containing compound at sub-ppbv } \\
\text { level }\end{array}$ \\
\hline E115 & PP012 & Middle of the room & PCBs at $1 \mathrm{~nL} / \mathrm{L}$ \\
\hline E116 & PP011 & Middle of the room & $\begin{array}{l}\text { PCBs at } 5.7 \mathrm{~nL} / \mathrm{L} \\
\text { Benzothiazole at sub-ppbv level } \\
\text { Unidentified peak with RT }=20.41 \mathrm{~min} \text { of a } \\
\text { sulfur-containing compound at sub-ppbv } \\
\text { level }\end{array}$ \\
\hline
\end{tabular}


TABLE 1 (Cont.)

\begin{tabular}{|c|c|c|c|}
\hline Room & $\begin{array}{l}\text { Sample } \\
\text { Number }\end{array}$ & $\begin{array}{l}\text { Sampling } \\
\text { Location }\end{array}$ & Findings \\
\hline E116 & PP153 & & PCBs at $17.7 \mathrm{~nL} / \mathrm{L}$ \\
\hline E117 & PP010 & Middle of the room & $\begin{array}{l}\text { Unidentified peak with RT }=20.41 \mathrm{~min} \text { of a } \\
\text { sulfur-containing compound at sub-ppbv } \\
\text { level }\end{array}$ \\
\hline E118 & PP014 & Middle of the room & No significant findings \\
\hline E120 & PP020 & Middle of the elevator & No significant findings \\
\hline E121 & PP015 & Middle of the room & No significant findings \\
\hline E122 & PP019 & Middle of the room & No significant findings \\
\hline E123 & PP017 & $\begin{array}{l}\text { Middle of the north half of } \\
\text { the room }\end{array}$ & PCBs at $9.8 \mathrm{~nL} / \mathrm{L}$ \\
\hline E123 & PP018 & $\begin{array}{l}\text { In front of the elevator } \\
\text { shaft in the south half of } \\
\text { the room }\end{array}$ & No significant findings \\
\hline E124 & PP016 & Middle of the room & No significant findings \\
\hline E128 & PP028 & Middle of the room & No significant findings \\
\hline E128 & PP029 & $\begin{array}{l}\text { Middle of the room as a } \\
\text { duplicate of PP028 }\end{array}$ & No significant findings \\
\hline E129 & PP025 & $\begin{array}{l}\text { Middle of the east half of } \\
\text { the room }\end{array}$ & No significant findings \\
\hline E131 & PP023 & $\begin{array}{l}\text { Near the sump in the north } \\
\text { half of the room }\end{array}$ & $\begin{array}{l}\text { 1,4-oxathiane at sub-ppbv level } \\
\text { Unknown sulfur-containing compound with } \\
\mathrm{RT}=15.41 \mathrm{~min} \text { at sub-ppbv level } \\
\text { O,O-diethyl-S-ethyl phosphorothioate at } \\
\text { sub-ppbv level } \\
\text { Phosphoric acid triethyl ester at } 0.53 \mathrm{~nL} / \mathrm{L}\end{array}$ \\
\hline E131 & PP026 & $\begin{array}{l}\text { Middle of the south end of } \\
\text { the hallway }\end{array}$ & $\begin{array}{l}\text { 1,4-oxathiane at sub-ppbv level } \\
\text { PCBs at } 7.3 \mathrm{~nL} / \mathrm{L}\end{array}$ \\
\hline E131 & PP124 & $\begin{array}{l}\text { South side of the room } \\
\text { as a duplicate of PP026 }\end{array}$ & $\begin{array}{l}\text { Diisopropylamine is the largest peak } \\
(\sim 1 \mathrm{ng} / \mathrm{L}) \\
\text { Oxathiane at sub-ppbv level } \\
\text { No PCBS present in repeat of sample PP026 }\end{array}$ \\
\hline
\end{tabular}


TABLE 1 (Cont.)

\begin{tabular}{|c|c|c|c|}
\hline Room & $\begin{array}{l}\text { Sample } \\
\text { Number }\end{array}$ & $\begin{array}{l}\text { Sampling } \\
\text { Location }\end{array}$ & Findings \\
\hline E132 & PP021 & Middle of the room & $\begin{array}{l}\text { 1,4-oxathiane at sub-ppbv level } \\
\text { Unknown sulfur-containing compound with } \\
\text { RT }=15.41 \text { min at sub-ppbv level } \\
\text { O,O-diethyl-S-ethyl phosphorothioate at } \\
\text { sub-ppbv level }\end{array}$ \\
\hline E134 & $\begin{array}{l}\text { PP001 } \\
\text { PP002 }\end{array}$ & $\begin{array}{l}\text { Southeast section } \\
\text { Northwest section }\end{array}$ & $\begin{array}{l}\text { 1,4-oxathiane at }-0.5 \mathrm{~nL} / \mathrm{L} \\
1,4 \text {-dithiane at sub-ppbv level } \\
\text { O,O-diethyl-S-ethyl phosphorothioate at } \\
\text { sub-ppbv level } \\
\text { Benzothiazole at sub-ppbv level } \\
\text { Unknown sulfur-containing compounds with } \\
\text { RTs of } 11.91,6.38,17.11 \text {, and } \\
17.37 \text { min at sub-ppbv levels }\end{array}$ \\
\hline E134 & PP022 & $\begin{array}{l}\text { Middle of the room; } \\
\text { sampled for } 100 \mathrm{~min} \\
\text { for a total volume of } \\
50 \mathrm{~L}\end{array}$ & $\begin{array}{l}\text { 1,4-oxathiane at sub-ppbv level } \\
\text { 1,4-dithiane at sub-ppbv level } \\
\text { O,O-diethyl-S-ethyl phosphorothioate at } \\
\text { sub-ppbv level } \\
\text { Benzothiazole at sub-ppbv level } \\
\text { Unknown sulfur-containing compounds with } \\
\text { RTs of } 11.91,16.38,17.11 \text {, and } \\
17.37 \text { min at sub-ppbv levels }\end{array}$ \\
\hline E135 & PP024 & $\begin{array}{l}\text { In room E129 at the } \\
\text { opening to room E135 }\end{array}$ & $\begin{array}{l}\text { O,O-diethyl-S-ethyl phosphorothioate at } \\
\text { sub-ppbv level } \\
\text { Unknown sulfur-containing compound with } \\
\text { RT }=18.28 \mathrm{~min} \text { at sub-ppbv level }\end{array}$ \\
\hline E201 & PP046 & & PCBs at $\sim 0.7 \mathrm{~nL} / \mathrm{L}$ \\
\hline E203 & $\left.\begin{array}{l}\text { PP032 } \\
\text { PP033 }\end{array}\right\}$ & & $\begin{array}{l}\text { PCBs at } \sim 0.5 \mathrm{~nL} / \mathrm{L} \\
\text { Biphenyl and diphenyl ether (components of } \\
\text { Dowtherm) are the compounds present in } \\
\text { the greatest amount. } \\
\text { Naphthalene at } 0.2 \mathrm{~nL} / \mathrm{L}\end{array}$ \\
\hline $\mathrm{E} 204$ & $\begin{array}{l}\text { PP030 } \\
\text { PP031 }\end{array}$ & & $\begin{array}{l}\text { Findings similar to Room E203 except no } \\
\text { PCBs }\end{array}$ \\
\hline E205 & PP036 & & $\begin{array}{l}\text { 1,4-oxathiane at sub-ppbv level } \\
\text { PCBs at } \sim 0.4 \mathrm{~nL} / \mathrm{L}\end{array}$ \\
\hline E207 & PP037 & & $\begin{array}{l}\text { PCBs } 8.1 \mathrm{~nL} / \mathrm{L} ; 20 \text { different congeners } \\
\text { identified } \\
\text { Dowtherm components also found }\end{array}$ \\
\hline
\end{tabular}


TABLE 1 (Cont.)

\begin{tabular}{|c|c|c|c|}
\hline Room & $\begin{array}{l}\text { Sample } \\
\text { Number }\end{array}$ & $\begin{array}{l}\text { Sampling } \\
\text { Location }\end{array}$ & Findings \\
\hline E208 & $\left.\begin{array}{l}\text { PP034 } \\
\text { PP035 }\end{array}\right\}$ & & PCBs at $\sim 0.2 \mathrm{~nL} / \mathrm{L}$ \\
\hline E209 & PP038 & & $\begin{array}{l}\text { No significant findings but more aldehydes } \\
\text { and ketones than most samples; e.g., } \\
\text { acetophenone }=0.11 \mathrm{~nL} / \mathrm{L} \\
\text { benzaldehyde }=0.16 \mathrm{~nL} / \mathrm{L}\end{array}$ \\
\hline E211 & PP039 & & Findings similar to room E209 \\
\hline E213 & PP042 & & PCBs at $\sim 0.3 \mathrm{~nL} / \mathrm{L}$ \\
\hline E214 & PP043 & & PCBs at $\sim 0.2 \mathrm{~nL} / \mathrm{L}$ \\
\hline E215 & PP044 & & PCBs at $-1 \mathrm{~nL} / \mathrm{L}$ \\
\hline E216 & $\left.\begin{array}{l}\text { PP040 } \\
\text { PP041 }\end{array}\right\}$ & & No significant findings \\
\hline E217 & PP045 & & $\begin{array}{l}\text { Aromatic hydrocarbons are higher than } \\
\text { usual } \\
\text { PCBs at } \sim 0.4 \mathrm{~nL} / \mathrm{L}\end{array}$ \\
\hline E220 & PP047 & Southeast corner & PCBs at $11.9 \mathrm{~nL} / \mathrm{L}$ \\
\hline E220 & PP048 & Southwest & PCBs at $1.80 \mathrm{~nL} / \mathrm{L}$ \\
\hline E220 & PP049 & West & PCBs at 0 \\
\hline E220 & PP050 & Northwest & PCBs at $1.10 \mathrm{~nL} / \mathrm{L}$ \\
\hline E301 & PP052 & & PCBs at $\sim 0.2 \mathrm{~nL} / \mathrm{L}$ \\
\hline E303 & $\begin{array}{l}\text { PP056 } \\
\text { PP057 }\end{array}$ & & No significant findings \\
\hline E305 & PP054 & South side of room & Trace PCBs \\
\hline E305 & PP055 & North side of room & PCBs at $4.9 \mathrm{~nL} / \mathrm{L}$ \\
\hline E306 & PP053 & & PCBs at $\sim 0.05 \mathrm{~nL} / \mathrm{L}$ \\
\hline E308 & $\left.\begin{array}{l}\text { PP060 } \\
\text { PP061 }\end{array}\right\}$ & & $\begin{array}{l}\text { Higher volatile content than nearby rooms; } \\
\text { mainly aromatics }\end{array}$ \\
\hline
\end{tabular}


TABLE 1 (Cont.)

\begin{tabular}{|c|c|c|c|}
\hline Room & $\begin{array}{l}\text { Sample } \\
\text { Number }\end{array}$ & $\begin{array}{l}\text { Sampling } \\
\text { Location }\end{array}$ & Findings \\
\hline E309 & $\left.\begin{array}{l}\text { PP058 } \\
\text { PP059 }\end{array}\right\}$ & & No significant findings \\
\hline E310 & PP063 & & Aromatic content totaling $\sim 15 \mathrm{ng} / \mathrm{L}$ \\
\hline E311 & PP062 & South & No significant findings \\
\hline E311 & PP064 & & Aromatic content of $\sim 15 \mathrm{ng} / \mathrm{L}$ \\
\hline E313 & PP065 & & No significant findings \\
\hline E314 & PP066 & North & PCBs at $\sim 3 \mathrm{~nL} / \mathrm{L}$ \\
\hline E314 & PP067 & South & No significant findings \\
\hline E317 & PP073 & Center & No significant findings \\
\hline E318 & PP071 & Center & No significant findings \\
\hline E319 & PP070 & Center & No significant findings \\
\hline E320 & PP069 & Center & No significant findings \\
\hline E403 & PP078 & North & No significant findings \\
\hline E403 & PP079 & South & No significant findings \\
\hline E405 & PP074 & Northeast & No significant findings \\
\hline E405 & PP075 & Southwest & No significant findings \\
\hline$E 408$ & PP076 & Northeast & No significant findings \\
\hline E408 & PP077 & Southwest & No significant findings \\
\hline E409 & PP086R & West & PCBs at $\sim 1 \mathrm{~nL} / \mathrm{L}$ \\
\hline E409 & PP085 & East & No significant findings \\
\hline E411 & PP084 & Doorway & No significant findings \\
\hline E412 & PP080 & North & No significant findings \\
\hline $\mathrm{E} 412$ & PP081 & South & No significant findings \\
\hline
\end{tabular}


TABLE 1 (Cont.)

\begin{tabular}{|c|c|c|c|}
\hline Room & $\begin{array}{l}\text { Sample } \\
\text { Number }\end{array}$ & $\begin{array}{l}\text { Sampling } \\
\text { Location }\end{array}$ & Findings \\
\hline E415 & PP090 & Center & $\begin{array}{l}\text { Unidentified sulfur-containing peak at } \\
\mathrm{RT}=20.52 \mathrm{~min} \\
\mathrm{PCBs} \text { at } \sim 0.4 \mathrm{~nL} / \mathrm{L}\end{array}$ \\
\hline E416 & PP089R & Center & $\begin{array}{l}\text { Unidentified sulfur-containing peaks at RTs } \\
\text { of } 18.49 \text { and } 20.52 \mathrm{~min} \\
\text { PCBs at } \sim 0.5 \mathrm{~nL} / \mathrm{L}\end{array}$ \\
\hline E416 & PP166 & & PCBs at $1.60 \mathrm{~nL} / \mathrm{L}$ \\
\hline E417 & PP088 & Center & No significant findings \\
\hline E418 & PP087 & Center & $\begin{array}{l}\text { Unidentified sulfur-containing peak at } \\
\mathrm{RT}=20.52 \mathrm{~min} \\
\mathrm{PCBs} \text { at } \sim 0.2 \mathrm{~nL} / \mathrm{L}\end{array}$ \\
\hline $\begin{array}{l}\text { Elevator } \\
\text { mechanical } \\
\text { room }\end{array}$ & PP125 & - & Ethyl hexanol at $0.47 \mathrm{~nL} / \mathrm{L}$ \\
\hline \multicolumn{4}{|c|}{ Samples analyzed with $P$ filter on FPD } \\
\hline E133 & & & Nothing on FPD-P filter \\
\hline E134 & & & Nothing on FPD-P filter \\
\hline \multicolumn{4}{|c|}{ Building 5625 Sumps } \\
\hline A 104 & PP 122 & West sump & $\begin{array}{l}\text { TCE }(\sim 4 \mathrm{ng} / \mathrm{L}) \text { and PCE }(\sim 2.3 \mathrm{ng} / \mathrm{L}) \text { are } \\
\text { only compounds over } 1 \mathrm{ng} / \mathrm{L}\end{array}$ \\
\hline A116 & PP121 & South wall sump & $\begin{array}{l}\text { Many compounds over } 1 \mathrm{ng} / \mathrm{L} \text { in this sample } \\
\text { Largest peak is aniline }(\sim 120 \mathrm{ng} / \mathrm{L}) \text {; next } \\
\text { highest is diisopropylamine }(\sim 85 \mathrm{ng} / \mathrm{L}) \\
\text { Many chlorinated organic compounds } \\
\text { Perhaps }>200 \text { compounds in this sample; } \\
24 \text { peaks appear in FPD (oxathiane and } \\
\text { some disulfides identified) }\end{array}$ \\
\hline A 131 & PP123 & North sump & $\begin{array}{l}\text { Many brominated and chlorinated } \\
\text { compounds; possible chloroacetophenone } \\
\text { at } \sim 0.1 \mathrm{ng} / \mathrm{L} ; \text { trichlorobenzene is largest } \\
\text { peak at } \sim 2 \mathrm{ng} / \mathrm{L}\end{array}$ \\
\hline
\end{tabular}


TABLE 1 (Cont.)

$\begin{array}{lll}\text { Room } & \begin{array}{c}\text { Sample } \\ \text { Number }\end{array} & \begin{array}{l}\text { Sampling } \\ \text { Location }\end{array}\end{array}$

\section{Building E5616}

$\left.\begin{array}{ll}\text { A107 } & \text { PP114 } \\ & \text { PP116 } \\ \text { PP117 } \\ \text { PP118 }\end{array}\right\}$

A 109

PP120

A110

PP119

\section{Building E5617}

B101

PP106

\section{Building E5618}

C101

PP093

C101

PP173

C102

PP100

PP101

$C_{103}$

PP102

PP103

C103

PP170

C104

PP104

PP105

C104

PP171
Southeast

North sump

Center

Middle

$\left.\begin{array}{l}\text { Northeast } \\ \text { Southwest }\end{array}\right\}$

$\left.\begin{array}{l}\text { Northeast } \\ \text { Southwest }\end{array}\right\}$

Southwest

$\left.\begin{array}{l}\text { Northeast } \\ \text { Southwest }\end{array}\right\}$

Northeast
Findings
Naphthalene was the largest peak in each of the four samples; only naphthalene was $>1 \mathrm{ng} / \mathrm{L}$ (in SW corner)

PCBs ranged from $O$ in NW and NE comers to $0.4 \mathrm{~nL} / \mathrm{L}$ in $S W$ and $0.3 \mathrm{~nL} / \mathrm{L}$ in SE corners

Cyclohexanone at $\sim 0.5 \mathrm{ng} / \mathrm{L}$ (did not appear in other samples)

Large unknown peak identified as octene around $\mathrm{RT}=30 \mathrm{~min}$

No significant findings

No significant findings other than PCBs at $\sim 0.5 \mathrm{~nL} / \mathrm{L}$

Benzothiophene at $\sim 1.5 \mathrm{ng} / \mathrm{L}$ Naphthalene level at $0.76 \mathrm{~nL} / \mathrm{L}$ PCBs at $1.2 \mathrm{~nL} / \mathrm{L}$

PCBs at $18.8 \mathrm{~nL} / \mathrm{L}$

Naphthalene at $\sim 4 \mathrm{ng} / \mathrm{L}$, substituted naphthalene at $\sim 2 \mathrm{ng} / \mathrm{L}$

PCBs at $5.5 \mathrm{~nL} / \mathrm{L}$ in both samples

Naphthalene at $\sim 4 \mathrm{ng} / \mathrm{L}$, substituted naphthalene at $\sim 1 \mathrm{ng} / \mathrm{L}$

PCBs at $3.8 \mathrm{~nL} / \mathrm{L}$

Concentrations slightly lower in sample PP103

Benzothiophene found in PP102 (both MS and FPD)

PCBs at $\sim 47 \mathrm{~nL} / \mathrm{L}$

Naphthalene at $\sim 1 \mathrm{ng} / \mathrm{L}$

PCBs at $1.4 \mathrm{~nL} / \mathrm{L}$

Slightly higher concentrations in sample PP105 than sample PP104

PCBs at $\sim 50 \mathrm{~nL} / \mathrm{L}$ 
TABLE 1 (Cont.)

\begin{tabular}{llll}
\hline Room & $\begin{array}{c}\text { Sample } \\
\text { Number }\end{array}$ & $\begin{array}{l}\text { Sampling } \\
\text { Location }\end{array}$ & Findings \\
\hline
\end{tabular}

Building E5621

\begin{tabular}{|c|c|c|c|}
\hline D101 & PP108 & Northeast sump & $\begin{array}{l}\text { Many higher aliphatic hydrocarbons but } \\
\text { nothing significant } \\
\text { PCBs at } \sim 0.2 \mathrm{~nL} / \mathrm{L}\end{array}$ \\
\hline D101 & PP109 & $10 \mathrm{ft}$ south of sump & $\begin{array}{l}\text { Pinene at }-2 \mathrm{ng} / \mathrm{L} \\
\text { Naphthalene at } \sim 1.4 \mathrm{ng} / \mathrm{L} \\
\text { Methyl naphthalene at } \sim 4 \mathrm{ng} / \mathrm{L} \\
\text { Benzothiophene at }-0.3 \mathrm{ng} / \mathrm{L} \\
\text { PCBs at } \sim 0.2 \mathrm{~nL} / \mathrm{L}\end{array}$ \\
\hline D101 & PP112 & $\begin{array}{l}\text { West wall next to } \\
\text { stained concrete }\end{array}$ & $\begin{array}{l}\text { Pinene at } \sim 1.5 \mathrm{ng} / \mathrm{L} \\
\text { Naphthalene at } \sim 3.8 \mathrm{ng} / \mathrm{L} \\
\text { Methyl naphthalene at } \sim 2 \mathrm{ng} / \mathrm{L} \\
\text { Dimethyl naphthalene at } \sim 1.5 \mathrm{ng} / \mathrm{L} \\
\text { Phenanthrene at } \sim 1 \mathrm{ng} / \mathrm{L} \text {. } \\
\text { Many aliphatic and aromatic hydrocarbons } \\
\text { PCBs at } \sim 0.6 \mathrm{~nL} / \mathrm{L}\end{array}$ \\
\hline D102 & PP111 & & PCBs at $\sim 0.06 \mathrm{~nL} / \mathrm{L}$ \\
\hline D103 & PP094 & Center & Benzothiophene at $\sim 7.5 \mathrm{ng} / \mathrm{L}$. \\
\hline D103 & PP110 & & $\begin{array}{l}\text { Naphthalene at } \sim 10 \mathrm{ng} / \mathrm{L} \\
\text { Methyl naphthalene at } \sim 4 \mathrm{ng} / \mathrm{L} \\
\text { Dimethyl naphthalene at } \sim 2 \mathrm{ng} / \mathrm{L} \\
\text { Many aliphatic hydrocarbons }\end{array}$ \\
\hline D104 & PP113 & & No significant findings \\
\hline
\end{tabular}

Building E5626

F104 PP083

Center

No significant findings

\section{Building E5627}

$\begin{array}{llll}\text { G110 } & \text { PP098 } & \text { Center } & \text { No significant findings } \\ \text { G118 } & \text { PP096 } & \text { Center } & \text { No significant findings } \\ \text { G120 } & \text { PP095 } & \text { West } & \text { No significant findings } \\ \text { G120 } & \text { PP097 } & \text { East } & \text { No significant findings }\end{array}$


TABLE 1 (Cont.)

\begin{tabular}{|c|c|c|c|}
\hline Room & $\begin{array}{l}\text { Sample } \\
\text { Number }\end{array}$ & $\begin{array}{l}\text { Sampling } \\
\text { Location }\end{array}$ & Findings \\
\hline \multicolumn{4}{|c|}{ Building E5633 } \\
\hline 1101 & PP091 & Center & No significant findings \\
\hline
\end{tabular}

TABLE 2 Analytical Results for Samples from Outside Building E5625

\begin{tabular}{llll}
\hline $\begin{array}{c}\text { Sampling } \\
\text { Date }\end{array}$ & $\begin{array}{c}\text { Sample } \\
\text { Number }\end{array}$ & $\begin{array}{c}\text { Sampling } \\
\text { Location }\end{array}$ & Findings \\
\hline $8-10-94$ & PP072 & North & No significant findings \\
$8-11-94$ & PP082 & North & No significant findings \\
$8-12-94$ & PP092 & North & No significant findings \\
\hline
\end{tabular}




\section{Discussion}

The majority of the volatile organic compounds found (hydrocarbons and chlorinated solvents) during the PPC air monitoring by ANL are compounds commonly found in any building. No CWA were found at detectable levels, but the CWA-related compounds 1,4-dithiane and 1,4-oxathiane were found in room E134 of Building E5625.

In the analysis of a sample from room E134 of Building E5625, an unknown peak with a retention time (RT) of 18.327 min was detected on the sulfur channel of the GC/FPD. This RT is similar to that of bis (2-chloroethyl) sulfide (mustard). The unknown compound was estimated to be present at a concentration ( 0.1 part per trillion volume [pptv]) below the level detectable by MS. To further investigate the unknown CWA found in room E134, an air sample was collected for $300 \mathrm{~min}$ at a rate of $500 \mathrm{~mL}$ of air per minute. The sample volume was $150 \mathrm{~L}$. Figure 3 is the FPD chromatogram of this sample, showing peaks for 1,4-dithiane $(\mathrm{RT}=16.421 \mathrm{~min}$; concentration of $\sim 0.17 \mathrm{pptv}$ or $\left.10^{-12} \mathrm{~L} / \mathrm{L}\right)$ and 1,4 -oxathiane (RT $=12.822$, : concentration of $\sim 0.45 \mathrm{pptv}$ or $10^{-12} \mathrm{~L} / \mathrm{L}$ ), and the unknown peak. Assuming that the response for bis (2-chloroethyl) sulfide is similar to that for 1,4-dithiane, the limit of detection for bis (2-chloroethyl) sulfide is estimated to be approximately 100 parts per quadrillion volume $\left(10^{-15} \mathrm{~L} / \mathrm{L}\right)$ for this volume of sample $(150 \mathrm{~L})$.

Over 150 compounds have been identified in the various samples. Many other compounds are part of isomeric categories and are not listed individually. At least 20 different congeners of PCBs are collectively called PCBs. The PCB data was utilized to identify those locations within the PPC that will require more extensive sampling for PCB contamination. This activity will involve the analysis of wipe samples from specific rooms and buildings within the PPC to determine if PCB contamination exceeds regulatory limits. 


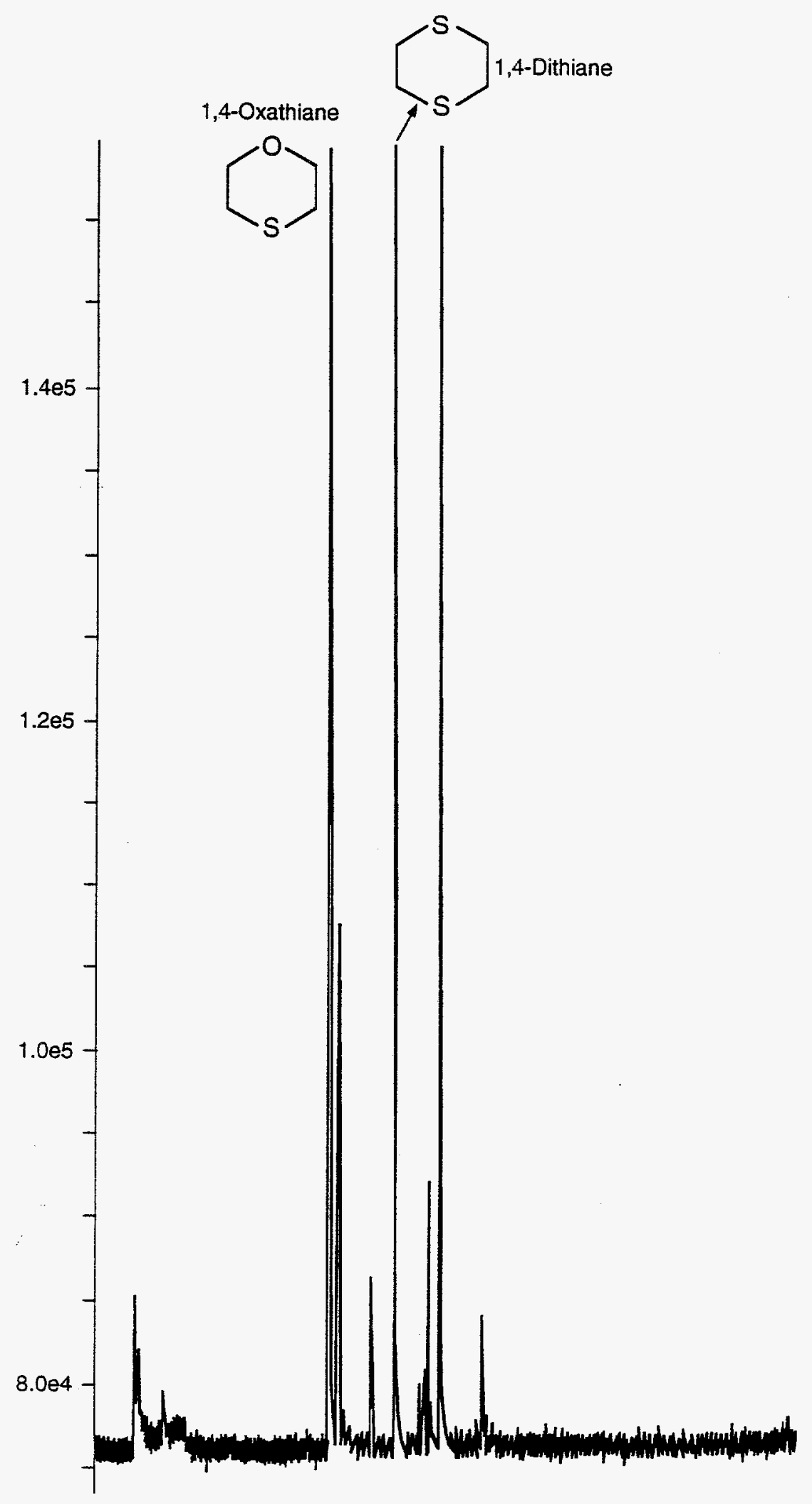

FIGURE 3 Chromatogram for Air Sample PP022 from Room E134 of Building E5625 


\section{Conclusions}

Over 180 air samples from the buildings of the PPC were analyzed by ANL. The majority of the volatile organic compounds found (hydrocarbons and chlorinated solvents) are commonly present in any building. In general, no chemical contaminant was found that would contradict the assumption that the air in the PPC is not hazardous. The findings in the IITRI report (Appendix A) and the SciTech report (Appendix B) are in agreement with the ANL work. PCBs were identified at low levels (1-50 ng/L). (The OSHA-allowable PCB exposure level for workers is $500 \mathrm{ng} / \mathrm{L}$ [time-weighted average]). Additional sampling for PCBs will be done by taking wipe samples in areas suspected of having PCB contamination. The results also indicated the presence of organic compounds in the air that are unique to the operations performed in the PPC in the past (for example, 1,4 dithiane and 1,4 oxathiane). This information will be useful in determining if additional sampling of the PPC is required and, if so, where in the PPC such sampling should be done.

It should be emphasized that the ambient-air sampling of the PPC only allows the evaluation of the presence of CWAs and other toxic compounds that are volatile under ambient conditions. The PPC will have to be surveyed by other techniques for the nonvolatile analytes of concern to ensure their absence from the facility prior to demolition. 


\section{Reference}

Lattin, F.G., 1994, unpublished information, Edgewood Research and Development Engineering Center, Aberdeen Proving Ground, Md. 
Appendix A:

Illinois Institute of Technology Research Institute Report on Analysis of Pilot Plant Complex Air Samples 


\title{
BROAD SPECTRUM GC/MS ANALYSES \\ ON COLLECTED TENAX CARTRIDGES
}

\author{
Submitted to: \\ University of Chicago-Argonne National Laboratory \\ 9700 South Cass Avenue \\ Argonne, Illinois 60439-4873
}

\author{
By: \\ Michael Miller \\ IIT Research Institute \\ 10 West 35th Street \\ Chicago, Illinois 60616
}

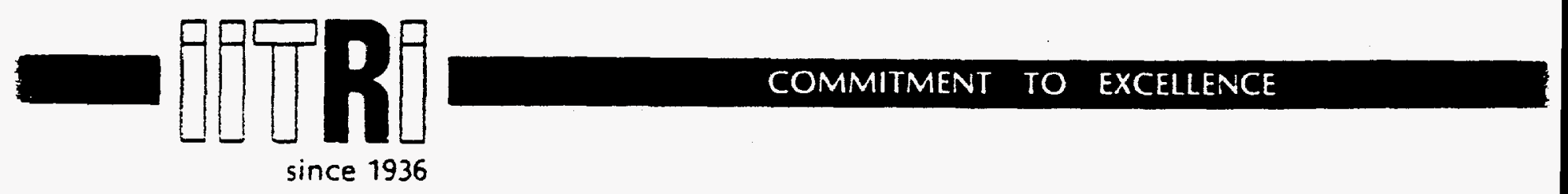




\section{VOLATILE ORGANIC COMPOUNDS ON TENAX}

\section{ANALYTICAL PROCEDURE}

\section{Sample Collection}

The volatile organics present in the gas-phase emissions at the APG Pilot Plant sampling locations were collected by Argonne personnel using Tenax-GR cartridges and sampling equipment supplied by IITRI. Clean glass cartridges $(10 \mathrm{~cm} \times 1.5 \mathrm{~cm}$ i.d.) were packed with approximately 2 grams of 60-80 mesh Tenax-GR which was held in place using clean glass wool plugs. The packed cartridges then were thermally-conditioned at $285^{\circ} \mathrm{C}$ under constant flow of helium gas for four (4) hours. These conditioned cartridges were sealed in clean Teflon-lined screw-cap culture tubes inside sealed metal cans and stored cold until use. Machined Teflon plugs were used to isolate and immobilize the Tenax cartridges within the cultures tubes in order to reduce contamination and the risk of breakage during transport.

Two Tenax-GR samples were collected at each sampling site - one 20-liter and one 10liter. The reason for sampling at two different air volumes was to provide a "range-finding" sample in each case. If the first sample saturated the detector of the mass spectrometer, the experimental conditions could be suitably adjusted to give a successful analysis using the second sample.

The volatile organic compounds (VOCs) were collected by attaching the Tenax cartridge to the suction side of a pump, positioning the collector at the sampling location and drawing the required volume through it. A calibrated flowmeter was used to monitor the flowrate (500 cc/min), which was controlled with a valve. The cartridges that were used for sample collection and then analyzed by GC/MS are identified in Table 1 .

\section{Sample Transfer and GC/MS Analysis}

Characterization and quantification of the VOCs were accomplished by thermal desorption and purging of the Tenax-GR cartridges with helium into a liquid-nitrogen cooled nickel 
Table 1. Tenax Sample Identification

\begin{tabular}{|c|c|c|c|c|}
\hline $\begin{array}{c}\text { Sample } \\
\text { Location }\end{array}$ & $\begin{array}{c}\text { Cartridge } \\
\text { Number }\end{array}$ & $\begin{array}{c}\text { Collection } \\
\text { Date }\end{array}$ & $\begin{array}{c}\text { Sample } \\
\text { Volume, (L) }\end{array}$ & $\begin{array}{c}\text { GC/MS } \\
\text { File Name }\end{array}$ \\
\hline 113 & TG2-25 & $6 / 22$ & 20.0 & ANL11320 \\
\hline 2D2 & TG2-01 & $6 / 22$ & -20.0 & ANL2D220 \\
\hline 3D2 & TG2-10 & $6 / 22$ & 20.0 & ANL3D220 \\
\hline 4D2 & TG2-04 & $6 / 22$ & 20.0 & ANL4D220 \\
\hline
\end{tabular}

capillary trap (using a Tekmar $5010 \mathrm{GT}$ thermal desorber). After an 8 minute desorption at $285^{\circ} \mathrm{C}$, the cryotrap was rapidly heated to $250^{\circ} \mathrm{C}$ and the compounds flushed with helium onto a high-resolution capillary gas chromatographic column, to separate the components. Identification and quantification were achieved by mass spectrometric measurement of the total ion current signal, followed by computer enhancement of the raw GC/MS data.

Samples were analyzed on a $60 \mathrm{~m} \times 0.32 \mathrm{~mm}$ i.d. DB1701 chemically-bonded fused-silica capillary column, with a carrier gas flow rate of $-1.0 \mathrm{ml} / \mathrm{min}$. The column was initially held at a temperature of $35^{\circ} \mathrm{C}$ for 5 minutes, then heated at $4^{\circ} \mathrm{C} / \mathrm{min}$ to $220^{\circ} \mathrm{C}$. The column was coupled directly to the ion source of the mass spectrometer, a Finnigan MAT 44S quadrupole instrument, which was cyclically scanned from $\mathrm{m} / \mathrm{z} 29$ to $\mathrm{m} / \mathrm{z} 300$ every $1.5 \mathrm{sec}$ during each run. The data were acquired and stored using a Zenith personal computer.

\section{Data Enhancement and Spectrum Identification}

Clean mass spectra were extracted from the raw GC/MS data by application of a dataenhancement algorithm. ${ }^{1}$ This program produces clean spectra automatically by subtracting background contributions and resolving overlapping peaks. Identification of the resolved spectra was established using two computer-based mass spectral library search systems and by manual comparison of the unknown spectra with a compendium of standard spectra.

1 R. G. Dromey, M. J. Stefik, T. C. Rindfleisch, and A. M. Duffield, Anal. Chem. 48, 1368 (1976). 


\section{Semi-Quantitative Estimates of Compound Concentrations}

The intensity of response is given by the areas of the individual peaks in the GC/MS profiles, and is related to the actual concentration of the compounds present. A standard was used to convert peak areas to estimates of the actual amounts of each chemical in the sample. For this study, toluene was used as the standard. A known quantity of toluene was loaded onto a Tenax-GR cartridge and used to generate a response under standard GC/MS operating conditions. The relationship between the amount of toluene analyzed and its peak area response was used to estimate the amount of each compound in the sample. Division of this amount by the volume of air sampled gave a semi-quantitative estimate of the concentration (ng/L) of the component. These values were converted into parts-per-billion by volume (ppbv) by taking into account the molecular' weight of each identified compound. Compounds vary in response within the analytical system (GC/MS) and therefore the accuracy of these values is dependent upon the similarity of each compound's response with that of toluene.

\section{RESULTS AND DISCUSSION}

The total ion current profiles obtained by GC/MS analysis of the air samples listed in Table 1 are shown in Figures $1-4$. (The $\mathrm{x}$-axis represents the number of acquired scans ( $1.5 \mathrm{sec} / \mathrm{scan})$ and the $y$-axis the component intensity, in arbitrary units.) These plots represent the volatile organic compounds present in the gas-phase at the sampling locations. Using procedures described above, the GC/MS peaks were identified, and semi-quantitative estimates of their concentrations were determined. These results are summarized in Tables $2-5$. The data, which have been re-arranged according to compound class, are presented in Tables $6-9$.

A field or trip-blank also was analyzed in order to access possible contamination from the field or the storage conditions. The total ion current profile and analytical results for this blank are presented in Figure 5 and Tables 10 and 11, respectively. 


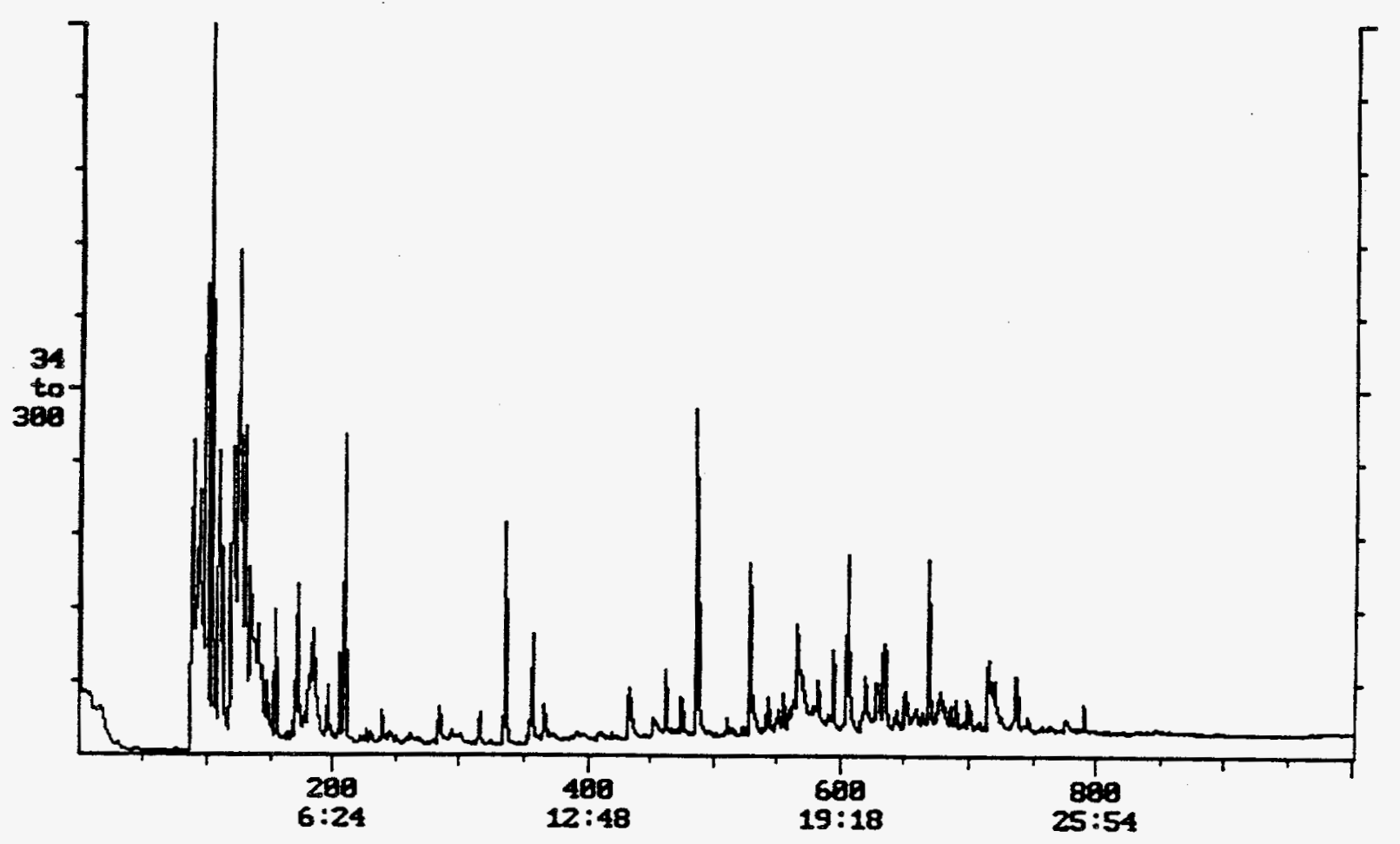

Figure 1. Total Ion Current Profile From the GC/MS Analysis of the Air Sample Collected in the APG Pilot Plant at the 113 Location. 


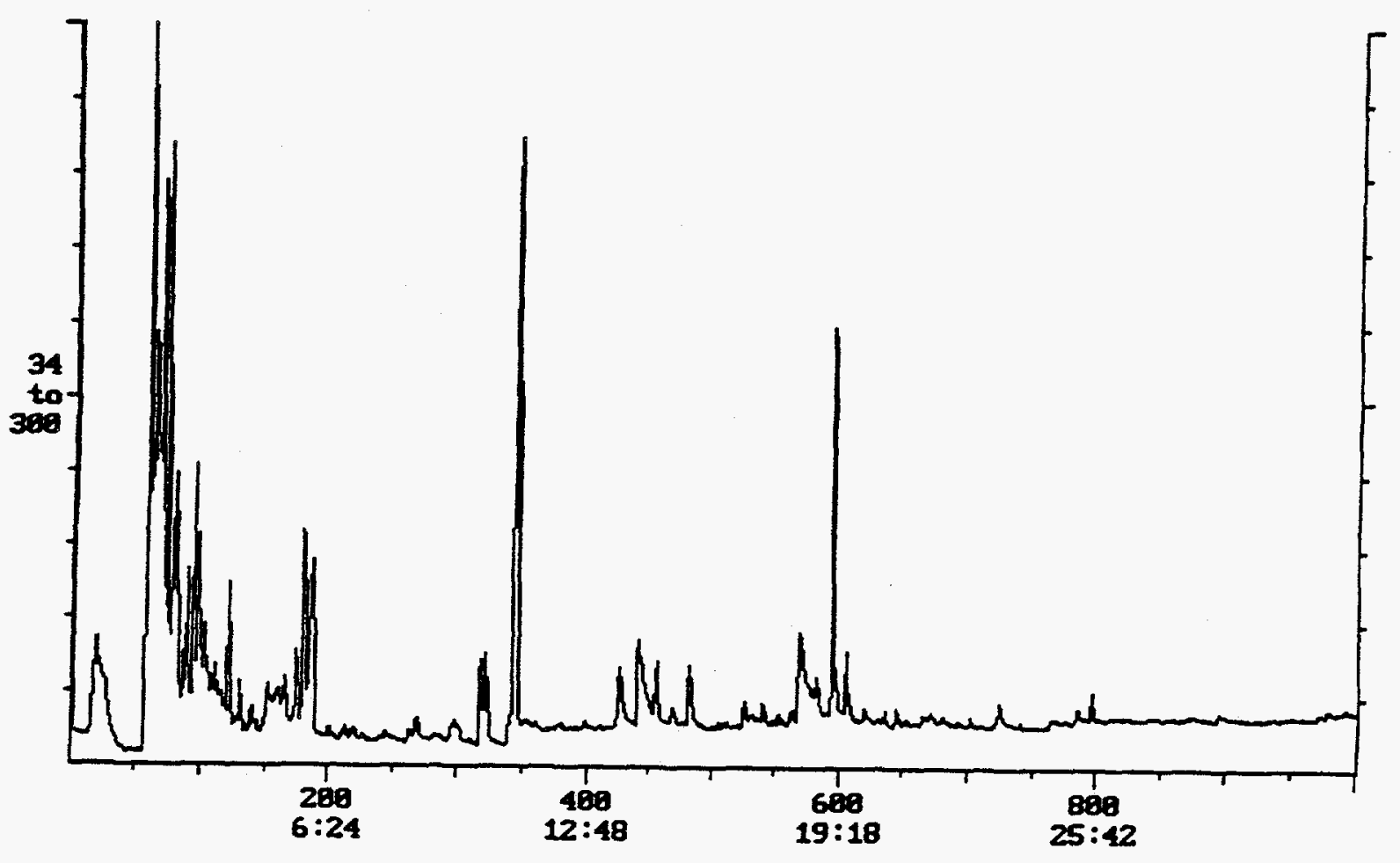

Figure 2. Total Ion Current Profile From the GC/MS Analysis of the Air Sample Collected in the APG Pilot Plant at the 2D2 Location. 


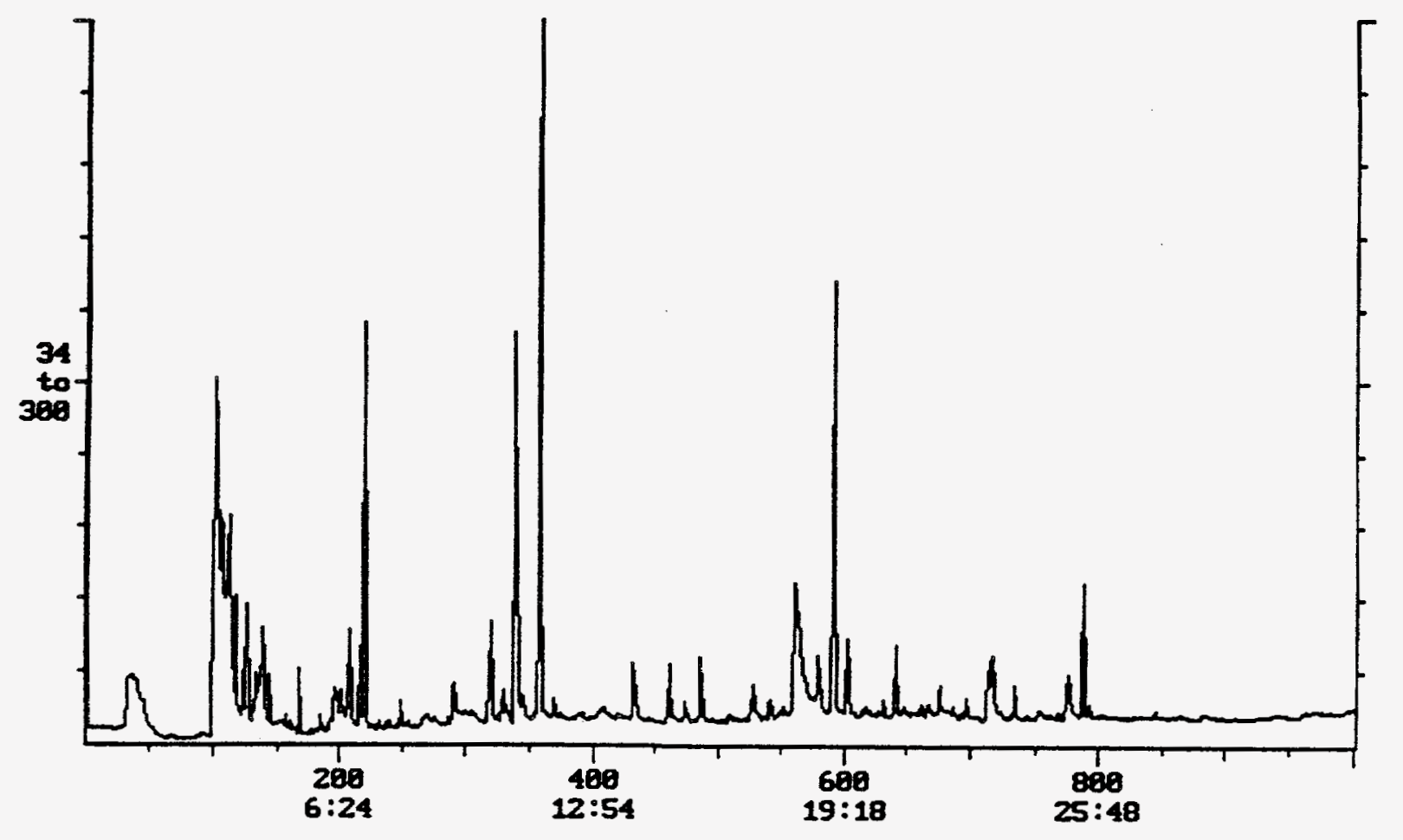

Figure 3. Total Ion Current Profile From the GC/MS Analysis of the Air Sample Collected in the APG Pilot Plant at the 3D2 Location. 


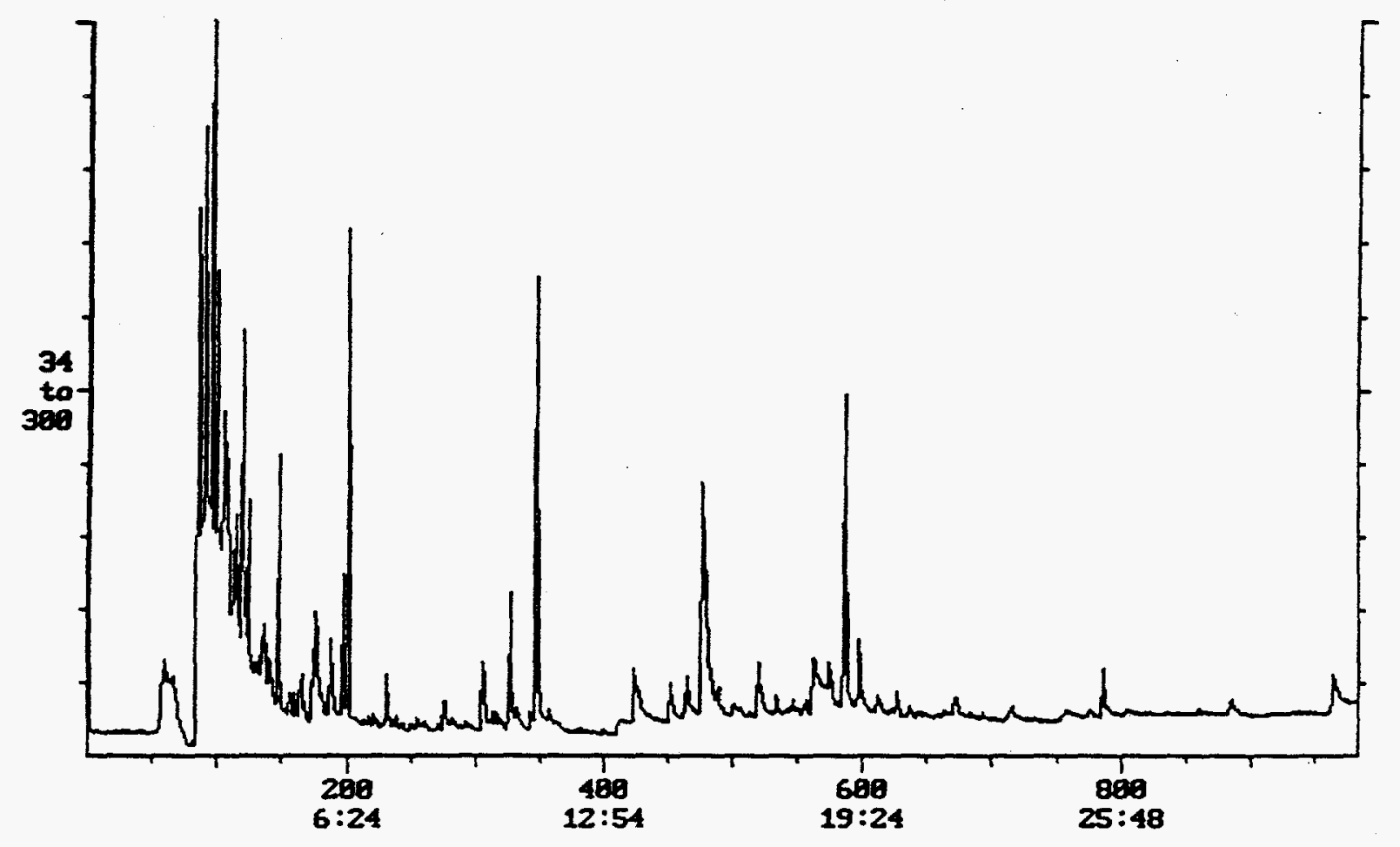

Figure 4. Total Ion Current Profile From the GC/MS Analysis of the Air Sample Collected in the APG Pilot Plant at the 4D2 Location. 
Table 2. Air Monitoring Result Determined from the Tenax-GR Sample Collected in the APG Pibt Plant at the 1/3 Location

\begin{tabular}{|c|c|c|c|c|c|c|}
\hline \multirow[b]{2}{*}{$\begin{array}{l}\text { Spectrum } \\
\text { Number }\end{array}$} & \multicolumn{5}{|c|}{ Sample Code : TG2-25 } & \multirow[b]{2}{*}{$\begin{array}{l}\text { Amount } \\
\text { (ng) }\end{array}$} \\
\hline & Formula & MW & Compound & $\frac{\text { Conce }}{\text { (ng/L) }}$ & $\frac{\text { tration }}{\text { (ppby) }}$ & \\
\hline $\begin{array}{l}94 \\
100 \\
104 \\
104 \\
110 \\
112 \\
113 \\
118 \\
119 \\
121 \\
125 \\
130 \\
131 \\
134 \\
141 \\
143 \\
143 \\
146 \\
148 \\
154 \\
164 \\
170 \\
171 \\
172 \\
178 \\
181 \\
183 \\
185 \\
189 \\
196 \\
205 \\
226 \\
230 \\
240 \\
245 \\
284 \\
284 \\
294 \\
301 \\
316 \\
316 \\
335 \\
352 \\
366 \\
372 \\
409 \\
412 \\
433 \\
462 \\
\end{array}$ & 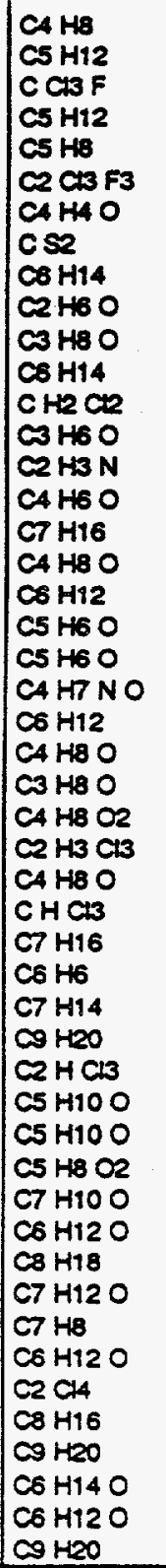 & $\begin{array}{r}56 \\
72 \\
136 \\
72 \\
68 \\
186 \\
68 \\
76 \\
86 \\
46 \\
58 \\
86 \\
84 \\
60 \\
41 \\
70 \\
100 \\
72 \\
84 \\
82 \\
82 \\
85 \\
84 \\
72 \\
60 \\
88 \\
132 \\
72 \\
118 \\
100 \\
78 \\
98 \\
128 \\
130 \\
86 \\
86 \\
100 \\
110 \\
100 \\
114 \\
112 \\
92 \\
100 \\
164 \\
112 \\
128 \\
102 \\
100 \\
128 \\
\end{array}$ & 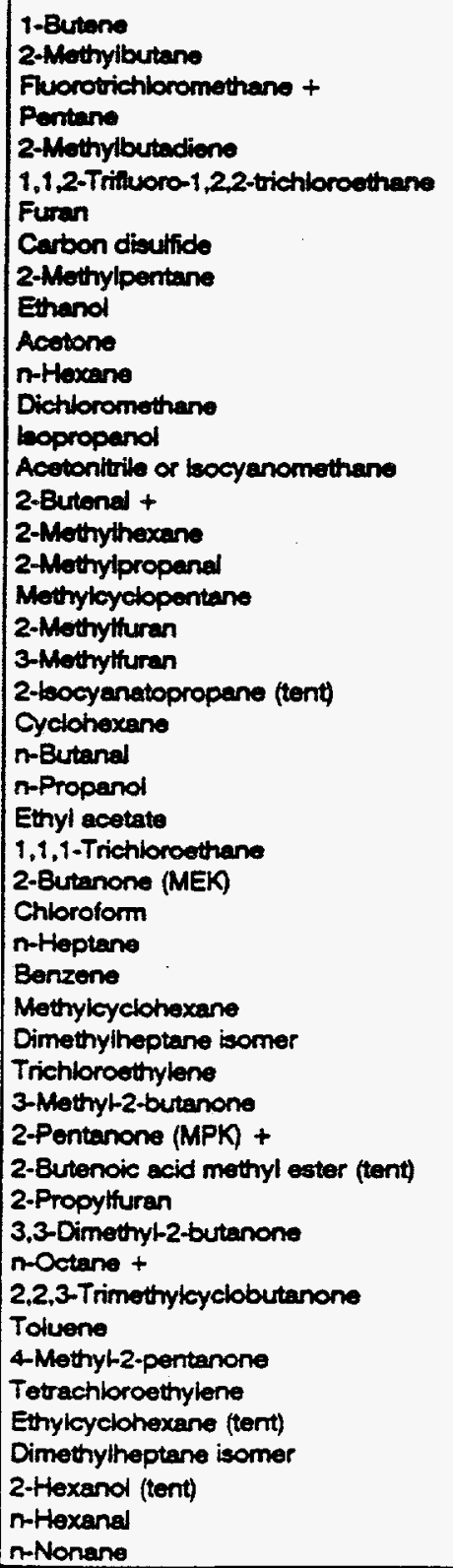 & $\begin{array}{r}0.3 \\
6.5 \\
9.1 \\
0.5 \\
0.3 \\
0.5 \\
0.1 \\
0.3 \\
4.3 \\
14 \\
0.7 \\
0.3 \\
<0.1 \\
0.2 \\
0.2 \\
0.1 \\
0.1 \\
0.1 \\
0.4 \\
<0.1 \\
<0.1 \\
0.2 \\
0.5 \\
0.3 \\
0.1 \\
0.1 \\
0.2 \\
0.1 \\
0.4 \\
0.3 \\
<0.3\end{array}$ & $\begin{array}{r}0.1 \\
2.0 \\
1.5 \\
0.2 \\
<0.1 \\
0.1 \\
<0.1 \\
0.1 \\
2.1 \\
5.5 \\
0.2 \\
0.1 \\
<0.1 \\
0.1 \\
0.1 \\
<0.1 \\
<00.1 \\
<0.1 \\
0.1 \\
<0.1 \\
<0.1 \\
0.1 \\
0.2 \\
0.1 \\
<0.1 \\
<0.1 \\
<0.1 \\
0.1 \\
<0.1 \\
0.1 \\
0.1 \\
0.1 \\
<0.1 \\
<00.1 \\
<0.1 \\
<0.1 \\
<0.1 \\
<0.1 \\
<0.1 \\
<0.1 \\
<0.1 \\
<0.1 \\
<0.1 \\
<0.1 \\
<0.1 \\
<0.1 \\
0.3 \\
0.1 \\
0.1 \\
0.1\end{array}$ & $\begin{array}{r}6.9 \\
130 \\
182 \\
\\
10 \\
5.5 \\
9.1 \\
1.5 \\
5.0 \\
86 \\
284 \\
13 \\
6.4 \\
400 \\
4.5 \\
3.6 \\
\\
1.2 \\
2.4 \\
8.7 \\
0.6 \\
1.1 \\
4.1 \\
10 \\
6.1 \\
2.6 \\
4.5 \\
6.8 \\
0.8 \\
7.0 \\
7.4 \\
1.2 \\
0.6 \\
3.5 \\
1.4 \\
3.5 \\
\\
0.5 \\
0.9 \\
3.2 \\
\\
21 \\
0.7 \\
4.2 \\
0.5 \\
1.2 \\
1.0 \\
7.8 \\
5.3\end{array}$ \\
\hline
\end{tabular}

Concentrations are semi-quantitative estimates determined by comparison to the response for a known ampunt of Toluene. tent = Tentative identification 
Table 2. Air Monitoring Results Determined trom the Tenax-OR Sample Collected in the APQ Pilot Plant at the 113 Location

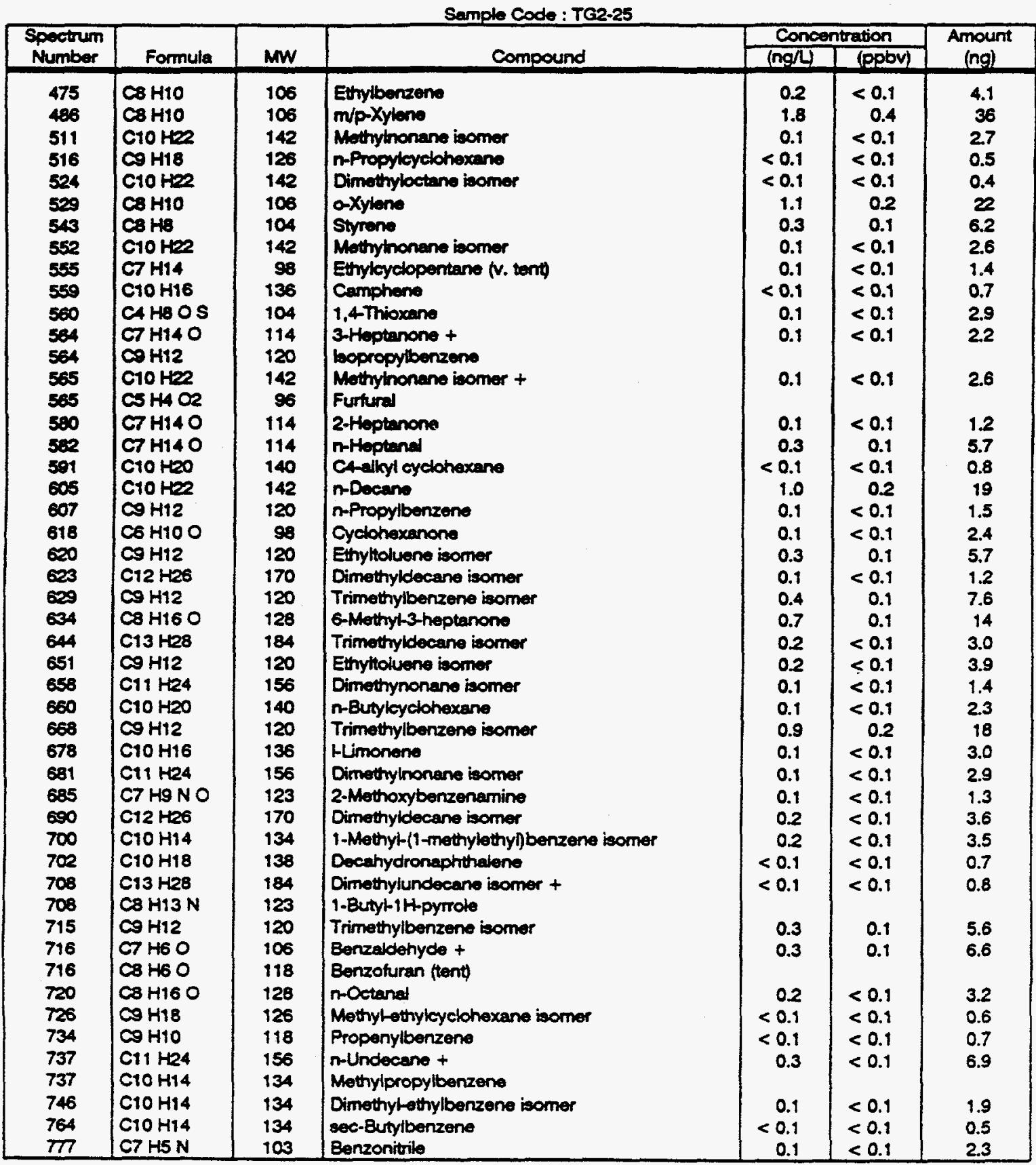

Concentrations are semi-quantitative estimates determined by comparison to the response for a known amount of Toluene. tent = Tentative Idontification

v. tent $=$ Very Tentative Identification 
Table 3. Air Monitoring Reault Dotermined from the Tonax-GR Sample Collocted in the APG Pilot Plant at the 202 Location

Sample Code : TC2-01

\begin{tabular}{|c|c|c|c|c|c|c|}
\hline \multirow{2}{*}{$\begin{array}{l}\text { Spectrum } \\
\text { Number }\end{array}$} & \multirow[b]{2}{*}{ Formula } & \multirow[b]{2}{*}{$\mathbf{M W}$} & \multirow[b]{2}{*}{ Compound } & \multicolumn{2}{|c|}{ Concentration } & \multirow{2}{*}{$\begin{array}{c}\text { Amount } \\
\text { (ng) }\end{array}$} \\
\hline & & & & (ngl) & (ppov) & \\
\hline $\begin{array}{l}61 \\
61 \\
64 \\
68 \\
73 \\
73 \\
74 \\
79 \\
80 \\
82 \\
83 \\
86 \\
87 \\
80 \\
89 \\
94 \\
98 \\
99 \\
109 \\
111 \\
122 \\
131 \\
140 \\
140 \\
152 \\
152 \\
153 \\
160 \\
160 \\
160 \\
165 \\
174 \\
176 \\
213 \\
269 \\
269 \\
298 \\
298 \\
322 \\
426 \\
442 \\
456 \\
469 \\
482\end{array}$ & 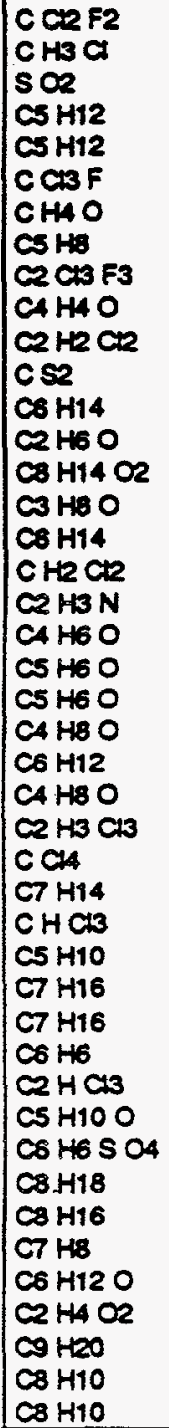 & $\begin{array}{r}120 \\
50 \\
64 \\
72 \\
72 \\
136 \\
32 \\
68 \\
186 \\
68 \\
86 \\
76 \\
86 \\
46 \\
142 \\
58 \\
86 \\
84 \\
41 \\
70 \\
82 \\
82 \\
72 \\
84 \\
72 \\
132 \\
152 \\
98 \\
118 \\
70 \\
100 \\
100 \\
78 \\
130 \\
86 \\
174 \\
114 \\
112 \\
92 \\
100 \\
60 \\
128 \\
106 \\
106\end{array}$ & 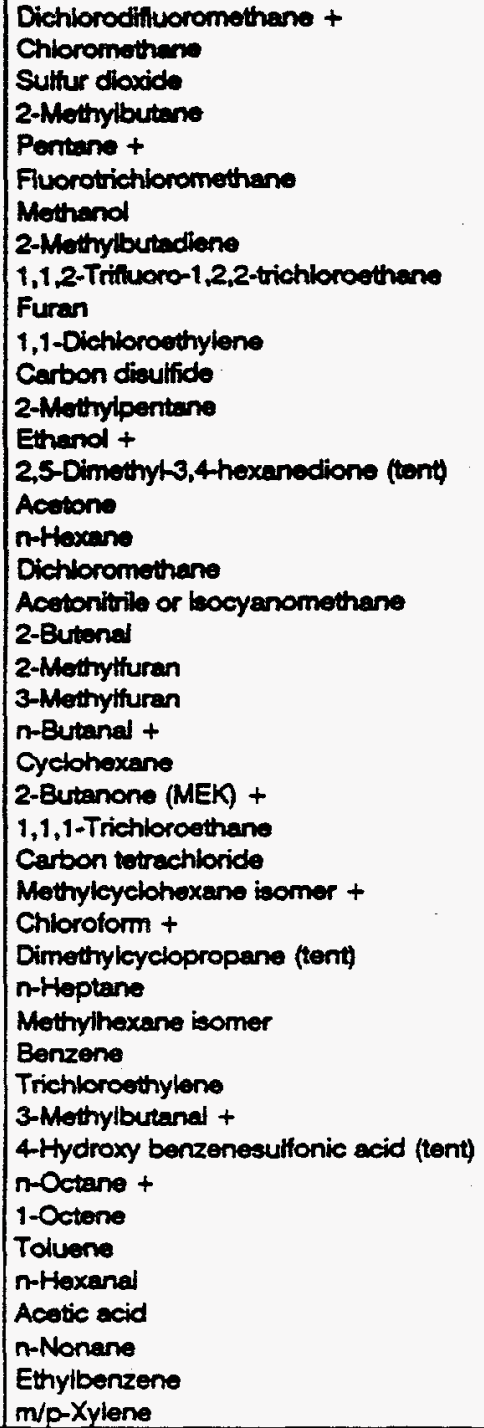 & $\begin{array}{r}0.2 \\
0.1 \\
2.2 \\
0.9 \\
0.2 \\
0.3 \\
0.2 \\
0.4 \\
<0.1 \\
<0.1 \\
0.1 \\
0.5 \\
0.5 \\
0.3 \\
0.1 \\
<0.1 \\
0.1 \\
0.3 \\
0.2 \\
0.1 \\
0.1 \\
<0.1 \\
0.1 \\
\\
0.1 \\
<0.1 \\
0.3 \\
0.1 \\
0.1 \\
0.1 \\
0.1 \\
0.2 \\
0.5 \\
0.2 \\
0.1 \\
0.2\end{array}$ & $\begin{array}{r}<0.1 \\
0.1 \\
0.7 \\
0.3 \\
\\
0.1 \\
0.1 \\
<0.1 \\
0.1 \\
<0.1 \\
<0.1 \\
<0.1 \\
0.2 \\
0.2 \\
0.1 \\
<0.1 \\
<0.1 \\
<0.1 \\
0.1 \\
0.1 \\
<0.1 \\
<0.1 \\
<0.1 \\
<0.1 \\
<0.1 \\
<0 \\
<0.1 \\
<0.1 \\
<0.1 \\
<0.1 \\
<0.1 \\
<0.1 \\
<0.1\end{array}$ & $\begin{array}{r}4.5 \\
3.0 \\
45 \\
17 \\
\\
3.5 \\
6.2 \\
3.5 \\
7.1 \\
0.3 \\
0.9 \\
2.0 \\
9.7 \\
\\
10 \\
6.4 \\
1.8 \\
\text { trece } \\
1.9 \\
5.7 \\
4.9 \\
1.3 \\
\\
1.7 \\
0.6 \\
1.2\end{array}$ \\
\hline
\end{tabular}

Concentrations are semi-quantitative estimates determined by comparison to the response for a known amount of Toluene. tent I Tentative Identification 
Table 3. Air Monitoring Recults Dotermined from the Tenax-GR Sample Collected in the APG Pilot Plant at the 2D2 Location

Sample Code : TG2-01

\begin{tabular}{|c|c|c|c|c|c|c|}
\hline \multirow{2}{*}{$\begin{array}{l}\text { Spectinin } \\
\text { Number }\end{array}$} & \multirow{2}{*}{ Formula } & \multirow[b]{2}{*}{$M W$} & \multirow[b]{2}{*}{ Compound } & \multicolumn{2}{|c|}{ Concentration } & \multirow{2}{*}{$\begin{array}{c}\text { Amount } \\
\text { (ng) }\end{array}$} \\
\hline & & & & (ng/) & (pobv) & \\
\hline $\begin{array}{c}510 \\
507 \\
527 \\
541 \\
568 \\
564 \\
560 \\
582 \\
605 \\
605 \\
645 \\
680 \\
702 \\
724 \\
741 \\
766 \\
786 \\
786 \\
874 \\
894 \\
1013\end{array}$ & 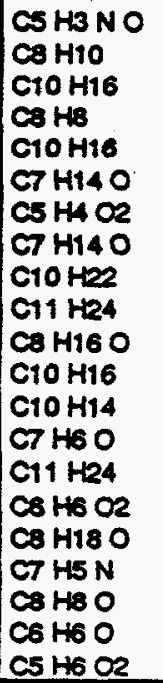 & $\begin{array}{r}93 \\
106 \\
136 \\
104 \\
138 \\
114 \\
96 \\
114 \\
142 \\
156 \\
128 \\
136 \\
134 \\
106 \\
156 \\
110 \\
130 \\
103 \\
120 \\
94 \\
98 \\
\end{array}$ & 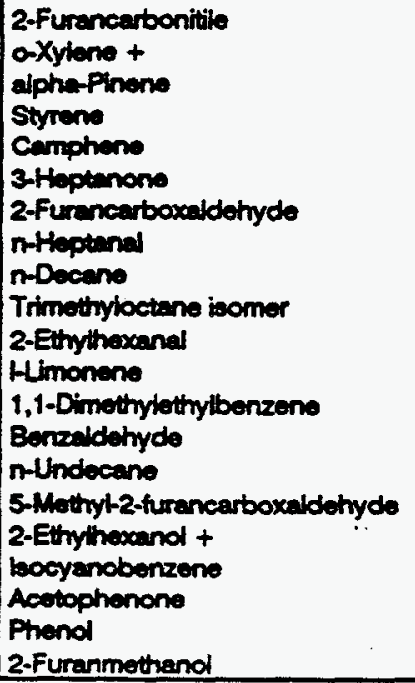 & $\begin{array}{r}<0.1 \\
0.1 \\
\\
0.1 \\
<0.1 \\
<0.1 \\
0.2 \\
0.1 \\
0.2 \\
<0.1 \\
0.1 \\
<0.1 \\
<0.1 \\
0.1 \\
<0.1 \\
<0.1 \\
0.1 \\
0.1 \\
<0.1 \\
<0.1 \\
0.2 \\
\end{array}$ & $\begin{array}{l}<0.1 \\
<0.1 \\
<0.1 \\
<0.1 \\
<0.1 \\
<0.1 \\
<0.1 \\
<0.1 \\
<0.1 \\
<0.1 \\
<0.1 \\
<0.1 \\
<0.1 \\
<0.1 \\
<0.1 \\
<0.1 \\
<0.1 \\
<0.1 \\
<0.1\end{array}$ & $\begin{array}{r}\text { trece } \\
1.9 \\
1.3 \\
\text { trace } \\
0.9 \\
3.9 \\
1.8 \\
3.1 \\
0.7 \\
1.2 \\
\text { trace } \\
\text { trace } \\
1.3 \\
\text { trace } \\
\text { trece } \\
1.0 \\
\text { trece } \\
\text { trece } \\
3.7\end{array}$ \\
\hline
\end{tabular}

Concentrations are semi-quantitative estimates determined by comparison to the response for a known amount of Toluene. tent = Tentative identification 
Teble 4. Air Monitoring Results Determined from the Tonax-GR Sample Collected in the APG Pilot Plant at the 3D2 Location

\begin{tabular}{|c|c|c|c|c|c|c|}
\hline \multirow{2}{*}{$\begin{array}{l}\text { Spectrum } \\
\text { Number }\end{array}$} & \multirow[b]{2}{*}{ Formula } & \multirow[b]{2}{*}{ MW } & \multirow[b]{2}{*}{ Compound } & \multicolumn{2}{|c|}{ Concentration } & \multirow{2}{*}{$\begin{array}{l}\text { Amount } \\
\text { (ng) }\end{array}$} \\
\hline & & & & (ng/L) & (poby) & \\
\hline $\begin{array}{l}107 \\
107 \\
108 \\
113 \\
118 \\
118 \\
124 \\
126 \\
127 \\
132 \\
134 \\
134 \\
139 \\
144 \\
145 \\
157 \\
160 \\
160 \\
168 \\
177 \\
185 \\
196 \\
197 \\
197 \\
201 \\
201 \\
207 \\
208 \\
217 \\
231 \\
236 \\
240 \\
249 \\
269 \\
276 \\
291 \\
306 \\
319 \\
320 \\
328 \\
322 \\
332 \\
339 \\
345 \\
369 \\
374 \\
\end{array}$ & 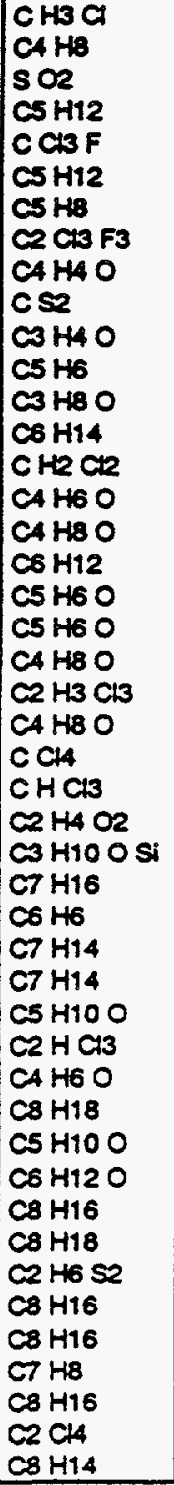 & $\begin{array}{r}50 \\
56 \\
64 \\
72 \\
136 \\
72 \\
68 \\
186 \\
68 \\
76 \\
56 \\
66 \\
58 \\
86 \\
84 \\
70 \\
72 \\
84 \\
82 \\
82 \\
72 \\
132 \\
72 \\
152 \\
118 \\
60 \\
90 \\
100 \\
78 \\
98 \\
98 \\
86 \\
130 \\
70 \\
114 \\
86 \\
100 \\
112 \\
114 \\
94 \\
112 \\
112 \\
92 \\
112 \\
164 \\
110 \\
\end{array}$ & 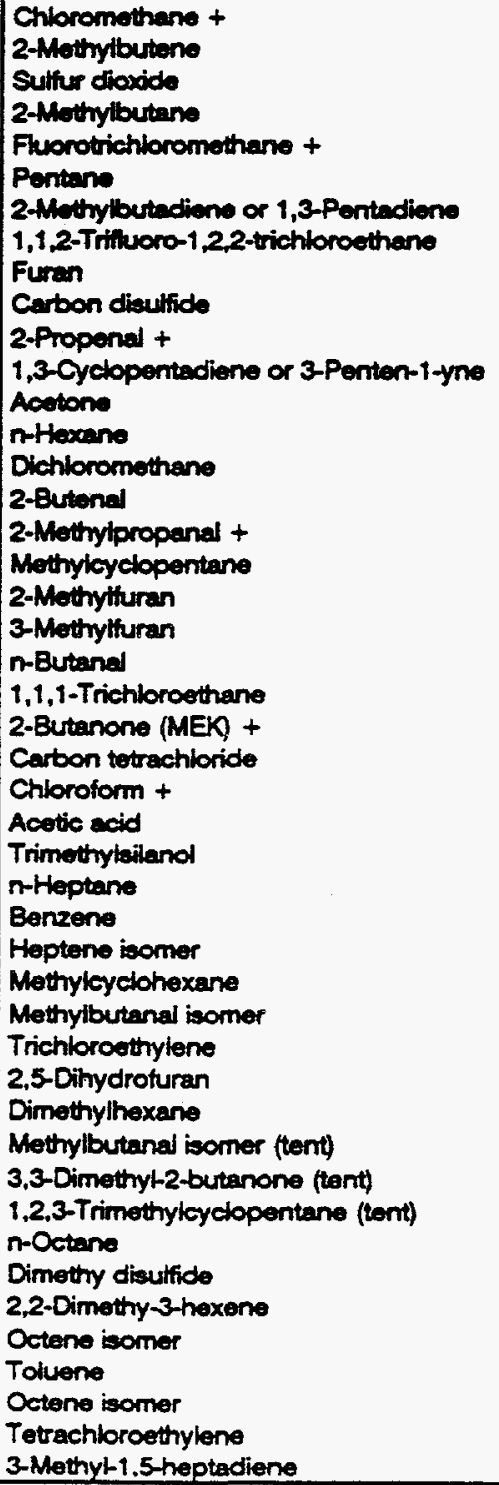 & $\begin{aligned} & 0.4 \\
& 0.3 \\
& 1.1 \\
& 0.4 \\
& \\
& 0.1 \\
& 0.1 \\
& 0.4 \\
& 0.1 \\
& 0.6 \\
& 0.6 \\
& 0.1 \\
&<0.1 \\
&<0.1 \\
&<0.1 \\
& \\
& 0.2 \\
& 0.0 \\
& 0.1 \\
&<0.1 \\
& 0.1 \\
& 0.1 \\
& 0.1 \\
& 0.2 \\
& 0.4 \\
& 0.6 \\
&<0.1 \\
&<0.1 \\
&<0.1 \\
& 0.1 \\
& 0.1 \\
&<0.1 \\
& 0.3 \\
&<0.1 \\
& 0.5 \\
& 0.3 \\
& 0.1 \\
& 0.1 \\
& 0.1 \\
& 2.3 \\
& 0.1 \\
& 0.1 \\
& 0.1\end{aligned}$ & 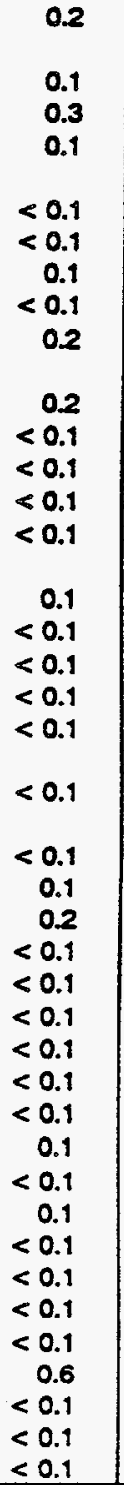 & $\begin{array}{r}7.2 \\
5.1 \\
22 \\
8.1 \\
\\
1.7 \\
1.0 \\
7.0 \\
1.4 \\
12 \\
\\
12 \\
2.7 \\
0.4 \\
0.8 \\
0.4 \\
\\
4.3 \\
0.8 \\
1.5 \\
0.7 \\
2.3 \\
\\
2.6 \\
\\
3.4 \\
8.8 \\
13 \\
\text { trace } \\
\text { trace } \\
0.9 \\
2.6 \\
1.5 \\
\text { trace } \\
5.8 \\
0.8 \\
9.5 \\
5.1 \\
1.7 \\
2.1 \\
1.4 \\
46 \\
2.7 \\
1.4 \\
0.6 \\
\end{array}$ \\
\hline
\end{tabular}

Concentrations are semi-quantitative estimates determined by comparison to the response for a known amount of Toluene. tent = Tentative Identification 
Table 4. Air Monitoring Results Determined from the Tenax-GR Sample Collected in the APG Pilot Plant at the 3D2 Location

Sample Code : TC2210

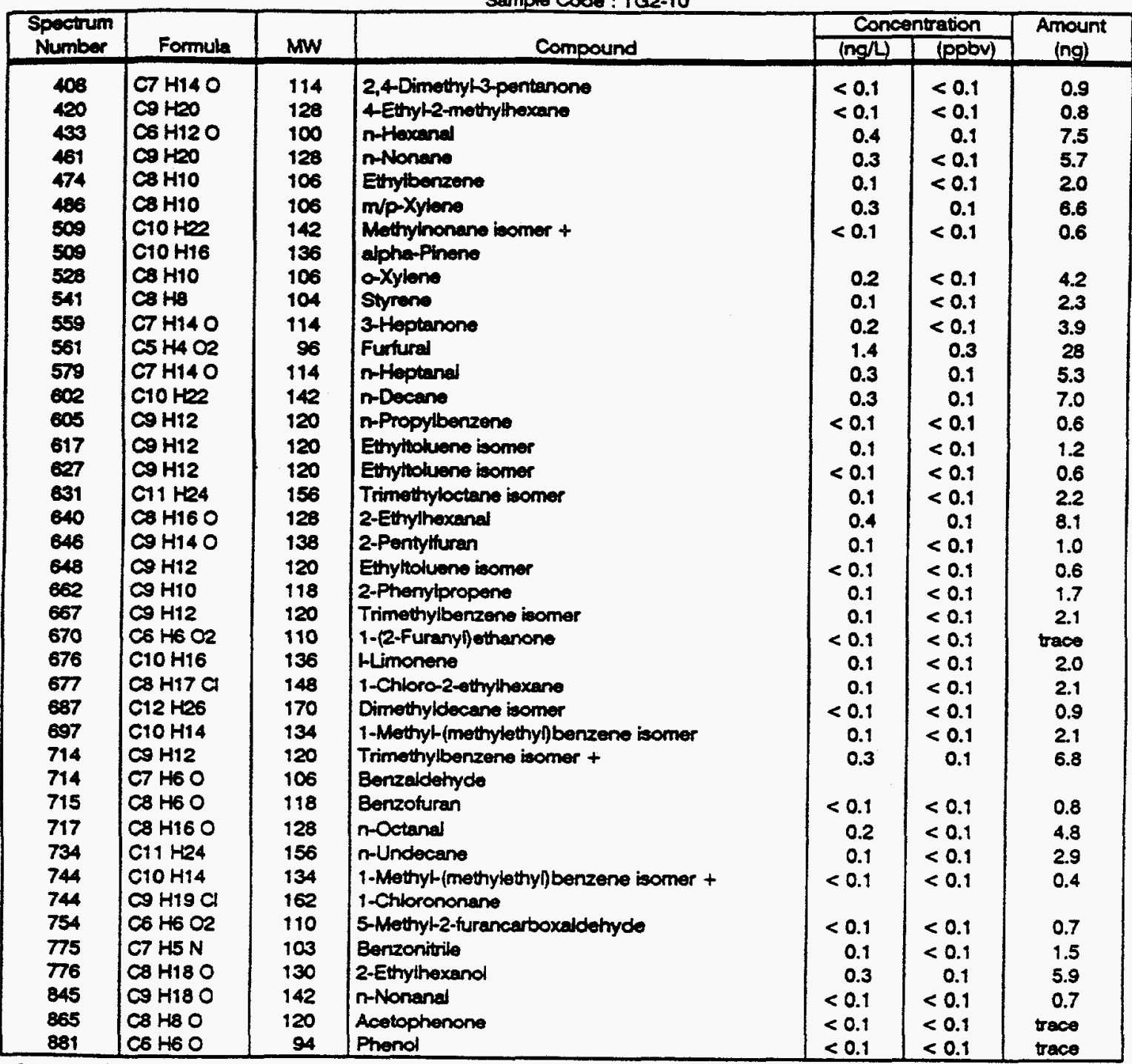

concentrations are semi-quantitative estimates determined by comparison to the response for a known amount of Toluene. tent = Tentative identification 
Tablo 5. Air Monitoring Recults Determined from the Tenex-GR Samplo Collected in the APQ Pilot Plant at the 4D2 Location

\begin{tabular}{|c|c|c|c|c|c|c|}
\hline \multirow{2}{*}{$\begin{array}{l}\text { Spectrum } \\
\text { Number }\end{array}$} & \multirow[b]{2}{*}{ Formula } & \multirow[b]{2}{*}{ MW } & \multirow[b]{2}{*}{ Compound } & \multicolumn{2}{|c|}{ Concentration } & \multirow{2}{*}{$\begin{array}{c}\text { Amount } \\
\text { (ng) }\end{array}$} \\
\hline & & & & (ng/L) & (ppbv) & \\
\hline $\begin{array}{l}90 \\
90 \\
96 \\
96 \\
99 \\
99 \\
108 \\
105 \\
106 \\
108 \\
108 \\
112 \\
113 \\
114 \\
117 \\
118 \\
119 \\
124 \\
124 \\
130 \\
136 \\
136 \\
139 \\
141 \\
141 \\
141 \\
147 \\
156 \\
160 \\
165 \\
165 \\
173 \\
175 \\
175 \\
177 \\
177 \\
181 \\
187 \\
187 \\
197 \\
228 \\
231 \\
238 \\
276 \\
276\end{array}$ & 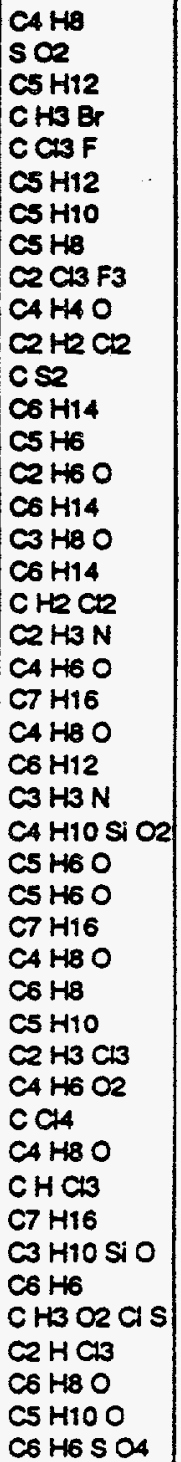 & $\begin{array}{r}56 \\
64 \\
72 \\
94 \\
136 \\
72 \\
70 \\
68 \\
186 \\
68 \\
96 \\
76 \\
86 \\
66 \\
46 \\
86 \\
58 \\
86 \\
84 \\
41 \\
70 \\
100 \\
72 \\
84 \\
53 \\
118 \\
82 \\
82 \\
100 \\
72 \\
80 \\
70 \\
132 \\
86 \\
152 \\
72 \\
118 \\
100 \\
90 \\
78 \\
114 \\
130 \\
96 \\
86 \\
174 \\
\end{array}$ & 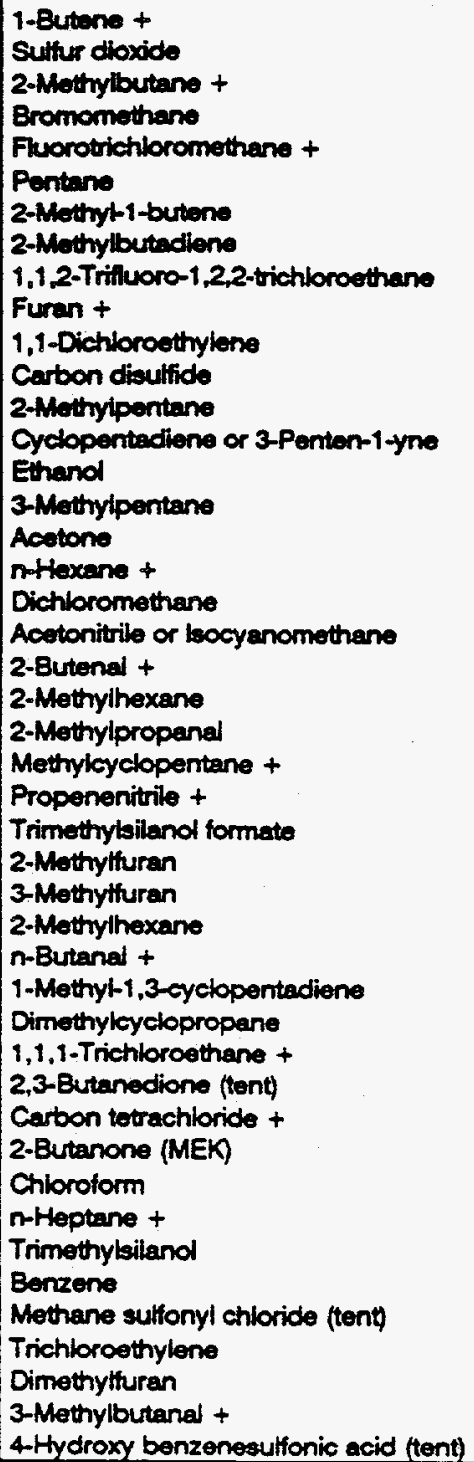 & $\begin{array}{r}0.6 \\
0.1 \\
<0.1 \\
0.1 \\
<0.1 \\
0.3 \\
0.2 \\
<0.1 \\
0.3 \\
\\
0.4 \\
0.1 \\
0.2 \\
<0.1 \\
0.1\end{array}$ & $\begin{array}{r}0.4 \\
1.0 \\
0.1 \\
<0.1 \\
0.1 \\
<0.1 \\
0.1 \\
0.1 \\
0.1 \\
<0.1 \\
0.1 \\
<0.1 \\
1.1 \\
0.1 \\
0.1 \\
0.1 \\
<0.1 \\
<0.1\end{array}$ & $\begin{array}{r}21 \\
67 \\
\text { trace } \\
8.2 \\
1.3 \\
3.8 \\
2.8 \\
8.5 \\
\\
3.9 \\
5.6 \\
1.0 \\
4.9 \\
1.8 \\
59 \\
9.4 \\
\\
2.2 \\
5.0 \\
\text { tace } \\
0.3 \\
1.0\end{array}$ \\
\hline
\end{tabular}

Concentrations are semi-quantitative estimates determined by comparison to the response for a known amount of Toluene. tent = Tentative ldentification 
Table 5. Air Monitoring Reaults Determined trom the Tenax-GR Sample Collected in the APG Pilot Plant at the 4D2 Location

Sample code : TG2-04

\begin{tabular}{|c|c|c|c|c|c|c|}
\hline \multirow{2}{*}{$\begin{array}{l}\text { Spectrum } \\
\text { Number }\end{array}$} & \multirow[b]{2}{*}{ Formula } & \multirow[b]{2}{*}{ MW } & \multirow[b]{2}{*}{ Compound } & \multicolumn{2}{|c|}{ Concentration } & \multirow{2}{*}{$\begin{array}{c}\text { Amount } \\
\text { (ng) }\end{array}$} \\
\hline & & & & $(n g / 4)$ & (ppbv) & \\
\hline $\begin{array}{l}305 \\
307 \\
314 \\
326 \\
352 \\
343 \\
357 \\
424 \\
453 \\
466 \\
466 \\
478 \\
478 \\
521 \\
535 \\
546 \\
557 \\
562 \\
575 \\
597 \\
597 \\
612 \\
627 \\
637 \\
672 \\
693 \\
776 \\
776 \\
786 \\
884 \\
852 \\
\end{array}$ & $\begin{array}{l}C 7 H 12 O \\
C 8 H 18 \\
C 2 H 8 S 2 \\
C 7 H 8 \\
C 8 H 16 \\
C 6 H 12 O \\
C 2 C 14 \\
C 6 H 12 O \\
C 8 H 120 \\
C 8 H 10 \\
C H 2 O 2 \\
C 8 H 10 \\
C 2 H 4 O 2 \\
C 8 H 10 \\
C 8 H 1 \\
C 12 H 26 O \\
C 10 H 22 \\
C 5 H 4 O 2 \\
C 7 H 14 O \\
C 10 H 122 \\
C 9 H 12 \\
C 9 H 12 \\
C 13 H 28 \\
C 8 H 16 O \\
C 10 H 16 \\
C 10 H 14 \\
C 7 H 5 N \\
C 8 H 18 O \\
C 4 H 4 O 2 \\
C 6 H 6 O \\
C 7 H 8 O 2\end{array}$ & $\begin{array}{r}112 \\
114 \\
94 \\
92 \\
112 \\
100 \\
164 \\
100 \\
128 \\
106 \\
46 \\
106 \\
60 \\
106 \\
104 \\
186 \\
142 \\
96 \\
114 \\
142 \\
120 \\
120 \\
184 \\
128 \\
136 \\
134 \\
103 \\
130 \\
84 \\
94 \\
124 \\
\end{array}$ & 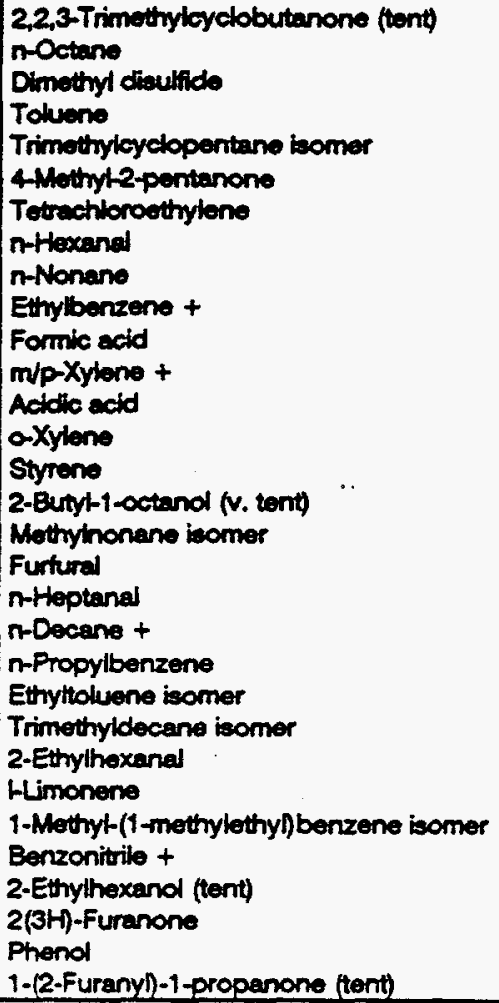 & $\begin{array}{r}0.3 \\
0.1 \\
<0.1 \\
0.5 \\
<0.1 \\
0.1 \\
0.1 \\
0.2 \\
0.2 \\
0.3 \\
\\
1.4 \\
0.3 \\
0.1 \\
0.1 \\
0.1 \\
0.2 \\
0.1 \\
0.3 \\
0.1 \\
0.1 \\
0.1 \\
0.1 \\
<0.1 \\
<0.1 \\
0.2 \\
0.1 \\
0.1 \\
\end{array}$ & $\begin{aligned} & 0.1 \\
&< 0.1 \\
&< 0.1 \\
& 0.1 \\
&<0.1 \\
&<0.1 \\
&<0.1 \\
&<0.1 \\
&<0.1 \\
& 0.1 \\
& \\
& 0.3 \\
& \\
& 0.1 \\
&<0.1 \\
&<0.1 \\
&<0.1 \\
&<0.1 \\
&<0.1 \\
&<0.1 \\
&<0.1 \\
&<0.1 \\
&<0.1 \\
&<0.1 \\
&<0.1 \\
&<0.1 \\
&<0.1 \\
&<0.1 \\
&<0.1 \\
&\end{aligned}$ & $\begin{array}{l}6.3 \\
1.7 \\
1.0 \\
9.3 \\
0.9 \\
2.5 \\
1.0 \\
3.7 \\
4.4 \\
5.3 \\
\\
28 \\
\\
5.0 \\
1.8 \\
1.1 \\
1.3 \\
3.5 \\
2.3 \\
5.2 \\
1.6 \\
1.2 \\
1.3 \\
1.1 \\
0.5 \\
0.7 \\
\\
3.2 \\
1.7 \\
1.1 \\
\end{array}$ \\
\hline
\end{tabular}

Concentrations are semi-quantitative estimates determined by comparison to the response for a known amount of Toluene. tent = Tentative Ldentification

v. tent $=$ Very Tentative Klentification 
Table 6. Air Monitoring Rewults Sorted By Major Compound Clasece Found in the Air Sample Collected at the 113 Location

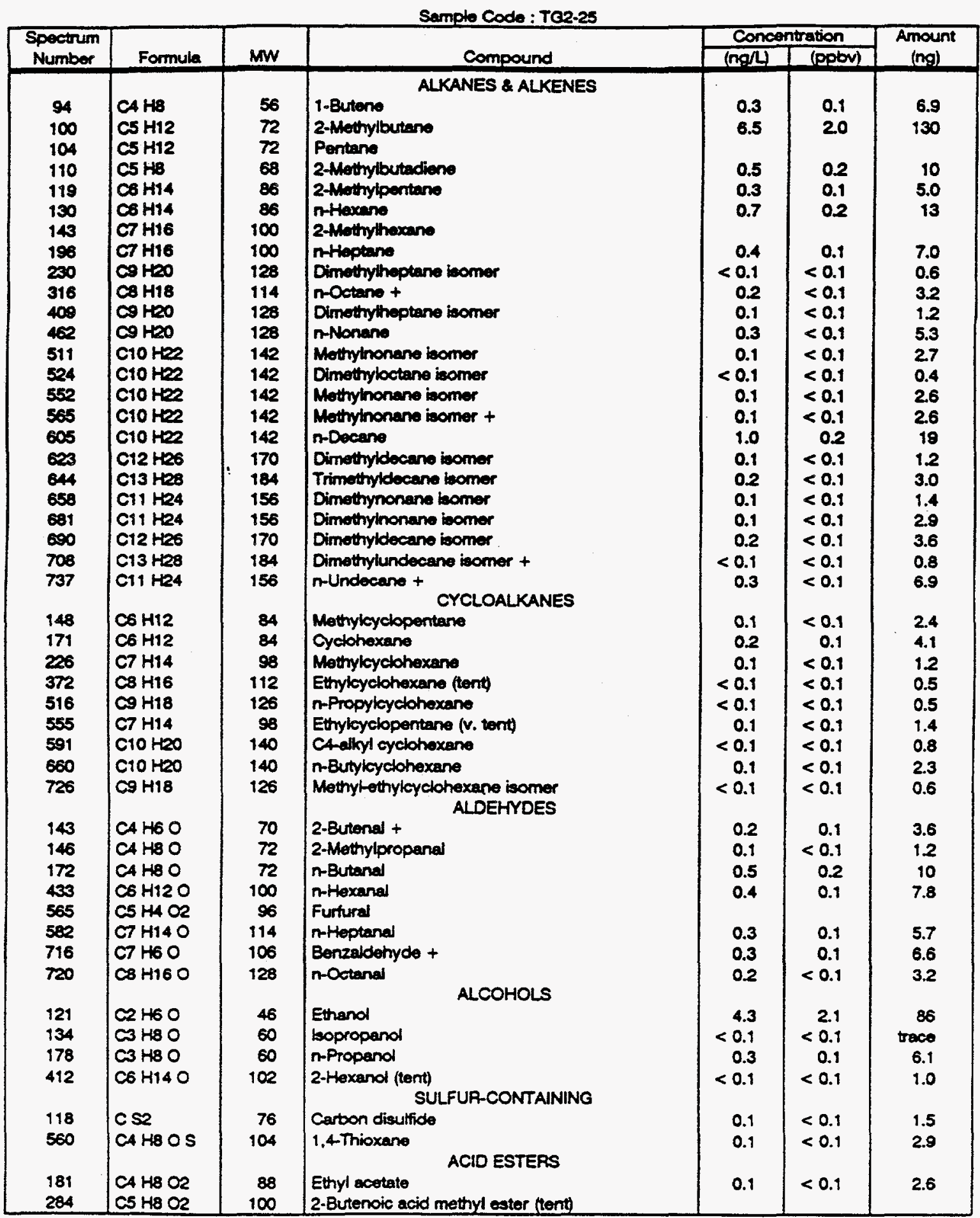

Concentrations are semi-quantitative estimates determined by comparison to the response for a known amount of Toluene. tent = Tentative identification

$v$. tent $=$ Very Tentative Identification 
Table 6. Air Monitoring Results Sorted By Major Compound Classes Found in the Air Sample Collected at the 113 Location

\begin{tabular}{|c|c|c|c|c|c|c|}
\hline \multirow{2}{*}{$\begin{array}{l}\text { Spectrum } \\
\text { Number }\end{array}$} & \multirow[b]{2}{*}{ Formula } & \multirow[b]{2}{*}{ MW } & \multirow[b]{2}{*}{ Compound } & \multicolumn{2}{|c|}{ Concentration } & \multirow{2}{*}{$\begin{array}{l}\text { Arnount } \\
\text { (ng) }\end{array}$} \\
\hline & & & & $\operatorname{lng} / 4)$ & (pabv) & \\
\hline & & & KETONES & & & \\
\hline 125 & $\infty 480$ & 58 & Acetone & 14 & 5.5 & 284 \\
\hline $\begin{array}{l}185 \\
245\end{array}$ & $\begin{array}{l}C 4 H 80 \\
C 5 H 100\end{array}$ & $\begin{array}{l}72 \\
86\end{array}$ & $\begin{array}{l}\text { 2-Butanone (MEK) } \\
\text { 3-Mettul-2 butanone }\end{array}$ & $\begin{array}{l}0.3 \\
0.1\end{array}$ & $\begin{array}{r}0.1 \\
<0.1\end{array}$ & 6.8 \\
\hline 284 & CS H10O & 88 & 2.Pentanone (MPK) + & 0.2 & $<0.1$ & 3.5 \\
\hline 301 & $\mathrm{C} 6 \mathrm{H12O}$ & 100 & 3,3-Dimethyl-2 butanone & $<0.1$ & $<0.1$ & 0.9 \\
\hline 316 & $\mathrm{C7} \mathrm{H12O}$ & 112 & 2,2,3-Trimithyloyclobutanono & & & \\
\hline 352 & C6 H12O & 100 & 4-Mothyl-2-pentanone & $<0.1$ & $<0.1$ & 0.7 \\
\hline 564 & C7 H14 O & 114 & 3-Heptanone + & 0.1 & $<0.1$ & 2.2 \\
\hline 580 & $\mathrm{C7} \mathrm{H} 14 \mathrm{O}$ & 114 & 2-Heptanono & 0.1 & $<0.1$ & 1.2 \\
\hline 618 & 064100 & 98 & Cyclohexanone & 0.1 & $<0.1$ & 2.4 \\
\hline 634 & C8H16O & 128 & $\begin{array}{l}\text { 6-Methyl 3 hoptanone } \\
\text { AROMATICS }\end{array}$ & 0.7 & 0.1 & 14 \\
\hline 205 & C6 H6 & 78 & Benzeno & 0.4 & 0.1 & 7.4 \\
\hline 335 & C7 H8 & 92 & Toluene & 1.1 & 0.3 & 21 \\
\hline 475 & $\mathrm{Cs} \mathrm{H1O}$ & 106 & Etmylbenzene & 0.2 & $<0.1$ & 4.1 \\
\hline 486 & $\mathrm{Cs} \mathrm{H} 10$ & 105 & $\mathrm{~m} / \mathrm{p}$-Xylene & 1.8 & 0.4 & 36 \\
\hline 520 & C8 H1O & 106 & o-xylene & 1.1 & 0.2 & 22 \\
\hline 543 & $\mathrm{CoH} H$ & 104 & Styrene & 0.3 & 0.1 & 6.2 \\
\hline 564 & $\mathrm{COH12}$ & 120 & teopropytbenzene & & & \\
\hline 607 & $\infty \mathrm{H} 12$ & 120 & n-Propylbenzene & 0.1 & $<0.1$ & 1.5 \\
\hline$\infty 20$ & $\infty \mathrm{H12}$ & 120 & Ethyttoluene isomer & 0.3 & 0.1 & 5.7 \\
\hline $6 \infty$ & $\infty \mathrm{Hi2}$ & 120 & Trimethylbenzene isomer & 0.4 & 0.1 & 7.6 \\
\hline 651 & $\mathrm{CH} \mathrm{H12}$ & 120 & Ethyltoluene isomer & 0.2 & $<0.1$ & 3.9 \\
\hline 668 & $\mathrm{COH}+2$ & 120 & Trimethylbenzene isomer & 0.9 & 0.2 & 18 \\
\hline 700 & $\mathrm{CtOH}$ & 134 & 1- Methyl-(1-methylethyl) benzene isomer & 0.2 & $<0.1$ & 3.5 \\
\hline 702 & $\mathrm{C} 10 \mathrm{H} 18$ & 138 & Decahydronaphthalene & $<0.1$ & $<0.1$ & 0.7 \\
\hline 715 & $\mathrm{Cg} H 12$ & 120 & Trimethylbenzene isomer & 0.3 & 0.1 & 5.6 \\
\hline 734 & $\infty \mathrm{H}_{10}$ & 118 & Propenylbenzene & $<0.1$ & $<0.1$ & 0.7 \\
\hline 737 & $\mathrm{C} 10 \mathrm{H} 14$ & 134 & Methylpropylbenzene & & & \\
\hline 746 & $\mathrm{ClOH}_{4}$ & 134 & Dimethylethylbenzene isomer & 0.1 & $<0.1$ & 1.9 \\
\hline 764 & $\mathrm{C1OH} 44$ & 134 & $\begin{array}{l}\text { sec-Butylbenzene } \\
\text { HALOGEN-CONTAINING }\end{array}$ & $<0.1$ & $<0.1$ & 0.5 \\
\hline 104 & $\cos F$ & 136 & Fluorotrichloromethane + & 9.1 & 1.5 & 182 \\
\hline 112 & $C_{2} C_{3} F_{3}$ & 186 & 1,1,2-Trifluoro-1,2,2-trichloroothane & 0.3 & $<0.1$ & 5.5 \\
\hline 131 & $\mathrm{CH}_{2} \mathrm{Cl}_{2}$ & 84 & Dichibromethano & 0.3 & 0.1 & 6.4 \\
\hline 183 & $\mathrm{C}_{2} \mathrm{H}_{3} \mathrm{Cl}_{3}$ & 132 & 1,1,1-Trichloroethane & 0.2 & $<0.1$ & 4.5 \\
\hline 180 & $\mathrm{CHCH}$ & 118 & Chioroform & $<0.1$ & $<0.1$ & 0.8 \\
\hline 240 & $\mathrm{C} 2 \mathrm{HCl} 3$ & 130 & Trichioroethylene & 0.2 & $<0.1$ & 3.5 \\
\hline 366 & $\mathrm{ClCH}_{2}$ & 164 & $\begin{array}{l}\text { Tetrechloroethylene } \\
\text { NITROGEN-CONTAINING }\end{array}$ & 0.2 & $<0.1$ & 4.2 \\
\hline 141 & $\mathrm{C} 2 \mathrm{HBN}$ & 41 & Acotonitrile or bocyanomethane & 0.2 & 0.1 & 4.5 \\
\hline 170 & $\mathrm{CAH}$ H NO & 85 & 2-bocyanatopropane (tent) & $<0.1$ & $<0.1$ & 1.1 \\
\hline 685 & C7 HONO & 123 & 2-Methoxybenzenamine & 0.1 & $<0.1$ & 1.3 \\
\hline 708 & $\mathrm{Cs} H 13 \mathrm{~N}$ & 123 & 1-Butyl-1H-pyrrole & & & \\
\hline$m$ & C7 H5 N & 103 & $\begin{array}{l}\text { Benzonitrile } \\
\text { OTHER OXYGEN-CONTAINING }\end{array}$ & 0.1 & $<0.1$ & 2.3 \\
\hline 113 & $\mathrm{CAHAO}$ & 68 & Furan & 0.5 & 0.1 & 9.1 \\
\hline 154 & C5 H6O & 82 & 2-Methylfuran & 0.4 & 0.1 & 8.7 \\
\hline 164 & C5 H6O & 82 & 3-Methythuran & $<0.1$ & $<0.1$ & 0.6 \\
\hline 294 & C7 H1O O & 110 & 2-Propytturan & $<0.1$ & $<0.1$ & 0.5 \\
\hline 716 & $\cos 460$ & 198 & Benzoturan (tent) & & & \\
\hline & & & TERPENES & & & \\
\hline 559 & C10 H16 & 136 & Cemphene & $<0.1$ & $<0.1$ & 0.7 \\
\hline 678 & $\mathrm{C}_{10 \mathrm{H} 16}$ & 136 & Limonene & 0.1 & $<0.1$ & 3.0 \\
\hline
\end{tabular}

Concentrations are semi-quantitative estimates determined by comparison to the response for a known amount of Toluene. tent = Tentative Identification 
Table 7. Ar Monitoring Results Sorted By Major Compound Classes Found in the Air Sample Collected at the 2D2 Location

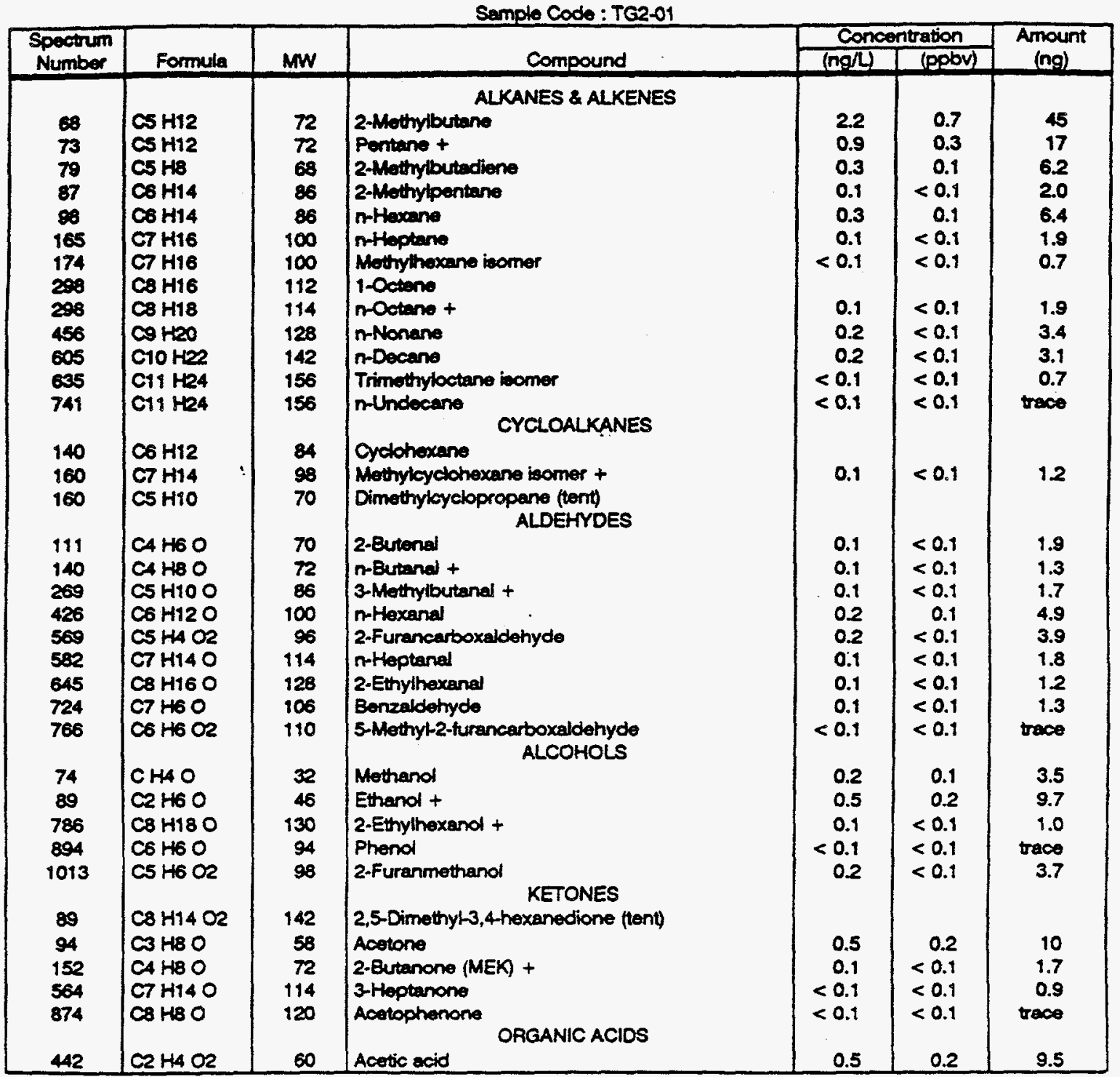

Concentrations are semi-quantitative estimates determined by comparison to the response for a known amount of Toluene. tert $=$ Tentative identification 
Table 7. Air Monitoring Results Sorted By Major Compound Clasees Found in the Air Sample Collected at the 2D2 Location

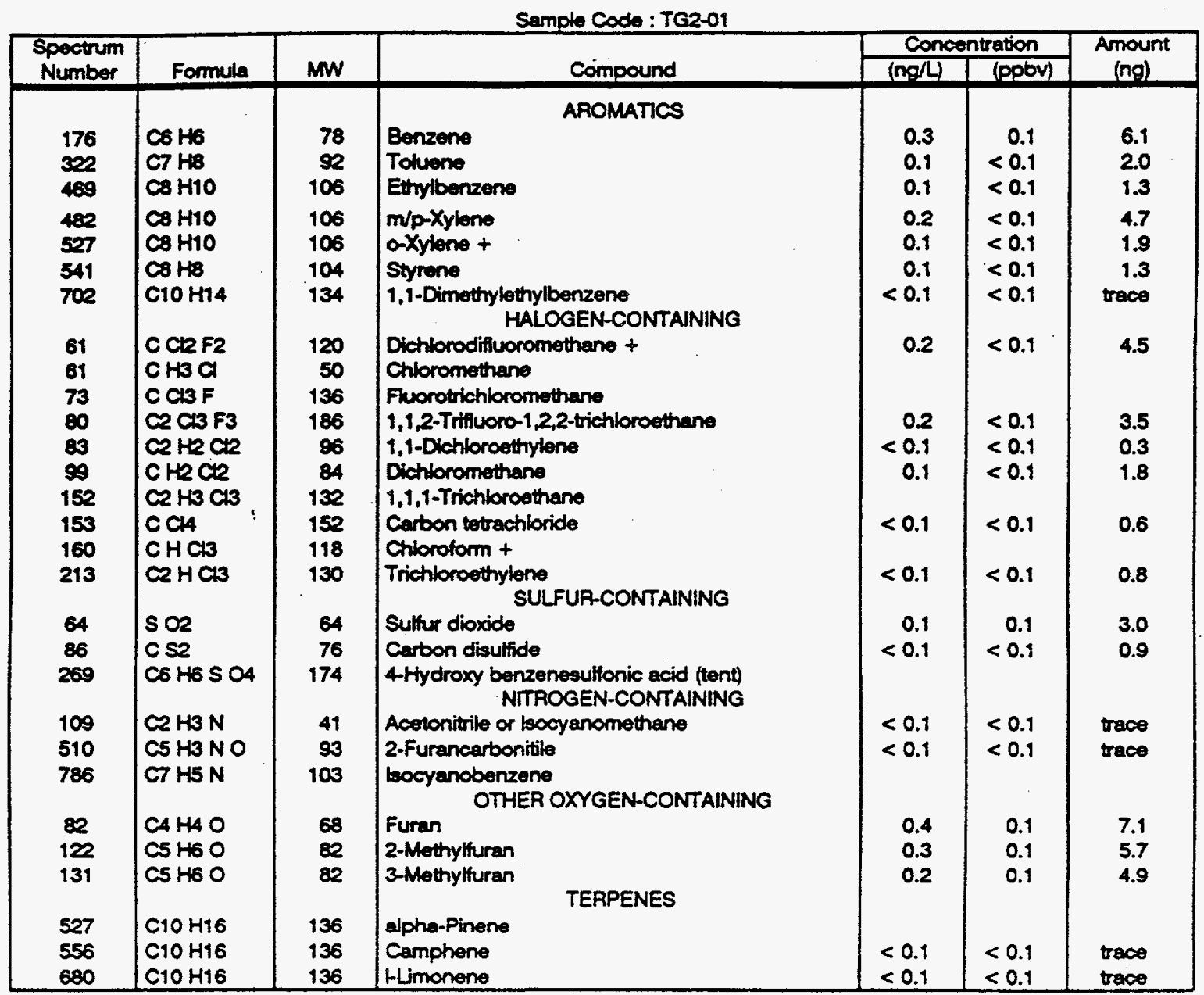

Concentrations are seminquantitative stimates determined by comparison to the response for a known amount of Toluene. tent = Tentative Identification 
Table 8. Air Moniloring Results Sorted By Mejor Compound Clesses Found in the Air Sample Collected at the 3D2 Location

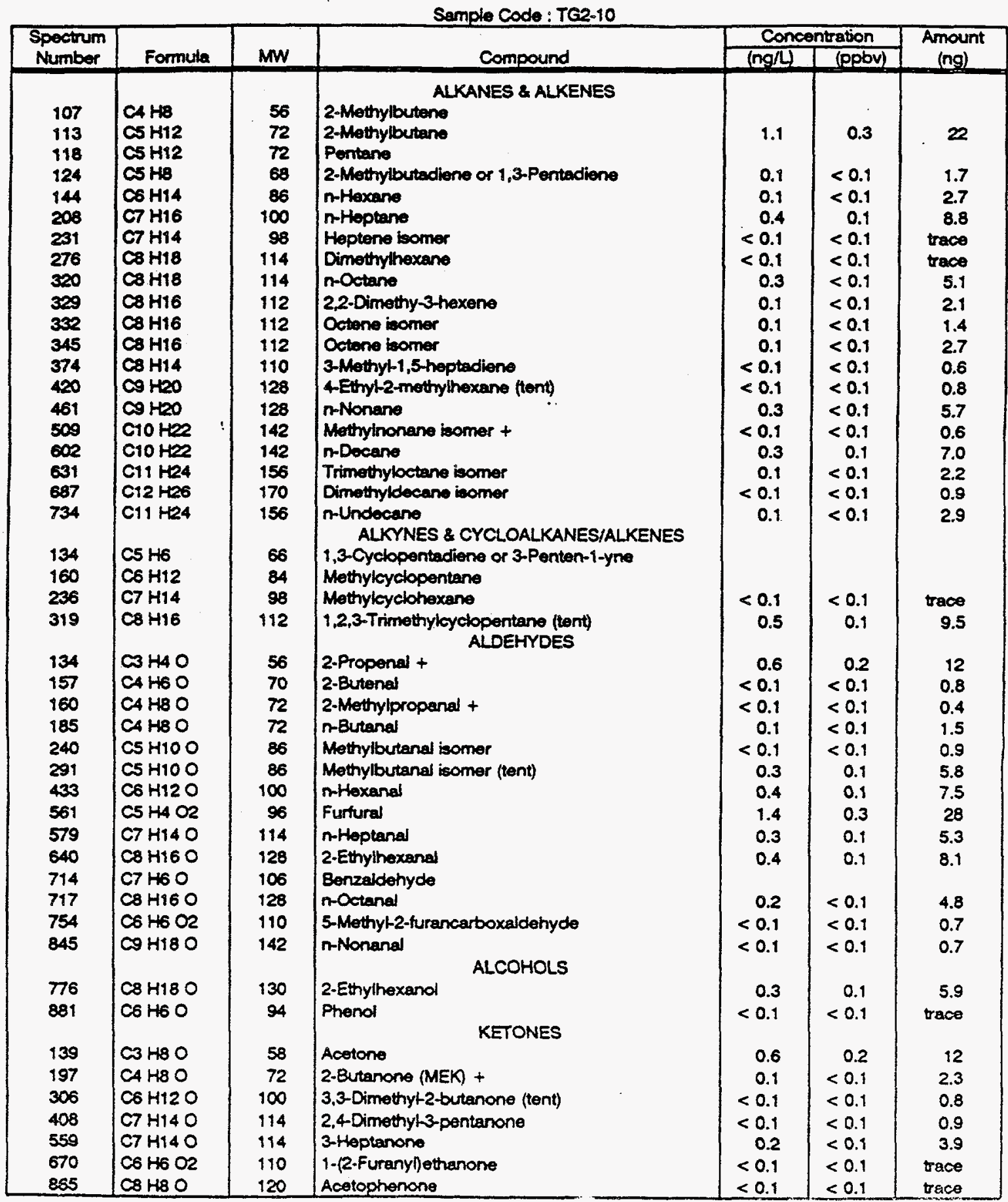

Concsntrations are semi-quantitetive ostimates determiniod by comparis un to the responise for a known ernount of Toluene. tent = Tentative identification 
Table 8. Air Monitoring Results Sorted By Major Compound Classes Found in the Air Sample Collected at the 3D2 Location

\begin{tabular}{|c|c|c|c|c|c|c|}
\hline \multirow{2}{*}{$\begin{array}{l}\text { Spectrum } \\
\text { Number }\end{array}$} & \multirow[b]{2}{*}{ Formula } & \multirow[b]{2}{*}{$M W$} & \multirow[b]{2}{*}{ Compound } & \multicolumn{2}{|c|}{ Concentration } & \multirow{2}{*}{$\begin{array}{l}\text { Amount } \\
\text { (ng) }\end{array}$} \\
\hline & & & & $(n g / L)$ & (ppbv) & \\
\hline 201 & $\mathrm{C} 2 \mathrm{H} 402$ & 60 & $\begin{array}{l}\text { ORGANIC ACIDS } \\
\text { Acetic acid } \\
\text { AROMATICS }\end{array}$ & & & \\
\hline $\begin{array}{l}217 \\
339 \\
474\end{array}$ & $\begin{array}{l}\text { C6 H6 } \\
\text { C7 HB } \\
\text { C8 H10 }\end{array}$ & $\begin{array}{r}78 \\
92 \\
106\end{array}$ & $\begin{array}{l}\text { Berzzene } \\
\text { Tolvione } \\
\text { Ethylbenzene }\end{array}$ & $\begin{array}{l}0.6 \\
2.3 \\
0.1\end{array}$ & $\begin{array}{r}0.2 \\
0.6 \\
<0.1\end{array}$ & $\begin{array}{r}13 \\
46 \\
2.0\end{array}$ \\
\hline 486 & C8 H10 & 106 & mp-Xylene & 0.3 & 0.1 & 6.6 \\
\hline 528 & $\mathrm{CoH} \mathrm{H}_{10}$ & 105 & oxylene & 0.2 & $<0.1$ & 4.2 \\
\hline 541 & $\mathrm{COHB}$ & 104 & Styrene & 0.1 & $<0.1$ & 2.3 \\
\hline 605 & $\mathrm{COH}$ & 120 & n-Propybenzene & $<0.1$ & $<0.1$ & 0.6 \\
\hline 617 & $\infty \mathrm{H} 12$ & 120 & Ethyltoluene isomer & 0.1 & $<0.1$ & 1.2 \\
\hline 627 & $\infty \mathrm{H}_{12}$ & 120 & Ethyltoluene isomer & $<0.1$ & $<0.1$ & 0.6 \\
\hline 648 & $\infty \mathrm{H} 12$ & 120 & Ethyttoluene isomer & $<0.1$ & $<0.1$ & 0.6 \\
\hline 620 & $\infty \mathrm{H} 1 \mathrm{O}$ & 118 & 2.Phenylpropene & 0.1 & $<0.1$ & 1.7 \\
\hline 667 & $\infty \mathrm{H}_{12}$ & 120 & Trimethylbenzene iomor & 0.1 & $<0.1$ & 2.1 \\
\hline $\begin{array}{l}697 \\
714\end{array}$ & $\begin{array}{l}\mathrm{C10H14} \\
\mathrm{\infty} \mathrm{H12}:\end{array}$ & $\begin{array}{l}134 \\
120\end{array}$ & $\begin{array}{l}\text { 1-Methyt-(methylethyl)benzene bomer } \\
\text { Trimethylbenzene isomer + }\end{array}$ & $\begin{array}{l}0.1 \\
0.3\end{array}$ & $\begin{array}{r}<0.1 \\
0.1\end{array}$ & $\begin{array}{l}2.1 \\
6.8\end{array}$ \\
\hline $\begin{array}{l}715 \\
744\end{array}$ & $\begin{array}{l}\text { C8 H6O } \\
\text { C10 H14 }\end{array}$ & $\begin{array}{l}118 \\
134\end{array}$ & $\begin{array}{l}\text { Benzohuran } \\
\text { 1-Methyt-(methylethyl)benzene isomer + } \\
\text { HALOGEN-CONTAINING }\end{array}$ & $\begin{array}{l}<0.1 \\
<0.1\end{array}$ & $\begin{array}{l}<0.1 \\
<0.1\end{array}$ & $\begin{array}{l}0.8 \\
0.4\end{array}$ \\
\hline $\begin{array}{l}107 \\
118\end{array}$ & $\begin{array}{l}C+3 \mathrm{Ca} \\
\mathrm{CCA} F\end{array}$ & $\begin{array}{r}50 \\
136\end{array}$ & $\begin{array}{l}\text { Chioromethane + } \\
\text { Fluorotrichloromethane + }\end{array}$ & $\begin{array}{l}0.4 \\
0.4\end{array}$ & $\begin{array}{l}0.2 \\
0.1\end{array}$ & $\begin{array}{l}7.2 \\
8.1\end{array}$ \\
\hline 126 & $\mathrm{C}_{2} \mathrm{Cl3} \mathrm{F3}$ & 186 & 1,1,2-Trifluoro-1,2,2-trichloroethane & 0.1 & $<0.1$ & 1.0 \\
\hline 145 & $\mathrm{CH} \mathrm{H}_{2}$ & 84 & Dichioromethane & $<0.1$ & $<0.1$ & 0.4 \\
\hline 196 & $\mathrm{C2} \mathrm{H}_{3} \mathrm{Cl} 3$ & 132 & 1,1,1-Trichloroethane & $<0.1$ & $<0.1$ & 0.7 \\
\hline 197 & $\mathrm{CCH}$ & 152 & Carbon tetrachloride & & & \\
\hline 201 & $\mathrm{CHCl3}$ & 118 & Chlorolom + & 0.1 & $<0.1$ & 2.6 \\
\hline 249 & $\mathrm{C} 2 \mathrm{HCH}$ & 130 & Trichloroethylene & 0.1 & $<0.1$ & 2.6 \\
\hline 369 & $\mathrm{C2} \mathrm{CH}_{4}$ & 164 & Tetrachloroethylene & 0.1 & $<0.1$ & 1.4 \\
\hline 677 & $\mathrm{Cs} \mathrm{H17} \mathrm{C}$ & 148 & 1-Chloro-2-ethylhexane & 0.1 & $<0.1$ & 2.1 \\
\hline 744 & $C 9 \mathrm{H} 19 \mathrm{Cl}$ & 162 & $\begin{array}{l}\text { 1-Chlorononane } \\
\text { SULFUR-CONTAINING }\end{array}$ & & & \\
\hline 108 & 502 & 64 & Sulfur dioxide & 0.3 & 0.1 & 5.1 \\
\hline 132 & $c s 2$ & 76 & Carbon disutfide & 0.1 & $<0.1$ & 1.4 \\
\hline 328 & $\mathrm{C} 2 \mathrm{H6} \mathrm{S2}$ & 94 & $\begin{array}{l}\text { Dimethy disulfide } \\
\text { NITROGEN-CONTAINING }\end{array}$ & 0.1 & $<0.1$ & 1.7 \\
\hline 775 & $\mathrm{C} 7 \mathrm{H} 5 \mathrm{~N}$ & 103 & $\begin{array}{l}\text { Benzonitrile } \\
\text { OTHER OXYGEN-CONTAINING }\end{array}$ & 0.1 & $<0.1$ & 1.5 \\
\hline 127 & C4 H4O & 68 & Furan & 0.4 & 0.1 & 7.0 \\
\hline 168 & CS H6O & 82 & 2-Mothylfuran & 0.2 & 0.1 & 4.3 \\
\hline 177 & $\cos 160$ & 82 & 3-Methyifuran & 0.0 & $<0.1$ & 0.8 \\
\hline 207 & $\mathrm{C} 3 \mathrm{H} 10 \mathrm{OSi}$ & 90 & Trimethylsiland & 0.2 & $<0.1$ & 3.4 \\
\hline 269 & $\mathrm{CA} H 6 \mathrm{O}$ & 70 & 2,5-Dihydroturan & 0.1 & $<0.1$ & 1.5 \\
\hline 646 & $\mathrm{COH} \mathrm{H}_{14 \mathrm{O}}$ & 138 & 2-Pentyturan & 0.1 & $<0.1$ & 1.0 \\
\hline $\begin{array}{l}509 \\
676\end{array}$ & $\mathrm{C} 10 \mathrm{H} 16$ & 136 & alpha-Pinene & & & \\
\hline 676 & $\mathrm{C} 10 \mathrm{H} 16$ & 136 & HLimonene & 0.1 & $<0.1$ & 2.0 \\
\hline
\end{tabular}

Concentrations are semi-quantitative estimates determined by comparison to the response for a known amouni of Toluene. tent = Tentative Identification 
Table 9. Air Monitoring Rosults Sortod by Major Compound Clawes Found in the Air Sample Collocted at the 402 Location

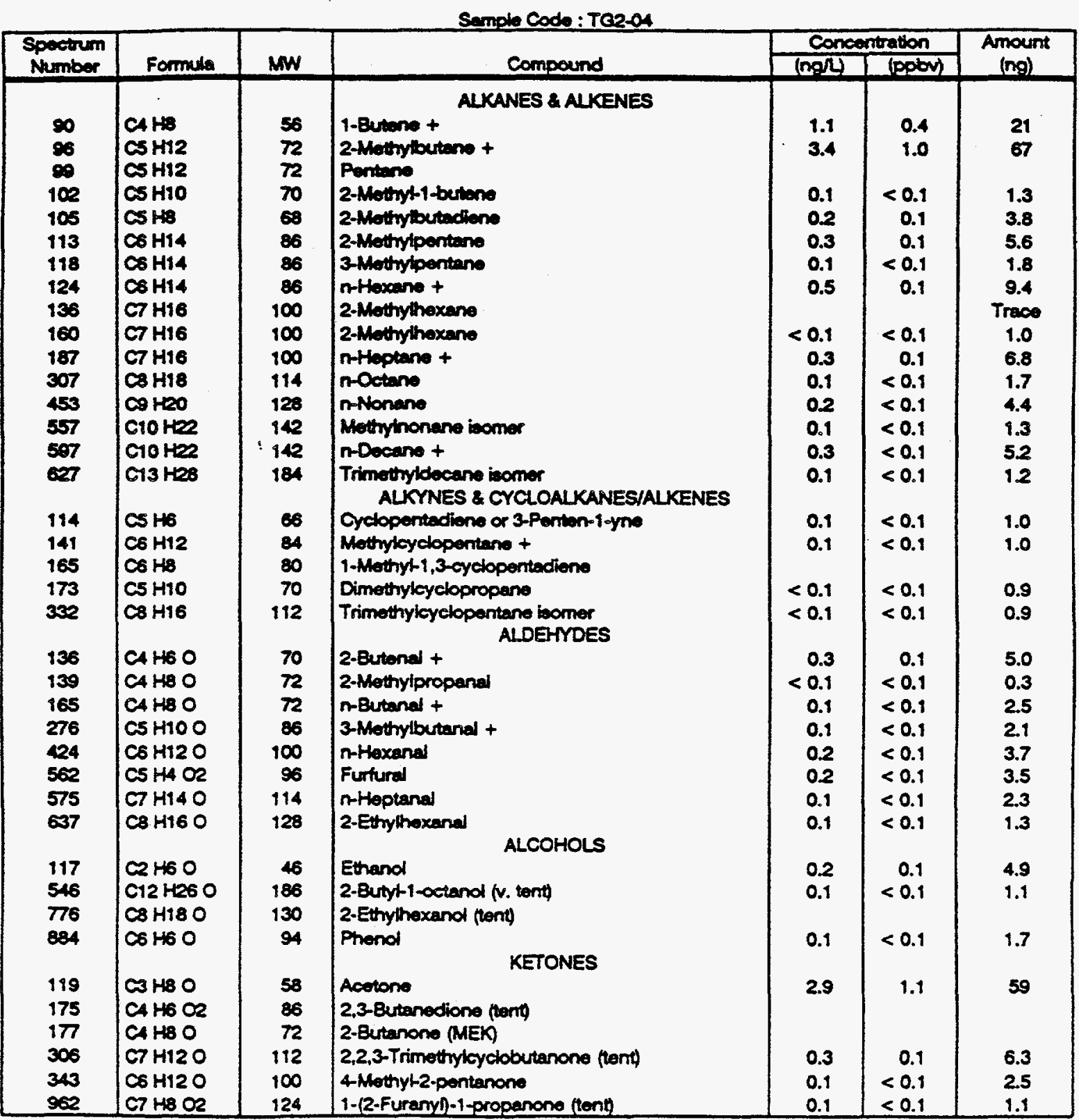

Concentrations are semi-quantitative estimates determined by comparison to the response for a known amount of Toluene. tert = Tentative Identification

$v$. tent = Very Tentative Identification 
Table 9. Air Monitoring Results Sortod by Major Compound Classes Found in the Air Sample Colloctod at the 4D2 Location

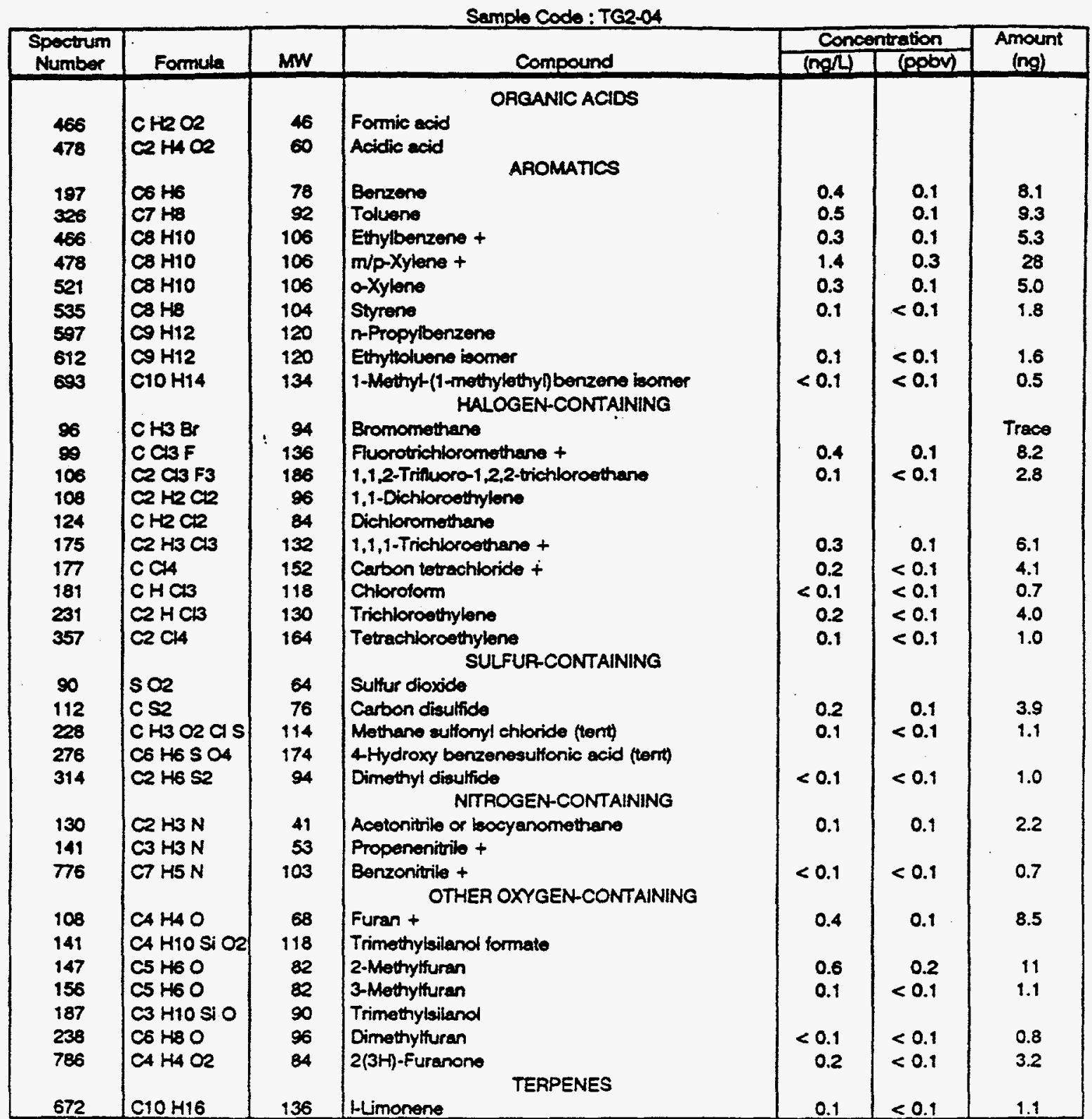

Concentrations are semi-quantitative estimales determined by comparison to the response for a known amount of Toluene. tent = Tentative identification 


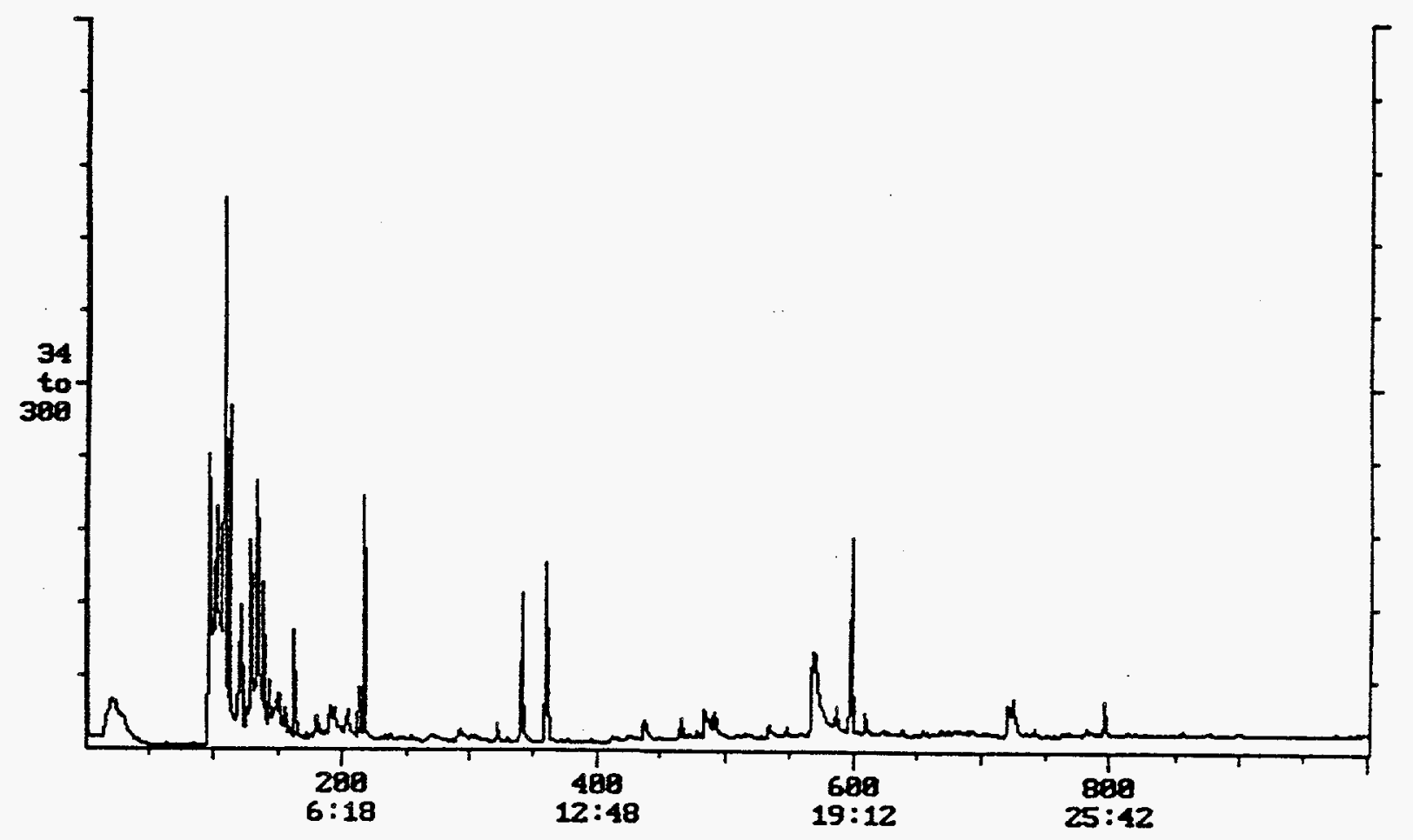

Figure 5. Total Ion Current Profile From the GC/MS Analysis of the Field Blank. 
Tablo 10. Air Monitoring Result Determined trom the Tonax-GR Fiold Blank

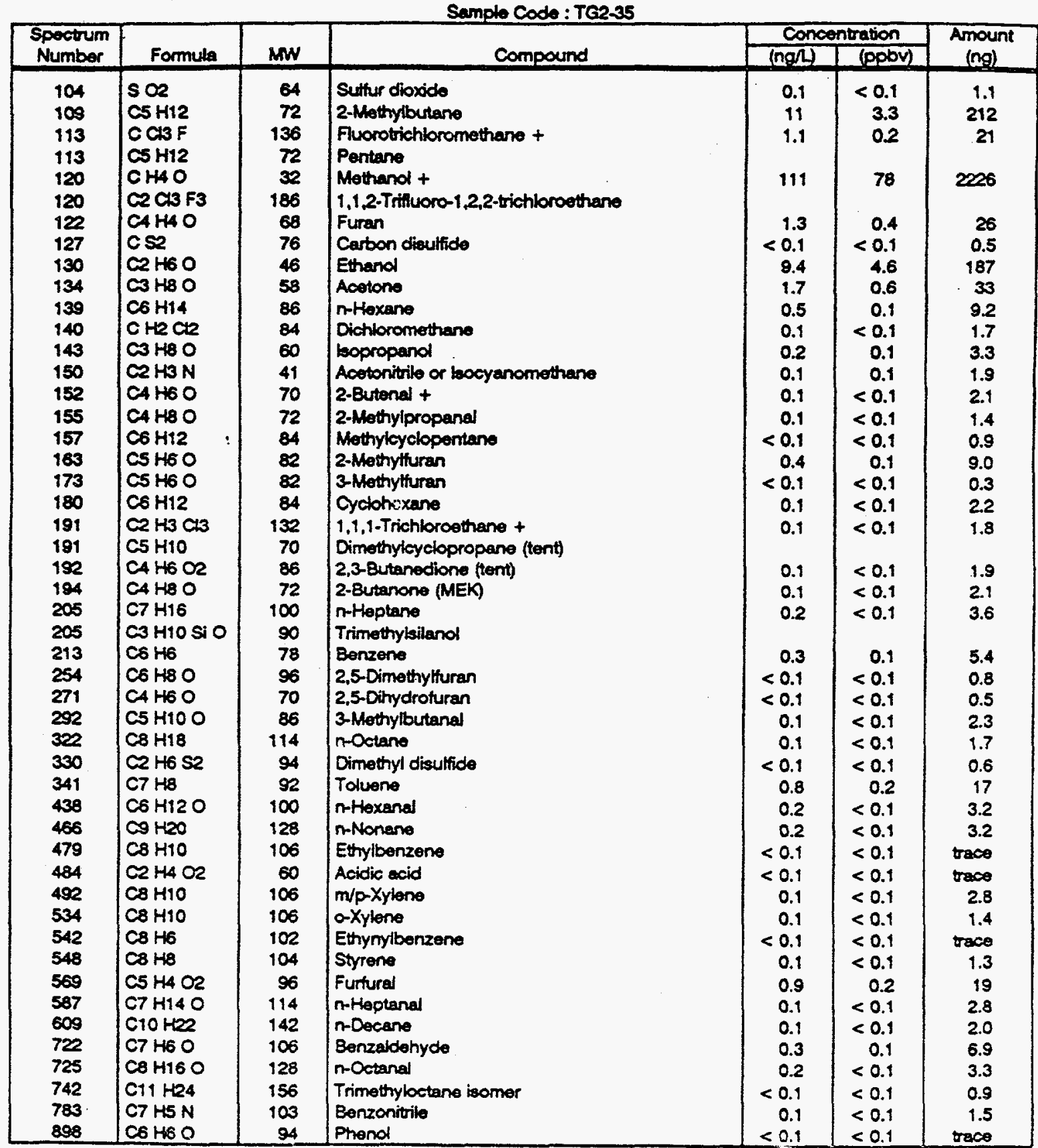

Concentrations are semi-quantitative estimates determined by comparison to the response for a known arnount of Toluene. tent = Tentaive identification 
50

Table 11. Air Monitoring Results Sorted By Major Compound Claseses Found in the Tonax-OR Fleld Blank

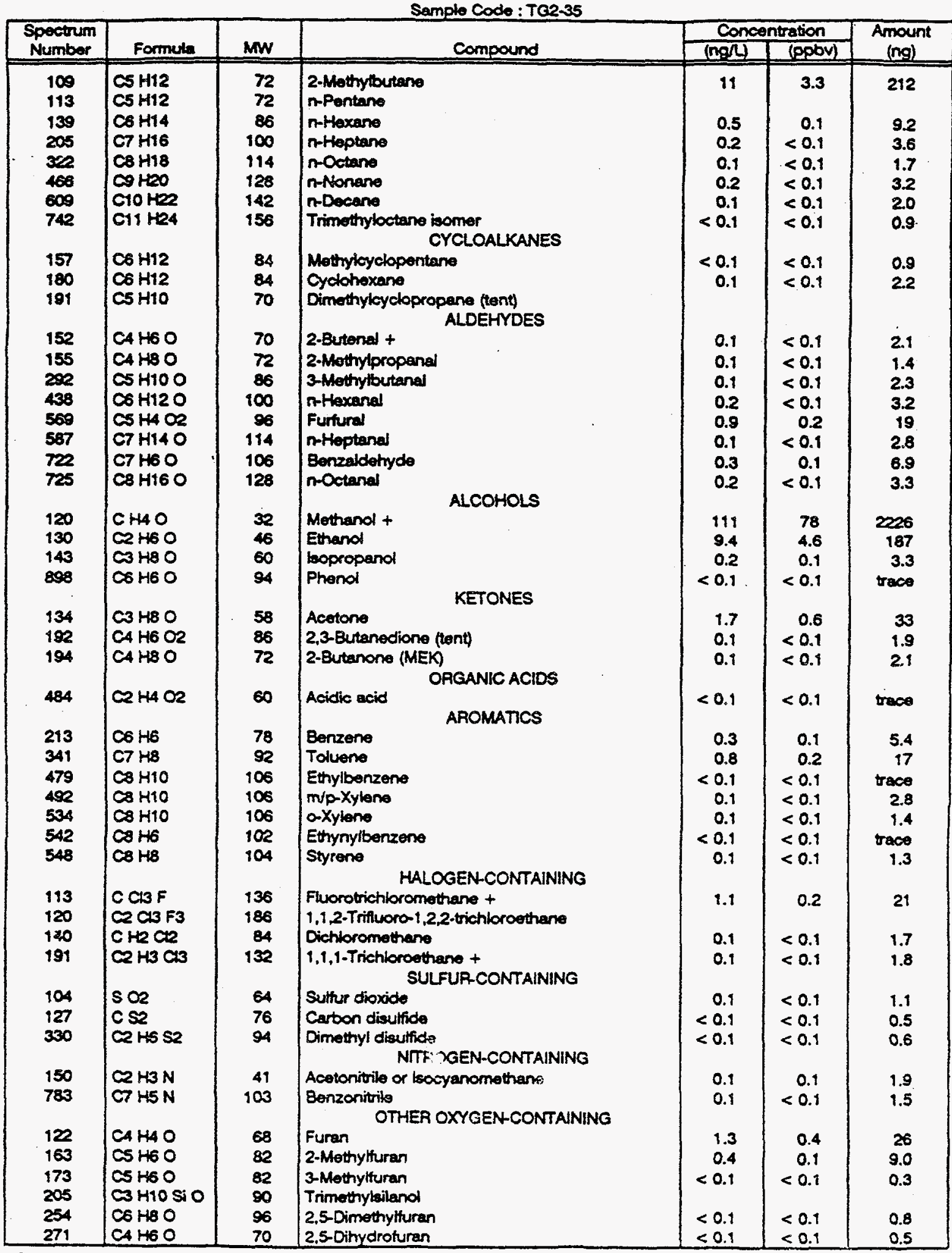

Concontrations are semi-quantitative estimates determined by comparison to the response for a known amount of "Toluone. tort = Tontative Identification 
The VOC levels were in the range where only the 20 liter samples required analyses. The 113 location contained the largest number of identified compounds and the 2D2 location the fewest number. Table 12 provides a summary of the total semi-qua titative results from the four sampled locations.

Table 12. Semiquantitative Result Totals From the Air Monitoring Within the APG Pilot Plant

\begin{tabular}{|c|c|c|c|}
\hline \multirow{2}{*}{ Location } & \multicolumn{2}{|c|}{ Concentration } & \multirow{2}{*}{$\begin{array}{c}\text { Amount } \\
\text { (ng) }\end{array}$} \\
\cline { 2 - 3 } & (ng/L) & (ppbv) & 1085 \\
\hline 113 & 54 & 15 & 198 \\
\hline 2D2 & 9.7 & 2.3 & 327 \\
\hline 3D2 & 16 & 3.1 & 354 \\
\hline 4D2 & 17 & 4.4 & \\
\hline
\end{tabular}




\title{
Appendix B:
}

SciTech Services, Inc., Report on Analysis of Pilot Plant Complex

\author{
Air Samples
}




\section{ANALYSIS OF PILOT PLANT AIR SAMPLES}

February 24, 1995

Prepared for:

Argonne National Laboratory

P.O. Box 96

Aberdeen Proving Ground, Maryland 21010

Prepared by:

SciTech Services, Inc.

1311 Continental Drive, Suite G

Abingdon, Maryland 21009 
TABLE OF CONTENTS

Title

Page

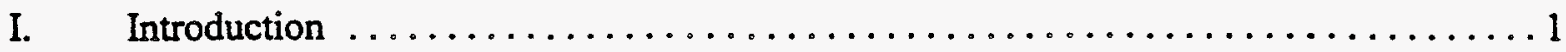

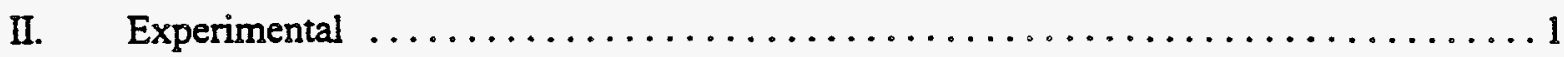

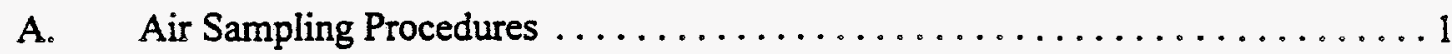

B. Gas Chromatography-Flame Photometric Detector (GC-FPD) Screening

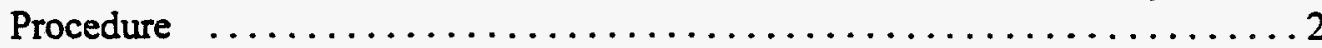

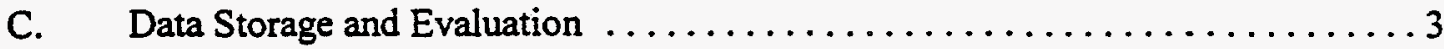

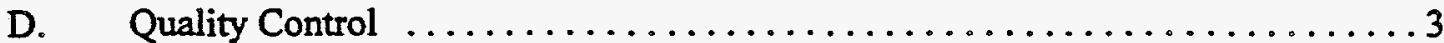

E. CW Agent Confirmation and Analysis for Semivolatiles and Volatiles by

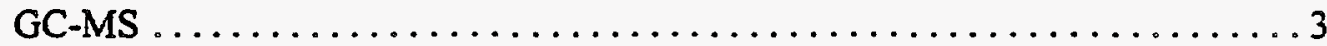

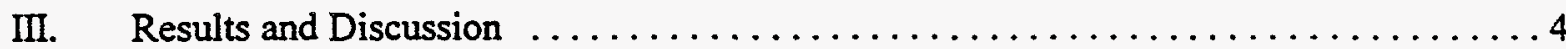

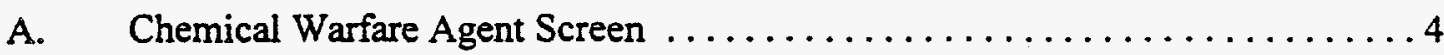

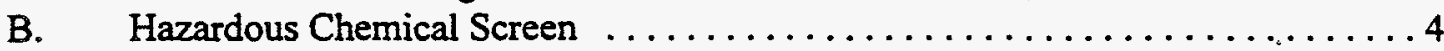

Appendix 1: Figures 1 Through 26

Appendix 2: Tables 2 Through 14

Enclosure 1: Master List of Potential Contaminants of Pilot Plant 


\section{ANALYSIS OF PILOT PLANT AIR SAMPLES}

\section{Introduction}

In support of Argonne National Laboratory's effort to dismantle Building E5625 (the Pilot Plant), SciTech Services, Inc. was asked to analyze a series of air samples collected on Depot Area Agent Monitoring System (DAAMS) tubes containing Tenax sorbent. SciTech's analysis of the Pilot Plant air samples used protocols and methods based on the approved laboratory operating procedures (LOP) of the U.S. Army Edgewood Research, Development and Engineering Center's (ERDEC's) Air Monitoring Branch or standard Environmental Protection Agency (EPA) methods. An extensive list of possible contaminants was composed by Argonne National Laboratory containing over 150 compounds (Enclosure 1). Some are inorganic chemicals or salts of organic chemicals that would not be volatile enough to be detected by DAAMS tube technology, such as lead from paint or sodium hydroxide. Some are volatile solvents commonly found in many chemical facilities. The most volatile components of the list, such as dimethyl ether and chloromethane, should have evaporated and disappeared years ago. The compounds of interest for this air monitoring exercise were split into chemical warfare (CW) agents and non-agents that were volatile or semivolatile. The $C W$ agents were screened by Gas Chromatography - Dual Flame Photometric Detector (GC-(dual) FPD). Nonagent analytes were analyzed by Gas Chromatography-Mass Spectrometry (GC-MS) and included the following categories of compounds: experimental agents, agent precursors, agent breakdown products, agent stimulants, polychlorinated biphenyls (PCBs), polyaromatic hydrocarbons, and halogenated hydrocarbons.

The GC-MS analysis for non-agents used a semiquantitative approach recommended by Argonne based on the detector response to $1 \mathrm{ng}$ of toluene. This response factor was used for the other analytes' selected target ions to generate very approximate concentrations.

Quality control was practiced on several levels. Standard quality control procedures were defined in the Air Monitoring Branch Quality Assurance/Quality Control manual which is available on request. Several steps were taken above the standard procedures. This included steps such as analyzing sorbent tube blanks by GC-MS for laboratory contamination and holding the samples at $0-5^{\circ} \mathrm{C}$ until analyzed.

\section{Experimental}

\section{A. Air Sampling Procedures}

Two to four Tenax air sample tubes at each site were collected by Air Monitoring Branch personnel using a flow of approximately $0.2 \mathrm{~L}$ minute for 120 minutes. One tube from each site was screened for CW agents using LOP \# MB-9, approved by the Center Quality Assurance Branch (CQAB) and used daily in the Air Monitoring Branch. This assay utilized Gas Chromatography (GC) with a very sensitive dual Flame Photometric Detector (FPD) allowing simultaneous detection of all the major $C W$ agents (GB, GD, VX, and $H D)$. Positive results were 
confirmed by either GC-Atomic Emission Detector (GC-AED) or GC-Mass Spectrometry (GC-MS) with a second tube. When GC-MS was used as a confirmation method, this second tube was also used to identify agent precursors, agent breakdown products, and hazardous chemicals collected on the sample tubes.

\section{B. Gas Chromatography-Flame Photometric Detector (GC-FPD) Screening Procedure}

The instruments include a Hewlett-Packard Gas Chromatograph (GC), Dynatherm Inc. ACEM 900 and other related accessories. The system receives a Depot Area Agent Monitoring System (DAAMS) solid sorbent tube, desorbs the agents from this tube onto a capillary column which separates the agents and delivers them onto a dual Flame Photometric Detector (FPD) which detects qualitatively and quantitatively the presence of these agents. The dual FPD design allows quantification of both $\mathrm{HD}$ and nerve agents simultaneously.

At the beginning of each workday, the operator checked, signed, and dated the preoperational checklist. The GC-FPD was checked daily for any error messages displayed. The operator checked all gas tanks to ensure there was enough supply of gasses for the day (no less than 300 pounds per square inch), GC and ACEM 900 parameters (cycle times for tube dry, tube heat, tube cool, trap heat; temperature settings for valves, transfer lines, tube, and trap), all temperature settings, supply of paper and ink on the integrator, if used. The operator took appropriate actions to correct any malfunctions found. This pre-operational instrument check should be recorded in the $\log$ book by the operator.

One calibration standard is prepared to be approximately 0.25 TWA for each agent in one solution. This standard is injected at incremental levels. The levels are $0.25,0.50,0.75,1.0$ and 1.25 TWA. The combination standard is used for routine operations. However, there are occasions where any combinations of standards may be used in establishing the standard curves. The selection of agents was GB, GD, VX, and HD for the customary FPD screen. The general procedures are as follows.

1. Remove agent from cold storage and place in fume hood.

2. Allow 15 minutes for agent to reach room temperature.

3. Place a previously conditioned Tenax filled sorbent tube into a Dynatherm model 10 tube conditioner. Note, flow in conditioner should be no less than $200 \mathrm{ml} / \mathrm{min}$.

4. Using a 10 microliter syringe, withdraw one microliter of agent from the agent vial.

5. Spike the tube in the tube conditioner by depressing the plunger on the syringe. Allow the spiked tube to aspirate for three minutes.

6. Remove the spiked calibration tube from the tube conditioner and place into the Dynatherm ACEM 900 tube desorber connected to a Hewlett-Packard 5890 GC equipped with a dual FPD. 
The calibration procedure is initiated by pushing the start button on the ACEM 900. Complete calibration procedure by repeating the above steps with increasing agent volumes by one microliter up to a final volume of five microliters. Normal sample analysis may begin after suitable standard curves are generated for the desired agents.

\section{Data Storage and Evaluation}

The data collected from each GC-FPD is processed and stored on a Hewlett-Packard Laboratory Information Management System (LIMS) which has a weekly tape backup routine. The chromatogram consists of peak area, peak height, retention time, and other related information. Results of analyses are checked for validity. The integrator reports the results in nanograms on column. These values are evaluated with reference to sampling time and rate of air flow during sampling, e.g. TWA level for HD is $72 \mathrm{ng}$ on column, if sampled for 120 minutes at 0.2 liters per minute air flow. If any chromatogram indicated higher than TWA level of any agent in any of the sample, a duplicate DAAMS tube is assayed using either a GC-AED (atomic emission detector) or GC-MS to confirm the result.

\section{Quality Control}

A Quality Plant (QP) sample tube that has been spiked with a known volume of $C W$ agent standard is periodically analyzed as a control sample. This QP sample, which has laboratory air aspirated through it at $0.2 \mathrm{~L} /$ minute for 120 minutes, validates the ability of the GC-FPD to detect the agent in field samples. A Quality Laboratory (QL) sample (DAAMS tube spiked with 3 ul of dilute agent standard at the 0.25 TWA level) is assayed every ten samples, as well as at the beginning and end of each day to ensure that the system is in calibration.

\section{E. CW Agent Confirmation and Analysis for Semivolatiles and Volatiles by GC-MS}

DAAMS tubes were analyzed by GC-MS using the same general procedures outlined above except the GC's detector is a Hewlett-Packard Mass Selective Detector (MSD) Model 5970B. The MSD was tuned and scanned according to the specifications of EPA Method 8270 for semivolatiles. The GC oven program was as follows: $60^{\circ} \mathrm{C}(2$ or $4 \mathrm{~min})$ at $20^{\circ} \mathrm{C} / \mathrm{min}$ to $280^{\circ} \mathrm{C}(2$ $\mathrm{min}$ ). In the GC-MS assays that used a 2 minute initial time, the overall run time is 2 minutes less and the components elute approximately 2 minutes less. This shorter initial temperature time was used on the samples that were analyzed for $\mathrm{CW}$ agent confirmation by GC-MS. The interface was at $280^{\circ} \mathrm{C}$ and the electron multiplier was at $600 \mathrm{~V}$ above tune levels. Ions used as indicators of the $\mathrm{CW}$ agents were as follows: $\mathrm{GB}$ at $\mathrm{m} / \mathrm{z} 125,99$, and $81, \mathrm{GD}$ at $\mathrm{m} / \mathrm{z} 126,99$, and $82, \mathrm{HD}$ at $\mathrm{m} / \mathrm{z} 160$, 158,111 , and 109 , and $V X$ at $\mathrm{m} / \mathrm{z} 167,127$, and 114 .

During the general characterization analysis of the Pilot Plant air samples, the MSD was in the full scan mode from 50 to 500 AMU. Component spectrum identification was made on the basis of an automated search of the Wiley Library and on visual comparison with standard spectra. Retention times and spectra of standards for the chemical warfare agents and some of the target compounds were obtained by injecting the compounds on a DAAMS tube and analyzing the tube 
in the same manner as the samples. The concentrations reported in the tables were determined by using the area response of $1 \mathrm{ng}$ of toluene on column as 7.190,000. Blanks were analyzed at the beginning of each day for contamination.

\section{Results and Discussion}

\section{A. Chemical Warfare Agent Screen}

All sites were screened for the presence of GB, GD, VX, and HD in selected DAAMS tubes by GC-FPD. Several DAAMS tubes initially appeared positive for HD (4D2-S) and GB (3D2$\mathrm{N}, 3 \mathrm{D} 2-\mathrm{S}, 3 \mathrm{ET}-\mathrm{N}, 3 \mathrm{ET}-\mathrm{S}, 4 \mathrm{D} 2-\mathrm{N}, 1 \mathrm{FB}, 1 \mathrm{FA}$, and E/205-S) at levels below the TWA reporting limits. Several samples initially appeared positive for VX $(1 \mathrm{C}-1,1 \mathrm{C}-2,1 \mathrm{D}-1$, and $1 \mathrm{I}-1)$ at or above the TWA reporting level. In an attempt to confirm these low levels, other tubes from the same sites were analyzed by GC-MSD or GC-AED. None of the initial GC-FPD positive results could be confirmed by GC-MSD or GC-AED; therefore, the overall results were negative for the agents analyzed as seen in Table 1 . The probable cause of the false positives was a high level or organic interference seen during the GC-MSD full scan analysis of the air samples for non-agent hazardous materials. The identity of some of these interferences, which included phosphorous and sulfur containing compounds, will be discussed below.

One additional sample was received from Room 134, also called the " $V$ " room. This sample represented the collection of $660 \mathrm{~L}$ of air by a DAAMS tube. The purpose was to look for trace amounts of mustard, none of which was detected. The method detection limit in this case was 1.5 $\mathrm{pg} / \mathrm{L}$. The negative finding should be cautiously interpreted because the high sample volume could cause break through.

\section{B. Hazardous Chemical Screen}

Sample tubes were screened for volatile and semivolatile chemicals by GC-MS using either a 2 or 4 minute initial temperature time. The 2 minute initial time was used for samples that were originally analyzed for confirmation of $\mathrm{CW}$ agent contamination. Samples analyzed using the 2 minute hold time yield components with lower retention times and an overall run time of 14.5 minutes. The decision to change to a 4 minute initial time was based on the need to increase separation early in the chromatographic assay. This need was driven by the presence of compounds such as trichlorofluoromethane, hexane, and trichloroethylene. Four minutes also allows the contents of the focusing trap of the Dynatherm to be completely evacuated onto the capillary column before the GC oven temperature is ramped. Results are shown in the Appendix 1 (Figures 1-26) for representative ion chromatograms and Appendix 2 (Tables 2-14) for a list of possible components. These tables are not inclusive and other components could be hidden in the complex data.

The total ion chromatograms indicated the complexity of the data recovered from analysis of the DAAMS tubes. Many peaks seen in the chromatograms represented substances that were not listed as possible contaminants by a historical report done by Argonne National Laboratory on the Pilot Plant (see Enclosure 1). The identity of some of these chemicals has yet to be determined as 
noted by the "unknown" under the compound name. Besides the master table of contents for each sample and the total ion chromatogram, there are two groups of ion chromatograms that indicate the possible presence of selected compounds. Figures with ion chromatograms of $\mathrm{m} / \mathrm{z} 294,292$, and 290 were generated to highlight the presence of polychlorinated biphenyls (PCBs) in the samples. The concentration was calculated by comparing the area found for an analyte to that of the area response of $1 \mathrm{ng}$ of toluene. This yielded very approximate quantities of analyte on column which was divided by the volume of air sample collected $(24 \mathrm{~L})$ to $\mathrm{pg} / \mathrm{L}$ of air.

Several chemicals found in the general GC-MS assay could readily interfere in the standard GC-FPD screen for CW agents. These include 1,4-oxathiane, O,O-diethyl-S-phosphorothioate, and phosphoric acid triethyl ester which have either sulfur, phosphorus, or both atoms that are the targets of the FPD. These chemicals or closely related compounds are possible sources of the false positives in the standard screen.

Most of the compounds that eluted during the first 8 to 10 minutes are very volatile. They are not due to contamination from the analysis facility since the laboratory blanks were void of most of these compounds with certain exceptions. The following chemicals were found in the blanks at what appeared to be lower levels than were seen in the samples and were included in the tables: hexane, benzaldehyde, benzoic acid, limonene, diethyl phthalate, dioctyl phthalate, and hexadecanoic acid. There were several alkyl acids, column bleed components, and hydrocarbons that were omitted from the tables because they were common to both samples and blanks at similar levels.

Polychlorobiphenyls (PCBs) were seen in all samples to some degree as evidenced by the extracted ion chromatograms showing masses $\mathrm{m} / \mathrm{z} 294,292$, and 290 for each sample. In all cases, levels were in the part per trillion level. However, these quantities were determined by highly approximate methods. The actual amounts may be considerably different than reported in this study. A more cautious evaluation of the PCB levels should be undertaken using stricter criteria to determine the concentration. Also, future studies of the higher boiling components such as PCBs should take into account the sample collection temperature. An air sample study conducted in the cold of winter will yield levels that are lower than that of a summer study. However, for the lower boiling point components the opposite should be true. Colder temperature will allow the compounds to be more effectively trapped by the tenax filled DAAMS tubes. 
TABLE 1. RESULTS OF GC-FPD SCREEN FOR CW AGENTS

\begin{tabular}{|c|c|c|c|c|c|}
\hline $\begin{array}{l}\text { Sampling } \\
\text { Location }\end{array}$ & $\begin{array}{c}\text { Laboratory } \\
\text { Tube \# }\end{array}$ & GB & GD & vX & HD \\
\hline $3 \mathrm{D} 2-\mathrm{N}$ & 0116 & negative & negative & negative & negative \\
\hline $3 D 2-S$ & 0901 & negative & negative & negative & negative \\
\hline $3 \mathrm{ET}-\mathrm{N}$ & 0028 & negative & negative & negative & negative \\
\hline $3 E T-S$ & 0957 & negative & negative & negative & negative \\
\hline $4 \mathrm{D} 2-\mathrm{N}$ & 0133 & negative & negative & negative & negative \\
\hline 4D2-S & 0071 & negative & negative & negative & negative \\
\hline $4 F T-N$ & 0357 & negative & negative & negative & negative \\
\hline 4FT-S & 0599 & negative & negative & negative & negative \\
\hline $4 \mathrm{C}-\mathrm{N}$ & 0601 & negative & negative & negative & negative \\
\hline 4C-S & 0055 & negative & negative & negative & negative \\
\hline $4 A-N$ & 0715 & negative & negative & negative & negative \\
\hline 4A-S & 0769 & negative & negative & negative & negative \\
\hline $1 F B$ & 0951 & negative & negative & negative & negative \\
\hline IFA & 0906 & negative & negative & negative & negative \\
\hline $1 C-1$ & 0918 & negative & negative & negative & negative \\
\hline $1 C-2$ & 0935 & negative & negative & negative & negative \\
\hline 1D-1 & 0948 & negative & negative & negative & negative \\
\hline $\mathrm{E} / 205-\mathrm{N}$ & 0955 & negative & negative & negative & negative \\
\hline E/205-S & 0947 & negative & negative & negative & negative \\
\hline $11-1$ & 0333 & negative & negative & negative & negative \\
\hline $11-3$ & 0947 & negative & negative & negative & negative \\
\hline 132 & 0912 & negative & negative & negative & negative \\
\hline
\end{tabular}




\section{APPENDIX 1:}

FIGURES 1 THROUGH 26 
FIGURE 1: TOTAL ION CHROMATOGRAM OF AIR SAMPLE FROM 3D2, TUBE 0942

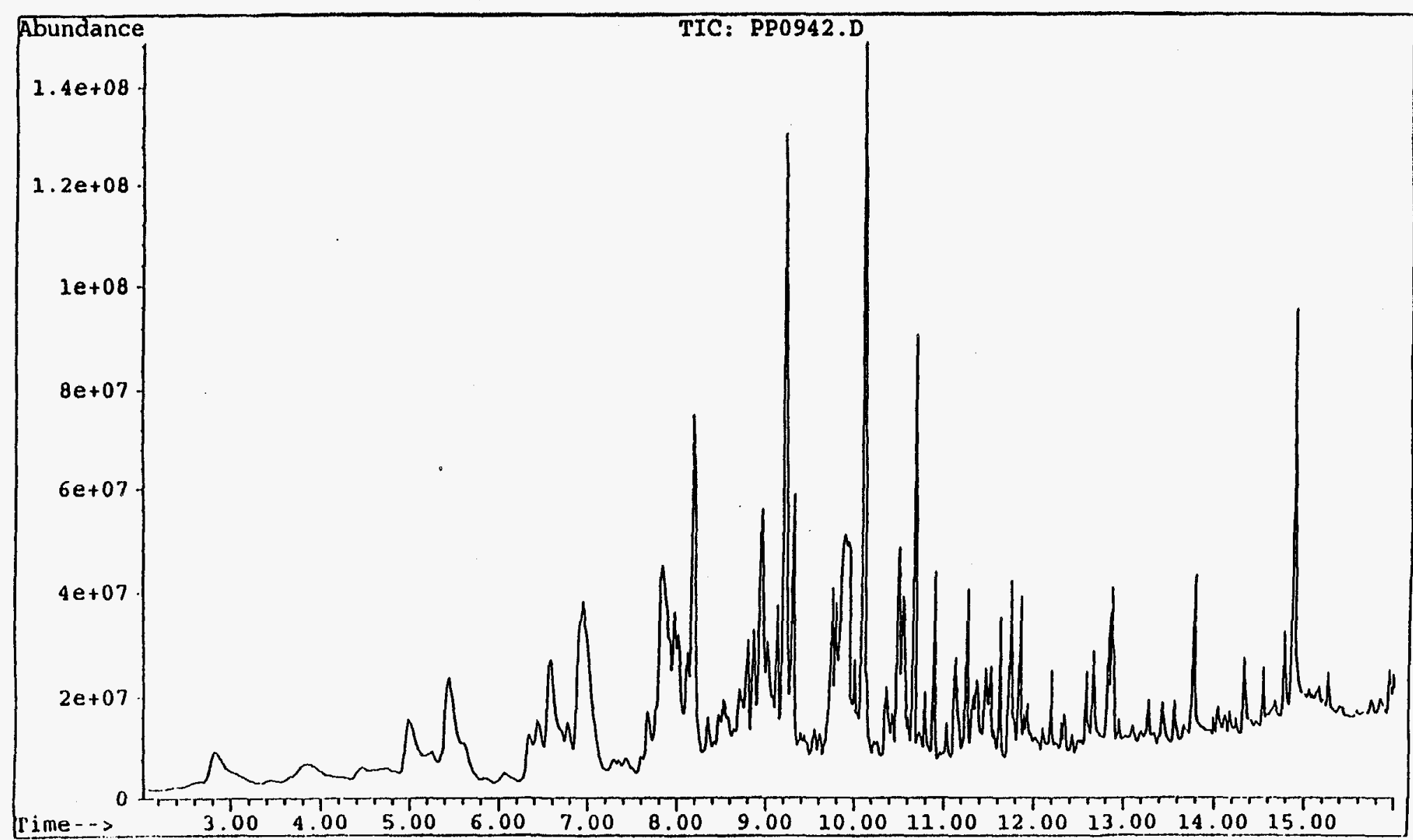




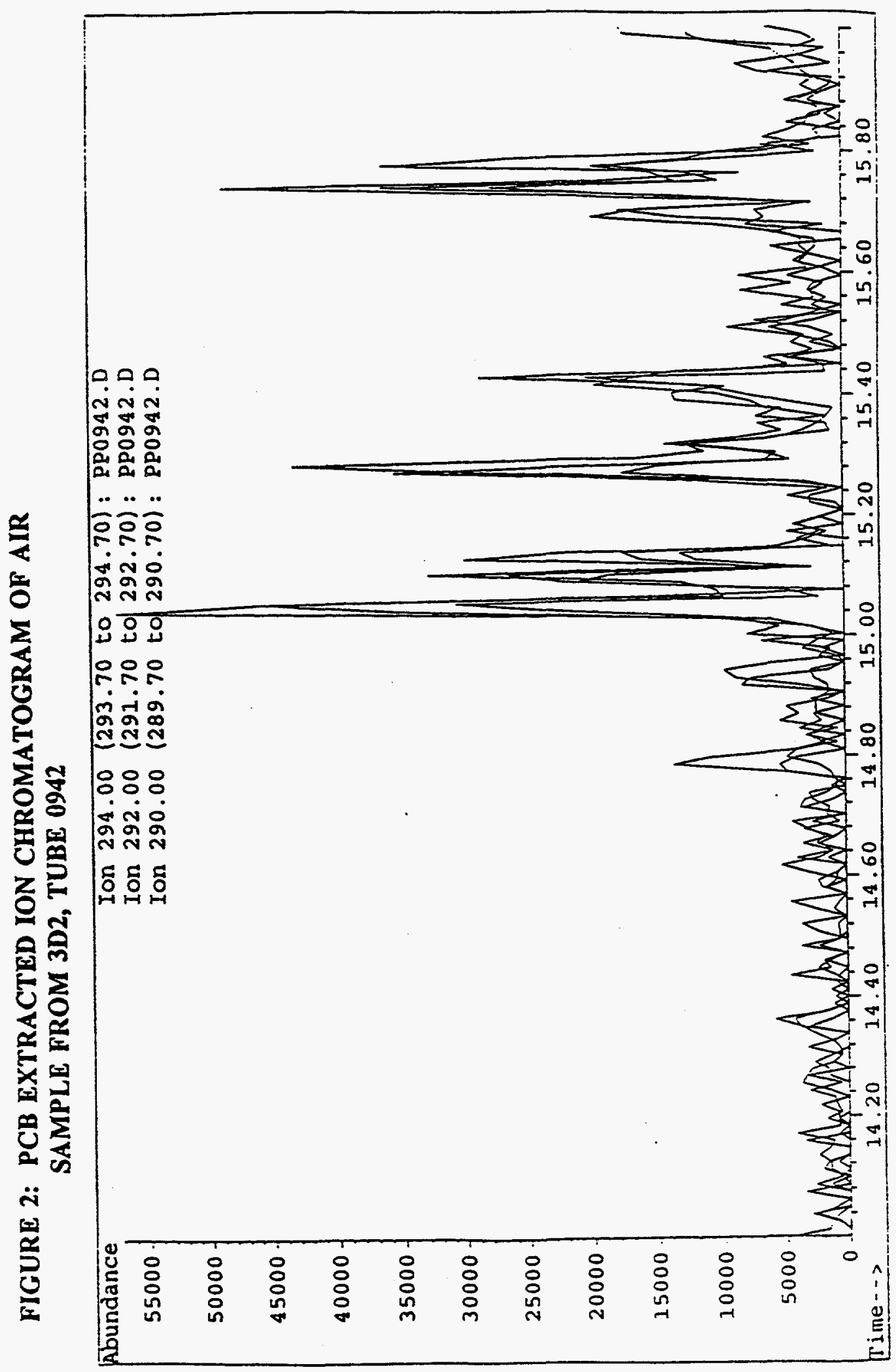




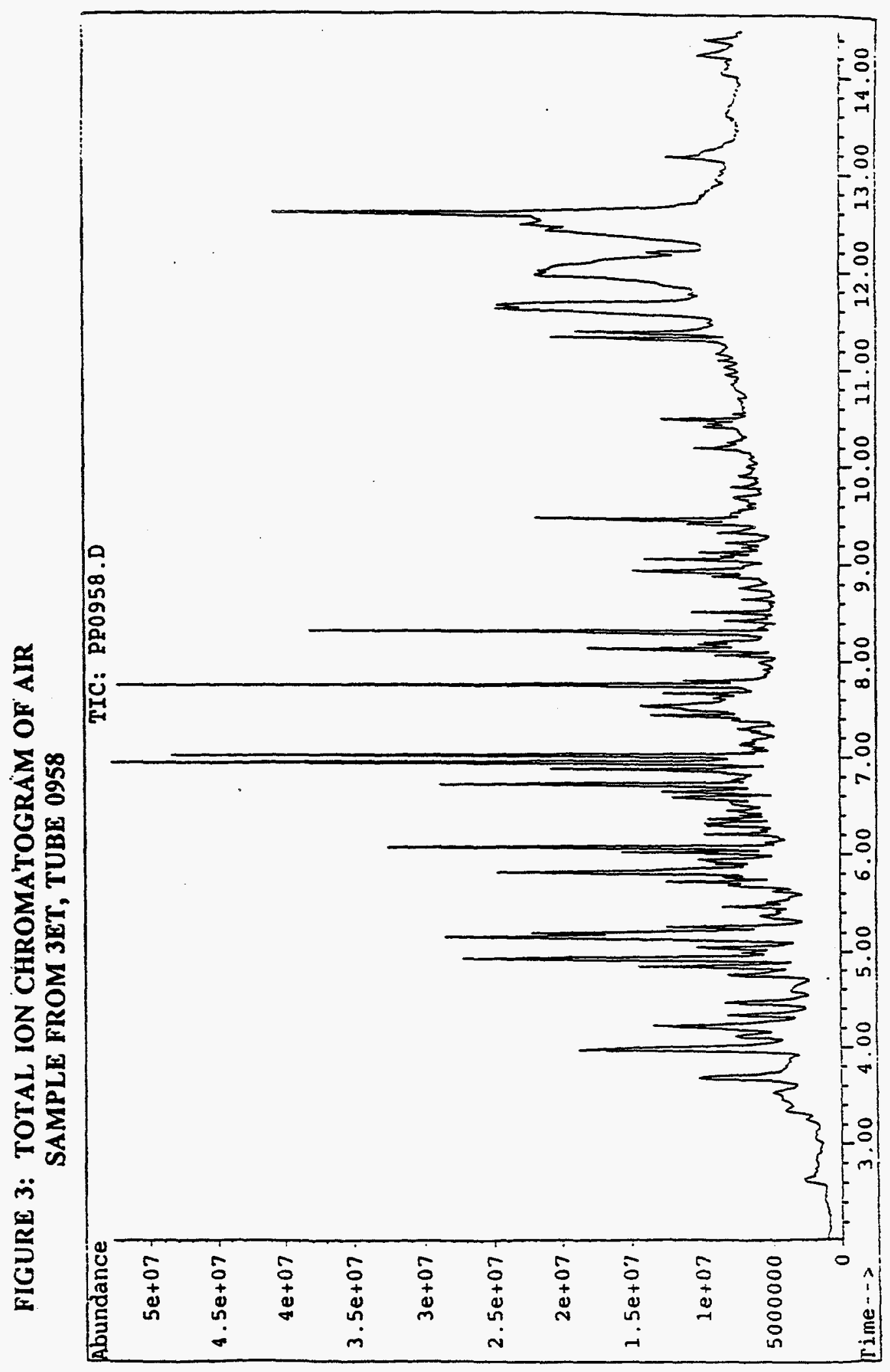




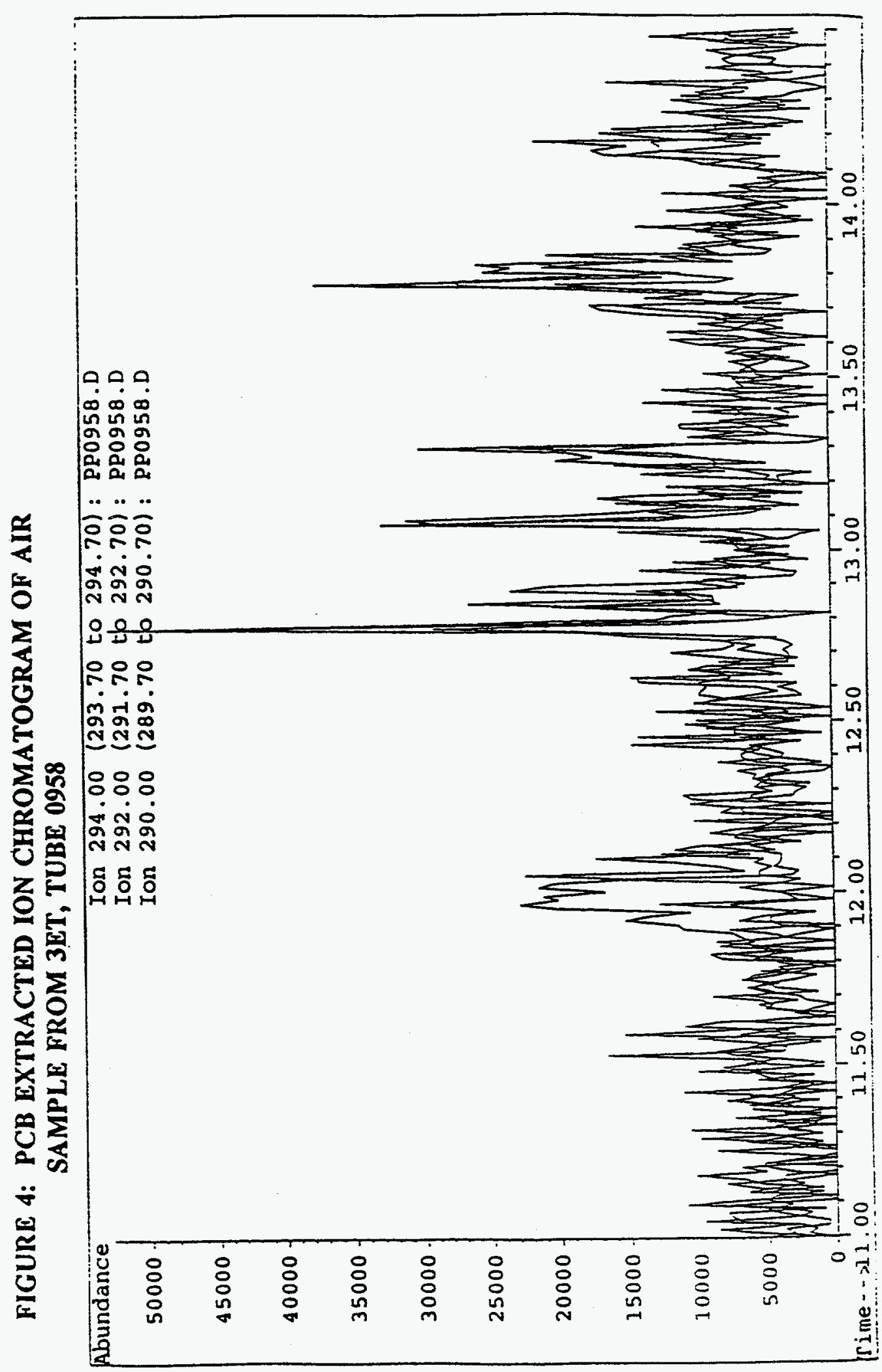




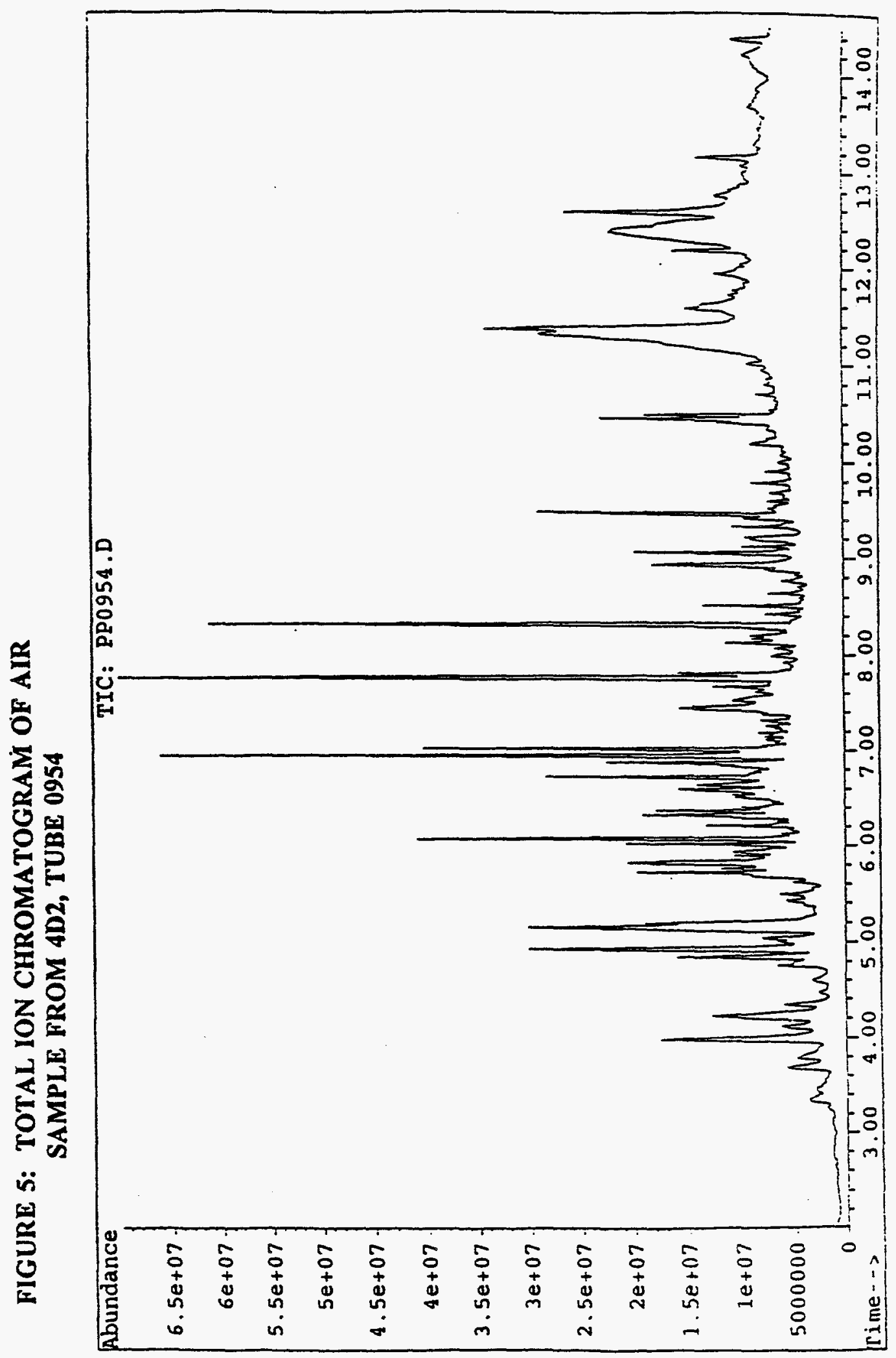




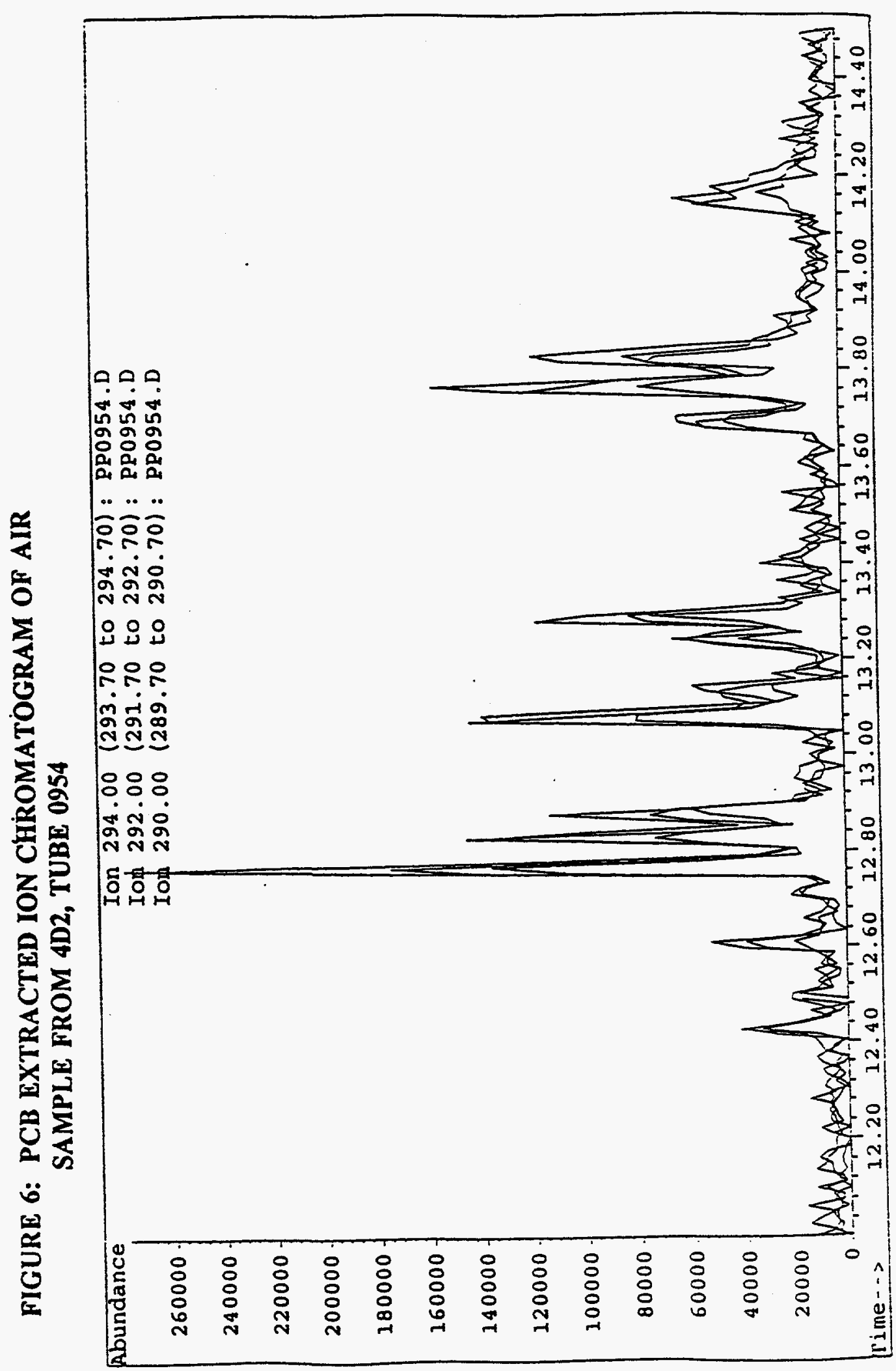




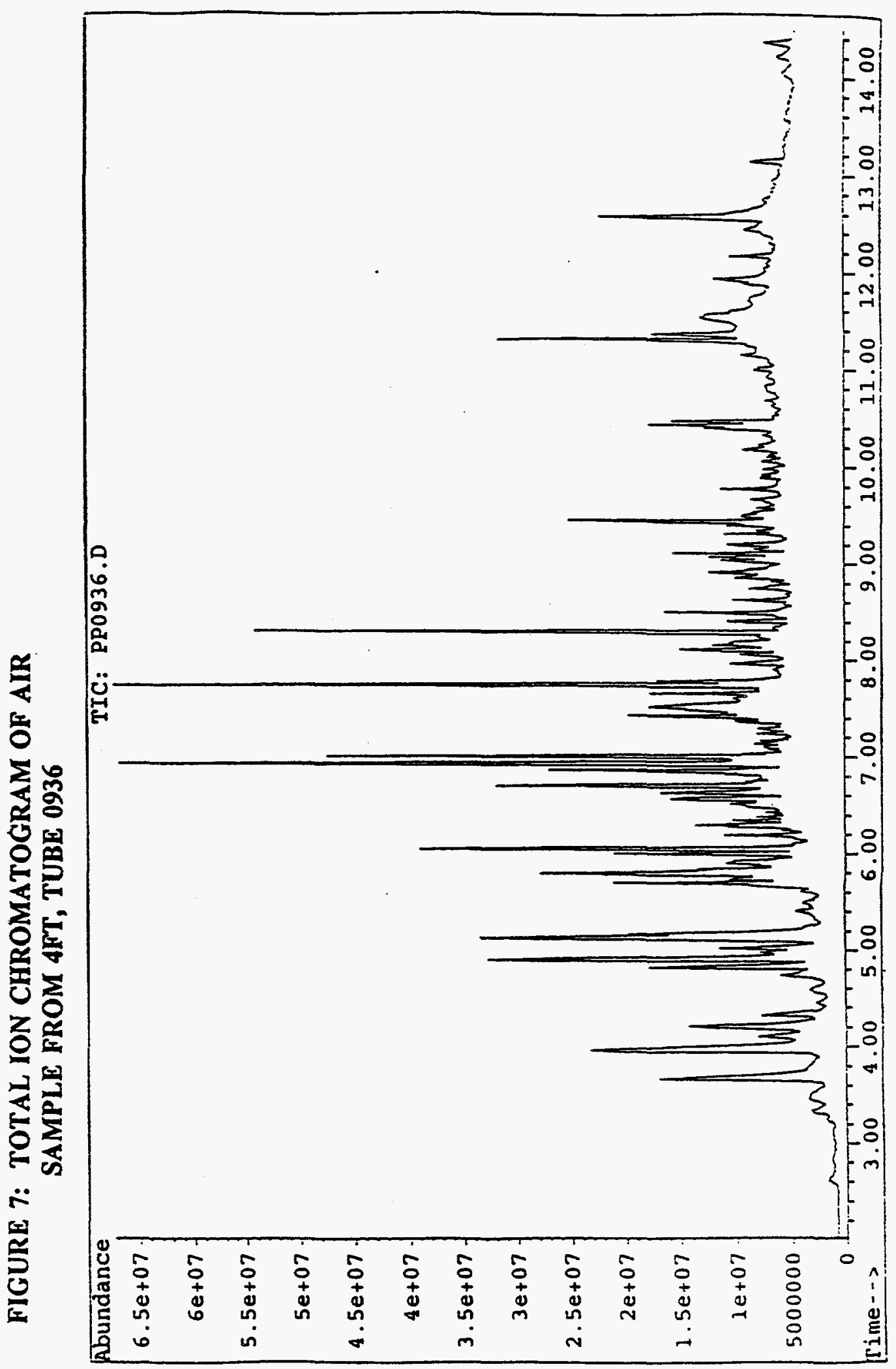




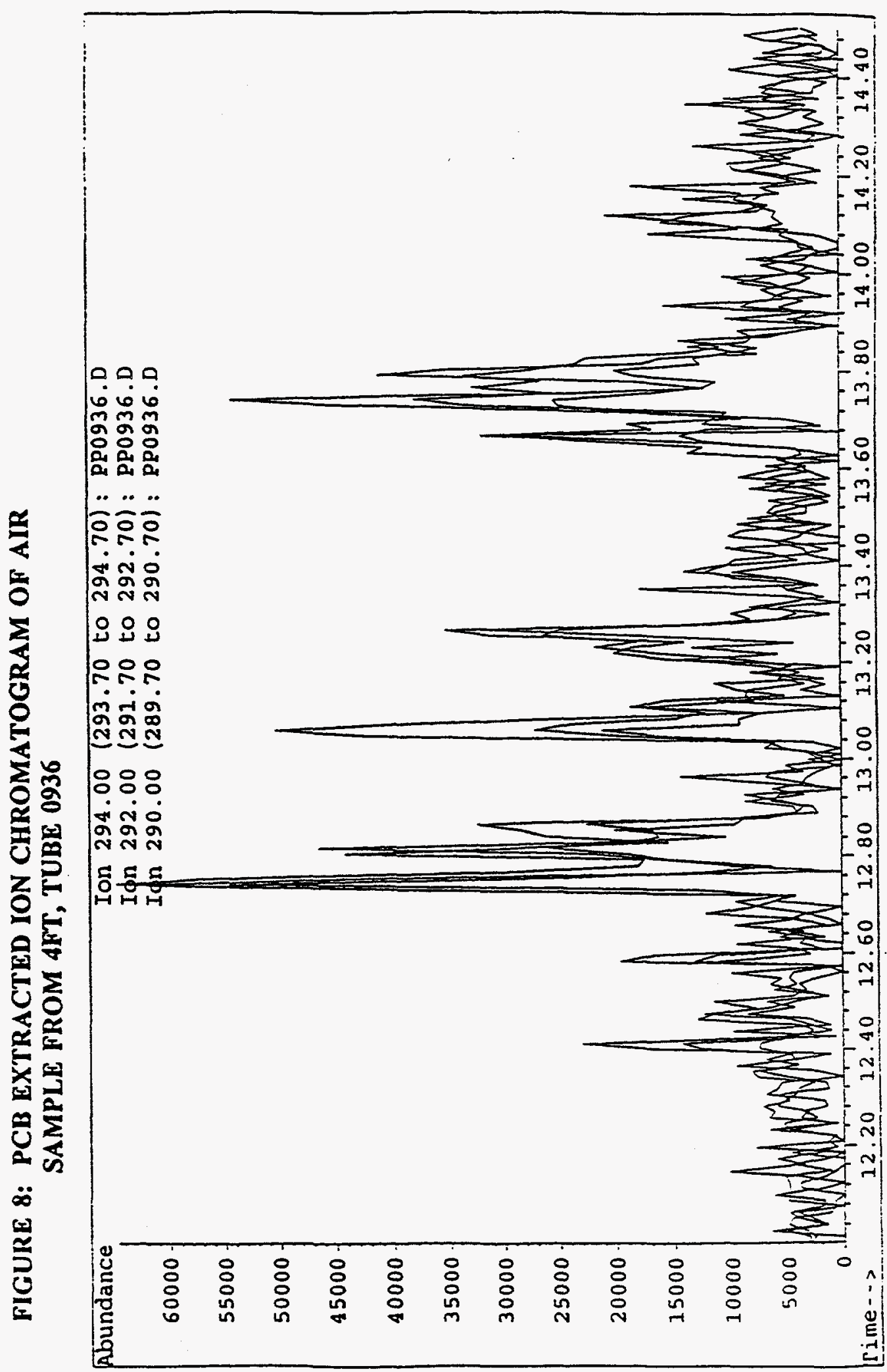


FIGURE 9: TOTAL ION CHROMATOGRAM OF AIR

SAMPLE FROM $4 C$, TUBE 0714

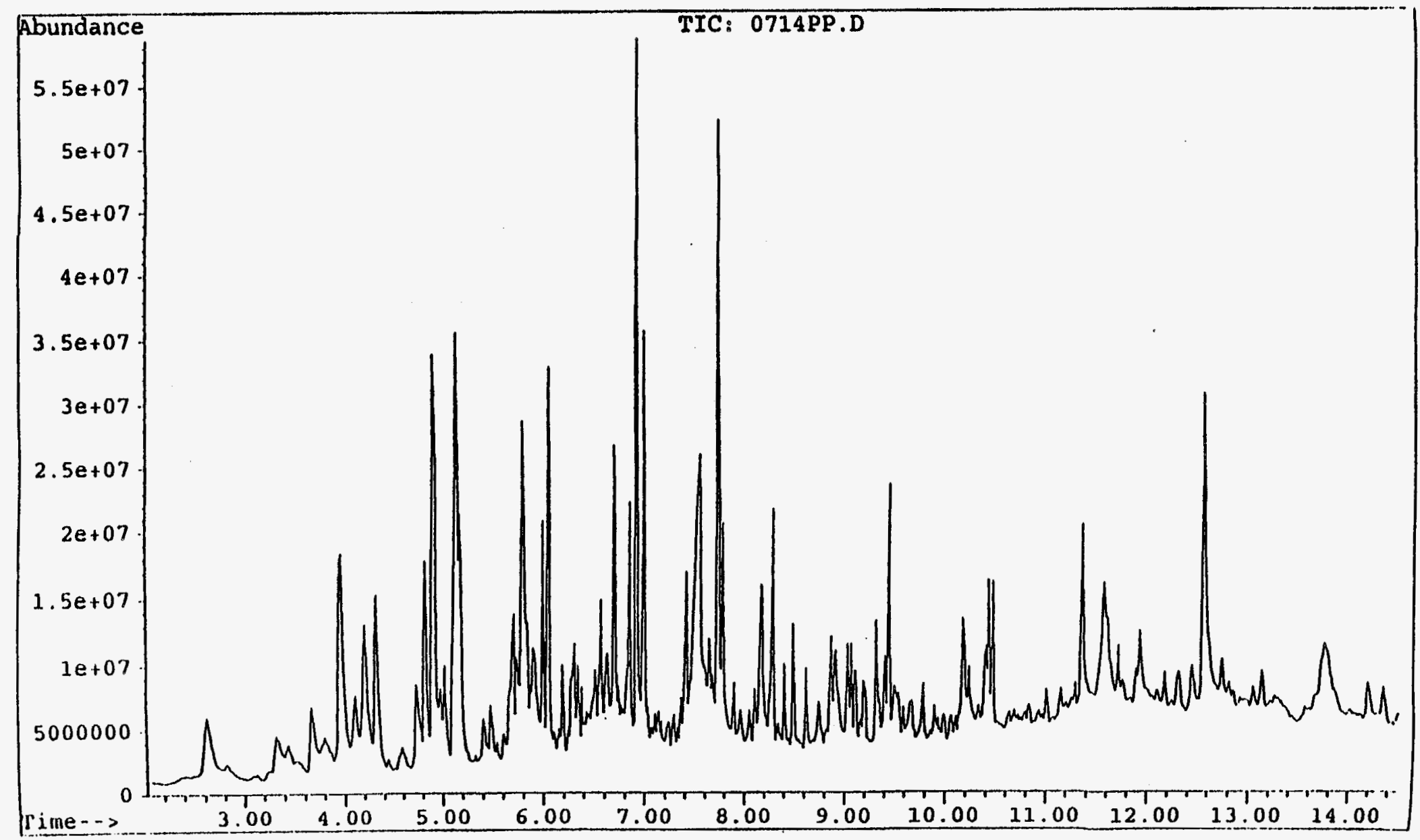




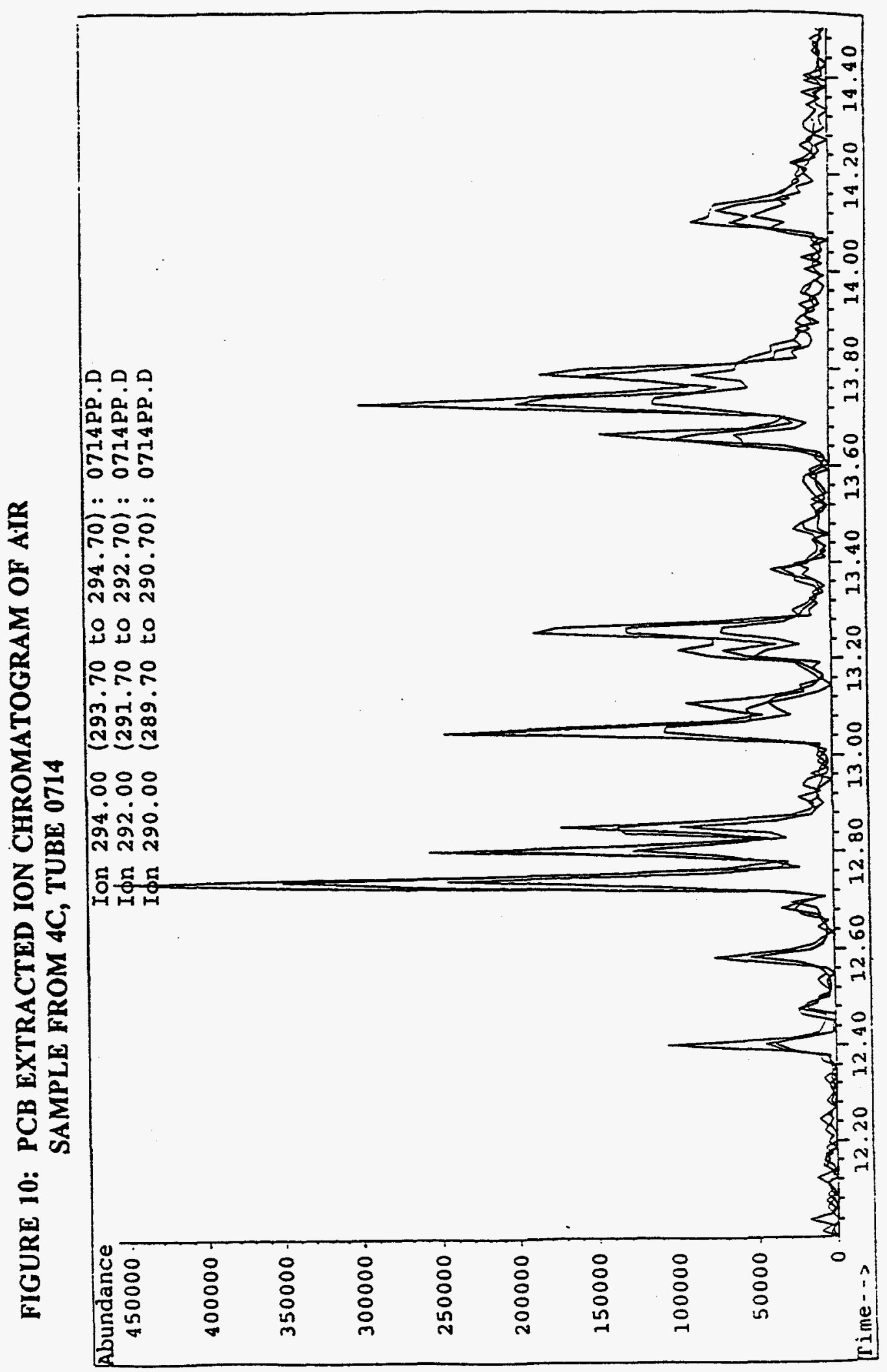




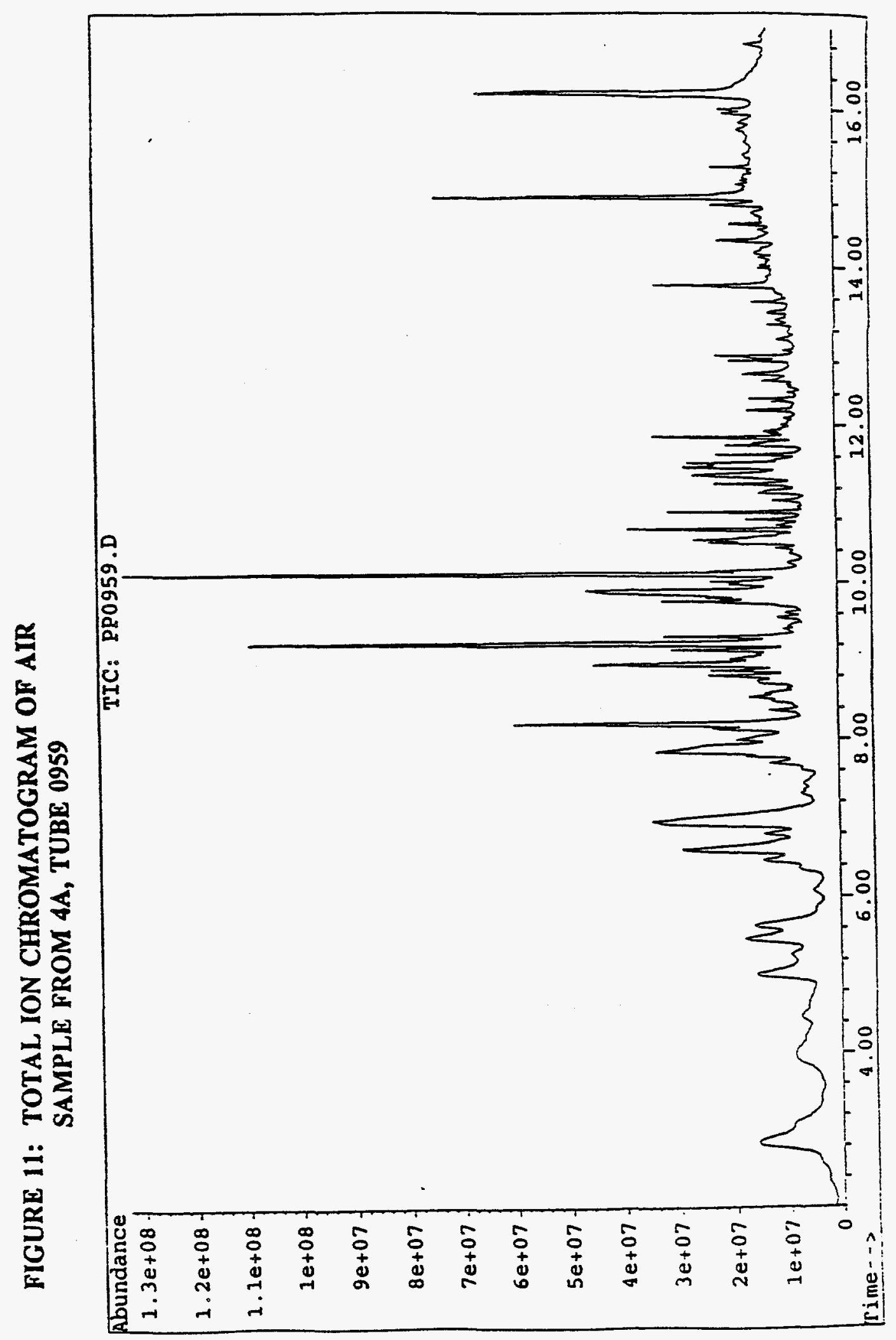




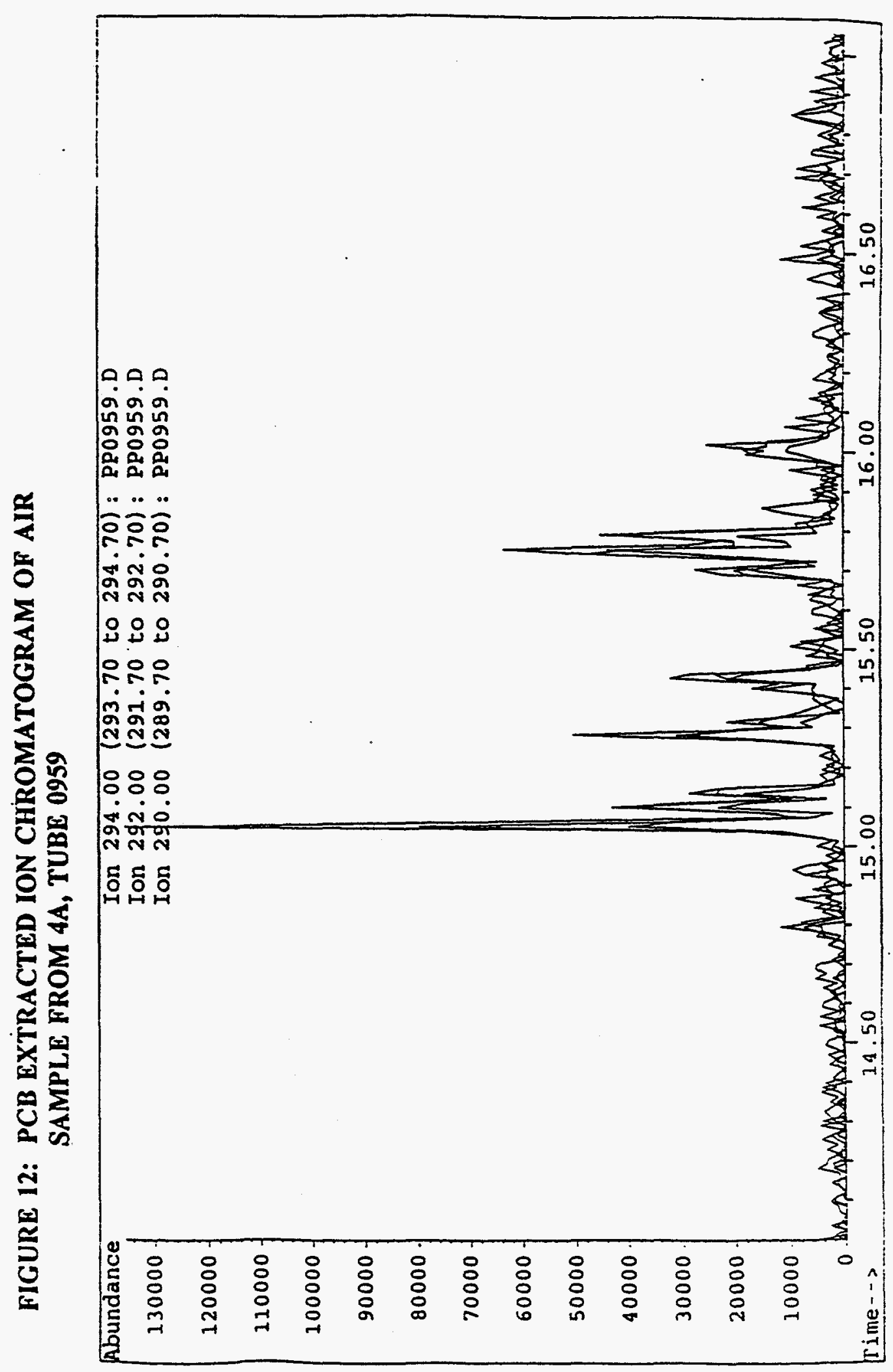




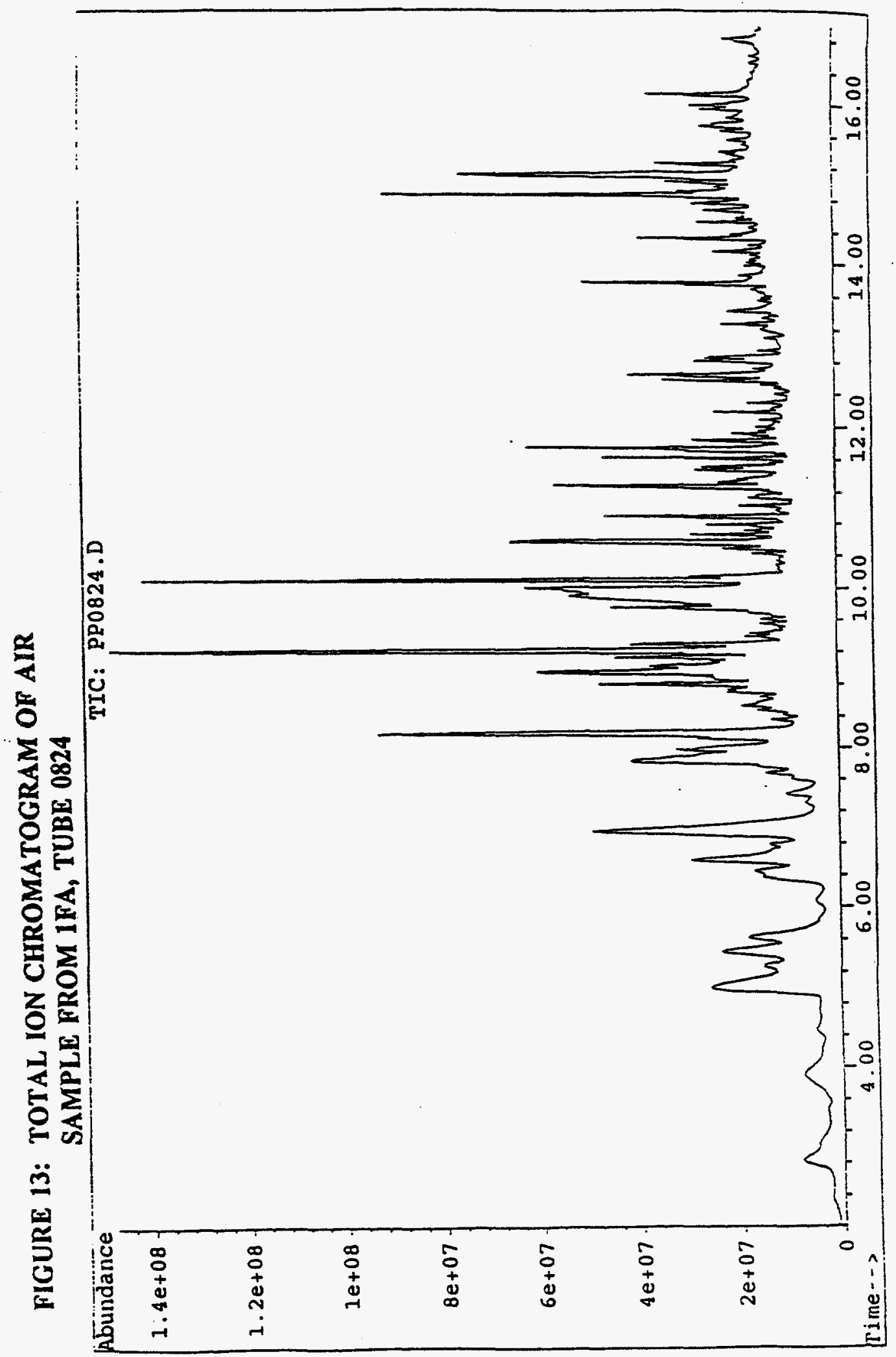




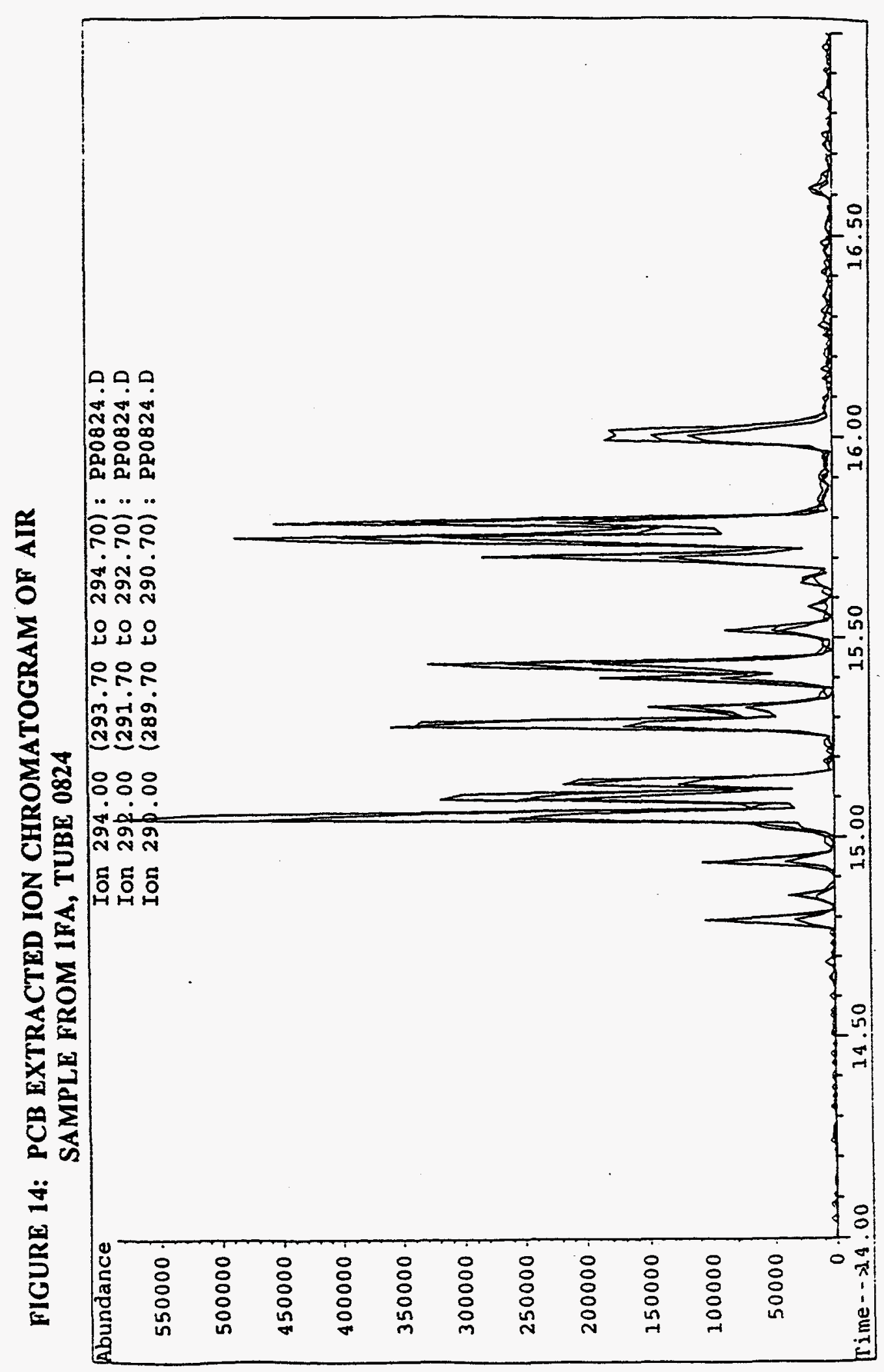


FIGURE 15: TOTAL ION CHROMATOGRAM OF AIR SAMPLE FROM 1C2, TUBE 0963

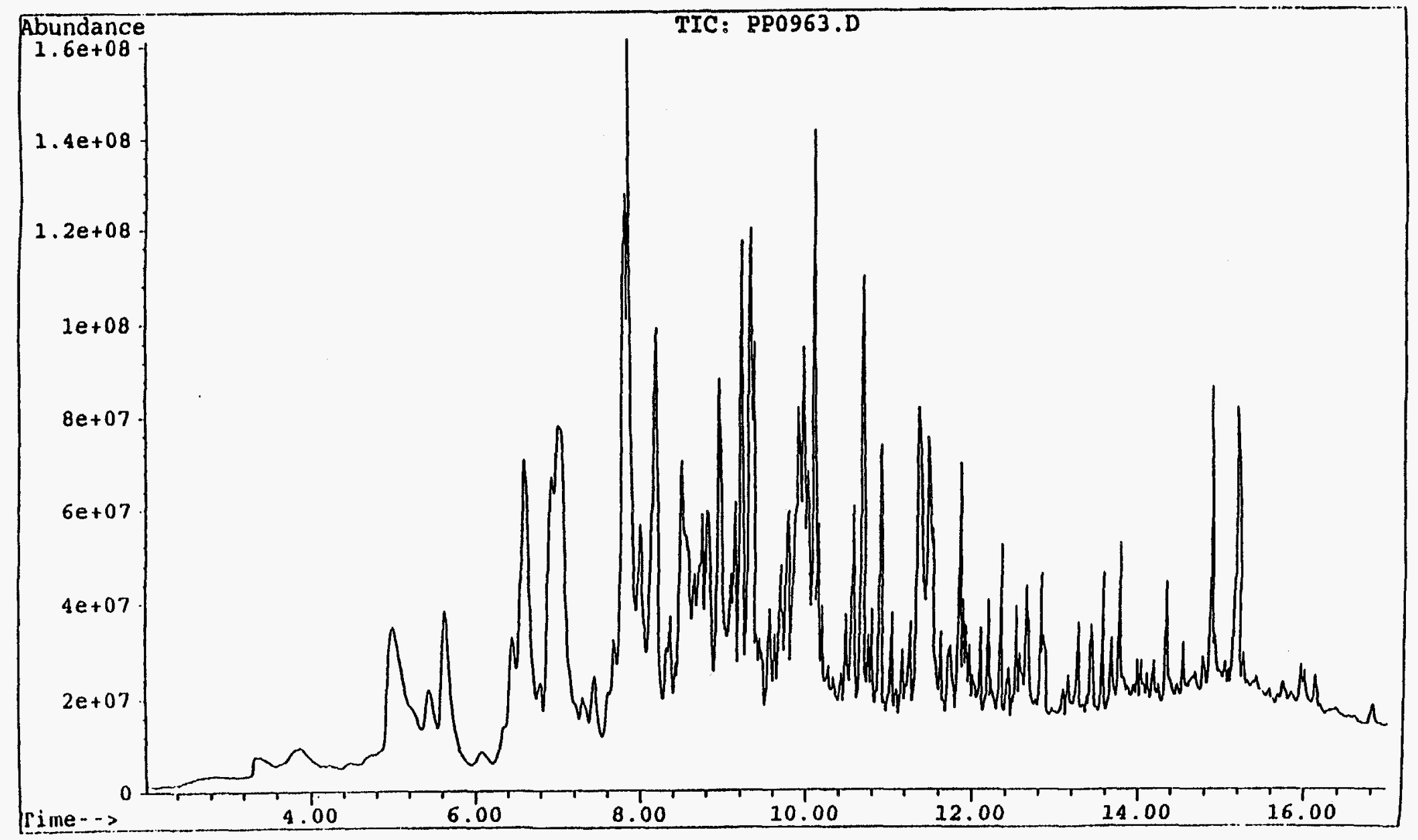




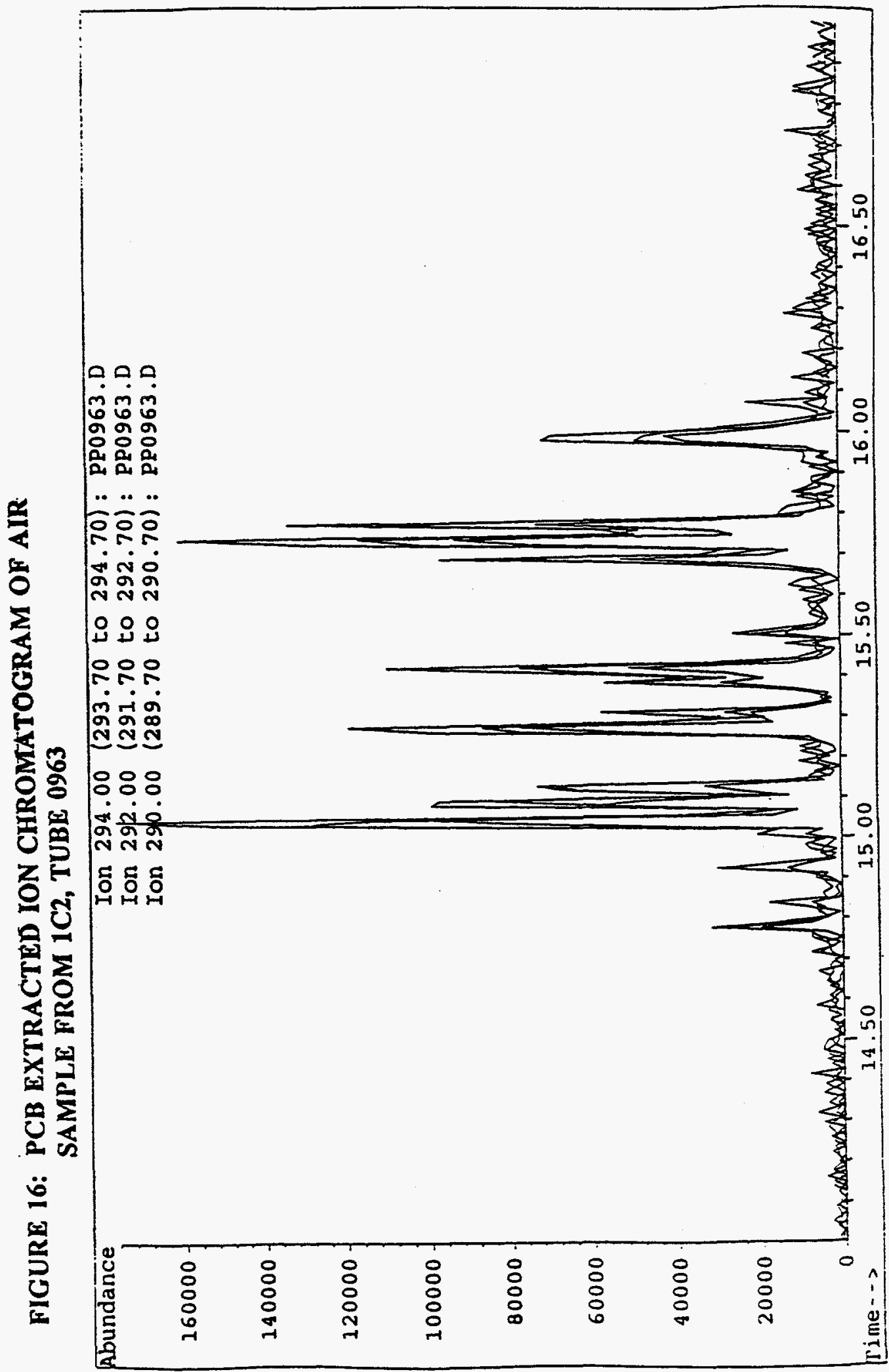




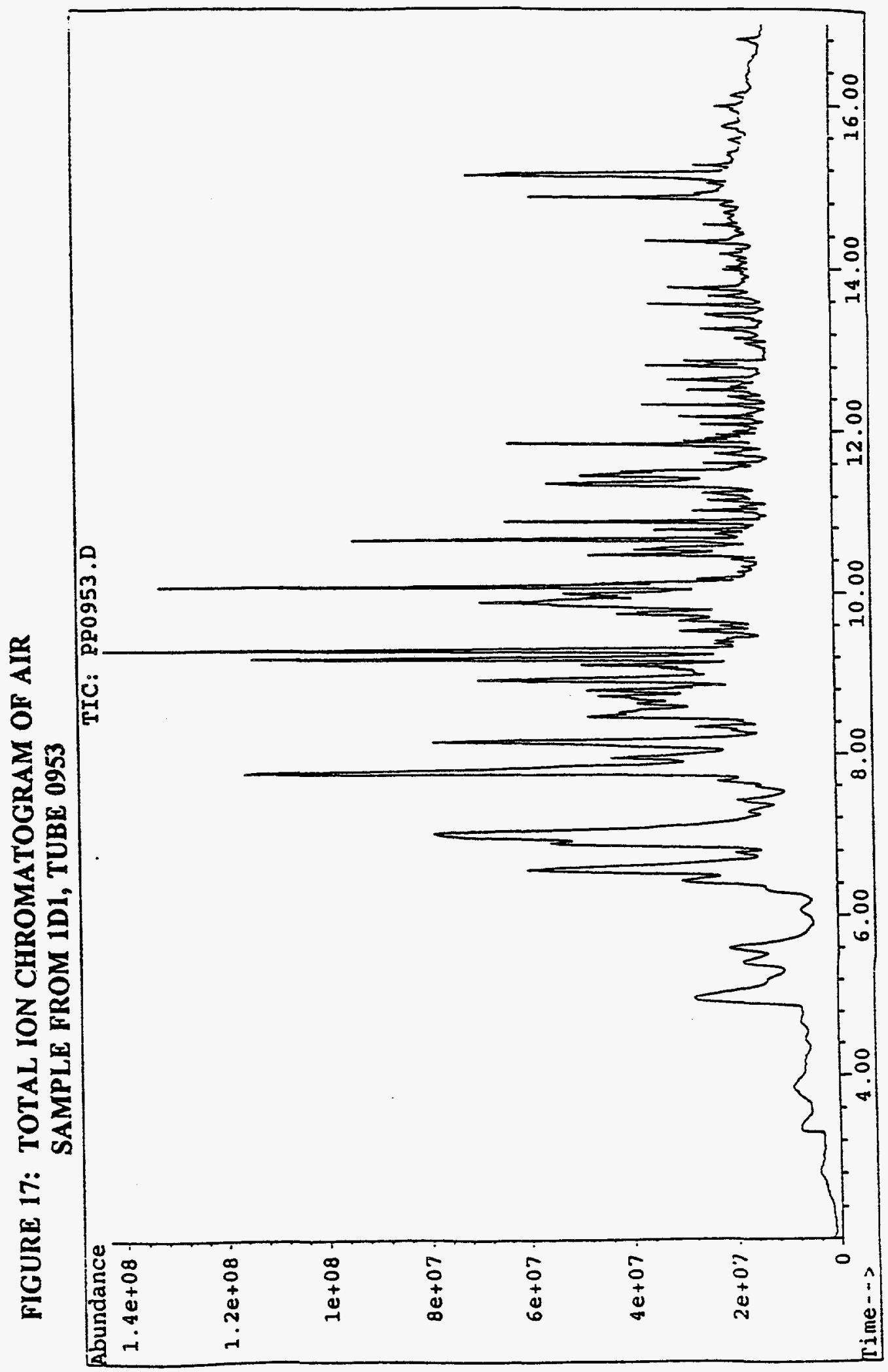




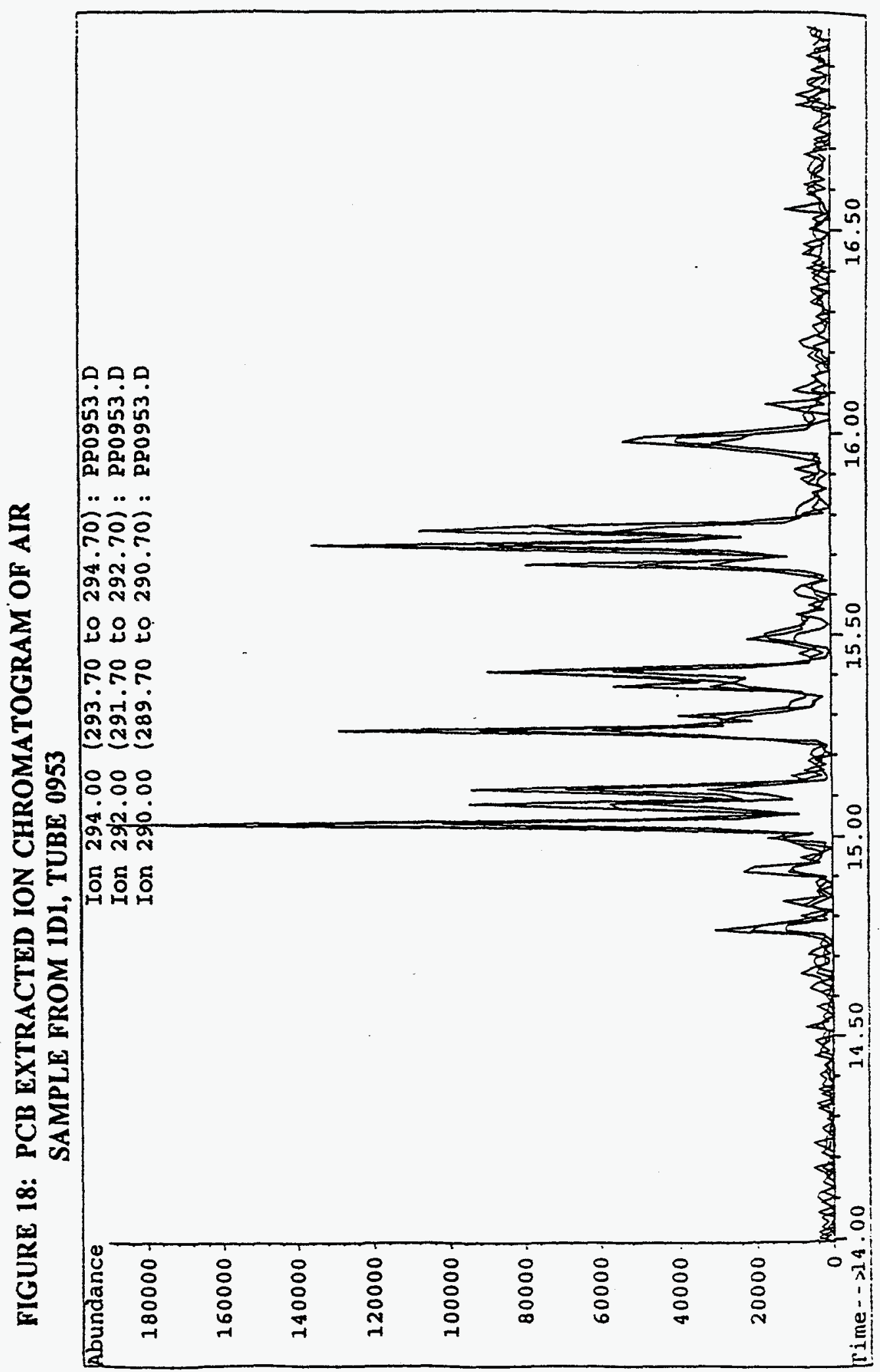




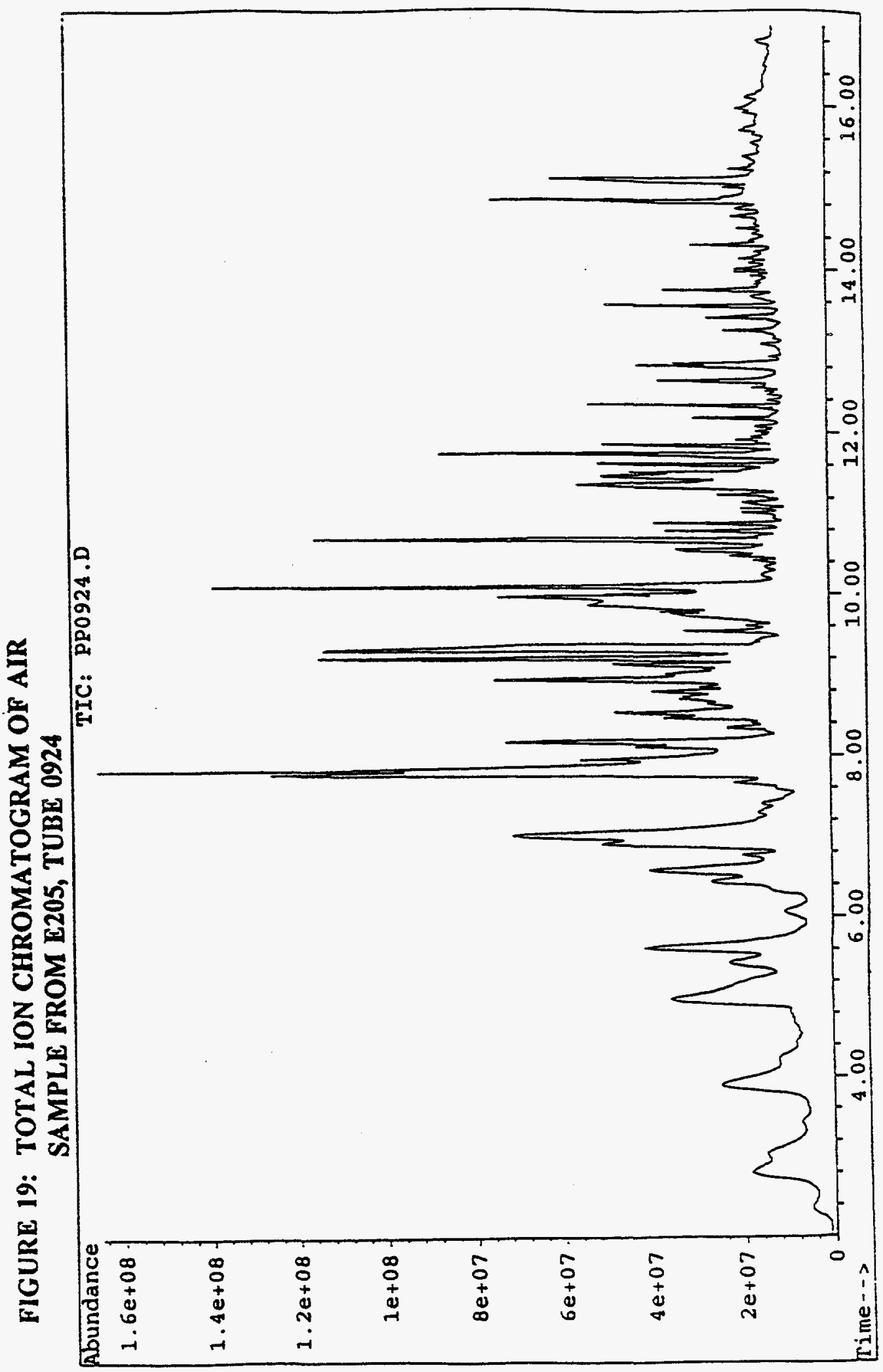


FIGURE 20: PCB EXTRACTED ION CHROMATOGRAM OF AIR S.AMPLE FROM E205, TUBE 0924

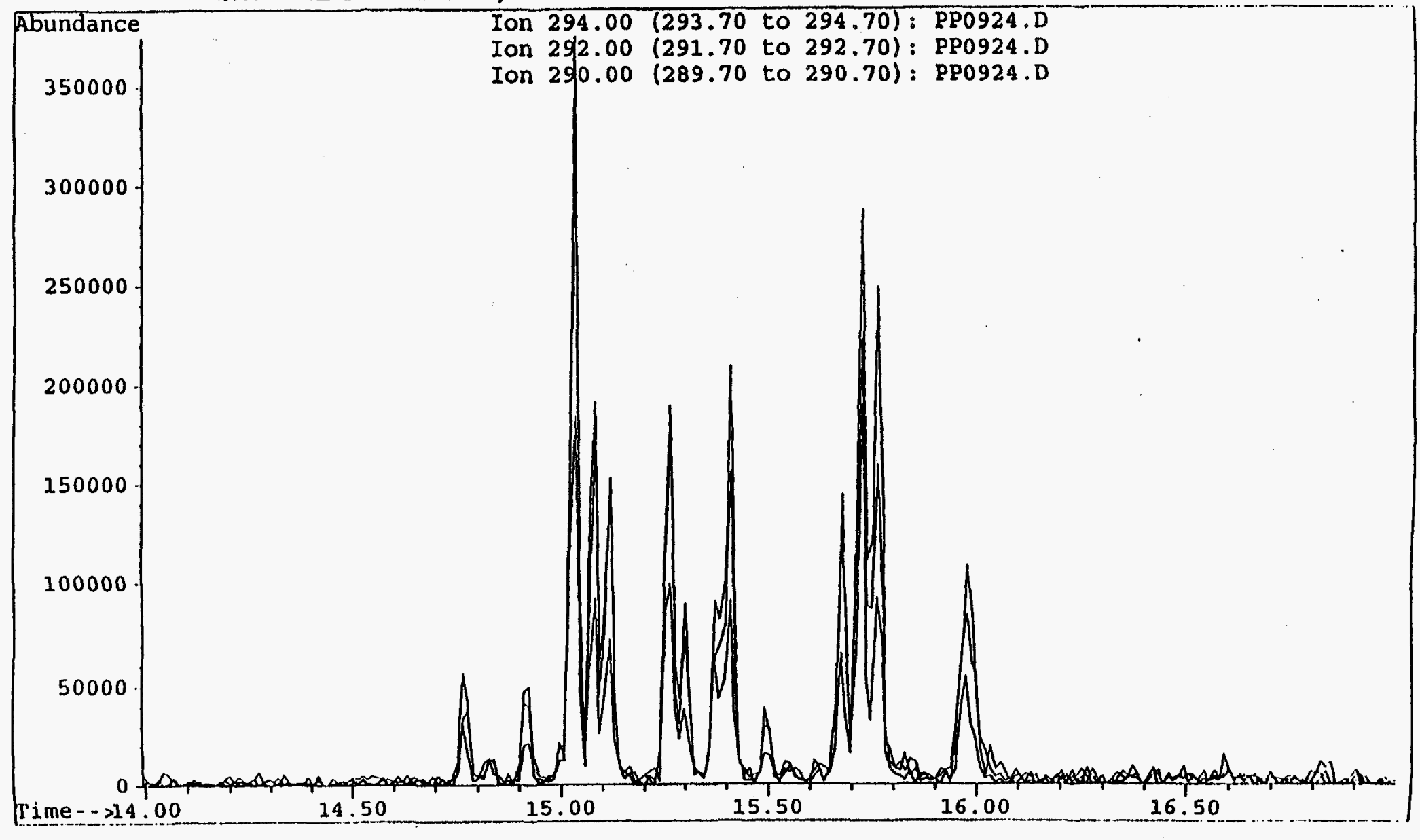




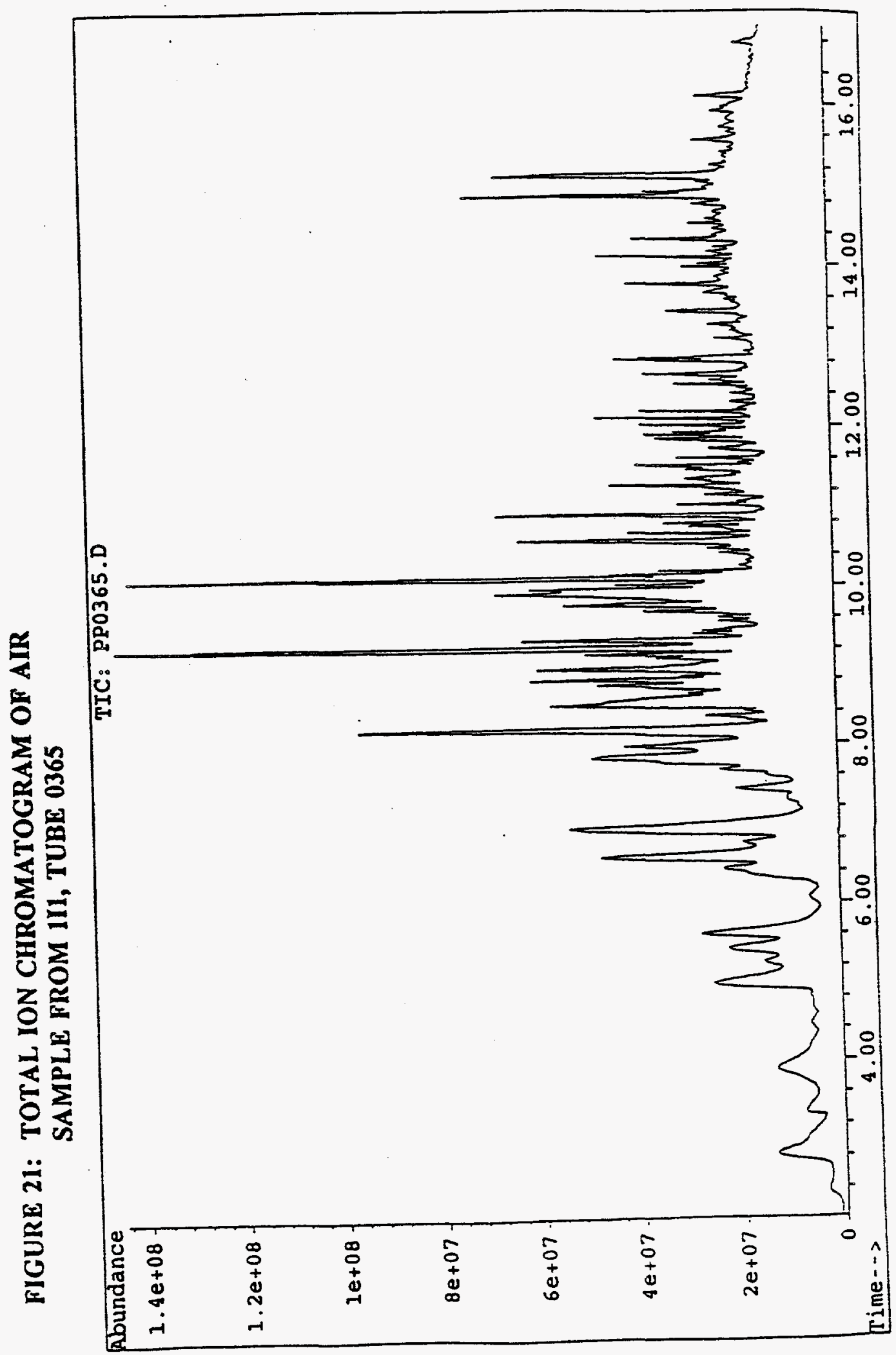




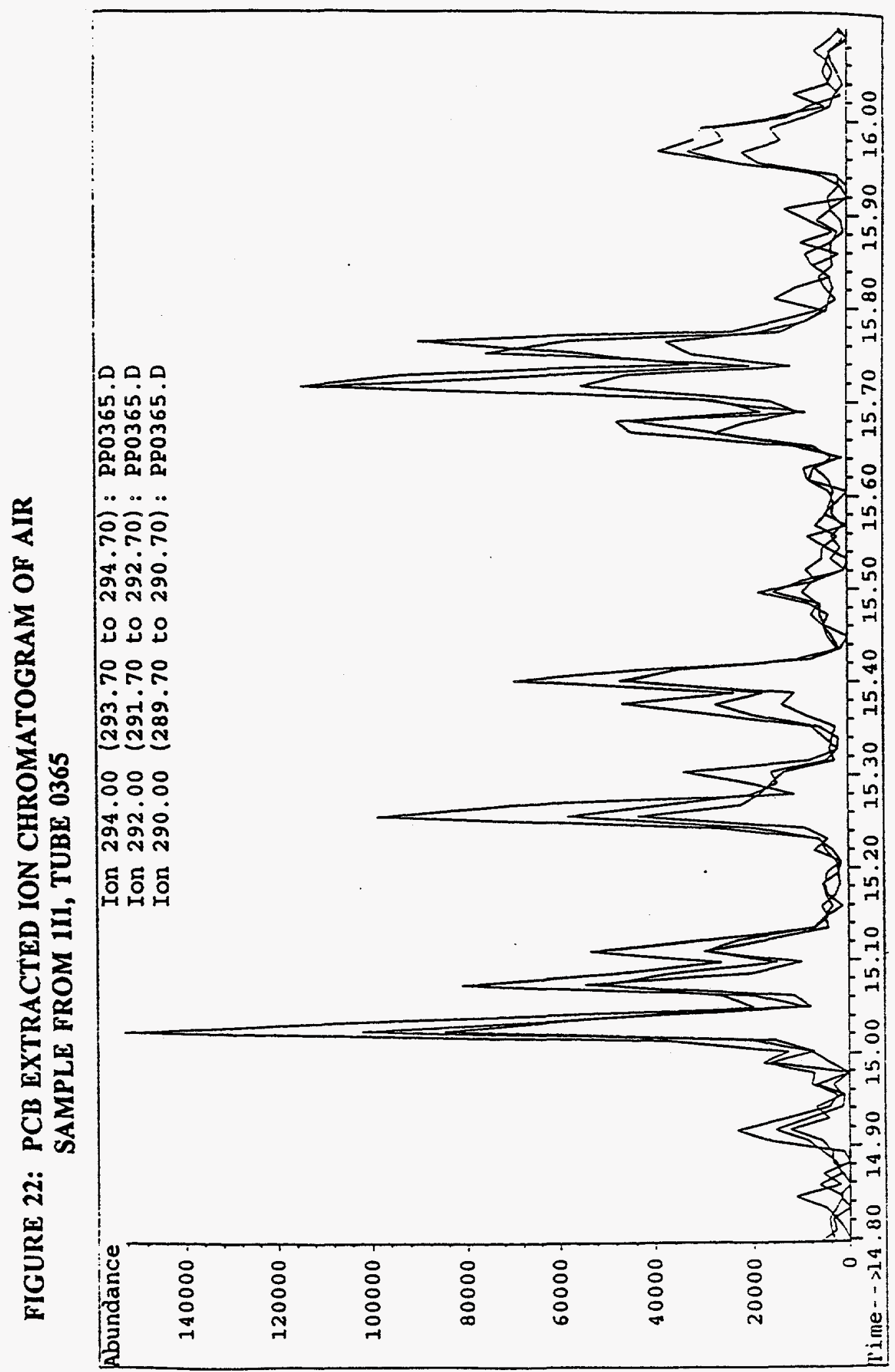




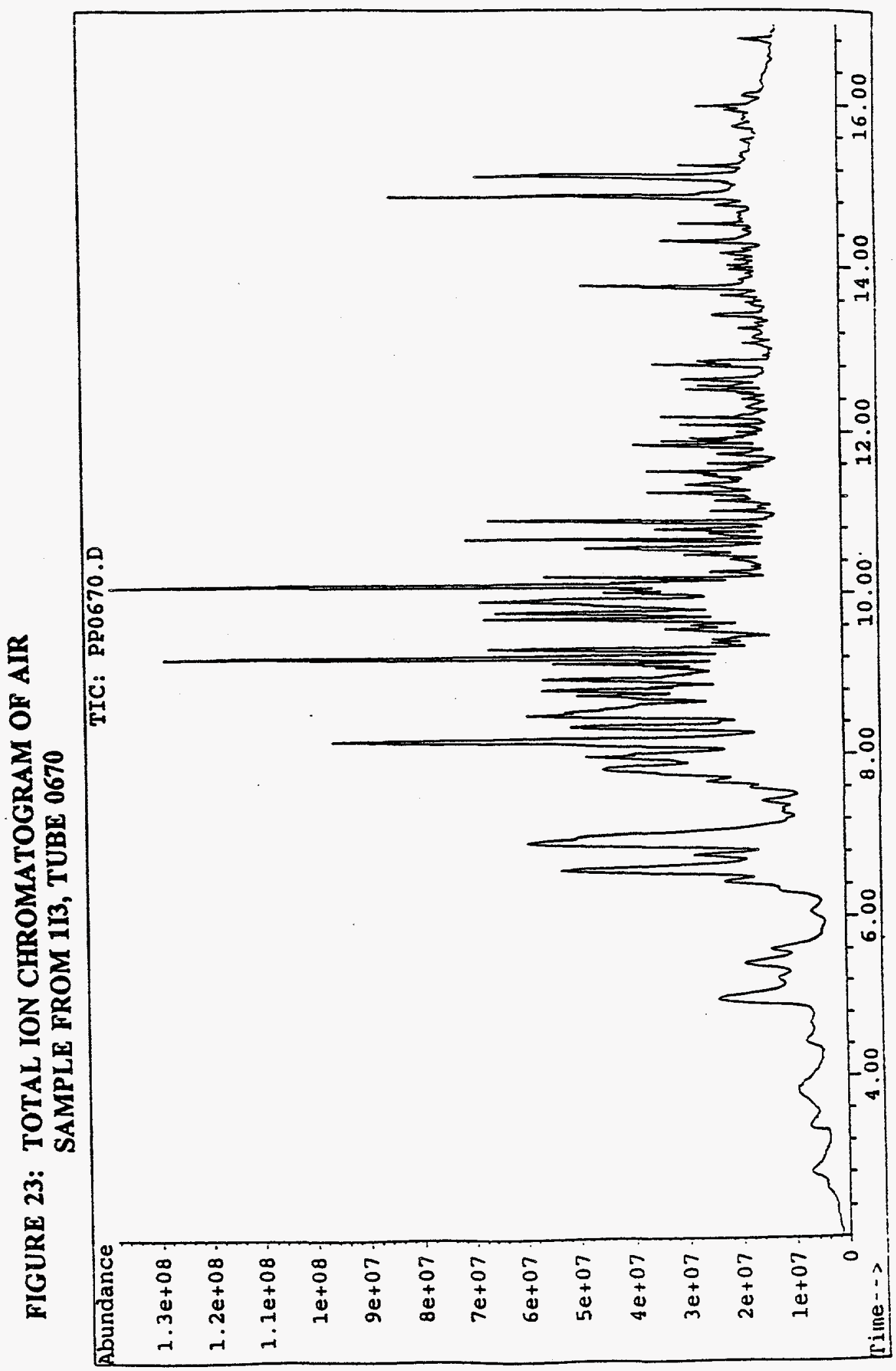




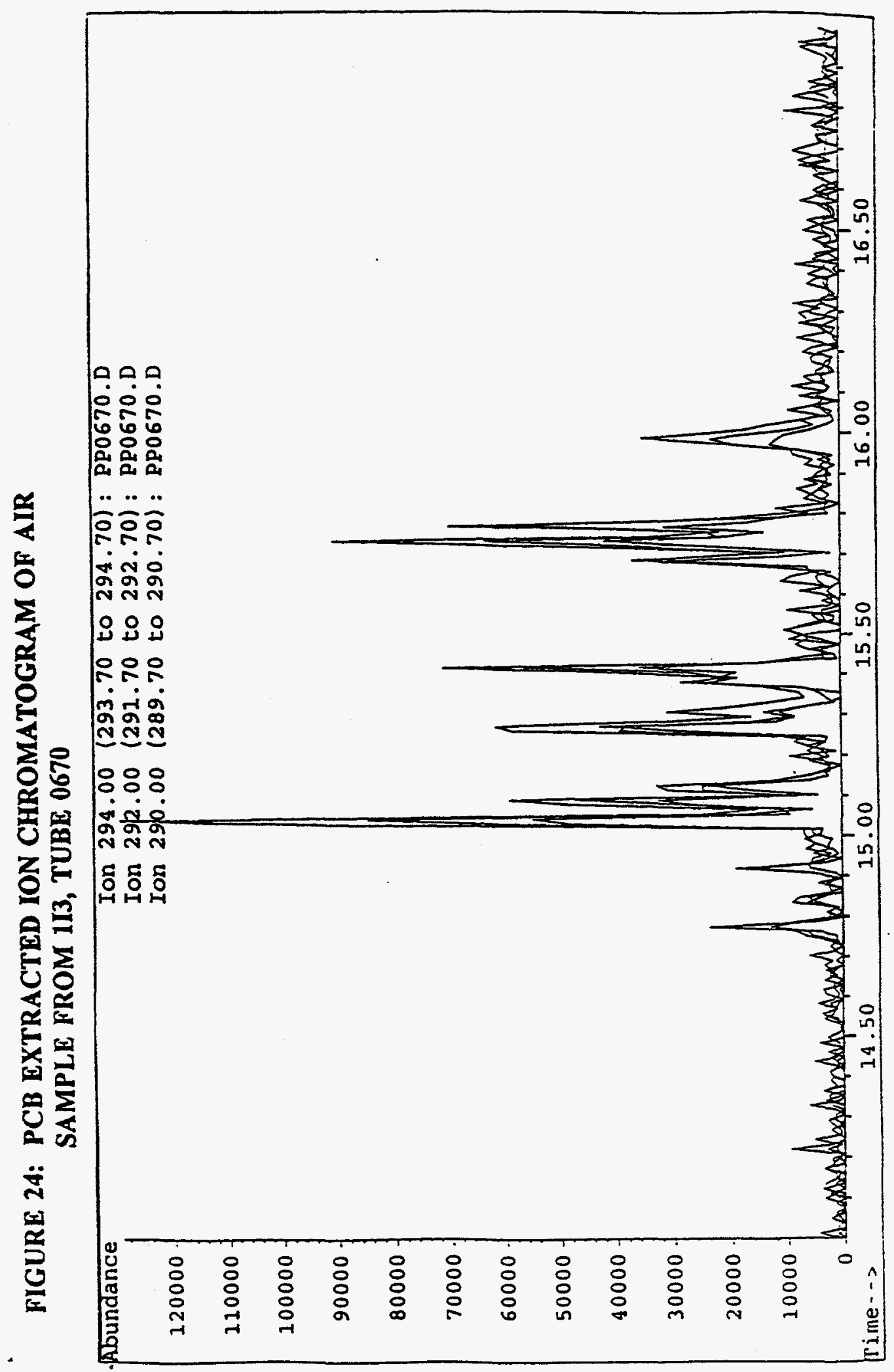




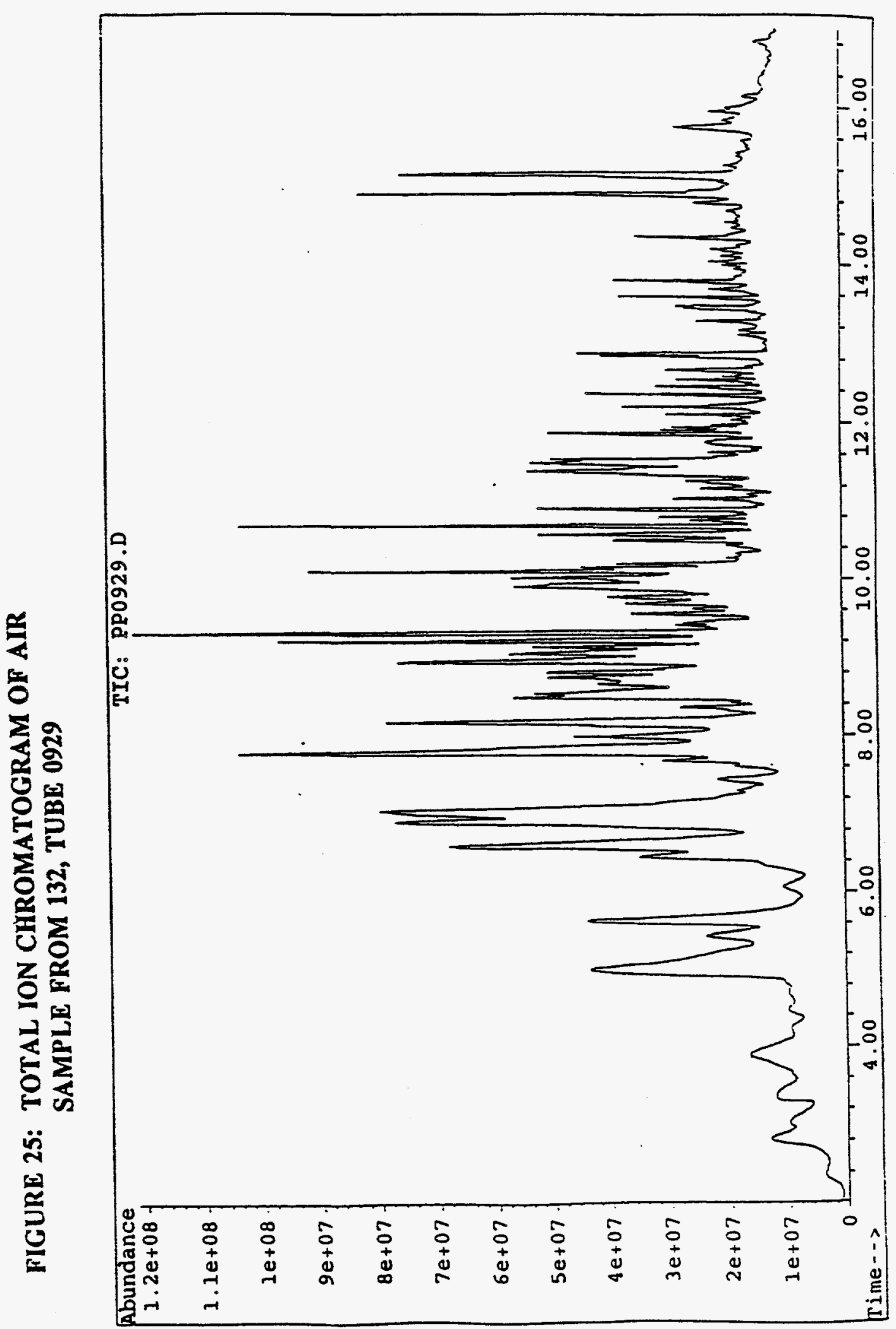




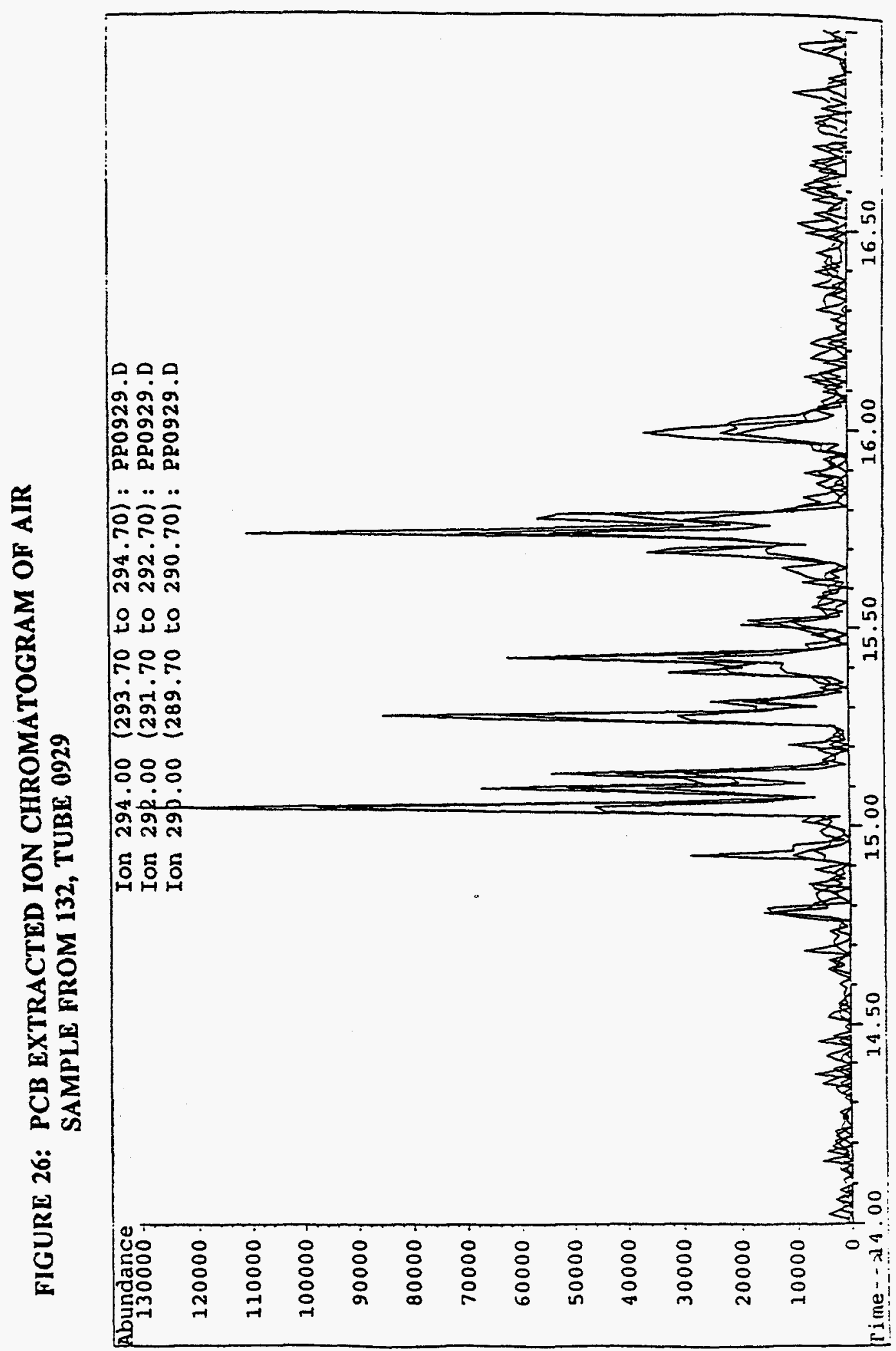


APPENDIX 2:

TABLES 2 THROUGH 14 
TABLE 2. SEMIQUANTITATIVE GC-MS RESULTS OF AIR SAMPLE FROM 3D2, TUBE 0942

\begin{tabular}{|c|c|c|}
\hline $\begin{array}{c}\text { Site 3D2-S, Tube } 0942 \\
\text { Compound }\end{array}$ & $\begin{array}{c}\text { Retention Time } \\
\text { (minutes) }\end{array}$ & $\begin{array}{c}\text { Concentration } \\
\mathrm{pg} / \mathrm{L} \\
\end{array}$ \\
\hline 4-methyl-2-pentanone & not found & not found \\
\hline pentachlorobiphenyl & not found & not found \\
\hline trichlorofluoromethane & 2.32 & 7.9 \\
\hline hexane & 2.82 & 20 \\
\hline diisopropylamine & 3.37 & 0.10 \\
\hline trichloroethylene & 3.90 & 12 \\
\hline toluene & 4.99 & 390 \\
\hline 1-octene & 5.27 & 1.0 \\
\hline 3-heptanone & 5.39 & 82 \\
\hline tetrachloroethylene & 5.61 & 17 \\
\hline dimethyl benzene & 6.90 & 140 \\
\hline propyl benzene & 7.75 & 16 \\
\hline benzaldehyde & 7.82 & 400 \\
\hline dichlorobenzene & 8.34 & 0.70 \\
\hline 2,3,6-trimethyl octane & 8.42 & 1.0 \\
\hline unknown & 8.59 & 0.50 \\
\hline C3 benzene & 8.70 & 10 \\
\hline acetophenone & 8.94 & 230 \\
\hline O,O-diethyl-S-ethylphosphorothioate & 9.40 & 0.0 \\
\hline triethyl ester phosphoric acid & 9.47 & 0.20 \\
\hline decahydro-2-methyl naphthalene & 9.62 & 2.3 \\
\hline unknown & 9.80 & 4.6 \\
\hline benzoic acid & 9.92 & 650 \\
\hline naphthalene & 10.13 & 100 \\
\hline
\end{tabular}


TABLE 2. SEMIQUANTITATIVE GC-MS RESULTS OF AIR SAMPLE FROM 3D2, TUBE 0942

\begin{tabular}{|c|c|c|}
\hline $\begin{array}{c}\text { Site 3D2-S, Tube } 0942 \\
\text { Compound } \\
\end{array}$ & $\begin{array}{c}\text { Retention Time } \\
\text { (minutes) }\end{array}$ & $\begin{array}{c}\text { Concentration } \\
\mathrm{pg} / \mathrm{L} \\
\end{array}$ \\
\hline unknown & 10.13 & 1.4 \\
\hline unknown & 10.35 & 210 \\
\hline unknown & 10.43 & 1.8 \\
\hline benzothiazole & 10.47 & 260 \\
\hline unknown & 10.52 & 0.60 \\
\hline 2-[bis[1-isopropyl] aminoethanol & 10.73 & 0.90 \\
\hline $\mathrm{N}, \mathrm{N}$-dibutylformamide & 10.73 & 0.90 \\
\hline 2-methyl naphthalene & 10.88 & 2.1 \\
\hline irichlorophenol & 11.12 & 0.10 \\
\hline 1-methyl naphthalene & 11.15 & 13 \\
\hline unknown & 11.29 & 1.4 \\
\hline biphenyl & 11.61 & 43 \\
\hline 1,1'-oxybis-benzene & 11.73 & 63 \\
\hline $\mathrm{N}, \mathrm{N}$-diethylbenzamide & 11.92 & 0.10 \\
\hline n-propyl-benzamide & 12.03 & 12 \\
\hline 4-methyl-2,6-di(tert)butyl phenyol & 12.09 & 0.60 \\
\hline C3 naphthalene & 12.15 & 0.60 \\
\hline 1,6-dimethylnaphthalene & 12.19 & 2.1 \\
\hline $\begin{array}{l}\text { 1,5-bis(1,1-dimethylethyl)-3,3-dimethyl- } \\
\text { bicyclo[3.1.0]hexan-2-one }\end{array}$ & 12.22 & 0.30 \\
\hline 1,2-dihydro-acenaphthylene & 12.23 & 0.30 \\
\hline $\begin{array}{l}\text { 2,6-bis(1,1-dimethylethyl)-2,5-cyclohexadiene-1,4- } \\
\text { dione }\end{array}$ & 12.32 & 0.50 \\
\hline 2-methyl-1,1'-biphenyl & 12.32 & 2.1 \\
\hline
\end{tabular}


TABLE 2. SEMIQUANTITATIVE GC-MS RESULTS OF AIR SAMPLE FROM 3D2, TUBE 0942

\begin{tabular}{|c|c|c|}
\hline $\begin{array}{c}\text { Site 3D2-S, Tube } 0942 \\
\text { Compound } \\
\end{array}$ & $\begin{array}{c}\begin{array}{c}\text { Retention Time } \\
\text { (minutes) }\end{array} \\
\end{array}$ & $\begin{array}{c}\text { Concentration } \\
\mathrm{pg} / \mathrm{L} \\
\end{array}$ \\
\hline unknown & 12.64 & 36 \\
\hline unknown & 12.85 & 24 \\
\hline diethyl phthalate & 12.85 & 140 \\
\hline diphenylamine & 12.94 & 0.20 \\
\hline diphenylmethanone & 12.98 & 1.0 \\
\hline unknown & 13.09 & 0.20 \\
\hline isopropyl myristate & 13.77 & 3.1 \\
\hline trichlorobiphenyl & 13.92 & 0.20 \\
\hline 4-hydroxy-3,5-di-tert-butylbenzaldehyde & 14.12 & 0.10 \\
\hline trichlorobiphenyl & 14.17 & 1.1 \\
\hline phenanthrene & 14.34 & 14 \\
\hline dibutyl phthalate & 14.54 & 1.9 \\
\hline trichlorobiphenyl & 14.59 & 0.40 \\
\hline hexadecanoic acid & 14.69 & 2.9 \\
\hline tetrachlorobiphenyl & 14.79 & 0.10 \\
\hline tetrachlorobiphenyl (nc) & 14.94 & 0.30 \\
\hline tetrachlorobiphenyl & 15.14 & 0.30 \\
\hline dioctyl phthalate & 15.30 & 0.10 \\
\hline tetrachlorobiphenyl & 15.43 & 0.40 \\
\hline tetrachlorobiphenyl & 15.46 & 0.0 \\
\hline pentachlorobiphenyl & 15.62 & 0.0 \\
\hline tetrachlorobiphenyl & 15.62 & 0.10 \\
\hline
\end{tabular}


TABLE 3. SEMIQUANTITATIVE GC-MS RESULTS OF AIR SAMPLE FROM 3ET, TUBE 0958

\begin{tabular}{|c|c|c|}
\hline $\begin{array}{c}\text { Site 3ET-S, Tube } 0958 \\
\text { Compound }\end{array}$ & $\begin{array}{l}\text { Retention Time } \\
\text { (minutes) }\end{array}$ & $\begin{array}{c}\text { Concentration } \\
\text { pg/L } \\
\end{array}$ \\
\hline trichlorofluoromethane & 2.14 & 0.40 \\
\hline diisopropylamine & 2.60 & 0.0 \\
\hline hexane & 2.65 & 8.8 \\
\hline trichloroethylene & 2.97 & 0.0 \\
\hline 4-methyl-2-pentanone & 3.69 & 42 \\
\hline toluene & 3.98 & 300 \\
\hline 3-heptanone & 4.20 & 23 \\
\hline 1-octene & 4.22 & 0.30 \\
\hline tetrachloroethylene & 4.35 & 40 \\
\hline dimethyl benzene & 5.14 & 76 \\
\hline benzaldehyde & 5.81 & 110 \\
\hline propyl benzene & 6.07 & 19 \\
\hline 2,3,6-trimethyl octane & 6.35 & 0.70 \\
\hline dichlorobenzene & 6.47 & 1.5 \\
\hline unknown & 6.68 & 1.7 \\
\hline C3 benzene & 6.71 & 57 \\
\hline acetophenone & 6.71 & 57 \\
\hline triethyl ester phosphoric acid & 7.05 & 1.0 \\
\hline unknown & 7.44 & 22 \\
\hline decahydro-2-methyl naphthalene & 7.53 & 0.20 \\
\hline benzoic acid & 7.53 & 140 \\
\hline O,O-diethyl-S-ethylphosphorothioate & 7.69 & 0.0 \\
\hline naphthalene & 7.75 & 5.4 \\
\hline
\end{tabular}


TABLE 3. SEMIQUANTITATIVE GC-MS RESULTS OF AIR SAMPLE FROM 3ET, TUBE 0958

\begin{tabular}{|c|c|c|}
\hline $\begin{array}{l}\text { Site 3ET-S, Tube } 0958 \\
\text { Compound }\end{array}$ & $\begin{array}{l}\text { Retention Time } \\
\text { (minutes) }\end{array}$ & $\begin{array}{c}\text { Concentration } \\
\mathrm{pg} / \mathrm{L}\end{array}$ \\
\hline unknown & 7.80 & 0.50 \\
\hline unknown & 7.93 & 1.0 \\
\hline benzothiazole & 8.12 & 130 \\
\hline unknown & 8.17 & 0.60 \\
\hline unknown & 8.19 & 2.1 \\
\hline unknown & 8.43 & 0.40 \\
\hline 2-methyl naphthalene & 8.51 & 0.80 \\
\hline 2 -[bis[1-isopropyl]aminoethanol & 8.71 & 0.90 \\
\hline N,N-dibutylformamide & 8.71 & 0.90 \\
\hline 1-methyl naphthalene & 8.77 & 6.9 \\
\hline n-propyl-benzamide & 9.02 & 1.0 \\
\hline trichlorophenol & 9.09 & 0.10 \\
\hline $\begin{array}{l}\text { 1,5-bis(1,1-dimethylethyl)-3,3-dimethyl- } \\
\text { bicyclo[3.1.0]hexan-2-one }\end{array}$ & 9.13 & 0.90 \\
\hline biphenyl & 9.21 & 9.2 \\
\hline $\mathrm{N}, \mathrm{N}$-diethylbenzamide & 9.31 & 0.40 \\
\hline $1,1^{\prime}$-oxybis-benzene & 9.33 & 12.0 \\
\hline 1,6-dimethylnaphthalene & 9.42 & 3.8 \\
\hline C3 naphthalene & 9.51 & 1.1 \\
\hline 1,2-dihydro-acenaphthylene & 9.54 & 0.50 \\
\hline 2-methyl-1,1'-biphenyl & 9.60 & 0.10 \\
\hline $\begin{array}{l}\text { 2,6-bis(1,1-dimethylethyl)-2,5-cyclohexadiene- } \\
\text { 1,4-dione }\end{array}$ & 9.60 & 0.60 \\
\hline 4-methyl-2,6-di(tert)butyl phenyol & 9.85 & 0.10 \\
\hline
\end{tabular}


TABLE 3. SEMIQUANTITATIVE GC-MS RESULTS OF AIR SAMPLE FROM 3ET, TUBE 0958

\begin{tabular}{|c|c|c|}
\hline $\begin{array}{c}\text { Site 3ET-S, Tube } 0958 \\
\text { Compound }\end{array}$ & $\begin{array}{c}\text { Retention Time } \\
\text { (minutes) }\end{array}$ & $\begin{array}{c}\text { Concentration } \\
\mathrm{pg} / \mathrm{L}\end{array}$ \\
\hline unknown & 10.00 & 0.30 \\
\hline unknown & 10.03 & 0.50 \\
\hline diphenylamine & 10.06 & 0.40 \\
\hline diphenylmethanone & 10.10 & 0.10 \\
\hline unknown & 10.15 & 0.20 \\
\hline diethyl phthalate & 10.22 & 0.80 \\
\hline trichlorobiphenyl & 10.86 & 0.20 \\
\hline 4-hydroxy-3,5-di-tert-butylbenzaldehyde & 11.03 & 0.50 \\
\hline isopropyl myristate & 11.24 & 0.20 \\
\hline hexadecanoic acid & 11.36 & 0.30 \\
\hline trichlorobiphenyl & 11.37 & 0.30 \\
\hline phenanthrene & 11.43 & 0.30 \\
\hline trichlorobiphenyl & 11.52 & 0.10 \\
\hline tetrachlorobiphenyl & 11.53 & 0.20 \\
\hline tetrachlorobiphenyl (nc) & 11.58 & 0.20 \\
\hline dioctyl phthalate & 11.60 & 22 \\
\hline dibutyl phthalate & 11.64 & 200 \\
\hline tetrachlorobiphenyl & 11.81 & 0.10 \\
\hline tetrachlorobiphenyl & 11.81 & 0.10 \\
\hline tetrachlorobiphenyl (nc) & 12.08 & 0.20 \\
\hline pentachlorobiphenyl & 12.18 & 0.10 \\
\hline tetrachlorobiphenyl & 12.45 & 0.10 \\
\hline diisooctyl phthalate & 12.73 & 0.30 \\
\hline pentachlorobiphenyl & 12.74 & 0.10 \\
\hline
\end{tabular}


TABLE 4. SEMIQUANTITATIVE GC-MS RESULTS OF AIR SAMPLE FROM 4D2, TUBE 0954

\begin{tabular}{|c|c|c|}
\hline $\begin{array}{c}\text { Site 4D2-S, Tube } 0954 \\
\text { Compound }\end{array}$ & $\begin{array}{c}\text { Retention Time } \\
\text { (minutes) }\end{array}$ & $\begin{array}{c}\text { Concentration } \\
\mathrm{pg} / \mathrm{L} \\
\end{array}$ \\
\hline trichlorofluoromethane & 2.13 & 0.10 \\
\hline diisopropylamine & 2.60 & 0.0 \\
\hline hexane & 2.67 & 0.60 \\
\hline trichloroethylene & 3.11 & 0.0 \\
\hline 4-methyl-2-pentanone & 3.69 & 23 \\
\hline toluene & 3.98 & 290 \\
\hline 3-heptanone & 4.20 & 41 \\
\hline 1-octene & 4.22 & 0.20 \\
\hline tetrachloroethylene & 4.34 & 29 \\
\hline dimethyl benzene & 5.14 & 100 \\
\hline benzaldehyde & 5.75 & 4.6 \\
\hline propyl benzene & 6.06 & 30 \\
\hline 2,3,6-trimethyl octane & 6.37 & 0.40 \\
\hline dichlorobenzene & 6.47 & 2.5 \\
\hline C3 benzene & 6.51 & 2.7 \\
\hline unknown & 6.69 & 3.2 \\
\hline acetophenone & 6.83 & 2.6 \\
\hline triethyl ester phosphoric acid & 7.04 & 3.7 \\
\hline decahydro-2-methyl naphthalene & 7.18 & 4.3 \\
\hline unknown & 7.42 & 30 \\
\hline unknown & 7.57 & 3.7 \\
\hline O,O-diethyl-S-ethylphosphorothioate & 7.68 & 0.10 \\
\hline naphthalene & 7.75 & 9.8 \\
\hline benzoic acid & 7.80 & 5.6 \\
\hline
\end{tabular}


TABLE 4. SEMIQUANTITATIVE GC-MS RESULTS OF AIR SAMPLE FROM 4D2, TUBE 0954

\begin{tabular}{|c|c|c|}
\hline $\begin{array}{c}\text { Site 4D2-S, Tube } 0954 \\
\text { Compound } \\
\end{array}$ & $\begin{array}{l}\text { Retention Time } \\
\text { (minutes) }\end{array}$ & $\begin{array}{c}\text { Concentration } \\
\mathrm{pg} / \mathrm{L}\end{array}$ \\
\hline unknown & 7.84 & 0.80 \\
\hline benzothiazole & 8.12 & 77 \\
\hline unknown & 8.19 & 2.9 \\
\hline 2 -[bis[1-isopropyl]aminoethanol & 8.31 & 0.30 \\
\hline $\mathrm{N}, \mathrm{N}$-dibutylformamide & 8.31 & 0.30 \\
\hline unknown & 8.57 & 0.50 \\
\hline trichlorophenol & 8.66 & 0.20 \\
\hline 1-methyl naphthalene & 8.76 & 10 \\
\hline 2-methyl naphthalene & 8.76 & 10 \\
\hline unknown & 8.78 & 0.30 \\
\hline n-propyl-benzamide & 9.34 & 0.40 \\
\hline 1,6-dimethylnaphthalene & 9.12 & 0.40 \\
\hline biphenyl & 9.21 & 17 \\
\hline 1,1'-oxybis-benzene & 9.33 & 24 \\
\hline $\begin{array}{l}\text { 2,6-bis(1,1-dimethylethyl)-2,5- } \\
\text { cyclohexadiene-1,4-dione }\end{array}$ & 9.46 & 10.0 \\
\hline 1,2-dihydro-acenaphthylene & 9.51 & 0.70 \\
\hline $\begin{array}{l}\text { 1,5-bis(1,1-dimethylethyl)-3,3-dimethyl- } \\
\text { bicyclo[3.1.0]hexan-2-one }\end{array}$ & 9.53 & 0.50 \\
\hline 2-methyl-1,1'-biphenyl & 9.59 & 0.10 \\
\hline unknown & 9.65 & 0.20 \\
\hline $\mathrm{N}, \mathrm{N}$-diethylbenzamide & 9.65 & 0.20 \\
\hline 4-methyl-2,6-di(tert)butyl phenyol & 9.85 & 0.50 \\
\hline C3 naphthalene & 9.93 & 0.40 \\
\hline unknown & 10.00 & 0.40 \\
\hline
\end{tabular}


TABLE 4. SEMIQUANTITATIVE GC-MS RESULTS OF AIR SAMPLE FROM 4D2, TUBE 0954

\begin{tabular}{|c|c|c|}
\hline $\begin{array}{c}\text { Site 4D2-S, Tube } 0954 \\
\text { Compound }\end{array}$ & $\begin{array}{c}\text { Retention Time } \\
\text { (minutes) }\end{array}$ & $\begin{array}{c}\text { Concentration } \\
\mathrm{pg} / \mathrm{L} \\
\end{array}$ \\
\hline unknown & 10.19 & 0.30 \\
\hline diphenylmethanone & 10.20 & 0.20 \\
\hline diethyl phthalate & 10.23 & 0.50 \\
\hline diphenylamine & 10.48 & 0.70 \\
\hline 4-hydroxy-3,5-di-tert-butylbenzaldehyde & 10.62 & 0.30 \\
\hline trichlorobiphenyl & 10.98 & 0.10 \\
\hline isopropyl myristate & 11.25 & 0.20 \\
\hline hexadecanoic acid & 11.32 & 0.10 \\
\hline trichlorobiphenyl & 11.32 & 0.10 \\
\hline phenanthrene & 11.40 & 0.30 \\
\hline trichlorobiphenyl & 11.59 & 0.20 \\
\hline dibutyl phthalate & 11.60 & 62 \\
\hline dioctyl phthalate & 11.60 & 7.6 \\
\hline tetrachlorobiphenyl & 11.83 & 0.0 \\
\hline tetrachlorobiphenyl & 11.94 & 0.20 \\
\hline tetrachlorobiphenyl (nc) & 12.00 & 0.10 \\
\hline tetrachlorobiphenyl (nc) & 12.05 & 0.10 \\
\hline tetrachlorobiphenyl & 12.05 & 0.0 \\
\hline tetrachlorobiphenyl & 12.16 & 0.10 \\
\hline pentachlorobiphenyl & 12.60 & 0.20 \\
\hline diisooctyl phthalate & 12.70 & 0.60 \\
\hline pentachlorobiphenyl & 12.74 & 0.10 \\
\hline
\end{tabular}


TABLE 5. SEMIQUANTITATIVE GC-MS RESULTS OF AIR SAMPLE FROM 4FT, TUBE 0936

\begin{tabular}{|c|c|c|}
\hline $\begin{array}{c}\text { Site 4FT-S, Tube } 0936 \\
\text { Compound } \\
\end{array}$ & $\begin{array}{c}\text { Retention Time } \\
\text { (minutes) }\end{array}$ & $\begin{array}{c}\text { Concentration } \\
\mathrm{pg} / \mathrm{L}\end{array}$ \\
\hline trichlorofluoromethane & 2.12 & 0.10 \\
\hline diisopropylamine & 2.51 & 0.0 \\
\hline hexane & 2.68 & 0.30 \\
\hline trichloroethylene & 2.97 & 0.10 \\
\hline 4-methyl-2-pentanone & 3.68 & 93 \\
\hline toluene & 3.97 & 420 \\
\hline 3-heptanone & 4.19 & 29 \\
\hline 1-octene & 4.22 & 0.20 \\
\hline tetrachloroethylene & 4.33 & 38 \\
\hline dimethyl benzene & 5.13 & 110 \\
\hline benzaldehyde & 5.8 & 13 \\
\hline propyl benzene & 6.05 & 32 \\
\hline dichlorobenzene & 6.46 & 3.4 \\
\hline 2,3,6-trimethyl octane & 6.64 & 0.9 \\
\hline unknown & 6.68 & 2.8 \\
\hline C3 benzene & 6.74 & 13 \\
\hline acetophenone & 6.74 & 13 \\
\hline triethyl ester phosphoric acid & 7.03 & 0.80 \\
\hline O,O-diethyl-S-ethylphosphorothioate & 7.35 & 0.0 \\
\hline unknown & 7.42 & 38 \\
\hline decahydro-2-methyl naphthalene & 7.48 & 0.40 \\
\hline unknown & 7.54 & 0.70 \\
\hline naphthalene & 7.74 & 9.4 \\
\hline benzoic acid & 7.77 & 12 \\
\hline
\end{tabular}


TABLE 5. SEMIQUANTITATIVE GC-MS RESULTS OF AIR SAMPLE FROM 4FT, TUBE 0936

\begin{tabular}{|c|c|c|}
\hline $\begin{array}{l}\text { Site 4FT-S, Tube } 0936 \\
\text { Compound }\end{array}$ & $\begin{array}{c}\text { Retention Time } \\
\text { (minutes) }\end{array}$ & $\begin{array}{c}\text { Concentration } \\
\mathrm{pg} / \mathrm{L}\end{array}$ \\
\hline unknown & 7.83 & 0.50 \\
\hline unknown & 7.97 & 59 \\
\hline benzothiazole & 8.11 & 93 \\
\hline unknown & 8.41 & 1.5 \\
\hline unknown & 8.54 & 0.40 \\
\hline 2-[bis[1-isopropyl]aminoethanol & 8.57 & 0.60 \\
\hline $\mathrm{N}, \mathrm{N}$-dibutylformamide & 8.57 & 0.60 \\
\hline 1-methyl naphthalene & 8.75 & 16 \\
\hline 2-methyl naphthalene & 8.75 & 16 \\
\hline trichlorophenol & 9.06 & 0.20 \\
\hline n-propyl-benzamide & 9.07 & 1.8 \\
\hline biphenyl & 9.20 & 17 \\
\hline N,N-diethylbenzamide & 9.30 & 0.10 \\
\hline 1,1'-oxybis-benzene & 9.32 & 19 \\
\hline 4-methyl-2,6-di(tert)butyl phenyol & 9.44 & 0.80 \\
\hline C3 naphthalene & 9.50 & 2.6 \\
\hline 1,6-dimethylnaphthalene & 9.50 & 7.8 \\
\hline $\begin{array}{l}\text { 1,5-bis(1,1-dimethylethyl)-3,3-dimethyl- } \\
\text { bicyclo[3.1.0]hexan-2-one }\end{array}$ & 9.58 & 4.2 \\
\hline 2-methyl-1,1'-biphenyl & 9.59 & 0.30 \\
\hline $\begin{array}{l}\text { 2,6-bis(1,1-dimethylethyl)-2,5-cyclohexadiene- } \\
\text { 1,4-dione }\end{array}$ & 9.62 & 0.90 \\
\hline 1,2-dihydro-acenaphthylene & 9.82 & 0.30 \\
\hline unknown & 9.99 & 1.0 \\
\hline unknown & 10.02 & 0.20 \\
\hline
\end{tabular}


TABLE 5. SEMIQUANTITATIVE GC-MS RESULTS OF AIR SAMPLE FROM 4FT, TUBE 0936

\begin{tabular}{|c|c|c|}
\hline $\begin{array}{c}\text { Site 4FT-S, Tube } 0936 \\
\text { Compound }\end{array}$ & $\begin{array}{c}\text { Retention Time } \\
\text { (minutes) }\end{array}$ & $\begin{array}{c}\text { Concentration } \\
\text { pg/L } \\
\end{array}$ \\
\hline diphenylamine & 10.08 & 0.60 \\
\hline unknown & 10.14 & 0.10 \\
\hline diphenylmethanone & 10.14 & 0.40 \\
\hline diethyl phthalate & 10.23 & 0.20 \\
\hline 4-hydroxy-3,5-di-tert-butylbenzaldehyde & 10.65 & 0.10 \\
\hline trichlorobiphenyl & 11.12 & 0.10 \\
\hline isopropyl myristate & 11.20 & 0.20 \\
\hline trichlorobiphenyl & 11.32 & 0.10 \\
\hline trichlorobiphenyl & 11.36 & 0.20 \\
\hline dioctyl phthalate & 11.49 & 0.30 \\
\hline tetrachlorobiphenyl & 11.55 & 0.10 \\
\hline dibutyl phthalate & 11.55 & 76 \\
\hline tetrachlorobiphenyl (nc) & 11.57 & 0.40 \\
\hline phenanthrene & 11.68 & 2.6 \\
\hline hexadecanoic acid & 11.76 & 0.40 \\
\hline tetrachlorobiphenyl (nc) & 12.06 & 0.10 \\
\hline tetrachlorobiphenyl & 12.09 & 0.0 \\
\hline tetrachlorobiphenyl & 12.23 & 0.0 \\
\hline tetrachlorobiphenyl & 12.23 & 0.0 \\
\hline pentachlorobiphenyl & 12.60 & 0.10 \\
\hline diisooctylphthalate & 12.73 & 0.70 \\
\hline pentachlorobiphenyl & 12.74 & 0.10 \\
\hline
\end{tabular}


TABLE 6. SEMIQUANTITATIVE GC-MS RESULTS OF AIR SAMPLE FROM 4C, TUBE 0714

\begin{tabular}{|l|c|c|}
\hline \multicolumn{1}{|c|}{$\begin{array}{c}\text { Site 4C, Tube 0714 } \\
\text { Compound }\end{array}$} & $\begin{array}{c}\text { Retention Time } \\
\text { (minutes) }\end{array}$ & $\begin{array}{c}\text { Concentration } \\
\text { pg/L }\end{array}$ \\
\hline \hline 2-[bis[1-isopropyl]aminoethanol & not found & not found \\
\hline trichlorofluoromethane & 2.15 & 1.9 \\
\hline diisopropylamine & 2.51 & 0.10 \\
\hline n-(isopropyl)-2-propanamine & 2.62 & 31 \\
\hline hexane & 2.62 & 24 \\
\hline chloroform & 2.67 & 0.10 \\
\hline trichloroethylene & 2.95 & 0.0 \\
\hline 4-methyl-2-pentanone & 3.67 & 30 \\
\hline toluene & 3.97 & 310 \\
\hline 1-octene & 4.11 & 3.0 \\
\hline 3-heptanone & 4.18 & 30.0 \\
\hline tetrachloroethylene & 4.33 & 95 \\
\hline dimethyl benzene & 5.13 & 140 \\
\hline 1,4-oxathiane & 5.68 & 0.70 \\
\hline benzaldehyde & 5.79 & 140 \\
\hline propyl benzene & 5.91 & 7.0 \\
\hline phenol & 6.27 & 0.20 \\
\hline 2,3,6-trimethyl octane & 6.34 & 0.30 \\
\hline dichlorobenzene & 6.45 & 2.1 \\
\hline acetophenone & 6.50 & 3.2 \\
\hline unknown & 6.67 & 2.7 \\
\hline limonene & 6.92 & 1.4 \\
\hline decahydro-2-methyl naphthalene & 7.00 & 0.20 \\
\hline
\end{tabular}


TABLE 6. SEMIQUANTITATIVE GC-MS RESULTS OF AIR SAMPLE FROM 4C, TUBE 0714

\begin{tabular}{|c|c|c|}
\hline $\begin{array}{c}\text { Site } 4 C, \text { Tube } 0714 \\
\text { Compound } \\
\end{array}$ & $\begin{array}{l}\text { Retention Time } \\
\text { (minutes) }\end{array}$ & $\begin{array}{c}\text { Concentration } \\
\mathrm{pg} / \mathrm{L} \\
\end{array}$ \\
\hline triethyl ester phosphoric acid & 7.03 & 4.1 \\
\hline C3 benzene & 7.14 & 1.5 \\
\hline benzoic acid & 7.53 & 380 \\
\hline unknown & 7.69 & 4.3 \\
\hline unknown & 7.70 & 1.4 \\
\hline naphthalene & 7.77 & 79 \\
\hline unknown & 7.77 & 0.80 \\
\hline O,O-diethyl-S-ethylphosphorothioate & 7.89 & 0.10 \\
\hline benzothiazole & 8.10 & 38 \\
\hline trichlorobenzene & 8.17 & 0.10 \\
\hline unknown & 8.17 & 1.6 \\
\hline unknown & 8.45 & 0.70 \\
\hline N,N-dibutylformamide & 8.50 & 12 \\
\hline 1-methyl naphthalene & 8.62 & 28 \\
\hline unknown & 8.93 & 0.30 \\
\hline trichlorophenol & 9.22 & 0.10 \\
\hline 2-methyl naphthalene & 9.30 & 12 \\
\hline 1,1'-oxybis-benzene & 9.30 & 35 \\
\hline $\begin{array}{l}\text { 2,6-bis(1,1-dimethylethyl)-2,5-cyclohexadiene- } \\
\text { 1,4-dione }\end{array}$ & 9.39 & 0.50 \\
\hline biphenyl & 9.40 & 2.1 \\
\hline 1,6-dimethylnaphthalene & 9.64 & 6.9 \\
\hline $\begin{array}{l}\text { 1,5-bis(1,1-dimethylethyl)-3,3-dimethyl- } \\
\text { bicyclo[3.1.0]hexan-2-one }\end{array}$ & 9.67 & 0.30 \\
\hline
\end{tabular}


TABLE 6. SEMIQUANTITATIVE GC-MS RESULTS OF AIR SAMPLE FROM 4C, TUBE 0714

\begin{tabular}{|c|c|c|}
\hline $\begin{array}{c}\text { Site } 4 C, \text { Tube } 0714 \\
\text { Compound }\end{array}$ & $\begin{array}{c}\text { Retention Time } \\
\text { (minutes) }\end{array}$ & $\begin{array}{c}\text { Concentration } \\
\text { pg/L }\end{array}$ \\
\hline diethyl phthalate & 9.81 & 0.40 \\
\hline 2-methyl-1,1'-biphenyl & 9.88 & 2.9 \\
\hline 4-methyl-2,6-di(tert)butyl phenyol & 10.03 & 0.20 \\
\hline 1,2-dihydro-acenaphthylene & 10.04 & 2.7 \\
\hline n-propyl-benzamide & 10.10 & 8.3 \\
\hline unknown & 10.10 & 8.3 \\
\hline diphenylamine & 10.22 & 1.5 \\
\hline $\mathrm{N}, \mathrm{N}$-diethylbenzamide & 10.23 & 0.50 \\
\hline unknown & 10.28 & 0.20 \\
\hline C3 naphthalene & 10.44 & 5.2 \\
\hline unknown & 10.62 & 1.2 \\
\hline diphenylmethanone & 10.68 & 0.80 \\
\hline trichlorobiphenyl & 10.82 & 0.10 \\
\hline isopropyl myristate & 11.26 & 0.30 \\
\hline 4-hydroxy-3,5-di-tert-butylbenzaldehyde & 11.45 & 0.20 \\
\hline trichlorobiphenyl & 11.47 & 0.50 \\
\hline trichlorobiphenyl & 11.47 & 0.50 \\
\hline dibutyl phthalate & 11.57 & 160 \\
\hline tetrachlorobiphenyl (nc) & 11.64 & 0.20 \\
\hline tetrachlorobiphenyl & 11.64 & 0.20 \\
\hline tetrachlorobiphenyl & 11.72 & 0.10 \\
\hline phenanthrene & 11.88 & 36 \\
\hline tetrachlorobiphenyl (nc) & 11.95 & 0.10 \\
\hline
\end{tabular}


106

TABLE 6. SEMIQUANTITATIVE GC-MS RESULTS OF AIR SAMPLE FROM 4C, TUBE 0714

\begin{tabular}{|l|c|c|}
\hline \multicolumn{1}{|c|}{$\begin{array}{c}\text { Site 4C, Tube 0714 } \\
\text { Compound }\end{array}$} & $\begin{array}{c}\text { Retention Time } \\
\text { (minutes) }\end{array}$ & $\begin{array}{c}\text { Concentration } \\
\text { pg/L }\end{array}$ \\
\hline \hline dioctyl phthalate & 12.05 & 0.20 \\
\hline pentachlorobiphenyl & 12.23 & 0.10 \\
\hline hexadecanoic acid & 12.56 & 27 \\
\hline tetrachlorobiphenyl & 12.74 & 6.7 \\
\hline diisooctyl phthalate & 12.93 & 3.0 \\
\hline tetrachlorobiphenyl & 13.05 & 4.8 \\
\hline pentachlorobiphenyl & 13.20 & 0.10 \\
\hline
\end{tabular}


TABLE 7. SEMIQUANTITATIVE GC-MS RESULTS OF AIR SAMPLE FROM 4A, TUBE 0959

\begin{tabular}{|c|c|c|}
\hline $\begin{array}{c}\text { Site 4A-S, Tube } 0959 \\
\text { Compound }\end{array}$ & $\begin{array}{c}\text { Retention Time } \\
\text { (minutes) }\end{array}$ & $\begin{array}{c}\text { Concentration } \\
\mathrm{pg} / \mathrm{L} \\
\end{array}$ \\
\hline trichlorofluoromethane & 2.37 & 78 \\
\hline hexane & 2.82 & 120 \\
\hline diisopropylamine & 3.23 & 0.60 \\
\hline trichloroethylene & 3.92 & 44 \\
\hline 4-methyl-2-pentanone & 4.42 & 6.9 \\
\hline toluene & 5.00 & 610 \\
\hline 1-octene & 5.06 & 0.20 \\
\hline 3-heptanone & 5.44 & 43 \\
\hline tetrachloroethylene & 5.64 & 130 \\
\hline dimethyl benzene & 6.91 & 180 \\
\hline propyl benzene & 7.76 & 23 \\
\hline benzaldehyde & 7.83 & 240 \\
\hline 2,3,6-trimethyl octane & 8.12 & 3.3 \\
\hline dichlorobenzene & 8.43 & 4.0 \\
\hline unknown & 8.59 & 0.50 \\
\hline C3 benzene & 8.71 & 5.8 \\
\hline acetophenone & 8.93 & 150 \\
\hline triethyl ester phosphoric acid & 9.03 & 0.70 \\
\hline decahydro-2-methyl naphthalene & 9.25 & 0.20 \\
\hline unknown & 9.51 & 4.1 \\
\hline unknown & 9.70 & 2.9 \\
\hline O,O-diethyl-S-ethylphosphorothioate & 9.86 & 0.10 \\
\hline benzoic acid & 9.88 & 480 \\
\hline
\end{tabular}


TABLE 7. SEMIQUANTITATIVE GC-MS RESULTS OF AIR SAMPLE FROM 4A, TUBE 0959

\begin{tabular}{|c|c|c|}
\hline $\begin{array}{l}\text { Site 4A-S, Tube } 0959 \\
\text { Compound }\end{array}$ & $\begin{array}{l}\text { Retention Time } \\
\text { (minutes) }\end{array}$ & $\begin{array}{c}\text { Concentration } \\
\text { pg/L }\end{array}$ \\
\hline naphthalene & 10.14 & 88 \\
\hline unknown & 10.38 & 42 \\
\hline unknown & 10.44 & 1.4 \\
\hline benzothiazole & 10.49 & 150 \\
\hline 2-[bis[1-isopropyl]aminoethanol & 10.67 & 0.50 \\
\hline $\mathrm{N}, \mathrm{N}$-dibutylformamide & 10.67 & 0.50 \\
\hline unknown & 10.80 & 1.0 \\
\hline unknown & 10.88 & 0.90 \\
\hline 2-methyl naphthalene & 10.89 & 1.1 \\
\hline 1-methyl naphthalene & 11.16 & 13 \\
\hline trichlorophenol & 11.56 & 0.20 \\
\hline 1,6-dimethylnaphthalene & 11.62 & 0.40 \\
\hline 1,1'-oxybis-benzene & 11.74 & 17 \\
\hline biphenyl & 11.84 & 0.80 \\
\hline n-propyl-benzamide & 12.12 & 3.7 \\
\hline C3 naphthalene & 12.16 & 0.70 \\
\hline unknown & 12.23 & 2.1 \\
\hline 2-methyl-1,1'-biphenyl & 12.27 & 0.1 \\
\hline $\begin{array}{l}\text { 1,5-bis(1,1-dimethylethyl)-3,3-dimethyl- } \\
\text { bicyclo[3.1.0]hexan-2-one }\end{array}$ & 12.28 & 0.200 \\
\hline $\begin{array}{l}\text { 2,6-bis(1,1-dimethylethyl)-2,5-cyclohexadiene-1,4- } \\
\text { dione }\end{array}$ & 12.32 & 0.60 \\
\hline $\mathrm{N}, \mathrm{N}$-diethylbenzamide & 12.38 & 0.30 \\
\hline 1,2-dihydro-acenaphthylene & 12.50 & 1.4 \\
\hline
\end{tabular}


TABLE 7. SEMIQUANTITATIVE GC-MS RESULTS OF AIR SAMPLE FROM 4A, TUBE 0959

\begin{tabular}{|c|c|c|}
\hline $\begin{array}{c}\text { Site 4A-S, Tube } 0959 \\
\text { Compound }\end{array}$ & $\begin{array}{c}\text { Retention Time } \\
\text { (minutes) }\end{array}$ & $\begin{array}{c}\text { Concentration } \\
\mathrm{pg} / \mathrm{L}\end{array}$ \\
\hline 4-methyl-2,6-di(tert)butyl phenyol & 12.61 & 0.20 \\
\hline unknown & 12.65 & 20.0 \\
\hline diethyl phthalate & 12.86 & 41 \\
\hline diphenylamine & 12.93 & 0.20 \\
\hline diphenylmethanone & 12.99 & 0.60 \\
\hline unknown & 13.07 & 0.50 \\
\hline 4-hydroxy-3,5-di-tert-butylbenzaldehyde & 13.64 & 0.10 \\
\hline isopropyl myristate & 13.77 & 2.4 \\
\hline trichlorobiphenyl & 13.87 & 0.10 \\
\hline trichlorobiphenyl & 14.17 & 1.3 \\
\hline phenanthrene & 14.35 & 26 \\
\hline dibutyl phthalate & 14.55 & 1.9 \\
\hline trichlorobiphenyl & 14.60 & 0.50 \\
\hline tetrachlorobiphenyl (nc) & 14.84 & 0.10 \\
\hline dioctyl phthalate & 14.87 & 0.0 \\
\hline tetrachlorobiphenyl & 15.05 & 1.2 \\
\hline hexadecanoic acid & 15.11 & 0.70 \\
\hline tetrachlorobiphenyl & 15.31 & 0.30 \\
\hline tetrachlorobiphenyl & 15.40 & 0.20 \\
\hline tetrachlorobiphenyl (nc) & 15.42 & 0.90 \\
\hline tetrachlorobiphenyl & 15.70 & 0.40 \\
\hline diisooctyl phthalate & 16.20 & 350 \\
\hline pentachlorobiphenyl & 16.29 & 0.10 \\
\hline pentachlorobiphenyl & 16.32 & 0.30 \\
\hline
\end{tabular}


TABLE 8. SEMIQUANTITATIVE GC-MS RESULTS OF AIR SAMPLE FROM 1FA, TUBE 0824

\begin{tabular}{|c|c|c|}
\hline $\begin{array}{c}\text { Site } 1 \mathrm{~F}-\mathrm{A}, \text { Tube } 0824 \\
\text { Compound }\end{array}$ & $\begin{array}{l}\text { Retention Time } \\
\text { (minutes) }\end{array}$ & $\begin{array}{c}\text { Concentration } \\
\mathrm{pg} / \mathrm{L}\end{array}$ \\
\hline 4-methyl-2-pentanone & not found & not found \\
\hline unknown & not found & not found \\
\hline trichlorofluoromethane & 2.36 & 29 \\
\hline hexane & 2.81 & 16 \\
\hline diisopropylamine & 3.22 & 0.30 \\
\hline trichloroethylene & 3.96 & 55 \\
\hline toluene & 5.02 & 1200 \\
\hline 1-octene & 5.28 & 3.0 \\
\hline 3-heptanone & 5.42 & 120 \\
\hline tetrachloroethylene & 5.64 & 170 \\
\hline dimethyl benzene & 6.91 & 140 \\
\hline propyl benzene & 7.76 & 40.0 \\
\hline benzaldehyde & 7.83 & 340 \\
\hline 2,3,6-trimethyl octane & 8.12 & 3.9 \\
\hline dichlorobenzene & 8.43 & 10.0 \\
\hline C3 benzene & 8.71 & 16 \\
\hline acetophenone & 8.71 & 16 \\
\hline unknown & 8.91 & 6.1 \\
\hline triethyl ester phosphoric acid & 9.09 & 0.50 \\
\hline decahydro-2-methyl naphthalene & 9.21 & 0.30 \\
\hline O,O-diethyl-S-ethylphosphorothioate & 9.40 & 0.0 \\
\hline benzoic acid & 9.69 & 9.3 \\
\hline unknown & 9.75 & 58 \\
\hline unknown & 10.10 & 17 \\
\hline
\end{tabular}


TABLE 8. SEMIQUANTITATIVE GC-MS RESULTS OF AIR SAMPLE FROM IFA, TUBE 0824

\begin{tabular}{|c|c|c|}
\hline $\begin{array}{l}\text { Site 1F-A, Tube } 0824 \\
\text { Compound }\end{array}$ & $\begin{array}{l}\text { Retention Time } \\
\text { (minutes) }\end{array}$ & $\begin{array}{c}\text { Concentration } \\
\mathrm{pg} / \mathrm{L}\end{array}$ \\
\hline unknown & 10.10 & 6.8 \\
\hline naphthalene & 10.14 & 150 \\
\hline benzothiazole & 10.49 & 96 \\
\hline unknown & 10.81 & 2.5 \\
\hline 2-methyl naphthalene & 10.89 & 2.8 \\
\hline 2-[bis[1-isopropyl] aminoethanol & 10.90 & 42 \\
\hline N,N-dibutylformamide & 10.90 & 42 \\
\hline unknown & 10.96 & 9.0 \\
\hline trichlorophenol & 11.11 & 0.20 \\
\hline 1-methyl naphthalene & 11.16 & 20.0 \\
\hline biphenyl & 11.62 & 85 \\
\hline 1,6-dimethylnaphthalene & 11.62 & 0.80 \\
\hline $\begin{array}{l}\text { 1,5-bis(1,1-dimethylethyl)-3,3-dimethyl- } \\
\text { bicyclo[3.1.0]hexan-2-one }\end{array}$ & 11.71 & 0.50 \\
\hline $1,1^{\prime}$-oxybis-benzene & 11.74 & 110 \\
\hline N,N-diethylbenzamide & 11.93 & 0.20 \\
\hline 4-methyl-2,6-di(tert)butyl phenyol & 12.01 & 0.60 \\
\hline n-propyl-benzamide & 12.03 & 13 \\
\hline C3 naphthalene & 12.16 & 0.60 \\
\hline unknown & 12.23 & 22 \\
\hline $\begin{array}{l}\text { 2,6-bis(1,1-dimethylethyl)-2,5-cyclohexadiene- } \\
\text { 1,4-dione }\end{array}$ & 12.32 & 1.0 \\
\hline 2-methyl-1, l'-biphenyl & 12.33 & 3.3 \\
\hline unknown & 12.53 & 0.60 \\
\hline 1,2-dihydro-acenaphthylene & 12.62 & 1.0 \\
\hline
\end{tabular}


TABLE 8. SEMIQUANTITATIVE GC-MS RESULTS OF AIR SAMPLE FROM IFA, TUBE 0824

\begin{tabular}{|c|c|c|}
\hline $\begin{array}{c}\text { Site } 1 F-A, \text { Tube } 0824 \\
\text { Compound }\end{array}$ & $\begin{array}{c}\text { Retention Time } \\
\text { (minutes) }\end{array}$ & $\begin{array}{c}\text { Concentration } \\
\mathrm{pg} / \mathrm{L} \\
\end{array}$ \\
\hline unknown & 12.64 & 72 \\
\hline diethyl phthalate & 12.86 & 70 \\
\hline diphenylamine & 12.87 & 1.1 \\
\hline diphenylmethanone & 12.99 & 1.1 \\
\hline 4-hydroxy-3,5-di-tert-butylbenzaldehyde & 13.61 & 0.10 \\
\hline isopropyl myristate & 13.78 & 4.3 \\
\hline trichlorobiphenyl & 14.18 & 8.8 \\
\hline phenanthrene & 14.35 & 59 \\
\hline trichlorobiphenyl & 14.40 & 4.0 \\
\hline trichlorobiphenyl & 14.60 & 1.1 \\
\hline tetrachlorobiphenyl & 14.79 & 1.4 \\
\hline hexadecanoic acid & 15.05 & 2.0 \\
\hline dibutyl phthalate & 15.12 & 72 \\
\hline dioctyl phthalate & 15.13 & 21 \\
\hline tetrachlorobiphenyl & 15.32 & 2.0 \\
\hline tetrachlorobiphenyl & 15.40 & 2.8 \\
\hline tetrachlorobiphenyl (nc) & 15.52 & 1.5 \\
\hline tetrachlorobiphenyl & 15.75 & 8.2 \\
\hline tetrachlorobiphenyl & 15.75 & 8.2 \\
\hline pentachlorobiphenyl & 16.33 & 0.80 \\
\hline pentachlorobiphenyl & 16.71 & 0.30 \\
\hline
\end{tabular}


TABLE 9. SEMIQUANTITATIVE GC-MS RESULTS OF AIR SAMPLE FROM 1C2, TUBE 0963

\begin{tabular}{|c|c|c|}
\hline $\begin{array}{c}\text { Site } 1 \text { C-2, Tube } 0963 \\
\text { Compound }\end{array}$ & $\begin{array}{c}\text { Retention Time } \\
\text { (minutes) }\end{array}$ & $\begin{array}{c}\text { Concentration } \\
\mathrm{pg} / \mathrm{L} \\
\end{array}$ \\
\hline trichlorofluoromethane & 2.32 & 0.10 \\
\hline hexane & 2.81 & 0.0 \\
\hline diisopropylamine & 3.34 & 4.6 \\
\hline trichloroethylene & 3.91 & 26 \\
\hline 4-methyl-2-pentanone & 4.44 & 5.1 \\
\hline toluene & 4.97 & 1500 \\
\hline 1-octene & 5.31 & 1.7 \\
\hline 3-heptanone & 5.42 & 76 \\
\hline tetrachloroethylene & 5.63 & 480 \\
\hline dimethyl benzene & 6.91 & 530 \\
\hline propyl benzene & 7.75 & 64 \\
\hline benzaldehyde & 7.83 & 320 \\
\hline 2,3,6-trimethyl octane & 8.12 & 14 \\
\hline dichlorobenzene & 8.43 & 22 \\
\hline C3 benzene & 8.47 & 160 \\
\hline unknown & 8.60 & 3.3 \\
\hline acetophenone & 8.69 & 13 \\
\hline triethyl ester phosphoric acid & 9.37 & 15 \\
\hline decahydro-2-methyl naphthalene & 9.62 & 8.8 \\
\hline O,O-diethyl-S-ethylphosphorothioate & 9.68 & 24 \\
\hline unknown & 9.91 & 24 \\
\hline benzoic acid & 9.92 & 180 \\
\hline unknown & 10.09 & 14 \\
\hline unknown & 10.10 & 5.5 \\
\hline
\end{tabular}


TABLE 9. SEMIQUANTITATIVE GC-MS RESULTS OF AIR SAMPLE FROM 1C2, TUBE 0963

\begin{tabular}{|c|c|c|}
\hline $\begin{array}{l}\text { Site 1C-2, Tube } 0963 \\
\text { Compound }\end{array}$ & $\begin{array}{l}\text { Retention Time } \\
\text { (minutes) }\end{array}$ & $\begin{array}{c}\text { Concentration } \\
\mathrm{pg} / \mathrm{L}\end{array}$ \\
\hline naphthalene & 10.13 & 200 \\
\hline unknown & 10.18 & 87 \\
\hline benzothiazole & 10.48 & 140 \\
\hline 2-methyl naphthalene & 10.89 & 8.7 \\
\hline $\mathrm{N}, \mathrm{N}$-dibutylformamide & 10.89 & 160 \\
\hline 2-[bis[1-isopropyl] aminoethanol & 10.89 & 160 \\
\hline unknown & 10.94 & 4.6 \\
\hline 1-methyl naphthalene & 11.16 & 54 \\
\hline unknown & 11.29 & 15 \\
\hline 1,6-dimethylnaphthalene & 11.60 & 1.4 \\
\hline biphenyl & 11.61 & 37 \\
\hline trichlorophenol & 11.65 & 0.60 \\
\hline n-propyl-benzamide & 11.71 & 140 \\
\hline $1,1^{\prime}$-oxybis-benzene & 11.73 & 19 \\
\hline $\begin{array}{l}\text { 1,5-bis(1,1-dimethylethyl)-3,3-dimethyl- } \\
\text { bicyclo[3.1.0]hexan-2-one }\end{array}$ & 11.84 & 1.2 \\
\hline C3 naphthalene & 12.14 & 1.3 \\
\hline $\begin{array}{l}\text { 2,6-bis(1,1-dimethylethyl)-2,5-cyclohexadiene-1,4- } \\
\text { dione }\end{array}$ & 12.31 & 1.3 \\
\hline 2-methyl-1,1'-biphenyl & 12.32 & 11 \\
\hline N,N-diethylbenzamide & 12.43 & 1.2 \\
\hline unknown & 12.44 & 49 \\
\hline 4-methyl-2,6-di(tert)butyl phenyol & 12.58 & 0.90 \\
\hline 1,2-dihydro-acenaphthylene & 12.61 & 2.2 \\
\hline unknown & 12.84 & 13 \\
\hline
\end{tabular}


TABLE 9. SEMIQUANTITATIVE GC-MS RESULTS OF AIR SAMPLE FROM 1C2, TUBE 0963

\begin{tabular}{|c|c|c|}
\hline $\begin{array}{c}\text { Site 1C-2, Tube } 0963 \\
\text { Compound }\end{array}$ & $\begin{array}{c}\text { Retention Time } \\
\text { (minutes) }\end{array}$ & $\begin{array}{c}\text { Concentration } \\
\mathrm{pg} / \mathrm{L} \\
\end{array}$ \\
\hline diethyl phthalate & 12.85 & 100 \\
\hline diphenylmethanone & 12.97 & 2.4 \\
\hline unknown & 13.04 & 0.60 \\
\hline diphenylamine & 13.40 & 3.5 \\
\hline 4-hydroxy-3,5-di-tert-butylbenzaldehyde & 13.68 & 0.20 \\
\hline isopropyl myristate & 13.76 & 4.4 \\
\hline trichlorobiphenyl & 13.92 & 0.20 \\
\hline phenanthrene & 14.33 & 98 \\
\hline dibutyl phthalate & 14.55 & 5.3 \\
\hline trichlorobiphenyl & 14.58 & 0.50 \\
\hline tetrachlorobiphenyl & 14.78 & 0.40 \\
\hline trichlorobiphenyl & 14.89 & 1.2 \\
\hline tetrachlorobiphenyl (nc) & 14.92 & 0.40 \\
\hline tetrachlorobiphenyl & 15.03 & 2.3 \\
\hline hexadecanoic acid & 15.15 & 0.70 \\
\hline dioctyl phthalate & 15.18 & 28 \\
\hline tetrachlorobiphenyl (nc) & 15.42 & 2.8 \\
\hline tetrachlorobiphenyl & 15.73 & 2.3 \\
\hline tetrachlorobiphenyl & 16.07 & 0.20 \\
\hline diisooctyl phthalate & 16.29 & 1.3 \\
\hline pentachlorobiphenyl & 16.31 & 0.20 \\
\hline pentachlorobiphenyl & 16.31 & 0.30 \\
\hline
\end{tabular}


TABLE 10. SEMIQUANTITATIVE GC-MS RESULTS OF AIR SAMPLE FROM 1D1, TUBE 0953

\begin{tabular}{|c|c|c|}
\hline $\begin{array}{c}\text { Site } 1 \text { D-1, Tube } 0953 \\
\text { Compound }\end{array}$ & $\begin{array}{c}\text { Retention Time } \\
\text { (minutes) }\end{array}$ & $\begin{array}{c}\text { Concentration } \\
\mathrm{pg} / \mathrm{L}\end{array}$ \\
\hline trichlorofluoromethane & 2.43 & 0.30 \\
\hline hexane & 2.79 & 4.5 \\
\hline diisopropylamine & 3.34 & 11 \\
\hline trichloroethylene & 3.89 & 20 \\
\hline 4-methyl-2-pentanone & 4.44 & 5.0 \\
\hline toluene & 4.99 & 1000 \\
\hline 1-octene & 5.31 & 0.30 \\
\hline 3-heptanone & 5.42 & 54 \\
\hline tetrachloroethylene & 5.61 & 180 \\
\hline dimethyl benzene & 6.90 & 430 \\
\hline benzaldehyde & 7.81 & 370 \\
\hline propyl benzene & 7.84 & 110 \\
\hline dichlorobenzene & 8.42 & 13 \\
\hline $\mathrm{C} 3$ benzene & 8.45 & 85 \\
\hline 2,3,6-trimethyl octane & 8.48 & 1.0 \\
\hline unknown & 8.89 & 7.0 \\
\hline acetophenone & 9.02 & 38 \\
\hline triethyl ester phosphoric acid & 9.31 & 160 \\
\hline O,O-diethyl-S-ethylphosphorothioate & 9.65 & 12 \\
\hline decahydro-2-methyl naphthalene & 9.67 & 0.9 \\
\hline unknown & 9.89 & 14 \\
\hline benzoic acid & 9.91 & 600 \\
\hline unknown & 10.07 & 12 \\
\hline naphthalene & 10.07 & 16 \\
\hline
\end{tabular}


TABLE 10. SEMIQUANTITATIVE GC-MS RESULTS OF AIR SAMPLE FROM 1D1, TUBE 0953

\begin{tabular}{|c|c|c|}
\hline $\begin{array}{l}\text { Site 1D-1, Tube } 0953 \\
\text { Compound } \\
\end{array}$ & $\begin{array}{c}\text { Retention Time } \\
\text { (minutes) }\end{array}$ & $\begin{array}{c}\text { Concentration } \\
\mathrm{pg} / \mathrm{L}\end{array}$ \\
\hline unknown & 10.17 & 50 \\
\hline benzothiazole & 10.46 & 220 \\
\hline unknown & 10.60 & 1.4 \\
\hline 2-methyl naphthalene & 10.87 & 5.2 \\
\hline $\mathrm{N}, \mathrm{N}$-dibutylformamide & 10.88 & 140 \\
\hline 2 -[bis[1-isopropyl]aminoethanol & 10.88 & 140 \\
\hline unknown & 10.90 & 1.5 \\
\hline 1-methyl naphthalene & 11.14 & 37 \\
\hline trichlorophenol & 11.18 & 0.40 \\
\hline unknown & 11.27 & 11 \\
\hline 1,6-dimethylnaphthalene & 11.60 & 1.2 \\
\hline 1,1'-oxybis-benzene & 11.72 & 9.8 \\
\hline biphenyl & 11.82 & 1.9 \\
\hline $\begin{array}{l}\text { 1,5-bis(1,1-dimethylethyl)-3,3-dimethyl- } \\
\text { bicyclo[3.1.0]hexan-2-one }\end{array}$ & 11.82 & 0.50 \\
\hline n-propyl-benzamide & 12.01 & 8.0 \\
\hline $\begin{array}{l}\text { 2,6-bis(1,1-dimethylethyl)-2,5-cyclohexadiene-1,4- } \\
\text { dione }\end{array}$ & 12.30 & 0.80 \\
\hline 2-methyl-1,1'-biphenyl & 12.31 & 6.7 \\
\hline unknown & 12.39 & 7.7 \\
\hline unknown & 12.43 & 36 \\
\hline $\mathrm{N}, \mathrm{N}$-diethylbenzamide & 12.48 & 0.40 \\
\hline unknown & 12.51 & 1.9 \\
\hline 4-methyl-2,6-di(tert)butyl phenyol & 12.57 & 0.70 \\
\hline 1,2-dihydro-acenaphthylene & 12.60 & 1.5 \\
\hline
\end{tabular}


TABLE 10. SEMIQUANTITATIVE GC-MS RESULTS OF AIR SAMPLE FROM 1D1, TUBE 0953

\begin{tabular}{|c|c|c|}
\hline $\begin{array}{c}\text { Site 1D-1, Tube } 0953 \\
\text { Compound }\end{array}$ & $\begin{array}{c}\text { Retention Time } \\
\text { (minutes) }\end{array}$ & $\begin{array}{c}\text { Concentration } \\
\mathrm{pg} / \mathrm{L} \\
\end{array}$ \\
\hline C3 naphthalene & 12.75 & 3.2 \\
\hline diethyl phthalate & 12.84 & 52 \\
\hline diphenylamine & 12.92 & 0.30 \\
\hline diphenylmethanone & 12.97 & 1.5 \\
\hline 4-hydroxy-3,5-di-tert-butylbenzaldehyde & 13.66 & 0.20 \\
\hline isopropyl myristate & 13.81 & 0.10 \\
\hline trichlorobiphenyl & 13.91 & 0.30 \\
\hline phenanthrene & 14.32 & 83 \\
\hline trichlorobiphenyl & 14.57 & 0.80 \\
\hline tetrachlorobiphenyl & 14.77 & 0.60 \\
\hline tetrachlorobiphenyl (nc) & 14.84 & 0.20 \\
\hline trichlorobiphenyl & 14.87 & 1.4 \\
\hline hexadecanoic acid & 15.02 & 0.90 \\
\hline tetrachlorobiphenyl & 15.03 & 2.0 \\
\hline dibutyl phthalate & 15.10 & 61 \\
\hline dioctyl phthalate & 15.12 & 21 \\
\hline tetrachlorobiphenyl (nc) & 15.49 & 0.60 \\
\hline tetrachlorobiphenyl & 15.72 & 2.0 \\
\hline tetrachlorobiphenyl & 16.06 & 0.10 \\
\hline pentachlorobiphenyl & 16.34 & 0.0 \\
\hline pentachlorobiphenyl & 16.34 & 0.10 \\
\hline
\end{tabular}


TABLE 11. SEMIQUANTITATIVE GC-MS RESULTS OF AIR SAMPLE FROM E205, TUBE 0924

\begin{tabular}{|c|c|c|}
\hline $\begin{array}{c}\text { Site E205 S, Tube } 0924 \\
\text { Compound }\end{array}$ & $\begin{array}{c}\text { Retention Time } \\
\text { (minutes) }\end{array}$ & $\begin{array}{c}\text { Concentration } \\
\mathrm{pg} / \mathrm{L} \\
\end{array}$ \\
\hline trichlorofluoromethane & 2.35 & 100 \\
\hline hexane & 2.81 & 87 \\
\hline diisopropylamine & 3.24 & 0.20 \\
\hline trichloroethylene & 3.90 & 180 \\
\hline 4-methyl-2-pentanone & 4.45 & 1.3 \\
\hline toluene & 4.97 & 1400 \\
\hline 1-octene & 5.30 & 1.1 \\
\hline 3-heptanone & 5.39 & 45 \\
\hline tetrachloroethylene & 5.62 & 510 \\
\hline dimethyl benzene & 6.90 & 190 \\
\hline benzaldehyde & 7.82 & 390 \\
\hline propyl benzene & 7.84 & 39 \\
\hline dichlorobenzene & 8.31 & 1.1 \\
\hline 2,3,6-trimethyl octane & 8.50 & 3.4 \\
\hline C3 benzene & 8.68 & 15 \\
\hline acetophenone & 8.68 & 15 \\
\hline unknown & 8.89 & 3.9 \\
\hline triethyl ester phosphoric acid & 9.02 & 2.7 \\
\hline O,O-diethyl-S-ethylphosphorothioate & 9.42 & 0.0 \\
\hline unknown & 9.49 & 5.7 \\
\hline decahydro-2-methyl naphthalene & 9.61 & 2.9 \\
\hline unknown & 9.69 & 11 \\
\hline benzoic acid & 9.90 & 190 \\
\hline naphthalene & 10.08 & 19 \\
\hline
\end{tabular}


TABLE 11. SEMIQUANTITATIVE GC-MS RESULTS OF AIR SAMPLE FROM E205, TUBE 0924

\begin{tabular}{|c|c|c|}
\hline $\begin{array}{l}\text { Site E205 S, Tube } 0924 \\
\text { Compound } \\
\end{array}$ & $\begin{array}{c}\text { Retention Time } \\
\text { (minutes) }\end{array}$ & $\begin{array}{c}\text { Concentration } \\
\text { pg/L }\end{array}$ \\
\hline unknown & 10.42 & 2.7 \\
\hline unknown & 10.47 & 0.40 \\
\hline unknown & 10.54 & 6.4 \\
\hline benzothiazole & 10.63 & 7.8 \\
\hline 1-methyl naphthalene & 10.71 & 5.4 \\
\hline 2 -[bis[1-isopropyl]aminoethanol & 10.72 & 1.8 \\
\hline $\mathrm{N}, \mathrm{N}$-dibutylformamide & 10.72 & 1.8 \\
\hline 2-methyl naphthalene & 10.87 & 2.1 \\
\hline unknown & 11.28 & 2.8 \\
\hline 1,6-dimethylnaphthalene & 11.60 & 1.7 \\
\hline biphenyl & 11.60 & 140 \\
\hline trichlorophenol & 11.64 & 0.20 \\
\hline n-propyl-benzamide & 11.70 & 240 \\
\hline $1,1^{\prime}$-oxybis-benzene & 11.72 & 160 \\
\hline $\mathrm{N}, \mathrm{N}$-diethylbenzamide & 11.97 & 0.50 \\
\hline C3 naphthalene & 12.18 & 1.2 \\
\hline $\begin{array}{l}\text { 1,5-bis(1,1-dimethylethyl)-3,3-dimethyl- } \\
\text { bicyclo[3.1.0]hexan-2-one }\end{array}$ & 12.23 & 0.20 \\
\hline 1,2-dihydro-acenaphthylene & 12.23 & 0.50 \\
\hline 2-methyl-1,1'-biphenyl & 12.27 & 0.20 \\
\hline $\begin{array}{l}\text { 2,6-bis(1,1-dimethylethyl)-2,5-cyclohexadiene-1,4- } \\
\text { dione }\end{array}$ & 12.30 & 0.40 \\
\hline 4-methyl-2,6-di(tert)butyl phenyol & 12.57 & 0.0 \\
\hline unknown & 12.62 & 62 \\
\hline unknown & 12.84 & 22 \\
\hline
\end{tabular}


TABLE 11. SEMIQUANTITATIVE GC-MS RESULTS OF AIR SAMPLE FROM E205, TUBE 0924

\begin{tabular}{|c|c|c|}
\hline $\begin{array}{c}\text { Site E205 S, Tube } 0924 \\
\text { Compound } \\
\end{array}$ & $\begin{array}{c}\text { Retention Time } \\
\text { (minutes) }\end{array}$ & $\begin{array}{c}\text { Concentration } \\
\mathrm{pg} / \mathrm{L}\end{array}$ \\
\hline diethyl phthalate & 12.84 & 130 \\
\hline diphenylamine & 12.92 & 0.20 \\
\hline diphenylmethanone & 13.04 & 1.7 \\
\hline unknown & 13.07 & 0.30 \\
\hline isopropyl myristate & 13.75 & 2.8 \\
\hline trichlorobiphenyl & 13.91 & 0.50 \\
\hline 4-hydroxy-3,5-di-tert-butylbenzaldehyde & 14.10 & 0.10 \\
\hline phenanthrene & 14.32 & 42 \\
\hline dibutyl phthalate & 14.49 & 3.9 \\
\hline trichlorobiphenyl & 14.57 & 1.1 \\
\hline tetrachlorobiphenyl & 14.77 & 1.1 \\
\hline trichlorobiphenyl & 14.87 & 2.3 \\
\hline tetrachlorobiphenyl (nc) & 14.92 & 0.90 \\
\hline tetrachlorobiphenyl & 15.03 & 4.7 \\
\hline hexadecanoic acid & 15.08 & 1.0 \\
\hline dioctyl phthalate & 15.12 & 16 \\
\hline tetrachlorobiphenyl & 15.41 & 3.5 \\
\hline tetrachlorobiphenyl & 15.72 & 4.2 \\
\hline tetrachlorobiphenyl & 16.03 & 0.30 \\
\hline pentachlorobiphenyl & 16.29 & 0.50 \\
\hline pentachlorobiphenyl & 16.30 & 0.30 \\
\hline
\end{tabular}


TABLE 12. SEMIQUANTITATIVE GC-MS RESULTS OF AIR SAMPLE FROM 1I1, TUBE 0365

\begin{tabular}{|c|c|c|}
\hline $\begin{array}{c}\text { Site 1I-1, Tube } 0365 \\
\text { Compound } \\
\end{array}$ & $\begin{array}{c}\text { Retention Time } \\
\text { (minutes) }\end{array}$ & $\begin{array}{c}\text { Concentration } \\
\mathrm{pg} / \mathrm{L} \\
\end{array}$ \\
\hline trichlorofluoromethane & 2.35 & 61 \\
\hline hexane & 2.81 & 35 \\
\hline diisopropylamine & 3.35 & 11 \\
\hline trichloroethylene & 3.91 & 100 \\
\hline 4-methyl-2-pentanone & 4.26 & 0.40 \\
\hline toluene & 4.99 & 1100 \\
\hline 1-octene & 5.26 & 1.8 \\
\hline 3-heptanone & 5.39 & 160 \\
\hline tetrachloroethylene & 5.62 & 310 \\
\hline dimethyl benzene & 6.90 & 310 \\
\hline benzaldehyde & 7.81 & 330 \\
\hline propyl benzene & 7.84 & 110 \\
\hline dichlorobenzene & 8.42 & 11 \\
\hline C3 benzene & 8.47 & 100 \\
\hline 2,3,6-trimethyl octane & 8.48 & 3.2 \\
\hline acetophenone & 8.68 & 12 \\
\hline unknown & 8.89 & 8.2 \\
\hline triethyl ester phosphoric acid & 9.29 & 97 \\
\hline decahydro-2-methyl naphthalene & 9.66 & 0.90 \\
\hline O,O-diethyl-S-ethylphosphorothioate & 9.66 & 24 \\
\hline unknown & 9.90 & 7.5 \\
\hline benzoic acid & 9.92 & 680 \\
\hline unknown & 10.04 & 5.4 \\
\hline naphthalene & 10.07 & 24 \\
\hline
\end{tabular}


TABLE 12. SEMIQUANTITATIVE GC-MS RESULTS OF AIR SAMPLE FROM 1I1, TUBE 0365

\begin{tabular}{|c|c|c|}
\hline $\begin{array}{l}\text { Site 1I-1, Tube } 0365 \\
\text { Compound }\end{array}$ & $\begin{array}{l}\text { Retention Time } \\
\text { (minutes) }\end{array}$ & $\begin{array}{c}\text { Concentration } \\
\mathrm{pg} / \mathrm{L}\end{array}$ \\
\hline unknown & 10.07 & 16 \\
\hline benzothiazole & 10.47 & 67 \\
\hline unknown & 10.54 & 3.5 \\
\hline 2-methyl naphthalene & 10.88 & 7.6 \\
\hline $\mathrm{N}, \mathrm{N}$-dibutylformamide & 10.88 & 140 \\
\hline 2 -[bis[1-isopropyl]amino ethanol & 10.88 & 140 \\
\hline unknown & 10.91 & 1.5 \\
\hline trichlorophenol & 11.12 & 0.30 \\
\hline 1-methyl naphthalene & 11.14 & 42 \\
\hline unknown & 11.28 & 7.2 \\
\hline 1,6-dimethylnaphthalene & 11.60 & 1.3 \\
\hline 1,1'-oxybis-benzene & 11.72 & 14 \\
\hline biphenyl & 11.81 & 1.3 \\
\hline $\begin{array}{l}\text { 1,5-bis(1,1-dimethylethyl)-3,3-dimethyl- } \\
\text { bicyclo[3.1.0]hexan-2-one }\end{array}$ & 11.99 & 33 \\
\hline n-propyl-benzamide & 12.08 & 9.8 \\
\hline $\begin{array}{l}\text { 2,6-bis(1,1-dimethylethyl)-2,5-cyclohexadiene-1,4- } \\
\text { dione }\end{array}$ & 12.31 & 1.7 \\
\hline 2-methyl-1,1'-biphenyl & 12.31 & 5.6 \\
\hline N,N-diethylbenzamide & 12.47 & 0.40 \\
\hline unknown & 12.51 & 1.6 \\
\hline 1,2-dihydro-acenaphthylene & 12.57 & 4.5 \\
\hline 4-methyl-2,6-di(tert)butyl phenyol & 12.58 & 0.40 \\
\hline C3 naphthalene & 12.75 & 3.5 \\
\hline unknown & 12.79 & 2.3 \\
\hline
\end{tabular}


TABLE 12. SEMIQUANTITATIVE GC-MS RESULTS OF AIR SAMPLE FROM 1I1, TUBE 0365

\begin{tabular}{|c|c|c|}
\hline $\begin{array}{c}\text { Site } 11-1, \text { Tube } 0365 \\
\text { Compound }\end{array}$ & $\begin{array}{c}\text { Retention Time } \\
\text { (minutes) }\end{array}$ & $\begin{array}{c}\text { Concentration } \\
\mathrm{pg} / \mathrm{L}\end{array}$ \\
\hline unknown & 12.83 & 18 \\
\hline diethyl phthalate & 12.83 & 108 \\
\hline diphenylamine & 12.85 & 1.5 \\
\hline diphenylmethanone & 12.97 & 1.7 \\
\hline isopropyl myristate & 13.76 & 2.4 \\
\hline trichlorobiphenyl & 13.91 & 0.20 \\
\hline 4-hydroxy-3,5-di-tert-butylbenzaldehyde & 14.16 & 0.40 \\
\hline trichlorobiphenyl & 14.16 & 1.7 \\
\hline phenanthrene & 14.31 & 56 \\
\hline tetrachlorobiphenyl & 14.85 & 0.20 \\
\hline trichlorobiphenyl & 14.88 & 0.90 \\
\hline dibutyl phthalate & 14.91 & 82 \\
\hline tetrachlorobiphenyl (nc) & 14.92 & 0.50 \\
\hline hexadecanoic acid & 15.03 & 1.2 \\
\hline dioctyl phthalate & 15.10 & 17 \\
\hline tetrachlorobiphenyl & 15.38 & 0.80 \\
\hline tetrachlorobiphenyl & 15.40 & 0.70 \\
\hline tetrachlorobiphenyl & 15.71 & 1.8 \\
\hline tetrachlorobiphenyl & 15.71 & 1.7 \\
\hline pentachlorobiphenyl & 16.22 & 0.10 \\
\hline pentachlorobiphenyl & 16.31 & 0.30 \\
\hline
\end{tabular}


TABLE 13. SEMIQUANTITATIVE GC-MS RESULTS OF AIR SAMPLE FROM 113, TUBE 0670

\begin{tabular}{|c|c|c|}
\hline $\begin{array}{c}\text { Site } 11-3, \text { Tube } 0670 \\
\text { Compound }\end{array}$ & $\begin{array}{c}\text { Retention Time } \\
\text { (minutes) }\end{array}$ & $\begin{array}{c}\text { Concentration } \\
\mathrm{pg} / \mathrm{L} \\
\end{array}$ \\
\hline trichlorofluoromethane & 2.41 & 3.7 \\
\hline hexane & 2.88 & 2.9 \\
\hline diisopropylamine & 3.37 & 0.90 \\
\hline trichloroethylene & 3.90 & 31 \\
\hline 4-methyl-2-pentanone & 4.44 & 16 \\
\hline toluene & 4.97 & 1000 \\
\hline 1-octene & 5.29 & 0.70 \\
\hline 3-heptanone & 5.39 & 70 \\
\hline tetrachloroethylene & 5.61 & 120 \\
\hline dimethyl benzene & 6.90 & 410 \\
\hline benzaldehyde & 7.81 & 280 \\
\hline propyl benzene & 7.84 & 120 \\
\hline dichlorobenzene & 8.42 & 11 \\
\hline C3 benzene & 8.47 & 100 \\
\hline acetophenone & 8.68 & 11 \\
\hline unknown & 8.89 & 9.3 \\
\hline triethyl ester phosphoric acid & 9.31 & 100 \\
\hline unknown & 9.52 & 6.1 \\
\hline decahydro-2-methyl naphthalene & 9.61 & 6.7 \\
\hline O,O-diethyl-S-ethylphosphorothioate & 9.67 & 59 \\
\hline unknown & 9.67 & 10 \\
\hline benzoic acid & 9.92 & 610 \\
\hline unknown & 10.09 & 7.0 \\
\hline naphthalene & 10.12 & 190 \\
\hline
\end{tabular}


TABLE 13. SEMIQUANTITATIVE GC-MS RESULTS OF AIR SAMPLE FROM 1I3, TUBE 0670

\begin{tabular}{|c|c|c|}
\hline $\begin{array}{l}\text { Site } 11-3, \text { Tube } 0670 \\
\text { Compound }\end{array}$ & $\begin{array}{l}\text { Retention Time } \\
\text { (minutes) }\end{array}$ & $\begin{array}{c}\text { Concentration } \\
\mathrm{pg} / \mathrm{L}\end{array}$ \\
\hline unknown & 10.17 & 140 \\
\hline benzothiazole & 10.25 & 6.1 \\
\hline unknown & 10.47 & 1.3 \\
\hline $\mathrm{N}, \mathrm{N}$-dibutylformarmide & 10.88 & 140 \\
\hline 2-[bis[1-isopropyl] aminoethanol & 10.88 & 140 \\
\hline 2-methyl naphthalene & 10.88 & 5.7 \\
\hline 1-methyl naphthalene & 11.14 & 36 \\
\hline unknown & 11.29 & 6.9 \\
\hline trichlorophenol & 11.63 & 0.60 \\
\hline 1,1'-oxybis-benzene & 11.72 & 14 \\
\hline $\begin{array}{l}\text { 1,5-bis(1,1-dimethylethyl)-3,3-dimethyl- } \\
\text { bicyclo[3.1.0]hexan-2-one }\end{array}$ & 11.78 & 0.50 \\
\hline biphenyl & 11.81 & 1.2 \\
\hline $\mathrm{N}, \mathrm{N}$-diethylbenzamide & 11.92 & 0.10 \\
\hline n-propyl-benzamide & 12.08 & 4.7 \\
\hline 1,6-dimethylnaphthalene & 12.17 & 3.3 \\
\hline 1,2-dihydro-acenaphthylene & 12.20 & 1.5 \\
\hline 2-methyl-1,1'-biphenyl & 12.31 & 5.7 \\
\hline $\begin{array}{l}\text { 2,6-bis(1,1-dimethylethyl)-2,5-cyclohexadiene- } \\
\text { 1,4-dione }\end{array}$ & 12.31 & 1.5 \\
\hline unknown & 12.43 & 9.1 \\
\hline 4-methyl-2,6-di(tert)butyl phenyol & 12.58 & 0.50 \\
\hline C3 naphthalene & 12.75 & 3.4 \\
\hline unknown & 12.84 & 8.6 \\
\hline diethyl phthalate & 12.84 & 74 \\
\hline
\end{tabular}


TABLE 13. SEMIQUANTITATIVE GC-MS RESULTS OF AIR SAMPLE FROM 1I3, TUBE 0670

\begin{tabular}{|c|c|c|}
\hline $\begin{array}{c}\text { Site 1I-3, Tube } 0670 \\
\text { Compound }\end{array}$ & $\begin{array}{c}\text { Retention Time } \\
\text { (minutes) }\end{array}$ & $\begin{array}{c}\text { Concentration } \\
\mathrm{pg} / \mathrm{L} \\
\end{array}$ \\
\hline diphenylmethanone & 12.97 & 1.5 \\
\hline unknown & 13.05 & 0.40 \\
\hline diphenylamine & 13.40 & 2.4 \\
\hline 4-hydroxy-3,5-di-tert-butylbenzaldehyde & 13.63 & 0.20 \\
\hline phenanthrene & 14.33 & 60 \\
\hline isopropyl myristate & 14.43 & 0.30 \\
\hline trichlorobiphenyl & 14.47 & 0.30 \\
\hline trichlorobiphenyl & 14.58 & 0.60 \\
\hline tetrachlorobiphenyl & 14.77 & 0.30 \\
\hline trichlorobiphenyl & 14.88 & 0.90 \\
\hline dibutyl phthalate & 14.92 & 30 \\
\hline tetrachlorobiphenyl (nc) & 14.92 & 0.30 \\
\hline tetrachlorobiphenyl & 15.09 & 0.70 \\
\hline tetrachlorobiphenyl & 15.09 & 0.70 \\
\hline dioctyl phthalate & 15.11 & 17 \\
\hline hexadecanoic acid & 15.16 & 0.80 \\
\hline tetrachlorobiphenyl & 15.41 & 1.6 \\
\hline tetrachlorobiphenyl & 15.41 & 0.80 \\
\hline pentachlorobiphenyl & 15.58 & 0.0 \\
\hline pentachlorobiphenyl & 16.62 & 0.10 \\
\hline
\end{tabular}


TABLE 14. SEMIQUANTITATIVE GC-MS RESULTS OF AIR SAMPLE FROM 132, TUBE 0929

\begin{tabular}{|c|c|c|}
\hline $\begin{array}{c}\text { Site } 132, \text { Tube } 0929 \\
\text { Compound }\end{array}$ & $\begin{array}{l}\text { Retention Time } \\
\text { (minutes) }\end{array}$ & $\begin{array}{c}\text { Concentration } \\
\operatorname{pg} / \mathbf{L}\end{array}$ \\
\hline trichlorofluoromethane & 2.36 & 74 \\
\hline hexane & 2.81 & 27 \\
\hline chloroform & 3.06 & 300 \\
\hline diisopropylamine & 3.31 & 600 \\
\hline trichloroethylene & 3.90 & 57 \\
\hline 4-methyl-2-pentanone & 4.40 & 4.3 \\
\hline toluene & 4.95 & 1700 \\
\hline 1-octene & 5.19 & 1.9 \\
\hline 3-heptanone & 5.42 & 0.0 \\
\hline tetrachloroethylene & 5.63 & 560 \\
\hline benzaldehyde & 6.45 & 210 \\
\hline dimethyl benzene & 6.56 & 57 \\
\hline 1,4-oxathiane & 6.60 & 46 \\
\hline propyl benzene & 7.68 & 30 \\
\hline acetophenone & 8.15 & 280 \\
\hline trimethyl octane & 8.30 & 1.1 \\
\hline limonene & 8.32 & 14 \\
\hline dichlorobenzene & 8.43 & 12 \\
\hline methyl ethyl benzene & 8.47 & 100 \\
\hline
\end{tabular}


TABLE 14. SEMIQUANTITATIVE GC-MS RESULTS OF AIR SAMPLE FROM 132, TUBE 0929

\begin{tabular}{|c|c|c|}
\hline $\begin{array}{c}\text { Site } 132, \text { Tube } 0929 \\
\text { Compound }\end{array}$ & $\begin{array}{l}\text { Retention Time } \\
\text { (minutes) }\end{array}$ & $\begin{array}{c}\text { Concentration } \\
\text { pg/L }\end{array}$ \\
\hline unknown & 8.48 & 7.8 \\
\hline triethyl ester phosphoric acid & 8.60 & 0.30 \\
\hline decahydro-2-methyl naphthalene & 9.18 & 1.3 \\
\hline unknown & 9.20 & 29 \\
\hline unknown & 9.42 & 12 \\
\hline unknown & 9.52 & 2.4 \\
\hline benzoic acid & 9.53 & 5.3 \\
\hline O,O-diethyl-S-ethylphosphorothioate & 9.68 & 18 \\
\hline unknown & 10.00 & 2.6 \\
\hline trichlorobenzene & 10.01 & 37 \\
\hline unknown & 10.08 & 4.9 \\
\hline 1-methyl naphthalene & 10.26 & 9.0 \\
\hline benzothiazole & 10.48 & 73 \\
\hline $\mathrm{N}, \mathrm{N}$-dibutylformamide & 10.62 & 2.7 \\
\hline 2 -[bis[1-isopropyl]amino ethanol & 10.63 & 2.7 \\
\hline biphenyl & 10.79 & 1.0 \\
\hline trichlorophenol & 11.03 & 0.10 \\
\hline $\mathrm{N}$-propyl benzamide & 11.05 & 2.0 \\
\hline 2-methyl naphthalene & 11.16 & 45 \\
\hline
\end{tabular}


TABLE 14. SEMIQUANTITATIVE GC-MS RESULTS OF AIR SAMPLE FROM 132, TUBE 0929

\begin{tabular}{|c|c|c|}
\hline $\begin{array}{l}\text { Site 132, Tube } 0929 \\
\text { Compound }\end{array}$ & $\begin{array}{l}\text { Retention Time } \\
\text { (minutes) }\end{array}$ & $\begin{array}{c}\text { Concentration } \\
\operatorname{pg} / \mathrm{L}\end{array}$ \\
\hline $\begin{array}{l}\text { 1,5-bis(1,1-dimethylethyl)-3,3-dimethyl- } \\
\text { bicyclo[3.1.0]hexan-2-one }\end{array}$ & 11.26 & 0.50 \\
\hline $1,1^{\prime}$-oxybisbenzene & 11.41 & 0.10 \\
\hline 1,6-dimethylnaphthalene & 11.53 & 0.50 \\
\hline C3 naphthalene & 11.61 & 3.7 \\
\hline $\begin{array}{l}\text { 2,6-bis(1,1-dimethylethyl)-2,5- } \\
\text { cyclohexadiene-1,4-dione }\end{array}$ & 11.63 & 0.10 \\
\hline 2-methyl-1,1'-biphenyl & 11.70 & 1.4 \\
\hline $\mathrm{N}, \mathrm{N}$-diethylbenzamide & 11.84 & 0.30 \\
\hline unknown & 11.88 & 0.40 \\
\hline 1,2-dihydro-acenaphthylene & 11.96 & 1.7 \\
\hline 4-methyl-2,6-di(tert)butyl phenol & 12.02 & 0.60 \\
\hline unknown & 12.22 & 36 \\
\hline diphenylmethanone & 12.33 & 0.70 \\
\hline diphenylamine & 12.81 & 1.5 \\
\hline diethyl phthalate & 12.84 & 140 \\
\hline trichlorobiphenyl & 13.31 & 0.10 \\
\hline 4-hydroxy-3,5-di-tert-butylbenzaldehyde & 13.40 & 0.10 \\
\hline isopropyl myristate & 13.65 & 0.20 \\
\hline trichlorobiphenyl & 13.82 & 0.0 \\
\hline
\end{tabular}


TABLE 14. SEMIQUANTITATIVE GC-MS RESULTS OF AIR SAMPLE FROM 132, TUBE 0929

\begin{tabular}{|l|c|c|}
\hline \multicolumn{1}{|c|}{$\begin{array}{c}\text { Site 132, Tube 0929 } \\
\text { Compound }\end{array}$} & $\begin{array}{c}\text { Retention Time } \\
\text { (minutes) }\end{array}$ & $\begin{array}{c}\text { Concentration } \\
\text { pg/L }\end{array}$ \\
\hline tetrachlorobiphenyl (nc) & 14.16 & 0.10 \\
\hline tetrachlorobiphenyl & 14.17 & 1.9 \\
\hline phenanthrene & 14.34 & 60 \\
\hline hexadecanoic acid & 14.45 & 0.30 \\
\hline dialkyl phthalate (butyl) & 14.49 & 3.6 \\
\hline tetrachlorobiphenyl & 14.55 & 0.0 \\
\hline dioctyl phthalate & 14.58 & 0.0 \\
\hline tetrachlorobiphenyl (nc) & 14.71 & 0.10 \\
\hline tetrachlorobiphenyl & 14.93 & 0.30 \\
\hline tetrachlorobiphenyl & 14.98 & 0.0 \\
\hline pentachlorobiphenyl & 15.31 & 0.30 \\
\hline diisooctylphthalate & 15.36 & 6.4 \\
\hline pentachlorobiphenyl & 15.84 & 0.10 \\
\hline
\end{tabular}


ENCLOSURE 1:

MASTER LIST OF POTENTIAL CONTAMINANTS OF PILOT PLANT

BUILDING E5625 
Enclosure 1

\title{
MASTER LIST OF POTENTIAL CONTAMINANTS OF PILOT PLANT BUILDING E5625
}

\section{Chemicals Possibly throughout the Bullding, trom 1941 to 1985}

\author{
1. PCBs \\ 2. lead (in paint) \\ 3. asbestos \\ 3-A. mercury
}

3-B. chromium salts

\section{Chemicals Possibly throughout the Bullding, from 1942 CC2 Production}
4. $\quad N, N^{\prime}, 2,2^{\prime}, 4,4^{\prime}, 6,6^{\prime}$-octachlorocarbanilide (CC2)
5. N,N-diphenylurea (DPU)
6. bis(trichlorophenylurea) (TCPU)
7. acetic acid/sodium acetate
8. chlorine
9. $\mathbf{N}, \mathbf{N}$-dichloro-bis(dichlorophenyl)urea
10. tetrachloroquinone
11. 1,1,2,2-tetrachloroethane
12. ethyl acetate
13. lead (cf \#2)
14. copper
15. zinc

Chemicals from Large-Scale GB Plloting, 1951-1956 or Longer, and Bench-Scale G-Agent Synthesis, Laboratories 2D2, 2F, 3D2, 4D1, 4D2, and $4 \mathrm{~F}$

8. chlorine

16. isopropyl methylphosphonofluoridate (GB)

17. methanol/methoxide

18. phosphorus trichloride (TH)

19. dimethyl hydrogen phosphite (DMHP)

20. hydrogen fluoride (or sometimes șodium fluoride?)

21. hydrogen chloride (hydrochloric acid)

22. chloromethane

23. dimethyl ether

24. monomethyl dihydrogen phosphite

25. phosphorous acid

26. "phosphates"

27. tributylamine

28. $\quad \mathrm{N}, \mathrm{N}^{\prime}$-diisopropylcarbodiimide 
28-A. Ni.N'-diisopropylurea

28-B. N,N'-dicyclohexylcarbodiimide

28-C. N.N'-dicyclohexylurea

29. xylene

30. carbon tetrachloride

31. trichloroethylene

32. Dowtherm A (73.5:26.5 diphenyl oxide/biphenyl)

33. monomethyl methylphosphonic acid ("phosphonic acid")

34. methylphosphonic acid anhydride ("pyrophosphonic acid")

35. methylphosphonyl dichloride ("dichloro")

36. methylphosphonyl difluoride (DF or "difluoro")

37. phosphorus oxychloride

38. isopropyl alcohol

39. sodium hydroxide (NW) (aqueous)

40. ethylene glycol

41. charcoal (exhausted adsorbent)

42. diisopropyl methylphosphonate (DIMP)

43. phosgene (CG)

44. isopropyl methylphosphonochloridate ("chloro-GB")

45. diisopropyl dimethylpyrophosphonate ("pyro")

46. dihydrogen dimethylpyrophosphonate ("pyro acid")

47. 2-chloropropane

48. isopropyl methylphosphonic acid ("i-acid") (or salts)

49. methylphosphonofluoridic acid

50. methylene chloride (?)

51. ethyl acetoacetate

52. pinacolyl methylphosphonofluoridate (GD)

53. cyclohexyl methylphosphonofluoridate (GF)

54. 2-methylcyclohexyl methylphosphonofluoridate (EA 1356)

55. trans-2-methylcyclohexyl methylphosphonotluoridate (EA 3534)

56. pinacolyl alcohol $\left[\mathrm{CH}\left(\mathrm{CH}_{3}\right)\left(\mathrm{C}\left[\mathrm{CH}_{3}\right] 3\right) \mathrm{OH}\right]$

57. cyclohexanol

58. cis/trans-2-methylcyclohexanol

59. trans-2-methylcyclohexanol

60. pinacolyl methylphosphonic acid (or salts)

61. cyclohexyl methylphosphonic acid (or salts)

62. cis/trans-2-methylcyclohexyl methylphosphonic acid (or salts)

63. trans-2-methylcyclohexyl methylphosphonic acid (or salts)

63-A. methylphosphonic acid (and salts)

Chemicals from Limited Production of "Difluoro", 1975-1985, Laboratories $3 E, 4 E$, and $4 F$

20. hydrogen fluoride (or sometimes sodium fluoride?)

35. methylphosphonyl dichloride ("dichloro")

36. methylphosphonyl difluoride (DF or "difluoro")

39. sodium hydroxide (NW) (aqueous) 
Chemicals from VX Production, 1975-1985, Roof over 3rd-Floor Hall and Maintenance Shop (Step 1), Rooms 2A and 3A (Step 2), Room 113 (Step 3 , Actual Conversion to $\mathrm{VX}$ )

21. hydrogen chloride (hydrochloric acid)

64. O-ethyl S-(2-diisopropylaminoethyl)

65. methylphosphonothiolate (VX)

66. ethanol (ZS)

67. butane and/or isobutane (RM)

68. Gulf BT (apparently a hydrocarbon mixture)

69. cumene (isopropylbenzene)

70. diethyl methylphosphonite (DEMP)

71. 2-diisopropylaminoethanol (KB)

72. ammonia (ZK)/ammonium chloride $(\mathrm{RX})$

73. sulfur

74. ethyl 2-diisopropylaminoethyl methylphosphonite (QL)

75. O-thyl 0-2-diisopropylaminoethyl methylphosphonothionate

76. ethyl hydrogen methylphosphonite

77. diaminomethylphosphine

78. chloroethane (JO)

79. bis(2-diisopropylaminoethyl) methylphosphonite (LT)

80. ethyl 2-diisopropylaminoethyl methylphosphonate

81. bis(2-diisopropylaminoethyl) methylphosphonate

82. ethyl methylphosphonic acid

83. methylphosphondiamide

84. Socony SV Light Oil

85. exhausted charcoal and particulate filters

85-A. 2-diisopropylamincethanethiol

85-B. O-ethyl methylphosphonothioate

85-C. bis(diisopropylaminoethyl) sulfide

85-D. O-ethyl methylphosphonic acid anhydride

85-E. diethyl methylphosphonate

Chemicals from Production of Binary Agent (GB and VX) Reaction Fluids, 1968-1985, Pipe Storage Room (plus Laboratories 2B, 3E,4B, 4E and 4F for Polysulfide)

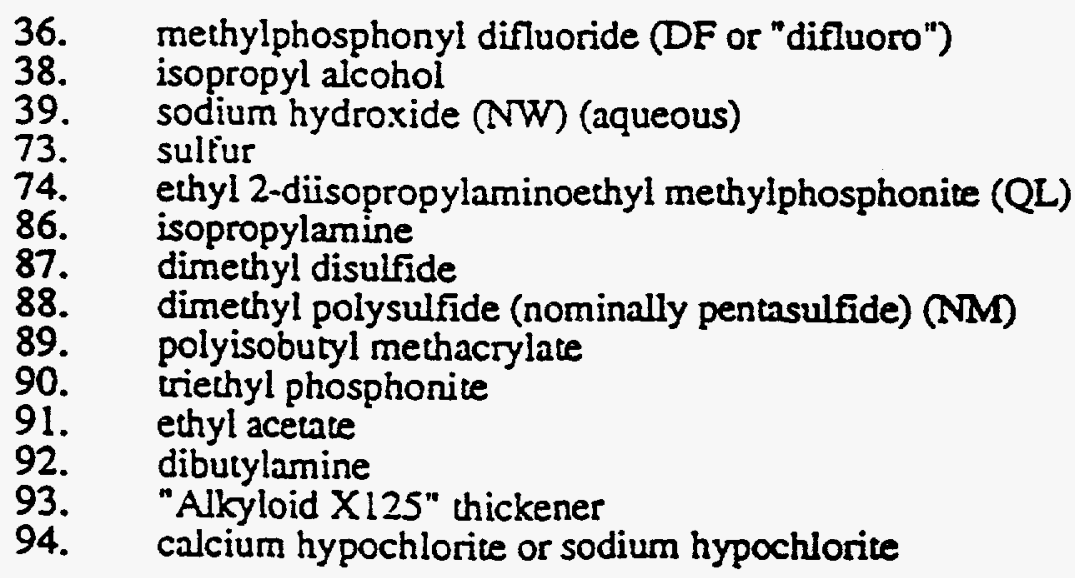


95. monoethanolamine

96. acetone

97. benzene

98. methanesultonate ion.

\section{Chemicals from Limited Production of Glycolate Agents, 1961-1971, Laboratories 2C, 3C, 4A}

17. methanol/methoxide

38. isopropyl alcohol

39. sodium hydroxide (NW) (aqueous)

40. ethylene glycol

99. 3-Quinuclidinyl Benzilate (BZ)

100. N-Methyl-4-Piperidyl Cyclopentylphenylglycolate (EA3443)

101. N-Methyl-4-Piperidyl Cyclobutylphenylglycolate (EA3580)

102. N-Methyl-4-Piperidyl Isopropylphenylglycolate (EA3834)

103. heptane

104. 3-quinuclidinol

105. sodium benzilate

106. N-methyl-4-piperidinol

107. Sodium cyclopentylphenylglycolate

108. Sodium cyclobutylphenylglycolate

109. sodium isopropylphenylglycolate

109-A. methyl benzilate

109-B. methyl cyclopentylphenylglycolate

109-C. methyl cyclobutylphenylglycolate

109-D. methyl isopropylphenylglycolate

Chemicals Involved In B-1 Dye Production, 1978-1984, Laboratories 2B and $3 B$

7. acetic acid/sodium acetate

21. hydrogen chloride (hydrochloric acid)

66. ethanol (ZS)

110. 1-p-nitrophenyl-2-aminonaphthalene (B-1 Dye)

111. p-nitrobenzenediazonium salts

112. 2-amino-1-naphthalenesulfonic acid (Tobias acid)

113. p-nitroaniline

114. sulfamic acid

115. Sodium nitrite

116. 2-ethoxyethanol

Chemicals Involved in BBC Production, 1966, Laboratory 4B

39. sodium hydroxide (NW) (aqueous)

66. ethanol (ZS)

117. alpha-bromobenzyl cyanide

118. benzyl chloride

119. benzyl cyanide

120. bromine 
121. hydrogen bromide

122. sodium bromate

123. sodium cyanide (or pocassium cyanide)

124. sulfuric acid

\section{XR (Botulinum Toxin from Clostridlum botullnum) (Place and Time Not Known)}

126. botulinum toxin (XR) in fermentation broth

Lysergic Acid and LSD (Lysergic Acld Diethylamide), 1964-1966, Laboratory $4 \mathrm{C}$

127. lysergic acid

128. lysergic acid diethylamide agent thickeners

129. polyisobutyl methacrylate

130. Alkyloid $X 125$

Chemicals Involved in Filling Operations, 1948-1985, 11 Laboratory Complex (111, 112, 113, 114, and 115)

16. isopropyl methylphosphonofluoridate (GB)

38. isopropyl alcohol

39. sodium hydroxide (NW) (aqueous)

40. ethylene glycol

52. pinacolyl methylphosphonofluoridate (GD)

53. cyclohexyl methylphosphonofluoridate (GF)

54. 2-methylcyclohexyl methylphosphonofluoridate (EA 1356)

55. trans-2-methylcyclohexyl methylphosphonofluoridate (EA 3534)

64. O-ethyl S-(2-diisopropylaminoethyl) methylphosphonothiolate (VX)

94. calcium hypochlorite or sodium hypochlorite

123. sodium cyanide (or potassium cyanide)

131. O-ethyl N,N-dimethylphosphoramidocyanidate (GA)

131-A. O-ethyl N,N-dimethylphosphoramidic acid

131-B. ethyl phosphorocyanidic acid

132. bis(2-chloroethyl) sulfide ( $H$ or $H D)$

132-A. bis(2-chloroethyl) sulfoxide

132-B bis(2-chloroethyl) sulfone

132-C 2-hydroxyethyl 2'-chloroethyl sulfide

132-D. 2-hydroxyethyl 2'-chloroethyl sulfoxide

132-E. 2-hydroxyethyl 2'-chloroethyl sulfone

132-F. thiodiglycol

132-G. bis(2-hydroxyethyl) sulfoxide

132-H. bis(2-hydroxyethyl) sulfone

132-I. 2-chloroethyl vinyl sulfide

132-J. 2-chloroethyl vinyl sulfoxide

132-J. 2-chloroethyl vinyl sulfone

$132-K$. divinyl sulfide

132-L. divinyl sulfoxide 
132-M. divinyl sulfone

132-N. 1.4-dithiane

132-0. 1.4-thioxane

Chemicals Involved in Agent Transfer Operations, 1970-1985, 11

Laboratory Complex (111, 112, 113, 114, and 115)

16. isopropyl methylphosphonofluoridate (GB)

39. sodium hydroxide (NW) (aqueous)

43. phosgene (CG)

52. pinacolyl mechylphosphonofluoridate (GD)

54. 2-methylcyclohexyl methylphosphonofluoridate (EA 1356)

64. O-thyl S-(2-diisopropylaminoethyl) methylphosphonothiolate $(\mathrm{VX})$

94. calcium hypochlorite or sodium hypochlorite

131. O-ethyl N,N-dimethylphosphoramidocyanidate (GA)

132. bis(2-chloroethyl) sulfide (H or $\mathrm{HD}$ )

133. mixture of bis(2-chloroethylthioethyl) ether (T) with mustard (HD) (\#132) - known as HT

134. 2-chlorovinyldichlorarsine (lewisite or $\mathrm{L}$ )

134-A. 2-chlorovinylarsonic acid

134-B. inorganic arsenic salts or oxide

135. cyanogen chloride (CK)

Chemicals Involved in Bulk Detoxiflcation, 1970-1985, Laboratorles 111, 114 , and 115

39. sodium hydroxide (NW) (aqueous)

43. phosgene (CG)

94. calcium hypochlorite or sodium hypochlorite

132. bis(2-chloroethy1) sulfide (H or HD)

134. 2-chlorovinyldichlorarsine (lewisite or $\mathrm{L}$ )

135. cyanogen chloride (CK)

136. nitrogen mustards (HN) (HN-1 and $\mathrm{HN}-3$ )

137. trichloronitromethane (chloropicrin or PS)

138. hydrogen cyanide (AC) (see \#123)

Antiriot Agents CS, CS-1 and CS-2, Filling Operations, 1973-1975, Laboratories $1 \mathrm{G}$ and $1 \mathrm{H}$

39. sodium hydroxide (NW) (aqueous)

139. O-chlorobenzylidenemalononitrile (CS), pure or coated with agents to prevent sticking

139-A. O-chlorobenzaldehyde

139-B. malononitrile

Filling Operations, 1973-1975, 11 Laboratory Complex (111, 112, 113, 114, and 115)

39. sodium hydroxide (NW) (aqueous) 
43. phosgene (CG)

135. cyanogen chloride (CK)

137. trichloronitromethane (chloropicrin or PS)

Pilot Plant Incineration of War Gas Identification Sets, 1976-1977, Laboratories $1 \mathrm{C}$ and 115

16. isopropyl methylphosphonofluoridate (GB)

39. sodium hydroxide (NW) (aqueous)

43. phosgene (CG)

123. Sodium cyanide (or potassium cyanide)

132. bis(2-chloroethyl) sulfide ( $\mathrm{H}$ or $\mathrm{HD})$

134. 2-chlorovinyldichlorarsine (lewisite or L)

135. cyanogen chloride (CK)

136. nitrogen mustards (HN) (HN-1 and HN-3)

137. trichloronitromethane (chloropicrin or PS)

140. chloroacetophenone (CN)

141. phenarsazine chloride (adamsite or DM)

142. triphosgene (CG simulant)

143. hexamethylene glycol (G-simulant)

144. 2-methoxyethanol (G-simulant)

145. tetrahydrofurfuryl alcohol

146. N-methylglucamine ( $V$-simulant)

147. diethylene glycol $(V$-simulant)

148. isoamyl salycilate (H-simulant)

CR Dilution and Filling, 1979-1980, Laboratory 1A

149. dibenzo[b,f]-1,4-oxazepine (CR)

150. propylene glycol

Incineration of CR Solutions, 1981, Laboratory 1C

149. dibenzo[b,f]-1,4-oxazepine (CR)

150. propylene glycol

Incineration of CK, 1981, Laboratories $1 \mathrm{C}$ and 115

39. sodium hydroxide (NW) (aqueous)

135. cyanogen chloride (CK)

Toxic Base-Catalyzed Decomposition Products of VX and $L, 1975-1985$, 11 Laboratory Complex (111, 112, 113, 114, and 115) and Laboratory $1 C$

151. bis(diisopropylaminoethyl) disulfide (EA4196)

152. S-diisopropylaminoethyl methylphosponothioic acid (EA2192)

153. 2-chlorovinylarsenoxide (ewisite oxide) 
Appendix C:

Floor Plans for Buildings

Monitored at the Pilot Plant Complex 


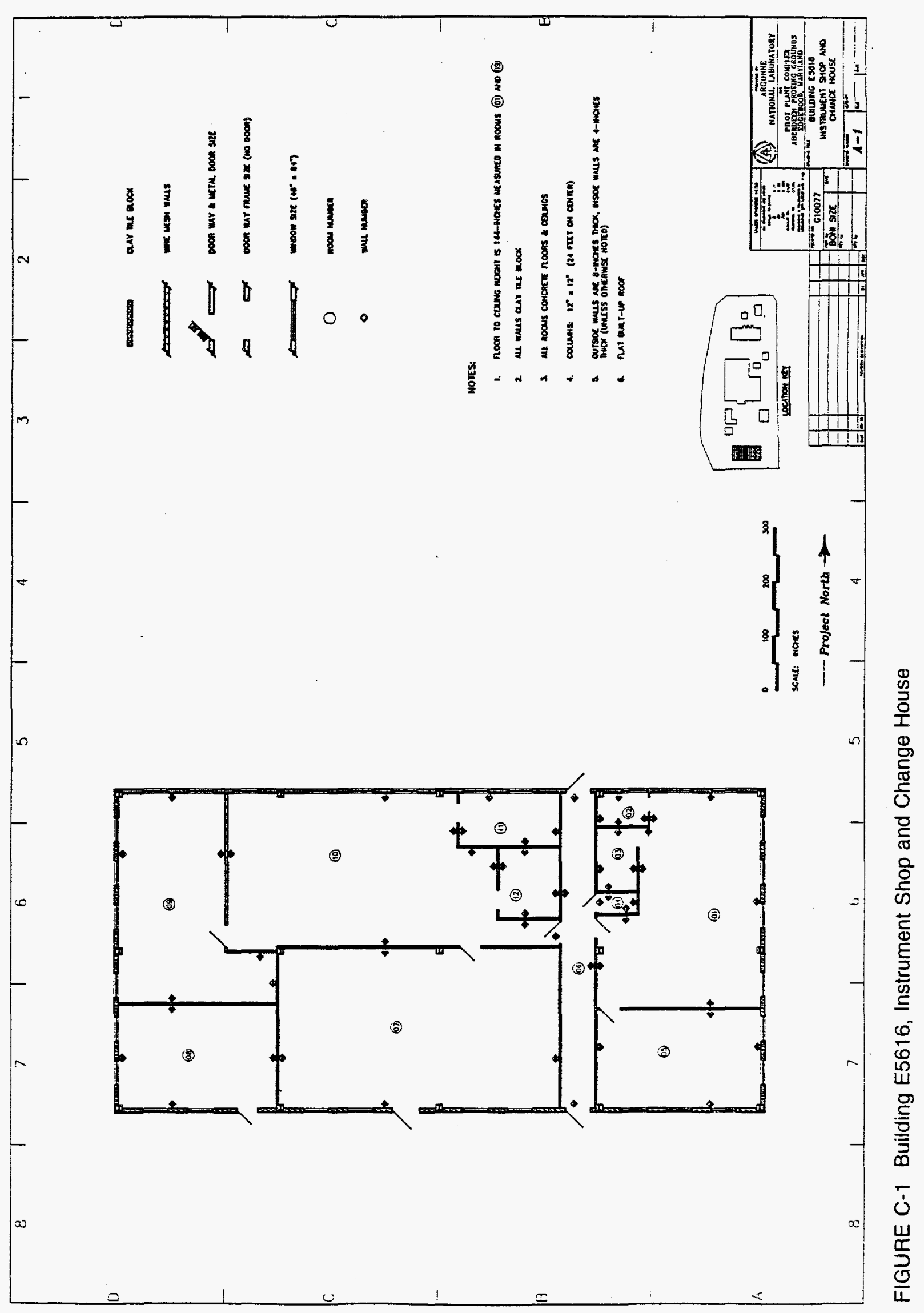




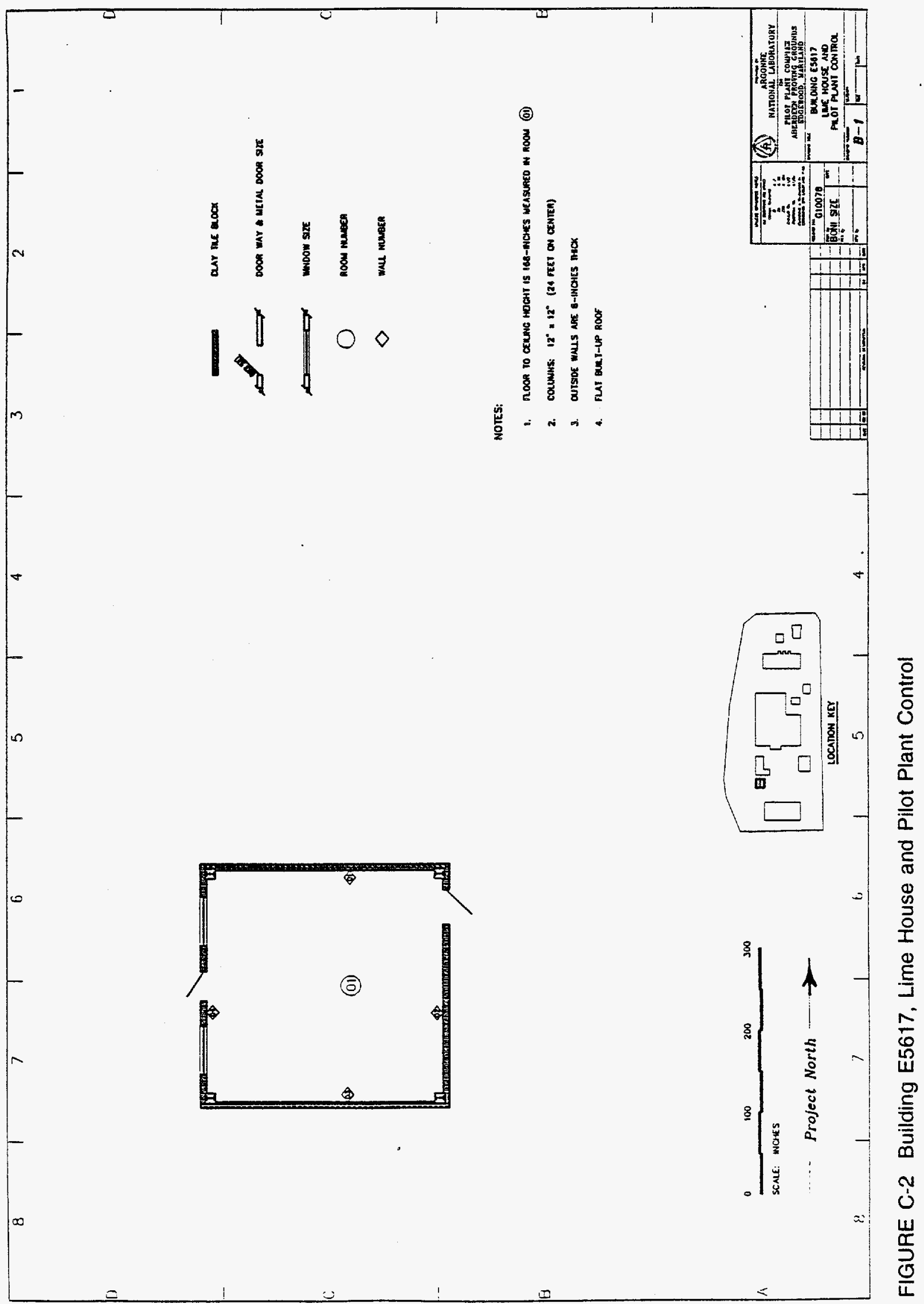




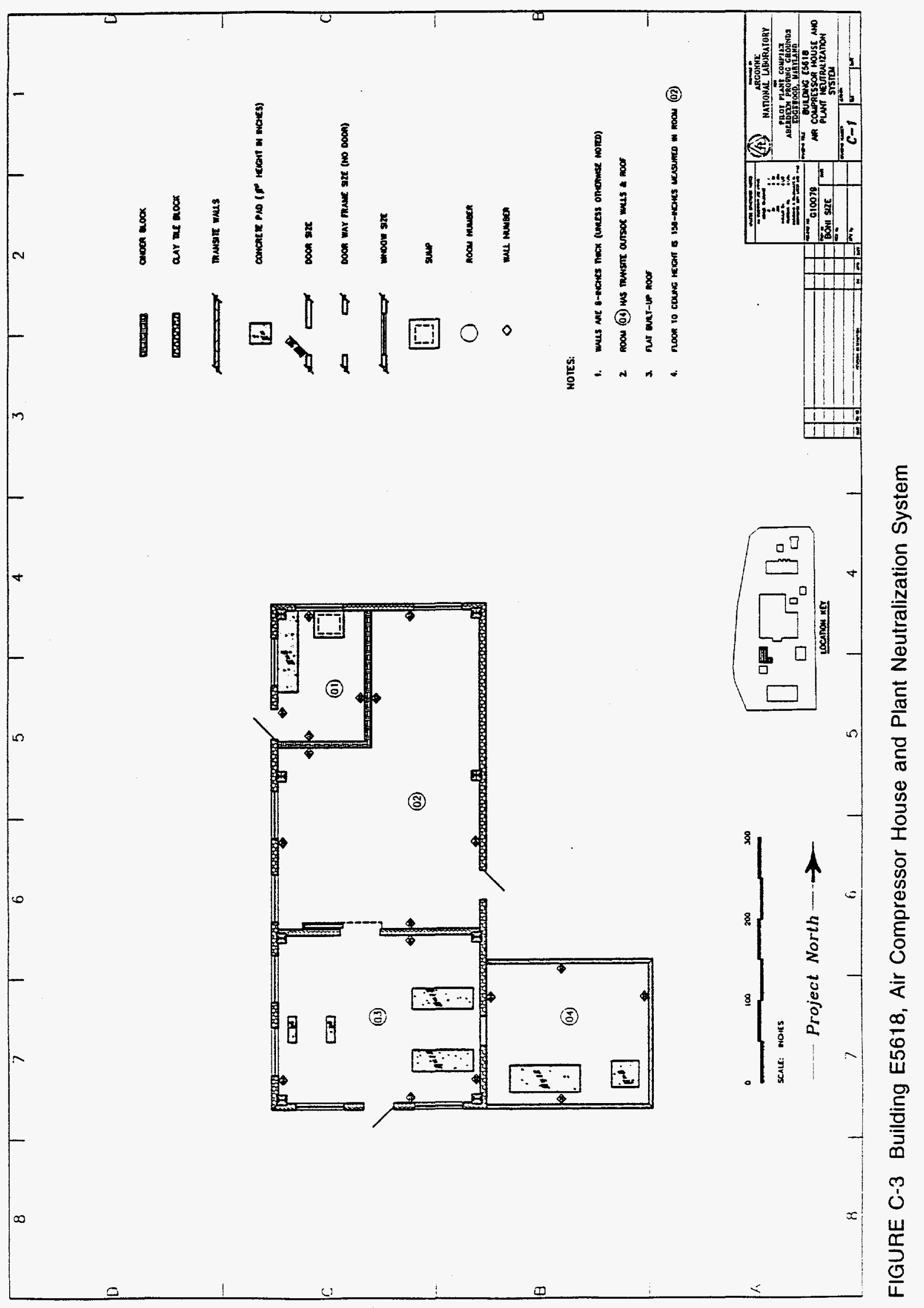




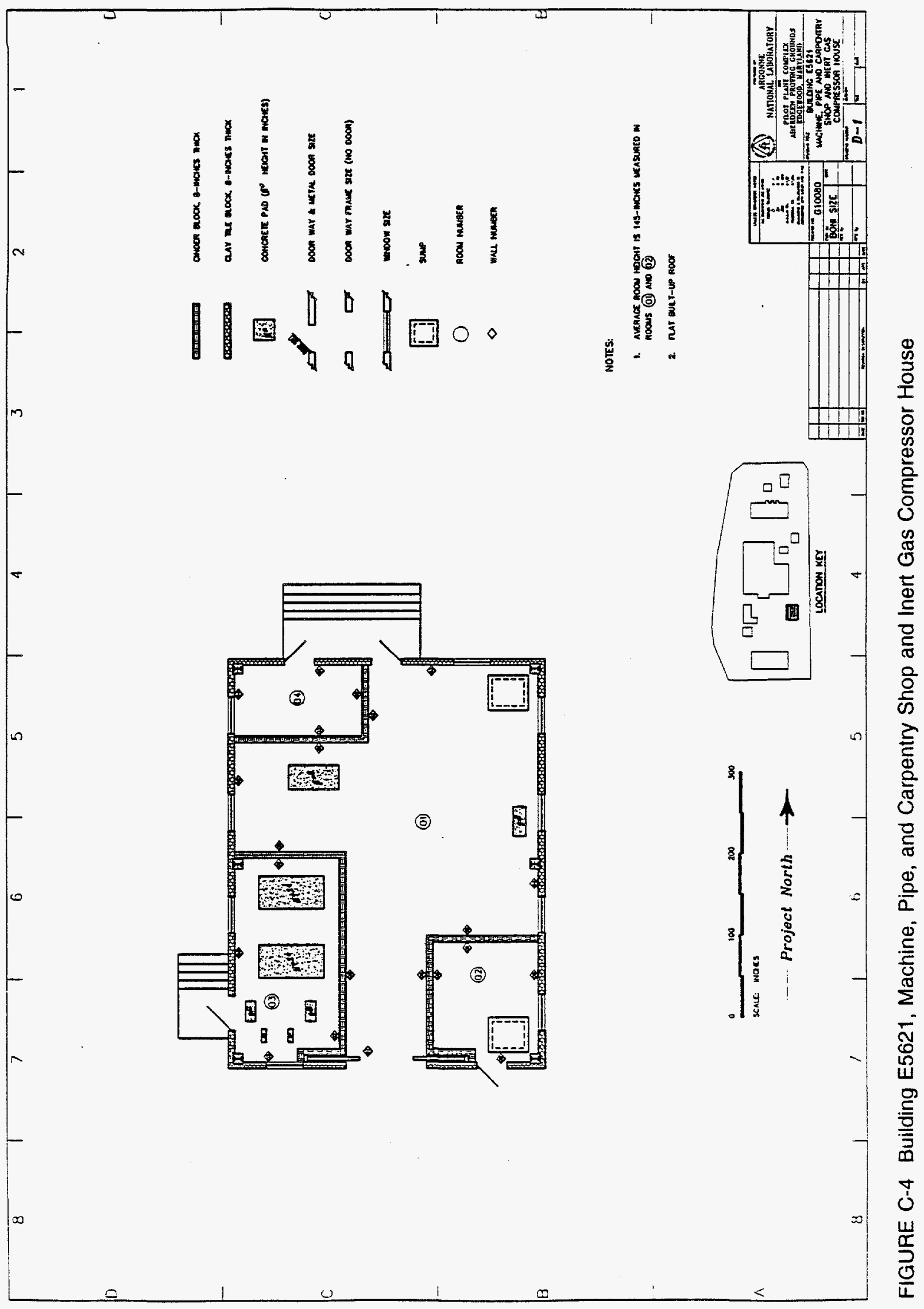




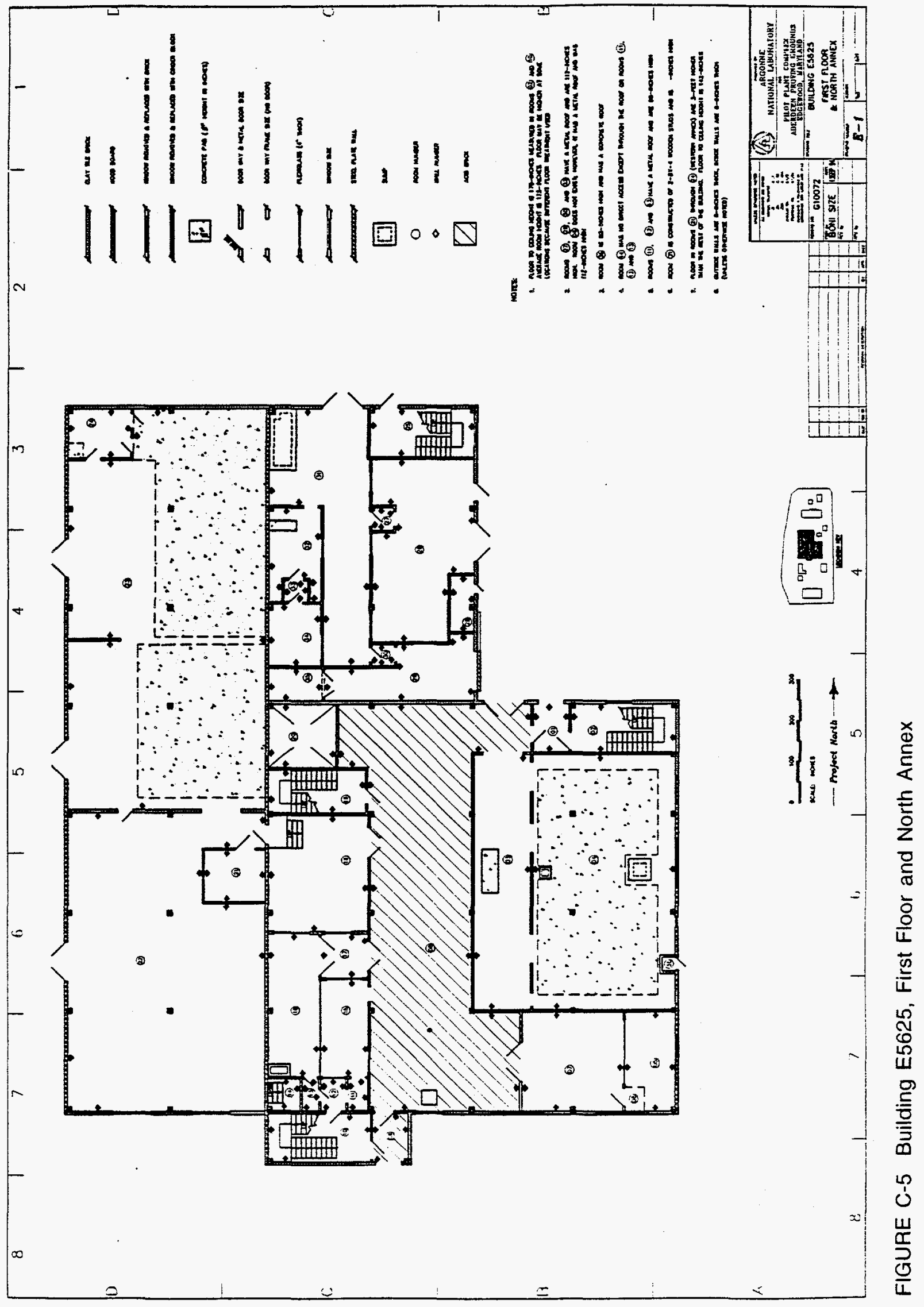




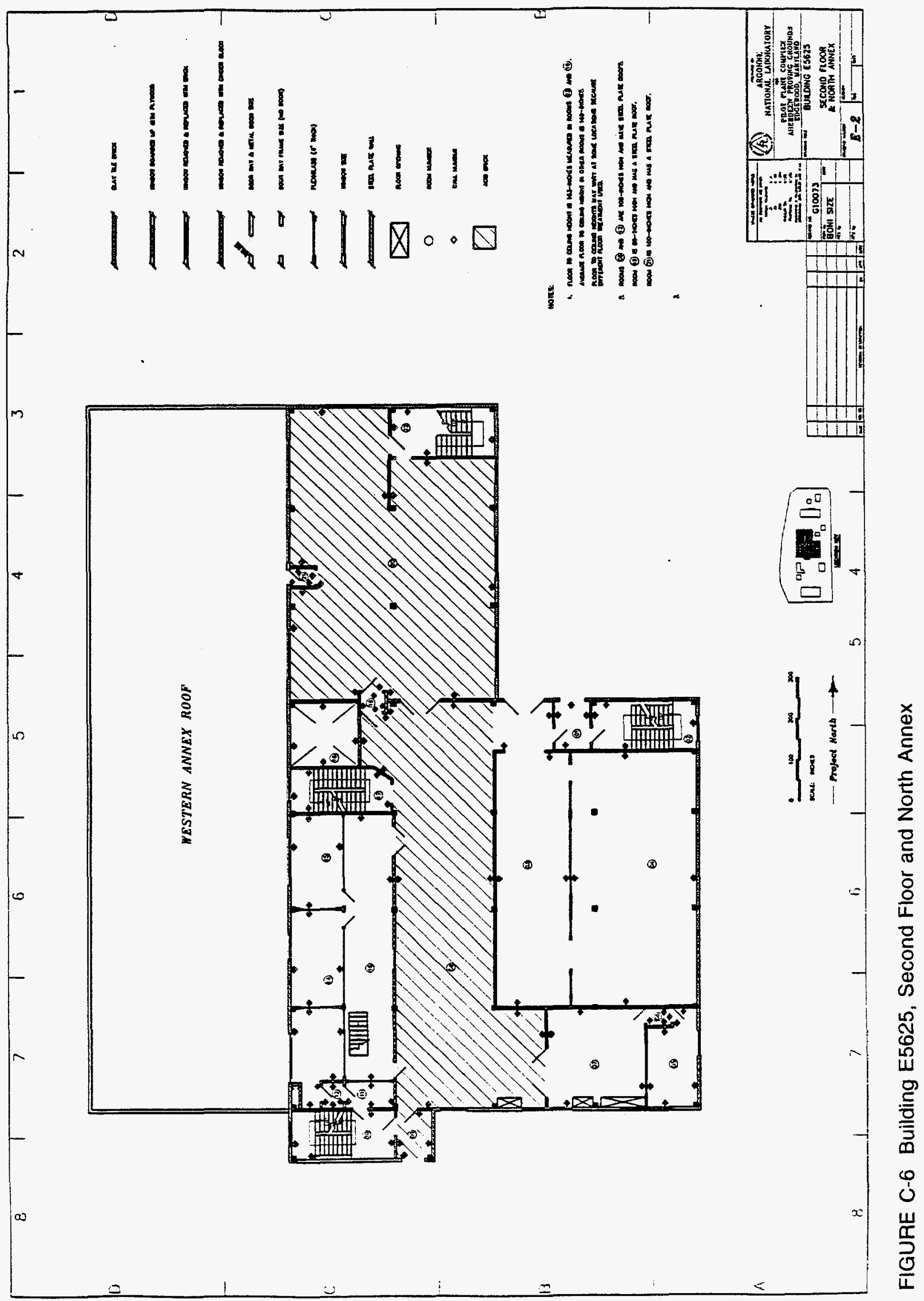




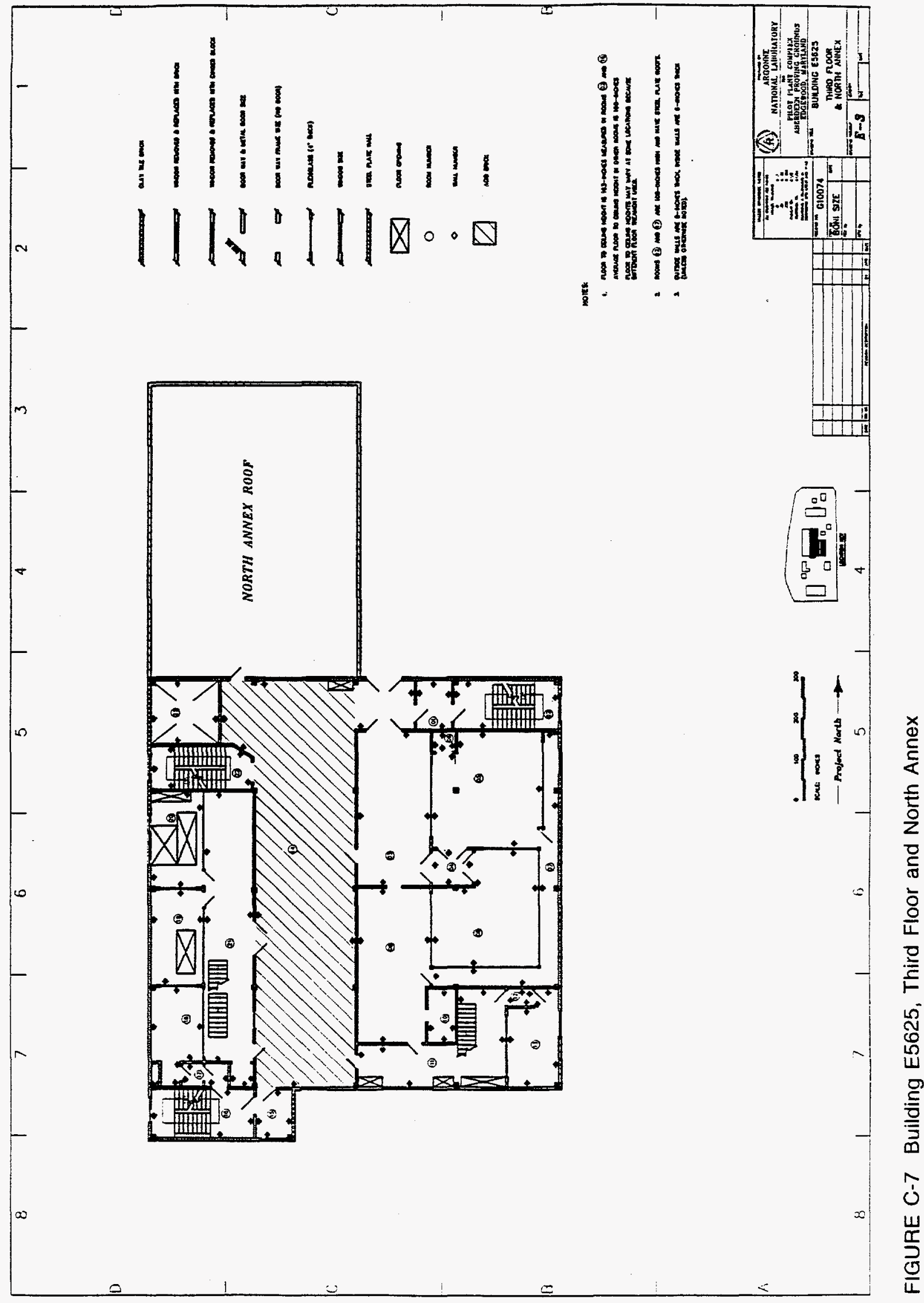




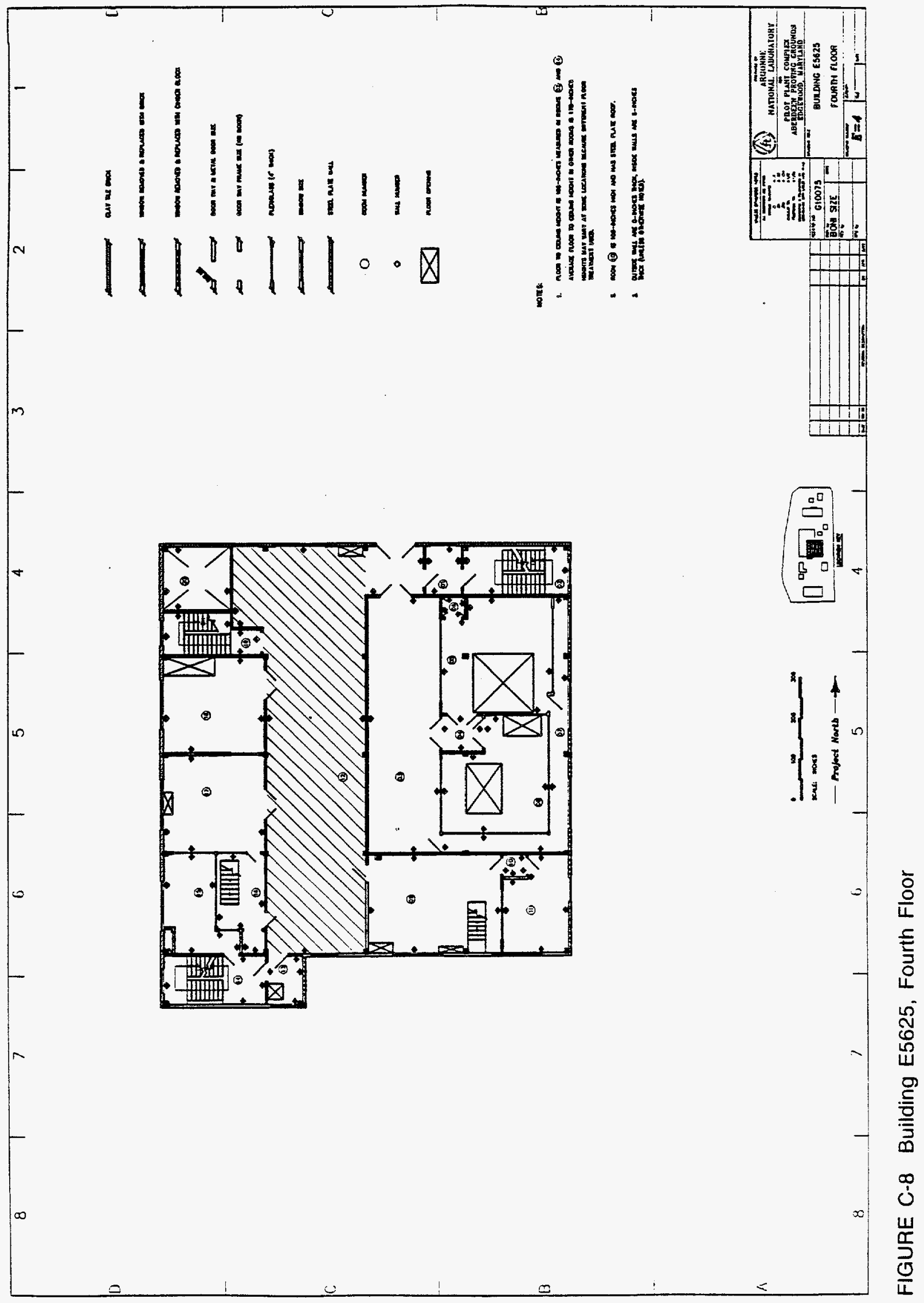




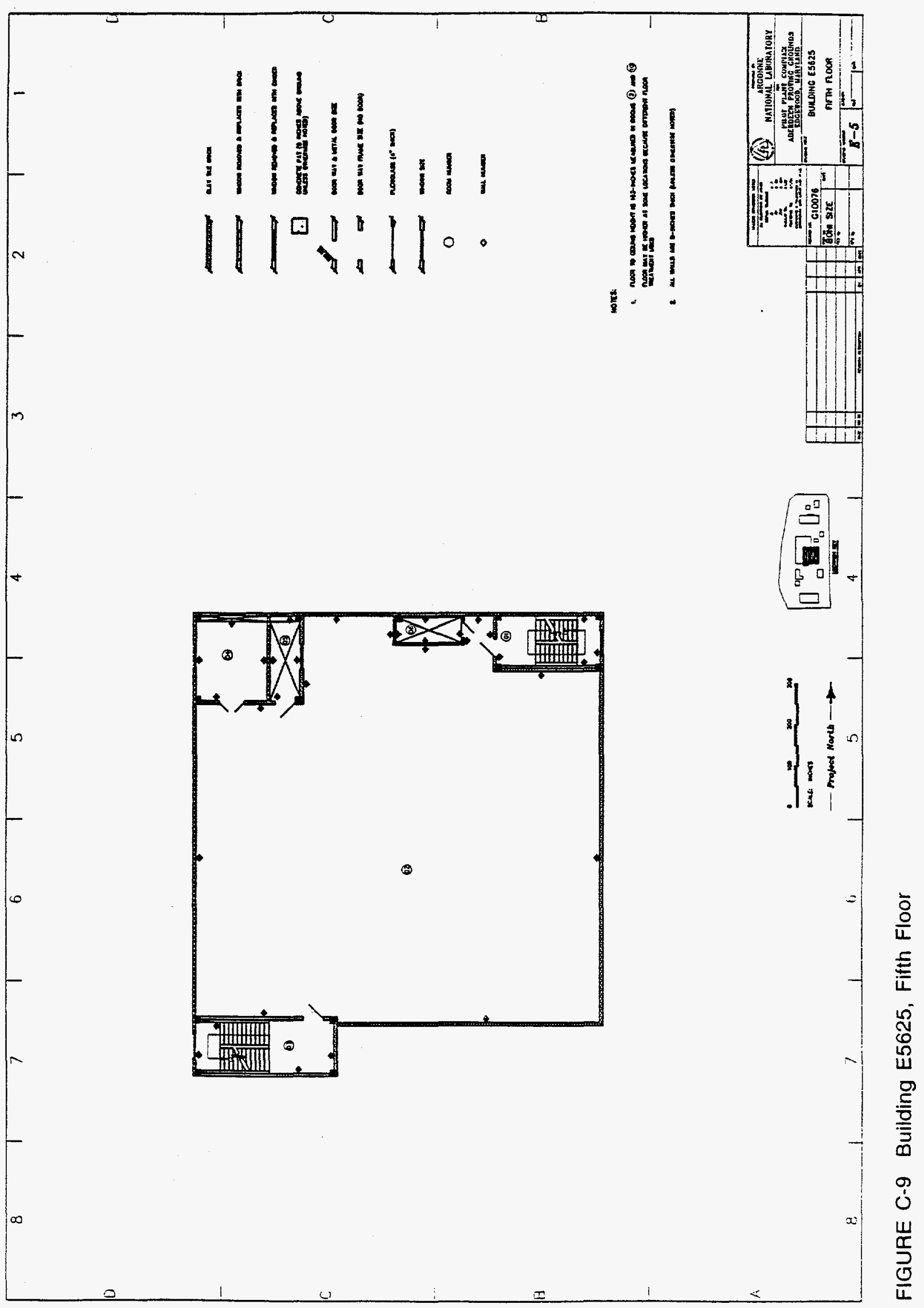




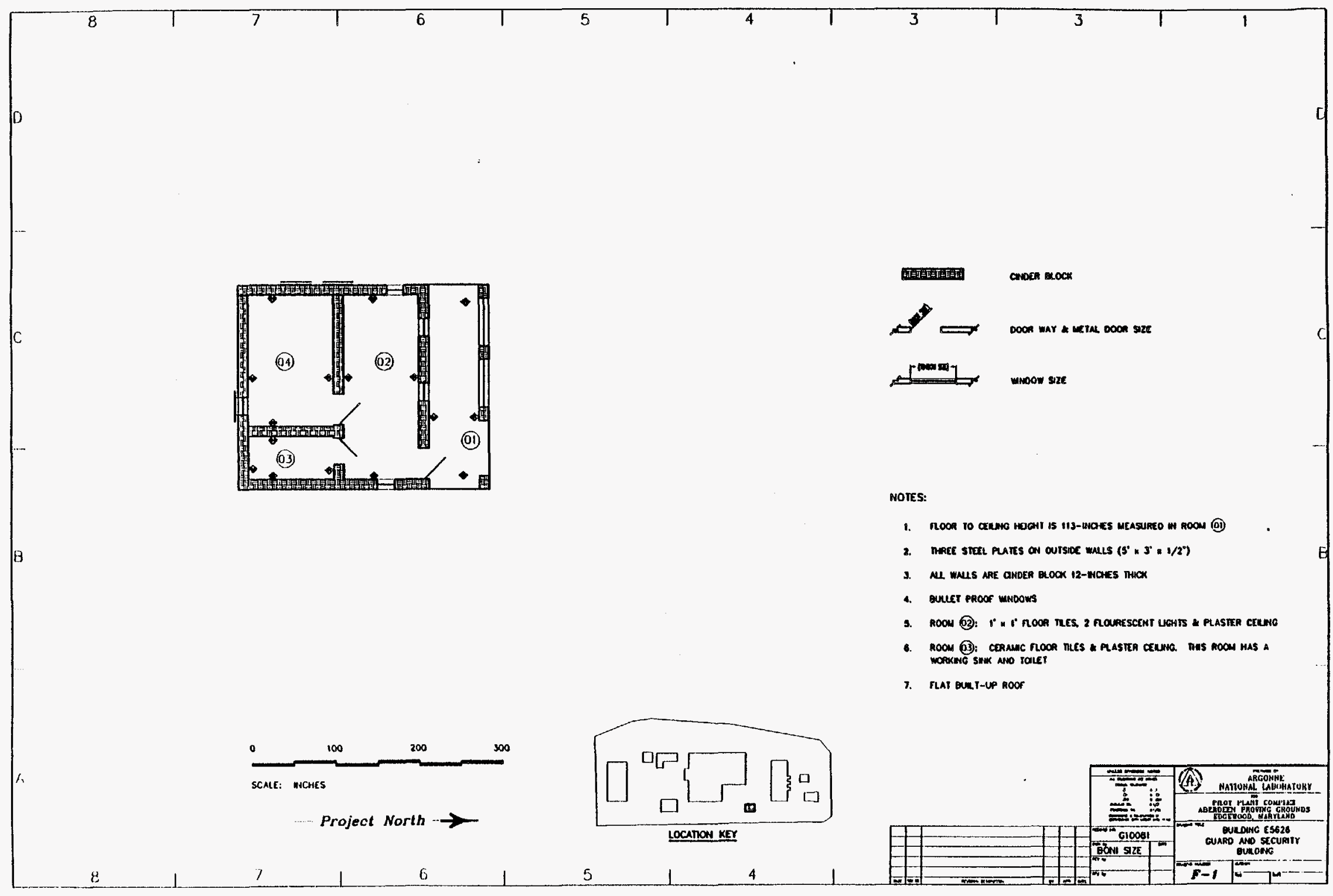

FIGURE C-10 Building E5626, Guard House and Security Building 


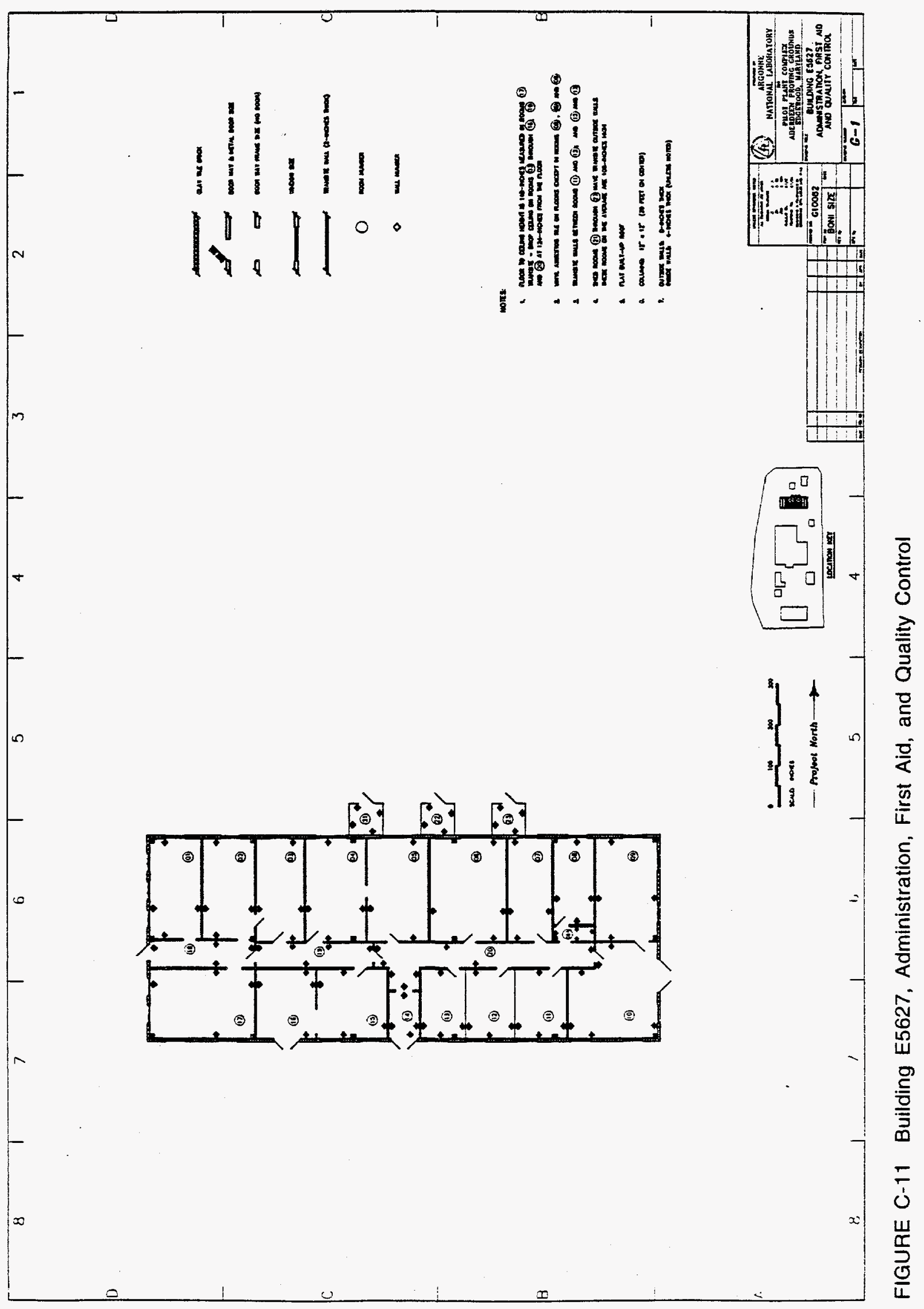




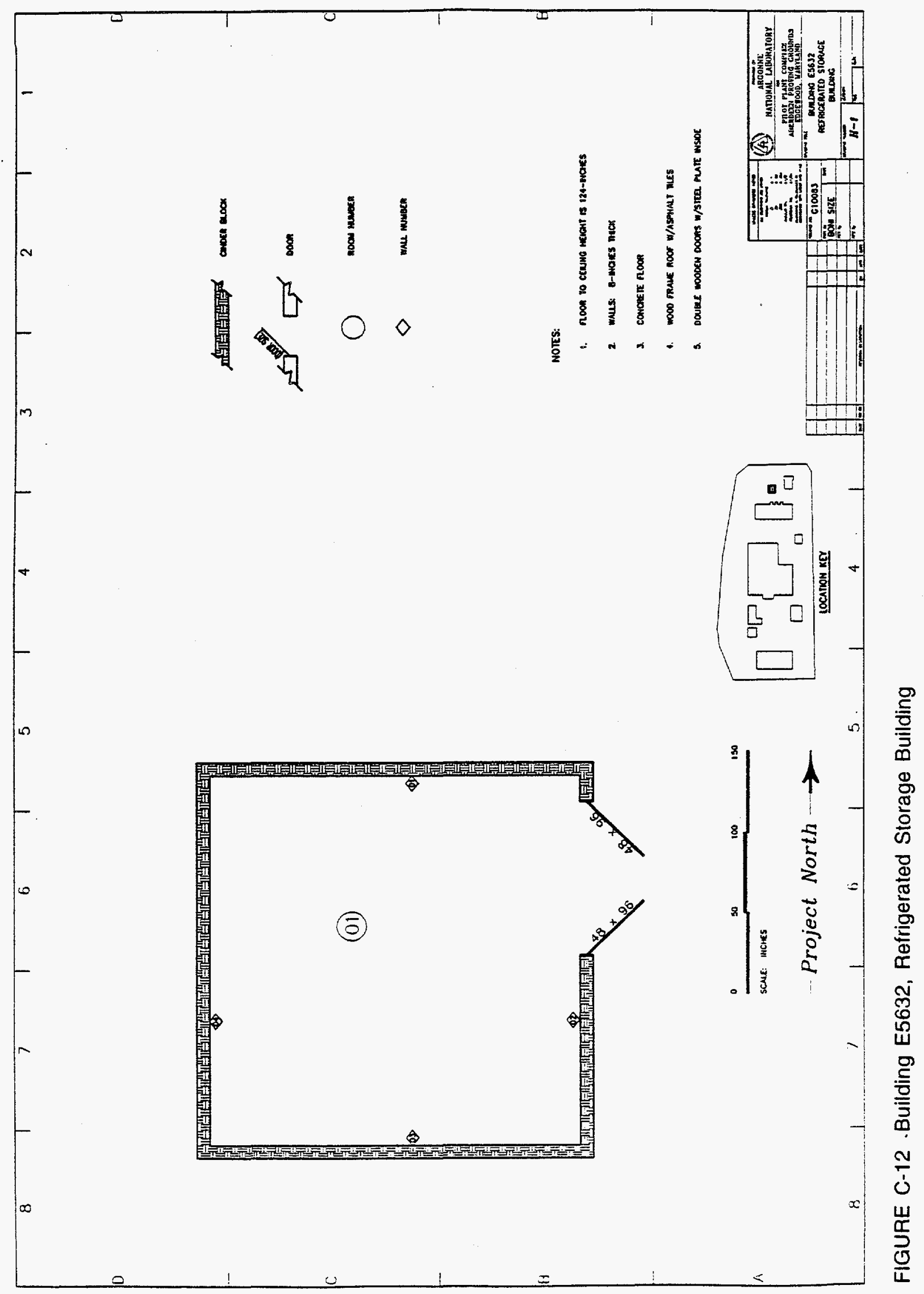




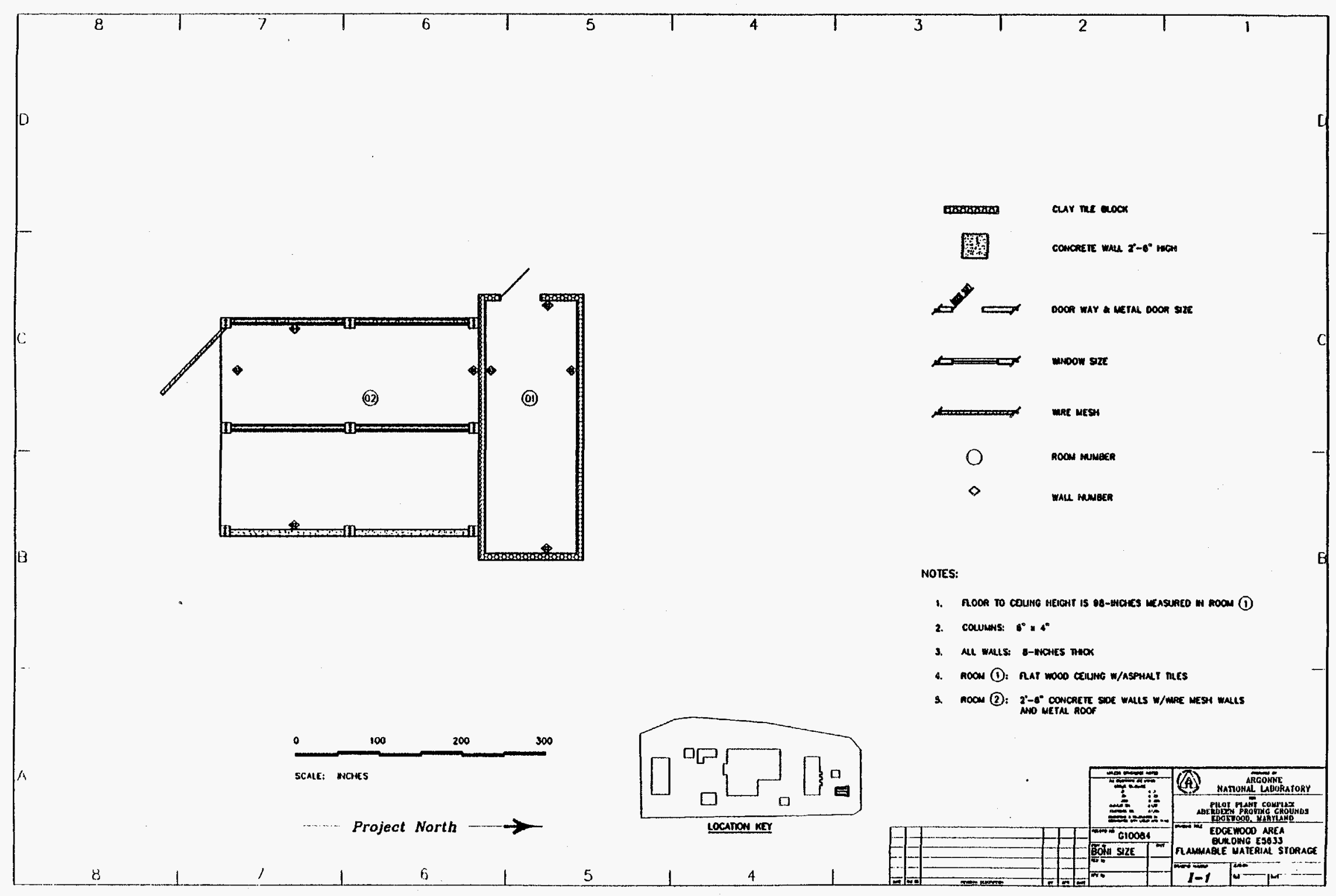

FIGURE C-13 Building E5633, Flammable Material Storage 
Appendix D:

Results of Analysis of Pilot Plant Complex Air Samples by Argonne National Laboratory 


\begin{tabular}{|c|c|c|c|c|c|c|c|c|c|c|c|c|c|c|c|c|}
\hline & & & & 116 & 118 & 114 & 115 & \begin{tabular}{|c|}
117 \\
107
\end{tabular} & 120 & \begin{tabular}{c|}
119 \\
1110
\end{tabular} & $\begin{array}{c}106 \\
101\end{array}$ & 107 & \begin{tabular}{c|}
93 \\
101
\end{tabular} & 173 & 100 & 167 \\
\hline & Compound & & & A107 & A107 & A107 & & & & & B101 & & & & & \\
\hline & Tentative Identification & RT & MW & ne & $n w$ & se & o. & sw & $n$ & $m$ & se & ㅁ & $m$ & m & ne & ne \\
\hline 1 & sulfur dioxide & 1.55 & 64 & & & & & & & & 0.09 & 0.14 & & & & \\
\hline 2 & 2-propanone & 1.89 & 58 & & & & & & & & & & & & & \\
\hline 3 & 1-fiuoro-1,1-dichloroethane & 1.95 & 116 & & & & & & & & & & & & & \\
\hline 4. & $\mathrm{~N}$-ethyl ethanamine & 2.36 & 73 & & & & & & & & & & & & & \\
\hline 5 & 2-butanone & 2.56 & 72 & & & & & & & & & & & & & \\
\hline 6 & $\mathrm{~N}$-ethyl-N-methyl ethanamine & 294 & 87 & & & & & & & & & & & & & \\
\hline 7 & dichloromethane & 3.15 & 84 & & & & & 0.15 & & & & & & & & \\
\hline 8 & $\mathrm{~N}$-(1-methylethyl)-2-propanamine & 3.59 & 101 & & & & & & & & & & & & & \\
\hline 9 & benzene & 3.64 & 78 & & 0.02 & 0.03 & 0.05 & & & 0.00 & 0.04 & 0.02 & & 0.22 & 0.02 & 0.53 \\
\hline 10 & 2-pentanone & 4.08 & 86 & & & & & & & & & & & & & \\
\hline 11 & trichloroethene & 4.47 & 130 & & & & & & & . & & & & 0.01 & & 0.02 \\
\hline 12 & N-(1-methylethylidene)-2-propanamine & 4.62 & 99 & & & & & & & & & & & & & \\
\hline 13 & toluene & 6.97 & 92 & 0.04 & 0.06 & 0.09 & 0.03 & 0.09 & 0.01 & 0.01 & 0.15 & 0.13 & 0.03 & 0.58 & 0.12 & 1.22 \\
\hline 14 & $\mathrm{~N}$-methyl-N-(1-methylethyl)-2-propanamine & 7.27 & 115 & & & & & & & & & & & & & \\
\hline 15 & 3-hexanone & 7.75 & 100 & & & & & & & & & & & & & \\
\hline 16 & 2-hexanone & 7.92 & 100 & & & & & & & & & & & & & \\
\hline 17 & n-octane & 8.29 & 114 & & & & & & & & & & & & & 0.13 \\
\hline 18 & hexanal & 8.33 & 100 & & & & & & & & & & & 0.59 & & \\
\hline 19 & tetrachloroethene & 8.79 & 164 & 0.00 & 0.00 & 0.01 & & & 0.00 & 0.01 & 0.02 & 0.01 & & & & \\
\hline 20 & methyl ethy disulfide & 9.68 & 108 & & & & & & & & & & & & & \\
\hline 21 & chlorobenzene & 10.05 & 112 & & & & & & & & & & & & & \\
\hline 22 & ethyl benzene & 10.57 & 106 & 0.01 & 0.01 & 0.02 & 0.01 & 0.02 & 0.00 & 0.01 & 0.03 & 0.03 & & 0.14 & 0.02 & 0.20 \\
\hline 23 & p,m-xydene & 10.81 & 106 & 0.02 & 0.02 & 0.06 & 0.03 & 0.07 & 0.01 & 0.01 & 0.07 & 0.06 & & 0.15 & 0.07 & 0.51 \\
\hline 24 & 1,4-oxathiane & 11.00 & 104 & & & & & & & & & & & & & \\
\hline 25 & styrene & 11.30 & 118 & & 0.01 & & & & & 0.00 & & & & & & \\
\hline 26 & 3-heptanone & 11.37 & 114 & & & & & & & & & & & & & \\
\hline 27 & o-xylene & 11.49 & 106 & & 0.01 & 0.01 & 0.01 & 0.03 & & 0.01 & 0.04 & 0.03 & & & 0.03 & 0.21 \\
\hline 28 & heptanal & 11.60 & 114 & & & & & & & & & & & & & \\
\hline 29 & n-nonane & 11.67 & 128 & & 0.02 & 0.03 & & 0.04 & & & 0.02 & & & & 0.01 & 0.45 \\
\hline 30 & C3-benzene & 12.20 & 120 & & & & & & & & & & & & & \\
\hline 31 & N-butylidene-1-butanamine & 12.28 & 127 & & & & & & & & & & & & & \\
\hline 32 & C3-benzene & 12.33 & 120 & & & & & & & & & & & & & \\
\hline 33 & 3-methyi nonane & 12.51 & 142 & & & & & & & & & & & & & \\
\hline 34 & pinene & 12.61 & 136 & & & & & & & & & & & & & \\
\hline 35 & 2-methyl cyclohexanol & 12.62 & 114 & & & & & & & & & & & & & \\
\hline 36 & 3-octanone & 12.71 & 128 & & & & & & & & & & & & & \\
\hline 37 & 2-ethyl hexanal & 13.02 & 128 & & & & & & & & & & & & & \\
\hline 38 & 1-chloro-2-methyl benzene & 13.03 & 126 & & & & & & & & & & & & & \\
\hline 39 & propyl benzene & 13.05 & 120 & & 0.01 & 0.02 & & & & & & 0.01 & & & & 0.13 \\
\hline 40 & N-butyl-1-butanamine & 13.17 & 129 & & & & & & & & & & & & & \\
\hline 41 & benzaldehyde & 13.18 & 106 & 0.02 & 0.03 & 0.04 & 0.44 & 0.09 & 0.02 & 0.02 & 0.04 & 0.04 & 0.06 & $0 . \overline{40}$ & 0.03 & 0.83 \\
\hline 42 & C3-benzene & 13.25 & 120 & & & & & & & & 0.03 & 0.03 & & & 0.04 & 0.31 \\
\hline 43 & C3-benzene & 13.41 & 120 & & & 0.04 & & & & & & & & & 0.05 & 0.08 \\
\hline 44 & phenol & 13.57 & 94 & 0.01 & 0.02 & & 0.06 & 0.06 & & 0.01 & 0.03 & & 0.02 & 0.72 & & 0.15 \\
\hline 45 & aniline & 13.61 & 93 & & & & & & & & & & & & & \\
\hline 46 & C3-benzene & 13.68 & 120 & & & & & & & & & & & & & \\
\hline 47 & 6-methyl-5-hepten-2-one & 13.69 & 126 & & & & & & & & & & & 0.11 & & \\
\hline 48 & benzonitrile & 13.72 & 103 & & & & 0.02 & & & & 0.03 & & & 0.15 & & \\
\hline 49 & 1-decene & 13.83 & 140 & & & & & & & & & & & & & \\
\hline 50 & C3-benzene & 13.99 & 120 & 0.02 & & 0.05 & & & & & & & & & & \\
\hline 51 & n-decane & 14.07 & 142 & 0.02 & 0.04 & 0.07 & & 0.08 & & 0.02 & 0.03 & 0.01 & & & 0.01 & \\
\hline 52 & octanal & 14.09 & 128 & & & & & & & & & & 0.01 & 0.29 & & \\
\hline 53 & dichlorobenzene & 14.40 & 146 & & & & & 0.02 & & & & & & & & \\
\hline 54 & 4-methyl decane & 14.56 & 156 & & & & & & & & & & & & & \\
\hline $55 !$ & C3-benzene & 14.57 & 120 & & & & & & & & & & & & & \\
\hline 56 & C4-benzene & 14.60 & 134 & & & & & & & & & & & & & \\
\hline 57 & 2-ethyl hexanol & 14.64 & 130 & & & 0.04 & & 0.03 & 0.23 & 0.28 & 0.09 & & & 0.37 & & \\
\hline 58 & limonene & 14.75 & 136 & & & & & & & & & & & 0.20 & 0.01 & \\
\hline 59 & benzene methanol & 14.77 & 108 & & & & & & & & & & & & & \\
\hline 60 & indan & 14.87 & 118 & & & & & & & & & & & & & \\
\hline 61 & dichlorobenzene & 14.89 & 146 & & & & & & & & & & & & & \\
\hline 62 & $\mathrm{~N}$-ethyl-N-(1-methylethy)-2-propanamine & 15.07 & 129 & & & & & & & & & & & & & \\
\hline 63 & C4-benzene & 15.10 & 134 & & & & & & & & & & & & & \\
\hline
\end{tabular}




\begin{tabular}{|c|c|c|c|c|c|c|c|c|c|c|c|c|c|c|c|c|}
\hline & & & & 116 & 118 & 114 & 115 & 117 & 120 & 119 & 106 & 107 & 93 & 173 & 100 & 167 \\
\hline & Compound & & & A107 & A107 & A107 & & A107 & Ato9 & A110 & 8101 & & C101 & C101 & C102 & $\mathrm{C} 102$ \\
\hline & Tentative Identification & RT & MW & ne & nw & se & 0 & sw & $n$ & $m$ & se & 0 & $m$ & $\mathrm{~m}$ & ne & ne \\
\hline 64 & 5-methyl decane & 15.21 & 156 & 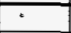 & & & & & & & & & & & & \\
\hline 65 & 4-methyl decane & 15.29 & 156 & & . & & & & & & & & & & & \\
\hline 66 & C4-benzene & 15.30 & 134 & & & & & & & & & & & & & \\
\hline 67 & 2-methyt decane & 15.34 & 156 & & & & & & & & & & & & & \\
\hline 68 & 1,4-dithiane & 15.39 & 120 & & & & & & & & & & & & & \\
\hline 69 & acetophenone & 15.47 & 120 & & 0.04 & 0.07 & 0.36 & 0.08 & & 0.03 & 0.03 & 0.03 & 0.05 & 0.35 & & 0.61 \\
\hline 70 & C4-benzene & 15.47 & 134 & & & & & & & & & & & & & 0.13 \\
\hline 71 & 3-methyl decane & 15.48 & 156 & & & & & & & & & & & & & \\
\hline 72 & methyl benzaldehyde & 15.51 & 120 & & & & & & & & & & & & & \\
\hline 73 & Ca-benzene & 15.64 & 134 & & & & & & & & & & & & & \\
\hline 74 & C4benzene & 15.72 & 134 & & & & & & & & & & & & & \\
\hline 75 & A,A-dimethy benzene methanol & 15.80 & 136 & & & & & & & & & & & & & \\
\hline 76 & C4-benzene & 15.86 & 134 & & & & & & & & & & & & & \\
\hline 77 & 1-undecene & 15.95 & 154 & & & & & & & & & & & & & \\
\hline 78 & n-undecane & 16.05 & 156 & 0.01 & 0.02 & 0.03 & & 0.03 & 0.01 & 0.01 & 0.01 & & & & & 0.11 \\
\hline 79 & nonanal & 16.12 & 142 & 0.02 & 0.04 & 0.05 & & 0.06 & 0.02 & 0.02 & 0.04 & 0.02 & 0.02 & 0.59 & & 0.31 \\
\hline 80 & C4-benzene & 16.24 & 134 & & & & & & & & & & & & & \\
\hline 81 & phosphoric acid, triethyl ester & 16.41 & 182 & & & & & & & & & & & & & \\
\hline 82 & C4-benzene & 16.43 & 134 & & & & & & & & & & & & & \\
\hline B3 & trans methy decalin & 96.50 & 152 & & & & & & & & & & & & & \\
\hline 84 & C4-benzene & 16.55 & 134 & & & & & & & & & & & & & \\
\hline 85 & cis methyl decalin & 16.74 & 152 & & & & & & & & & & & & & \\
\hline 86 & C5-benzene & 16.79 & 148 & & & & & & & & & & & & & \\
\hline 87 & methyl indan & 17.14 & 132 & & & & & & & & & & & & & \\
\hline 88 & 0,0 -diethyl-S-ethy/ phosphorothioate & 17.16 & 198 & & & & & & & & & & & & & \\
\hline 89 & methyl indan & 17.29 & 132 & & & & & & & & & & & & & \\
\hline 90 & trichlorobenzene & 17.64 & 180 & & & & & & & & & & & & & \\
\hline 91) & 1-dodecene & 17.66 & 168 & & & & & & & & & & & & & \\
\hline 92 & 1-(4-methylpheny) ethanone & 17.67 & 134 & & & & & & & & & & & & & \\
\hline 93 & $N, N$-dibutyl-1-butanamine & 17.72 & 442 & & & & & & & & & & & & & \\
\hline 94 & n-dodecane & 17.80 & 470 & & & & & & & & & & & 0.05 & & \\
\hline 95 & naphthalene & 17.79 & 128 & 0.05 & 0.10 & 0.48 & 0.01 & 0.19 & 0.01 & 0.04 & 0.08 & 0.01 & 0.76 & 0.40 & 0.69 & 2.06 \\
\hline 96 & C5-benzene & 17.87 & 148 & & & & & & & & & & & & & \\
\hline 97 & decanal & 17.90 & 156 & & 0.03 & 0.03 & & 0.04 & 0.01 & 0.04 & 0.03 & 0.02 & 0.03 & 0.48 & & 0.42 \\
\hline 98 & benzothiophene & 17.90 & 134 & & & & & & & & & & & & & 0.08 \\
\hline 99 & 1,4-oxathiane, 4,4-dioxide & 18.35 & 136 & & & & & & & & & & & & & \\
\hline 100 & benzothiazole & 18.40 & 135 & & & & & & & & & & & 0.20 & & \\
\hline 101 & benzene propanenitrile & 18.53 & 131 & & & & & & & & & & & & & \\
\hline 102 & dimethyl indan & 18.80 & 146 & & & & & & & & & & & & & \\
\hline 103 & 3-tetradecene & 18.85 & 196 & & & & & & & & & & & & & \\
\hline 104 & $\mathrm{~N}$-butyl-N-nitroso N-butanamine & 18.91 & 158 & & & & & & & & & & & & & \\
\hline 105 & 1-tridecene & 19.26 & 182 & & & & & & & & & & & & & \\
\hline 106 & n-tridecane & 19.38 & 184 & & & & & & & & & & & 0.03 & & \\
\hline 107 & $\mathrm{~N}, \mathrm{~N}$-dibutyl formamide & 19.51 & 157 & & & & & & & & & & & & & \\
\hline 108 & 2 -methyl naphthalene & 19.61 & 142 & 0.01 & 0.02 & 0.06 & & 0.05 & & 0.01 & 0.01 & & 0.10 & 0.09 & 0.17 & 0.38 \\
\hline 109 & phthalate & 19.80 & 390 & & & & 0.01 & & 0.02 & 0.03 & & & & & & \\
\hline 110 & 1,3-isobenzofurandione & 19.81 & 148 & & & & & & & 0.02 & & & & 0.07 & & \\
\hline 111 & 1-methyl naphthalene & 19.91 & 142 & & & 0.03 & & 0.03 & & & & & 0.06 & 0.11 & & 0.21 \\
\hline 112 & 1-methy-4-(propylthio) benzene & 20.37 & 166 & & & & & & & & & & & & & \\
\hline 113 & 1-tetradecene & 20.76 & 196 & & & & & & & & & & & & & \\
\hline 114 & n-tetradecane & 20.86 & 198 & & & & & & & & 0.01 & & & 0.05 & & \\
\hline 115 & biphenyl & 20.87 & 154 & 0.01 & 0.02 & 0.03 & 0.00 & 0.04 & & 0.01 & 0.01 & 0.00 & & & 0.03 & 0.05 \\
\hline 116 & 1,1'-oxy bis(benzene) & 21.17 & 186 & & & & & & & & & & & & & \\
\hline 117 & 2,4,6-trichloroaniline & 21.20 & 195 & & 0.01 & 0.02 & & 0.02 & 0.01 & & & & & 0.03 & & \\
\hline 118 & C2-naphthalene & 21.28 & 156 & 0.00 & & & & & & & & & & & & \\
\hline 119 & C2-naphthalene & 21.51 & 156 & & 0.01 & 0.02 & & 0.04 & & & & & & & 0.04 & 0.12 \\
\hline 120 & C2-naphthalene & 21.56 & 156 & & & & & & & & & & & & & \\
\hline 121 & tribromobenzene & 21.62 & 312 & & & & & & & & & & & & & \\
\hline 122 & 6, 10-dimethyl-5,9-undecadien-2-one & 21,65 & 194 & & & & & & & & & & & & & \\
\hline 123 & 2,6-di-t-butyl-2,5-cyclohexadiene-1,4-dione & 22.06 & 220 & & & & & & & & & & & & & \\
\hline 124 & 1-pentadecene & 22.16 & 210 & & & & & & & & & & & & & \\
\hline 125 & n-pentadecane & 22.26 & 212 & & & & & 0.02 & & & & & & & & \\
\hline 126 & methyl biphenyl & 22.30 & 168 & & & & & & & & & & & & & \\
\hline 127 & 1,2-dihydroacenaphthylene & 22.50 & 168 & & & & & & & & & & & & & \\
\hline north & $\begin{array}{l}\text { s south; e east; w west; } m \\
\text { tention time; MW molecular }\end{array}$ & ${ }^{6 / n}$ & I & 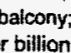 & 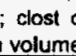 & & & & & & & & & & & \\
\hline
\end{tabular}




\section{Air Monitoring Results}

\begin{tabular}{|c|c|c|c|c|c|c|c|c|c|c|c|c|c|c|c|c|}
\hline & & & & 116 & 118 & 114 & 115 & 117 & 120 & 119 & 106 & 107 & 93 & 173 & 100 & 167 \\
\hline & Compound & & & A107 & A107 & A107 & & A107 & A109 & A110 & B101 & & C101 & \begin{tabular}{|l|} 
C101 \\
\end{tabular} & C102 & $\mathrm{C102}$ \\
\hline & Tentative Identification & RT & MW & ne & nw & se & 0 & sw & $n$ & $m$ & se & 으 & m & $m$ & ne & ne \\
\hline 128 & C3-naphthalene & 22.65 & 170 & & & & & & & & & & & & & \\
\hline 129 & phenyl maleic anhydride & 22.90 & 214 & & & & 0.02 & & & & & & & 0.17 & & 0.08 \\
\hline 130 & dibenzofuran & 22.93 & 168 & 0.01 & 0.02 & 0.03 & & 0.03 & & 0.01 & & & & & 0.01 & 0.06 \\
\hline 131 & 1-hexadecene & 22.47 & 224 & & & & & & & & & & & & & \\
\hline 132 & n-hexadecane & 23.57 & 226 & & & & & & & & & & & & & 0.04 \\
\hline 133 & benzenedicarboxylic acid, diethyi ester & 23.64 & 222 & & & & & & & & & & & & & \\
\hline 134 & 1,3-dibromo-2,2-bis(bromomethyl) propane & 24.17 & 384 & & & & & & & & & & & & & \\
\hline 135 & diphenyl diazene & 24.23 & 182 & & & & & & & & & & & & & \\
\hline 136 & benzophenone & 24.30 & 182 & & & & 0.03 & & & & & & & 0.05 & & \\
\hline 137 & 1-heptadecene & 24.71 & 238 & & & & & & & & & & & $\therefore$ & & \\
\hline 138 & n-heptadecane & 24.81 & 240 & & & & & & & & & & & & 0.01 & 0.13 \\
\hline 139 & $N$-(phenylmethytene) benzarnine & 24.83 & 181 & & & & & & & & & & & & & \\
\hline 140 & 9H-fluoren-9-one & 25.28 & 180 & & 0.02 & 0.03 & & 0.04 & & & & & & & & \\
\hline 141 & tribromobenzamine & 25.39 & 327 & & & & & & & & & & & & & \\
\hline 142 & trimethyl indan & 25.45 & 160 & & & & & & & & & & & 0.08 & & \\
\hline 143 & dibenzothiophene & 25.58 & 184 & & & & & & & & & & & & & \\
\hline 144 & tetrabromobenzene. & 25.86 & 390 & & & & & & & & & & & & & \\
\hline 145 & n-octadecane & 25.88 & 254 & & & & & & & & & & & & & \\
\hline 146 & phenanthrene/anthracene & 26.37 & 178 & 0.01 & 0.02 & 0.04 & & 0.05 & & 0.01 & 0.02 & & & & 0.03 & 0.26 \\
\hline 147 & methyl dibenzothiophene & 26.98 & 198 & & & & & & & & & & & & & \\
\hline 148 & n-nonadecane & 27.04 & 268 & & & & & & & & & & & & & \\
\hline 149 & 2-methyl anthracene/phenanthrene & 27.48 & 192 & & & & & & & & & & & & & \\
\hline 150 & 1-methyl anthracene/phenanthrene & 27.57 & 192 & & & & & & & & & & & & & \\
\hline 151 & 2,6-dibutyl-2,5-cyclohexadien-1,4-dione & 31.30 & 220 & & & & & & & & & 0.03 & & 0.50 & & 0.18 \\
\hline 152 & Total PCB's & & & & & 0.30 & & 0.40 & & & 0.50 & & 1.20 & 18.80 & 1.80 & 1.90 \\
\hline & & & & & & & & & & & & & & & & \\
\hline & & & & & & & & & & & & & & & & \\
\hline & & & & & & & & & & & & & & & & \\
\hline & & & & & & & & & & & & & & & & \\
\hline & & & & & & & & & & & & & & & & \\
\hline & & & & & & & & & & & & & & & & \\
\hline & & & & & & & & & & & & & & & & \\
\hline & & & & & & & & & & & & & & & & \\
\hline & & & & & & & & & & & & & & & & \\
\hline & & & & & & & & & & & & & & & & \\
\hline & & & & & & & & & & & & & & & & \\
\hline & & & & & & & & & & & & & & & & \\
\hline & & & & & & & & & & & & & & & & \\
\hline & & & & & & & & & & & & & & & & \\
\hline & & & & & & & & & & & & & & & & \\
\hline & & & & & & & & & & & & & & & & \\
\hline & & & & & & & & & & & & & & & & \\
\hline & & & & & & & & & & & & & & & & \\
\hline & & & & & & & & & & & & & & & & \\
\hline & & & & & & & & & & & & & & & & \\
\hline & & & & & & & & & & & & & & & & \\
\hline & & & & & & & & & & & & & & & & \\
\hline & & & & & & & & & & & & & & & & \\
\hline & & & & & & & & & & & & & & & & \\
\hline & & & & & & & & & & & & & & & & \\
\hline & & & & & & & & & & & & & & & & \\
\hline & & & & & & & & & & & & & & & & \\
\hline & & & & & & & & & & & & & & & & \\
\hline & & & & & & & & & & & & & & & & \\
\hline & & & & & & & & & & & & & & & & \\
\hline & & & & & & & & & & & & & & & & \\
\hline & & & & & & & & & & & & & & & & \\
\hline & & & & & & & & & & & & & & & & \\
\hline & & & & & & & & & & & & & & & & \\
\hline & & & & & & & & & & & & & & & & \\
\hline & & & & & & & & & & & & & & & & \\
\hline & & & & & & & & & & & & & & & & \\
\hline & 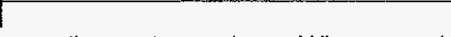 & . & 1 & 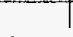 & 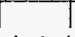 & 1 & 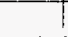 & & & & & & & & & \\
\hline
\end{tabular}

Sample number and room number at the top of each data column 


\begin{tabular}{|c|c|c|c|c|c|c|c|c|c|c|c|c|c|c|c|c|}
\hline & Compound & & & \begin{tabular}{|c|}
101 \\
$C 102$ \\
\end{tabular} & \begin{tabular}{|c|}
168 \\
$C 102$ \\
\end{tabular} & \begin{tabular}{|c|}
102 \\
C103 \\
\end{tabular} & \begin{tabular}{|c|}
169 \\
$C 103$ \\
\end{tabular} & \begin{tabular}{|c|}
170 \\
$\mathrm{C} 103$ \\
\end{tabular} & \begin{tabular}{|c|}
103 \\
C103 \\
\end{tabular} & \begin{tabular}{|c|}
104 \\
$C 104$ \\
\end{tabular} & \begin{tabular}{|c|}
105 \\
C104 \\
\end{tabular} & \begin{tabular}{|c|}
171 \\
C104 \\
\end{tabular} & \begin{tabular}{|c|}
172 \\
$C 104$ \\
\end{tabular} & \begin{tabular}{|c|}
109 \\
0101 \\
\end{tabular} & \begin{tabular}{|c|}
112 \\
0101 \\
\end{tabular} & \begin{tabular}{|c|}
108 \\
0101 \\
\end{tabular} \\
\hline & Tentative Identification & RT & MW & sw & sw & ne & ne & sw & Sw & ne & sw & ne & sw & e & $w$ & ne \\
\hline 1 & sulfur dioxide & 1.55 & 64 & & & & & & & & & & & & & \\
\hline 2 & 2-propanone & 1.89 & 58 & & & & & & & & & & & & & \\
\hline 3 & 1-fluoro-1,1-dichloroethane & 1.95 & 116 & & & & & & & & & & & & & \\
\hline 4 & N-ethyl ethanamine & 2.36 & 73 & & & & & & & & & & & & & \\
\hline 5 & 2-butanone & 2.56 & 72 & & & & & & & & & & & & & \\
\hline 6 & $\mathrm{~N}$-ethyl-N-methyl ethanamine & 2.94 & 87 & & & & & & & & & & & & & \\
\hline 7 & dichloromethane & 3.15 & 84 & & & & & & & & 0.23 & & & & & \\
\hline 8 & $\mathrm{~N}$-(1-methylethyl)-2-propanamine & 3.59 & 101 & & & & & & & & & & & & & \\
\hline 9 & benzene & 3.64 & 78 & & 0.09 & 0.03 & 0.08 & 0.36 & 0.02 & 0.01 & 0.03 & 0.26 & 0.48 & 0.03 & & 0.01 \\
\hline 10 & 2-pentanone & 4.08 & 86 & & & & & & & & & & & & & \\
\hline 11 & trichloroethene & 4.47 & 130 & & & & & $t$ & & & & 0.01 & 0.01 & & & \\
\hline 12 & $\mathrm{~N}-(1-$ methylethylidene $)-2$-propanamine & 4.62 & 99 & & & & & & & & & & & & & \\
\hline 13 & toluene & 6.97 & 92 & 0.07 & 0.10 & 0.13 & & 0.60 & 0.09 & 0.07 & 0.15 & 1.06 & 0.89 & 0.13 & 0.11 & 0.08 \\
\hline 14 & N-methyl-N-(1-methylethyi)-2-propanamine & 7.27 & 115 & & & & & & & & & & & & & \\
\hline 15 & 3-hexanone & 7.75 & 100 & & & & & & & & & & & & & \\
\hline 16 & 2-hexanone & 7.92 & 100 & & & & & & & & & & & & & \\
\hline 17 & n-octane & 8.29 & 114 & & & & & 0.27 & & & & & & & & \\
\hline 18 & hexanal & 8.33 & 100 & & 0.14 & & 0.13 & & & & & & 2.91 & & & \\
\hline 19 & tetrachloroethene & 8.79 & 164 & & & 0.02 & & & 0.02 & 0.01 & 0.02 & & & 0.01 & & 0.01 \\
\hline 20 & methyl ethyi disulfide & 9.68 & 108 & & & & & & & & & & & & & \\
\hline 21 & chlorobenzene & 10.05 & 112 & & & & & & & & & & & & & \\
\hline 22 & ethyl benzene & 10.57 & 106 & 0.02 & $\mathrm{t}$ & & $t$ & 0.19 & 0.02 & 0.02 & 0.03 & 0.15 & 0.34 & 0.01 & 0.03 & 0.02 \\
\hline 23 & $p, m-x y$ lene & 10.81 & 106 & 0.07 & $t$ & 0.05 & $t$ & 0.22 & 0.04 & 0.04 & 0.07 & 0.31 & 0.29 & 0.09 & 0.11 & 0.07 \\
\hline 24 & 1,4-oxathiane & 11.00 & 104 & & & & & & & & & & & & & \\
\hline 25 & styrene & 11.30 & 118 & & & & & & 0.01 & & & & & & 0.02 & 0.01 \\
\hline 26 & 3-heptanone & \begin{tabular}{|l|l|}
11.37 \\
\end{tabular} & 114 & & & & & & & & & & & & & \\
\hline 27 & o-xylene & \begin{tabular}{|c|}
11.49 \\
\end{tabular} & 106 & 0.04 & $t$ & 0.02 & & & 0.02 & 0.02 & 0.03 & 0.26 & & & 0.04 & 0.02 \\
\hline 28 & heptanal & 11.60 & 114 & & & & & & & & & & 0.41 & & & \\
\hline 29 & n-nonane & \begin{tabular}{|l|l|}
11.67 \\
\end{tabular} & 128 & 0.01 & 0.13 & 0.01 & 0.07 & 0.35 & 0.01 & 0.01 & 0.02 & & & & & \\
\hline 30 & C3-benzene & 12.20 & 120 & & & & & & & & & & & & & \\
\hline 31 & $\mathrm{~N}$-butylidene-1-butanarnine & 12.28 & 127 & & & & & & & & & & & & & \\
\hline 32 & C3-benzene & 12.33 & 120 & & & & & & & & & & & & & \\
\hline 33 & 3-methyl nonane & 12.51 & 142 & & & & & & & & & & & & & \\
\hline 34 & pinene & 12.61 & 136 & & & & & & & & & 0.06 & & 0.32 & 0.26 & 0.18 \\
\hline 35. & 2-methyl cyclohexanol & \begin{tabular}{|l|l|}
12.62 \\
\end{tabular} & 114 & & & & & & & & & & & & & \\
\hline 36 & 3-octanone & 12.71 & 128 & & & & & & & & & & & & & \\
\hline 37 & 2-ethyl hexanal & 13.02 & 128 & & & & & & & & & & & & & \\
\hline 38 & 1-chloro-2-methyl benzene & 13.03 & 126 & & & & & & & & & & & & & \\
\hline 39 & propyl benzene & 13.05 & 120 & & & & & & & & 0.01 & & & & & \\
\hline 40 & $\mathrm{~N}$-butyl-1-butanamine & 13.17 & 129 & & & & & & & & & & & & & \\
\hline 41 & benzaldehyde & 13.18 & 106 & 0.03 & 0.20 & 0.03 & 0.20 & 0.60 & 0.03 & 0.02 & 0.02 & 0.70 & 2.00 & 0.03 & 0.06 & 0.03 \\
\hline 42 & C3-benzene & 13.25 & 120 & 0.04 & & 0.03 & & & & & 0.03 & & & & & 0.03 \\
\hline 43 & C3-benzene & 13.41 & 120 & 0.05 & & & & & & & & & & & & \\
\hline 44 & phenol & \begin{tabular}{|l|}
13.57 \\
\end{tabular} & 94 & & & & & 0.19 & & & & 0.15 & 0.25 & 0.04 & 0.04 & 0.01 \\
\hline 45 & aniline & 13.61 & 93 & & & & & & & & & & & & & \\
\hline 46 & C3-benzene & \begin{tabular}{|c|}
13.68 \\
\end{tabular} & 120 & & & & & & & & & & & & & \\
\hline 47 & 6-methy-5-hepten-2-one & 13.69 & 126 & & & & 0.06 & 0.08 & & & & 0.08 & & & & \\
\hline 48 & \begin{tabular}{|l} 
benzonitrile \\
\end{tabular} & 13.72 & 103 & & 0.03 & & & & & & & 0.16 & 0.20 & & & \\
\hline 49 & 1-decene & 13.83 & 140 & & & & & & & & & & & & & \\
\hline 50 & C3-benzene & \begin{tabular}{|l|}
13.99 \\
\end{tabular} & 120 & & & & & & & & & 0.10 & & & & \\
\hline 51 & n-decane & \begin{tabular}{|l|l|}
14.07 \\
\end{tabular} & 142 & & & & & & & & & & & & & \\
\hline 52 & octanal & 14.09 & 128 & & 0.11 & & 0.18 & 0.40 & & & & 0.05 & 0.40 & & & \\
\hline 53. & dichlorobenzene & 14.40 & 146 & & & & & & & & & & & & & \\
\hline 54 & 4-methyl decane & 14.56 & 156 & & & & & & & & & & & & & \\
\hline 55 & C3-benzene & 14.57 & 120 & & & & & & & & & & & & & \\
\hline 56 & C4-benzene & 14.60 & 134 & 0.03 & & & & & & & & & & & & \\
\hline 57 & 2-ethyl hexanol & 14.64 & 130 & & 0.06 & 0.02 & 0.11 & 0.09 & & & & 0.09 & 0.58 & 0.06 & 0.13 & 0.11 \\
\hline 58 & limonene & \begin{tabular}{|l|l|}
14.75 \\
\end{tabular} & 136 & & 0.04 & 0.01 & & & & & 0.02 & & & 0.05 & 0.07 & 0.05 \\
\hline 59 & benzene methanol & 14.77 & 108 & & & & & & & & & & & & & \\
\hline 60 & indan & 14.87 & 118 & & & & & & & & & & & & & \\
\hline 61 & dichlorobenzene & 14.89 & 146 & & & & & & & & & & & & & \\
\hline 62 & N-ethyl-N-(1-methylethyl)-2-propanamine & $\mid 15.07$ & 129 & & & & & & & & & & & & & \\
\hline 63 & C4-benzene & $|15.10|$ & 134 & & & & & & & & & & & & & \\
\hline
\end{tabular}




\begin{tabular}{|c|c|c|c|c|c|c|c|c|c|c|c|c|c|c|c|c|}
\hline & & & & 101 & 168 & 102 & 169 & 170 & 103 & 104 & 105 & 171 & 172 & 109 & 112 & 108 \\
\hline & Compound & & & $C_{102}$ & C102 & C103 & $\mathrm{C1O3}$ & C103 & C103 & C104 & C104 & C104 & C104 & D101 & D101 & D101 \\
\hline & Tentative Identification & RT & MW & sw & sw & ne & ne & sw & sw & ne & sw & ne & sw & $\mathrm{e}$ & $w$ & ne \\
\hline 64 & 5-methyl decane & 15.21 & 156 & & & & & & & & & & & & & \\
\hline 65 & 4-methyl decane & 15.29 & 156 & & & & & & & & & & & & & \\
\hline 66 & C4-benzene & 15.30 & 134 & & & & & & & & & & & & & \\
\hline 67 & 2-methyl decane & 15.34 & 156 & & & & & & & & & & & & & \\
\hline 68 & 1,4-dithiane & 15.39 & 120 & & & & & & & & & & & & & \\
\hline 69 & acelophenone & 15.47 & 120 & & 0.18 & & 0.11 & 0.32 & & & & 0.40 & 0.69 & & & \\
\hline 70 & C4-benzene & 15.47 & 134 & & & & & & & & & & & & & \\
\hline 71 & 3-methyl decane & 15.48 & 156 & & & & & & & & & & & & & \\
\hline 72 & methyl benzaldehyde & 15.51 & 120 & & & & & & & & & & & & & \\
\hline 73 & C4-benzene & 15.64 & 134 & & & & & & & & & & & & & \\
\hline 74 & C4-benzene & 15.72 & 134 & & & & & & & & & & & & & \\
\hline 75 & A,A-dimethyl benzene methanol & 15.80 & 136 & & & & & & & & & & & & & \\
\hline 76 & C4-benzene & 15.86 & 134 & & & & & & & & & & & & & \\
\hline 77 & 1-undecene & 15.95 & 154 & & & & & & & & & & & & & \\
\hline 78 & n-undecane & 16.05 & 156 & & & & & & & & & 0.08 & 0.06 & & & \\
\hline 79 & nonanal & 16.12 & 142 & & 0.30 & 0.03 & 0.52 & 0.92 & 0.04 & & 0.02 & 0.19 & 0.87 & 0.04 & 0.07 & \\
\hline 80 & C4-benzene & 16.24 & 134 & & & & & & & & & & & & & \\
\hline 81 & phosphoric acid, triethyl ester & 16.41 & 182 & & & & & & & & & & & & & \\
\hline 82 & C4-benzene & 16.43 & 134 & & & & & & & & & & & & & \\
\hline 83 & trans methyl decalin & 16.50 & 152 & & & & & & & & & & & & & \\
\hline 84 & C4-benzene & 16.55 & 134 & & & & & & & & & & & & & \\
\hline 85 & cis methyl decalin & 16.74 & 152 & & & & & & & & & & & & & \\
\hline 86 & C5-benzene & 16.79 & 148 & & & & & & & & & & & & & \\
\hline 87 & methyl indan & 17.14 & 132 & & & & & & & & & & & & & \\
\hline 88 & 0,0 -diethyl-s-ethyl phosphorothioate & 17.16 & 198 & & & & & & & & & & & & & \\
\hline 89 & methyl indan & 17.29 & 132 & & & & & & & & & & & & & \\
\hline 90 & trichlorobenzene & 17.64 & 180 & & & & & & & & & & & & & \\
\hline 91 & 1-dodecene & 17.66 & 168 & & & & & & & & & & & & & \\
\hline 92 & 1-(4-methylphenyl) ethanone & 17.67 & 134 & & & & & & & & & & & & & \\
\hline 93 & N,N-dibutyl-1-butanamine & 17.72 & 142 & & & & & & & & & & & & & \\
\hline 94 & n-dodecane & 17.80 & 170 & & & & & & & & & & & & & \\
\hline 95 & naphthalene & 17.79 & 128 & 0.81 & 0.04 & 0.68 & 0.10 & 1.76 & 0.43 & 0.16 & 0.32 & 1.43 & 0.89 & 0.26 & 0.68 & 0.20 \\
\hline 96 & C5benzene & \begin{tabular}{|l|l|}
17.87 \\
\end{tabular} & 148 & & & & & & & & & & & & & \\
\hline 97 & decanal & 17.90 & 156 & & 0.34 & 0.04 & 0.67 & 0.96 & 0.04 & & 0.03 & 0.17 & 0.67 & 0.03 & 0.06 & \\
\hline 98 & benzothiophene & 17.90 & 134 & 0.03 & & & & 0.07 & & & & & & & & \\
\hline 99 & 1,4-oxathiane, 4,4-dioxide & 18.35 & 136 & & & & & & & & & & & & & \\
\hline 100 & benzothiazole & 18.40 & 135 & & & & & & 0.01 & & & & & & & \\
\hline 101 & benzene propanenitrile & 18.53 & 131 & & & & & & & & & & & & & \\
\hline 102 & dimethyl indan & 18.80 & 146 & & & & & & & & & & & & & \\
\hline 103 & 3-tetradecene & 18.85 & 196 & & & & & & & & & & & & & \\
\hline 104 & $\mathrm{~N}$-butyl-N-nitroso N-butanamine & 18.91 & 158 & & & & & & & & & & & & & \\
\hline 105. & 1-tridecene & 19.26 & 182 & & & & & & & & & & & & & \\
\hline 106 & n-tridecane & 19.38 & 184 & & & & & & & & & & & & & \\
\hline 107 & N,N-dibutyl formamide & 19.51 & 157 & & & & & & & & & & & & & \\
\hline 108 & 2-methyi naphthalene & 19.61 & 142 & 0.11 & & 0.09 & & 0.31 & 0.06 & 0.02 & 0.04 & 0.25 & 0.15 & 0.07 & 0.21 & 0.06 \\
\hline 109 & phthalate & 19.80 & 390 & & & & & & & & & & & & & \\
\hline 310 & 1,3-isobenzofurandione & 19.81 & 148 & & 0.03 & & & & & & & & 0.13 & & & \\
\hline 111 & 1 -methy naphthalene & 19.91 & 142 & 0.06 & & 0.05 & & 0.24 & & & 0.02 & 0.14 & 0.11 & 0.05 & 0.13 & 0.04 \\
\hline 112 & 1-methyl-4-(propylthio) benzene & 20.37 & 166 & & & & & & & & & & & & & \\
\hline 113 & 1-tetradecene & 20.76 & 196 & & & & & & & & & & & & & \\
\hline 114 & n-tetradecane & 20.86 & 198 & & & & & & & & & 0.10 & & & & \\
\hline 115 & biphenyl & 20.87 & 154 & 0.03 & & 0.03 & & 0.10 & 0.02 & 0.01 & 0.01 & 0.05 & 0.04 & 0.04 & 0.10 & 0.03 \\
\hline 116 & $1,1^{\prime}$-oxy bis(benzene & 21.17 & 186 & & & & & & & & & & & & & \\
\hline 117 & $2,4,6$-trichloroaniline & 21.20 & 195 & & & & & & & & & & & & & \\
\hline 118 & C2-naphthalene & 21.28 & 156 & & & & & & & & & & & & 0.07 & \\
\hline 119 & C2-naphthalene & 21.51 & 156 & 0.04 & & & & & 0.01 & & & 0.07 & & 0.08 & 0.04 & 0.01 \\
\hline 120 & C2-naphthalene & 21.56 & 156 & & & & & & & & & & & & & \\
\hline 121 & tribromobenzene & 21.62 & 312 & & & & & & & & & & & & & \\
\hline 122 & 6,10-dimethyl-5,9-undecadien-2-one & 21.65 & 194 & & & & & & & & & & & & & \\
\hline 123 & 2,6-di-t-butyl-2,5-cyclohexadiene-1,4-dione & 22.06 & 220 & & & & & & & & & & & & & \\
\hline 124 & 1-pentadecene & 22.16 & 210 & & & & & & & & & & & & & \\
\hline 125 & n-pentadecane & 22.26 & 212 & & & 0.01 & & & & & & 0.03 & & & & \\
\hline 126 & methyi bipheny & 22.30 & 168 & & & & & & & & & & & 0.01 & & \\
\hline 127 & 1,2-dihydroacenaphthylene & 22.50 & 168 & & & & & & & & & & & & 0.04 & \\
\hline
\end{tabular}


Air Monitoring Results

Sample number and room number at the top of each data column

\begin{tabular}{|c|c|c|c|c|c|c|c|c|c|c|c|c|c|c|c|c|}
\hline & Compound & & & \begin{tabular}{|c|}
101 \\
C102 \\
\end{tabular} & \begin{tabular}{|c|}
168 \\
$C 102$ \\
\end{tabular} & \begin{tabular}{|c|}
102 \\
C103 \\
\end{tabular} & \begin{tabular}{|c|}
169 \\
$C 103$ \\
\end{tabular} & \begin{tabular}{|c|}
170 \\
C103 \\
\end{tabular} & \begin{tabular}{|c|}
103 \\
$C 103$ \\
\end{tabular} & \begin{tabular}{|c|}
104 \\
C104 \\
\end{tabular} & \begin{tabular}{|c|}
105 \\
C104 \\
\end{tabular} & \begin{tabular}{|c|}
171 \\
C104 \\
\end{tabular} & \begin{tabular}{|c|}
172 \\
$\mathrm{C} 104$ \\
\end{tabular} & \begin{tabular}{|c|}
109 \\
$D 101$ \\
\end{tabular} & \begin{tabular}{|c|}
112 \\
$D 101$ \\
\end{tabular} & \begin{tabular}{|c|}
108 \\
$D 101$ \\
\end{tabular} \\
\hline & Tentative Identification & RT & MW & sw & sw & ne & ne & sw & sw & ne & sw & ne & sw & e & $w$ & ne \\
\hline 128 & C3-naphthalene & 22.65 & 170 & & & & & & & & & & & & & \\
\hline 129 & phenyl maleic anhydride & 22.90 & 214 & & 0.03 & & & 0.07 & & & & & 0.14 & & & \\
\hline 130 & dibenzoturan & 22.93 & 168 & 0.01 & & & & 0.05 & 5) 0.01 & & & 0.03 & 0.10 & 0.02 & 0.05 & 0.01 \\
\hline 131 & 1 thexadecene & 22.47 & 224 & & & & & & & & & & & & & \\
\hline 132 & n-hexadecane & 23.57 & 226 & & & & & & & & & & & & 0.08 & \\
\hline 133 & benzenedicarboxylic acid, diethyi ester & 23.64 & 222 & & & & & & & & & & & & & \\
\hline 134 & 1,3-dibromo-2,2-bis(bromomethyl) propane & 24.17 & 384 & & & & & & & & & & & & & \\
\hline 135 & diphenyl diazene & 24.23 & 182 & & & & & & & & & & & & & \\
\hline 936 & benzophenone & 24.30 & 182 & & 0.01 & & & & & & & 0.03 & 0.03 & & & \\
\hline 137 & 1-heptadecene & 24.71 & 238 & & & & & & & & & & & & & \\
\hline 138 & n-heptadecane & 24.81 & 240 & & & & & & & & & & & 0.04 & 0.33 & 0.03 \\
\hline 939 & N-(phenylmethylene) benzamine & 24.83 & 181 & & & & & & & & & & & & & \\
\hline 140 & $9 \mathrm{H}$-fluoren-9-one & 25.28 & 180 & & & & & & & & & & & & 0.18 & \\
\hline 141 & tribromobenzamine & 25.39 & 327 & & & & & & & & & & & & & \\
\hline 142 & trimethyl indan & 25.45 & 160 & & & & & & & & & & & & & \\
\hline 143 & dibenzothiophene & 25.58 & 184 & & & & & & & & & & & & & \\
\hline 144 & tetrabromobenzene & 25.86 & 390 & & & & & & & & & & & & & \\
\hline 145 & In-octadecane & 25.88 & 254 & & & & & & & & & & & & 0.40 & 0.03 \\
\hline 146 & phenanthrene/anthracene & 26.37 & 178 & & & 0.03 & & 0.23 & 0.02 & 0.01 & 0.01 & & & 0.05 & 0.15 & 0.03 \\
\hline 147 & methyl dibenzothiophene & 26.98 & 198 & & & & & & & & & & & & & \\
\hline 148 & n-honadecane & 27.04 & 268 & & & & & & & & & & & & 0.14 & \\
\hline 149 & 2-methyl anthracene/phenanthrene & 27.48 & 192 & & & & & & & & & & & & & \\
\hline 150 & 1-methyl anthracene/phenanthrene & 27.57 & 192 & & & & & & & & & & & & & \\
\hline 151 & 2,6-dibutyl-2,5-cyclohexadien-1,4-dione & 31.30 & 220 & & 1.18 & & 0.29 & 0.15 & & & & 0.21 & 0.49 & & & \\
\hline 152 & Total PCB's & & & 5.50 & 0.40 & 3.80 & 1.90 & 47.10 & 3.80 & 4.50 & 1.40 & 49.80 & 21.30 & 0.20 & 0.60 & 0.20 \\
\hline & & & & & & & & & & & & & & & & \\
\hline & & & & & & & & & & & & & & & & \\
\hline & & & & & & & & & & & & & & & & \\
\hline & & & & & & & & & & & & & & & & \\
\hline & & & & & & & & & & & & & & & & \\
\hline & & & & & & & & & & & & & & & & \\
\hline & & & & & & & & & & & & & & & & \\
\hline & & & & & & & & & & & & & & & & \\
\hline & & & & & & & & & & & & & & & & \\
\hline & & & & & & & & & & & & & & & & \\
\hline & & & & & & & & & & & & & & & & \\
\hline & & & & & & & & & & & & & & & & \\
\hline & & & & & & & & & & & & & & & & \\
\hline & & & & & & & & & & & & & & • & & \\
\hline & & & & & & & & & & & & & & & & \\
\hline & & & & & & & & & & & & & & & & \\
\hline & & & & & & & & & & & & & & & & \\
\hline & & & & & & & & & & & & & & & & \\
\hline & & & & & & & & & & & & & & & & \\
\hline & & & & & & & & & & & & & & & & \\
\hline & & & & & & & & & & & & & & & & \\
\hline & & & & & & & & & & & & & & & & \\
\hline & & & & & & & & & & & & & & & & \\
\hline & & & & & & & & & & & & & & & & \\
\hline & & & & & & & & & & & & & & & & \\
\hline & & & & & & & & & & & & & & & & \\
\hline & & & & & & & & & & & & & & & & \\
\hline & & & & & & & & & & & & & & & & \\
\hline & & & & & & & & & & & & & & & & \\
\hline & & & & & & & & & & & & & & & & \\
\hline & & & & & & & & & & & & & & & & \\
\hline & & & & & & & & & & & & & & & & \\
\hline & & & & & & & & & & & & & & & & \\
\hline no & tistiont & & & & & & 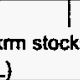 & 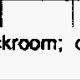 & drway & 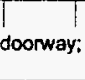 & & & & & & \\
\hline
\end{tabular}


Air Monitoring Results

Sample number and room number at the top of each data column

\begin{tabular}{|c|c|c|c|c|c|c|c|c|c|c|c|c|c|c|c|c|}
\hline & & & & 111 & 94 & 110 & 113 & 6 & 4 & 5 & 148 & 149 & 179 & 180 & 181 & 122 \\
\hline & Compound & & & D102 & D103 & D103 & 0104 & E103 & E104 & E104 & E104 & E104 & E104 & & & E104 \\
\hline & Tentative Identificalion & $\mathrm{RT}$ & MW & $m$ & $\mathrm{~m}$ & $\mathbf{s}$ & nw & $\mathrm{m} / \mathrm{r}$ & $\mathrm{m} / \mathrm{s}$ & $\mathrm{m} / \mathrm{n}$ & se & $w$ & ne & 0 & 0 & $w / m$ \\
\hline 1 & sulfur dioxide & 1.55 & 64 & & & & & & 1.49 & & & & & & & \\
\hline 2 & 2-propanone & 1.89 & 58 & & & & & & & & & & & & & \\
\hline 3 & 1-fluoro-1,1-dichloroethane & 1.95 & 116 & & & & & & & & & & & & & \\
\hline 4 & N-ethyl ethanamine & 2.36 & 73 & & & & & & & & & & & & & \\
\hline 5 & 2-butanone & 2.56 & 72 & & & & & & & & & & & & & \\
\hline 6 & N-ethyl-N-methyl ethanamine & 2.94 & 87 & & & & & & & & & & & & & \\
\hline 7 & dichloromethane & 3.15 & 84 & & & & & & & & & & & & & \\
\hline 8 & $\mathrm{~N}-(1$-methylethy) $\}$-2-propanamine & 3.59 & 101 & & & & & & & & & & & & & \\
\hline 9 & benzene & 3.64 & 78 & 0.02 & & 0.03 & 0.03 & & & 0.39 & 0.97 & 1.22 & 0.97 & 1.06 & 1.00 & \\
\hline 10 & 2-pentanone & 4.08 & 86 & & & & & & & & & & & & & \\
\hline 11 & trichloroethene & 4.47 & 130 ! & & & & & & & 0.59 & 1.12 & 1.29: & 0.35 & 0.05 & 0.04 & 0.75 \\
\hline 12 & N-(1-methylethylidene)-2-propanamine & 4.62 & 99 & & & & & & & & & & & & & \\
\hline 13 & tolvene & 6.97 & 92 & 0.11 & 0.14 & 0.13 & 0.10 & 4.84 & & 12.89 & 2.11 & 2.75 & 4.67 & 4.47 & 3.00 & 0.03 \\
\hline 14 & $\mathrm{~N}$-methyl-N-(1-methylethyl)-2-propanamine & 7.27 & 115 & & & & & & & & & & & & & \\
\hline 15 & 3-hexanone & 7.75 & 100 & & & & & & & & & & & & & \\
\hline 16 & 2-hexanone & 7.92 & 100 & & & & & & & & & & & & & \\
\hline 17 & n-octane & 8.29 & 114 & & & & & & & 0.31 & 0.43 & 0.24 & 0.18 & 0.34 & 0.22 & \\
\hline 18 & hexanal & 8.33 & 100 & & & & & & & & & & & & & \\
\hline 19 & tetrachloroethene & 8.79 & 164 & 0.00 & 0.02 & & & & & 0.31 & & 0.60 & & 0.81 & & 0.34 \\
\hline 20 & methyl ethyl disulfide & 9.68 & 108 & & & & & & & & & & & & & \\
\hline 21 & chlorobenzene & 10.05 & $1+2$ & & & & & & & & & & & & & \\
\hline 22 & ethyl benzene & 10.57 & 106 & 0.02 & 0.03 & 0.02 & 0.02 & 0.38 & & 0.87 & 0.31 & 0.49 & 0.88 & 1.03 & 0.59 & 0.01 \\
\hline 23 & p,m-xylene & 10.81 & 106 & 0.07 & 0.10 & 0.09 & 0.06 & 1.34 & & 2.86 & 0.60 & 0.92 & 2.71 & 2.56 & 1.52 & 0.04 \\
\hline 24 & 1,4-oxathiane & 11.00 & 104 & & & & & & & & & & & & & \\
\hline 25 & styrene & 11.30 & 118 & 0.01 & 0.02 & & 0.01 & & & 0.42 & & & & & & \\
\hline 26 & 3-heptanone & 11.37 & 114 & & & & & & & & 0.19 & & & & & \\
\hline 27 & o-xylene & 11.49 & 106 & 0.03 & 0.04 & 0.03 & 0.03 & 0.47 & & 1.08 & 0.48 & 0.50 & 1.61 & 1.84 & 1.11 & 0.02 \\
\hline 28 & heptanal & 11.60 & 114 & & & & & & & & & & & & & \\
\hline 29 & n-nonane & 11.67 & 128 & & & & & & & 0.21 & 0.60 & 0.30 & & & & \\
\hline 30 & C3-benzene & 12.20 & 120 & & & & & & & & & & 0.12 & 0.18 & & \\
\hline 31 & N-butylidene-1-butanamine & 12.28 & 127 & & & & & & & & & & & & & \\
\hline 32 & C3-benzene & 12.33 & 120 & & & & & & & 0.08 & & & & & & \\
\hline 33 & 3-methyl nonane & 12.51 & 142 & & & & & & & & & & & & & \\
\hline 34 & pinene & 12.61 & 136 & 0.15 & 0.26 & 0.18 & 0.04 & & & 0.45 & & & 0.25 & & & \\
\hline 35 & 2-methyl cyciohexanol & 12.62 & 114 & & & & & & & & & & & & & \\
\hline 36 & 3-octanone & 12.71 & 128 & & & & & & & & & & & & & \\
\hline 37 & 2-ethyl hexanal & 13.02 & 128 & & & & 0.03 & & & & & & & & & \\
\hline 38 & 1-chloro-2-methyl benzene & 13.03 & 126 & & & & & & & & & & & & & \\
\hline 39 & propyi benzene & 13.05 & 120 & & & & & & & 0.32 & 0.36 & 0.31 & 0.54 & 0.74 & 0.44 & \\
\hline 40 & N-butyl-1-butanamine & 13.17 & 129 & & & & & & & & & & & & & \\
\hline 41 & benzaldehyde & 13.18 & 106 & 0.03 & 0.04 & 0.02 & 0.03 & 0.30 & & 0.22 & 2.60 & 2.60 & & 3.70 & 2.40 & 0.02 \\
\hline 42 & C3-benzene & 13.25 & 120 & 0.04 & 0.06 & 0.05 & 0.03 & 0.92 & & 1.35 & & & 1.56 & 1.49 & 0.77 & \\
\hline 43 & C3-benzene & 13.41 & 120 & & 0.02 & & & 0.44 & & 0.56 & & & 0.68 & 0.70 & 0.42 & 0.01 \\
\hline 44 & phenol & 13.57 & 94 & & & & & & & & & & & & 0.37 & 0.03 \\
\hline 45 & aniline & 13.61 & 93 & & & & & & & & & & & & & \\
\hline 46 & C3-benzene & 13.68 & 120 & & & & & & & 0.63 & 0.31 & 0.42 & & & & \\
\hline 47 & 6-methyl-5-hepten-2-one & 13.69 & 126 & & & & & & & & 0.28 & & & & & \\
\hline 48 & benzonitrile & 13.72 & 103 & & & & & & & & 0.48 & 0.44 & & & & \\
\hline 49 & 1-decene & 13.83 & 140 & & & & & & & & & & & & & \\
\hline 50 & C3-benzene & 13.99 & 120 & & & & 0.05 & & & 1.56 & & & & & & \\
\hline 51 & n-decane & 14.07 & 142 & & 0.04 & & & & & 0.65 & & & & & & \\
\hline 52 & octanal & 14.09 & 128 & & & & 0.02 & & & & 0.40 & 0.17 & & & & \\
\hline 53 & dichlorobenzene & 14.40 & 146 & & & & & & & 0.25 & & & & & & 0.01 \\
\hline 54 & 4-methyl decane & 14.56 & 156 & & & & & & & & & & & & & \\
\hline 55 & C3-benzene & 14.57 & 120 & & & & & & & & 0.24 & 0.22 & 0.57 & 0.44 & 0.25 & \\
\hline 56 & C4-benzene & 14.60 & 134 & & & & & 0.38 & & 0.75 & & & & 0.55 & & \\
\hline 57 & 2-ethyl hexanol & 14.64 & 130 & & 0.11 & & 0.12 & & & & 0.37 & 0.30 & 0.94 & & & \\
\hline 58 & limonene & 14.75 & 136 & 0.03 & 0.05 & 0.03 & 0.02 & 0.21 & & 0.72 & & & 0.56 & & & 0.01 \\
\hline 59 & benzene methano: & 14.77 & 108 & & & & & & & & & & & & & \\
\hline 60 & indan & 14.87 & 118 & & & & & & & 0.24 & 0.30 & 0.21 & 0.32 & 0.29 & 0.24 & \\
\hline 61 & dichlorobenzene & 14.89 & 146 & & & & & & & & & & & 0.35 & 0.18 & \\
\hline 62 & N-ethyl-N-(1-methylethyl)-2-propanamine & 15.07 & 129 & & & & & & & & & & & & & \\
\hline 63 & C4-benzene & 15.10 & 134 & & & & & 0.24 & & 0.37 & 0.34 & 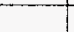 & & & & \\
\hline
\end{tabular}




\begin{tabular}{|c|c|c|c|c|c|c|c|c|c|c|c|c|c|c|c|c|}
\hline & & & & 114 & 94 & 110 & 113 & 6 & 4 & 5 & 148 & 149 & 179 & 180 & 181 & 122 \\
\hline & Compound & & & D102 & 0103 & 0103 & D104 & E103 & $E 104$ & E104 & E104 & $E 104$ & E104 & & & E104 \\
\hline & Tentative Identification & RT & MW & $\mathrm{m}$ & $m$ & s & nw & $\mathrm{m} / \mathrm{r}$ & $\mathrm{m} / \mathrm{s}$ & $\mathrm{m} / \mathrm{n}$ & se & $w$ & ne & 0 & 0 & $w / m$ \\
\hline 64 & 5-methy decane & 15.21 & 156 & & & & & & & & & & & & & \\
\hline 65 & 4-methyl decane & 15.29 & 156 & & & & & & & & & & & & & \\
\hline 66 & C4-benzene & 15.30 & 134 & & & & & 0.26 & & 0.56 & & 0.09 & 0.55 & & 0.29 & \\
\hline 67 & 2-methyl decane & 15.34 & 156 & & & & & & & & & & & & & \\
\hline 68 & 1,4-dithiane & 15.39 & 120 & & & & & & & & & & & & & \\
\hline 69 & acetophenone & 15.47 & 120 & & & & 0.03 & & & & 2.21 & 1.46 & 0.67 & 1.99 & 1.52 & 0.01 \\
\hline 70 & C4-benzene & 15.47 & 134 & & & & & & & 0.33 & 0.17 & 0.08 & 0.56 & & 0.30 & \\
\hline 71 & 3-methyl decane & 15.48 & 156 & & & & & & & & & & & & & \\
\hline 72 & methyl benzaldehyde & 15.51 & 120 & & & & & & & & & & & & & \\
\hline 73 & C4-benzene & 15.64 & 934 & & & & & & & & & & 0.20 & 0.15 & 0.09 & \\
\hline 74 & C4-benzene & 15.72 & 134 & & & & & & & 0.38 & & & 0.19 & 0.16 & & \\
\hline 75 & A,A-dimethy benzene methanoi & 15.80 & 136 & & & & & & & & & & & & & \\
\hline 76 & C4-benzene & 15.86 & 134 & & & & & 0.26 & & 0.63 & & & 0.72 & 0.69 & $0 . \overline{41}$ & \\
\hline 77 & 1-undecene & 15.95 & 154 & & & & & & & & & & & & & \\
\hline 78 & n-undecane & 16.05 & 156 & & & & & 0.40 & 0.01 & 0.40 & 0.35 & 0.42 & & & & \\
\hline 79 & nonanal & 16.12 & 142 & & 0.05 & & 0.04 & & & & 0.96 & 0.38 & 0.31 & 0.97 & 0.38 & 0.01 \\
\hline 80 & C4-benzene & 16.24 & 134 & & & & & & & & 0.14 & & 0.19 & 0.11 & & \\
\hline 81 & phosphoric acid, triethyl ester & 16.41 & 182 & & & & & & & & & & & & & \\
\hline 82 & C4-benzene & 16.43 & 134 & & & & & 0.17 & & 0.24 & & & & & & \\
\hline 83 & trans methyt decalin & 16.50 & 152 & & & & & & & & & & & & & \\
\hline 84 & C4-benzene & 16.55 & 134 & & & & & 0.21 & & 0.50 & & & & & & \\
\hline 85 & cis methyl decalin & 16.74 & 152 & & & & & & & 0.08 & & & & & & \\
\hline 86 & C5-benzene & 16.79 & 148 & & & & & & & 0.08 & 0.10 & & & & & \\
\hline 87 & methyl indan & 17.14 & 132 & & & & & 0.33 & & 0.48 & & & 0.29 & & & \\
\hline 88 & 0,0-diethyl-S ethyl phosphorothioate & 17.16 & 198 & & & & & & & & & & & & & \\
\hline 89 & methyl indan & 17.29 & 132 & & & & & & & 0.33 & 0.16 & 0.21 & & & & \\
\hline 90 & trichlorobenzene & 17.64 & 180 & & & & & & & & & & & & & \\
\hline 91 & 1-dodecene & 17.66 & 168 & & & & & & & & & & & & & \\
\hline 92 & 1-(4-methyiphenyl) ethanone & 17.67 & 134 & & & & & & & & & & & & & \\
\hline 93 & $\mathrm{~N}, \mathrm{~N}$-dibutyl-1-butanamine & 17.72 & 142 & & & & & & & & & & & & & \\
\hline 94 & n-dodecane & 17.80 & 170 & & & & & & & & 0.10 & 0.15 & 0.23 & 0.13 & 0.08 & \\
\hline 95 & naphthalene & 17.79 & 128 & 0.76 & 3.33 & 1.86 & 0.05 & 0.72 & & 0.20 & 0.41 & 0.58 & 0.90 & 0.41 & 0.29 & 0.02 \\
\hline 96 & C5-benzene & 17.87 & 948 & & & & & & & 0.18 & & & & & & \\
\hline 97 & decanal & 17.90 & 156 & & & & 0.02 & & & & 0.94 & 0.34 & 0.25 & 0.77 & 0.24 & \\
\hline 98 & benzothiophene & 17.90 & 134 & 0.03 & 0.09 & 0.06 & & & & & & & & & & \\
\hline 99 & 1,4-oxathiane, 4,4-dioxide & 18.35 & 136 & & & & & & & & & & & & & \\
\hline 100 & benzothiazole & 18.40 & 135 & & & & & & & & & & & & & \\
\hline 101 & benzene propanenitrile & 18.53 & 131 & & & & & & & & & & & & & \\
\hline 102 & dimethyl indan & 18.80 & 146 & & & & & & & 0.08 & & & & & & \\
\hline 103 & 3-tetradecene & 18.85 & 196 & & & & & & & & & & & & & \\
\hline 104 & $\mathrm{~N}$-butyl-N-nitroso $\mathbf{N}$-butanamine & 18.91 & 158 & & & & & & & & & & & & & \\
\hline 105 & 1-tridecene & 19.26 & 182 & & & & & & & & & & & & & \\
\hline 106 & n-tridecane & 19.38 & 184 & & & & & & & 0.08 & 0.13 & 0.06 & 0.11 & 0.08 & & \\
\hline 107 & $\mathrm{~N}, \mathrm{~N}$-dibutyl formamide & 19.51 & 157 & & & & & & & & & & & & & \\
\hline 108 & 2-methyl naphthalene & 19.61 & 142 & 0.18 & 1.47 & 0.43 & 0.01 & 0.32 & & 0.40 & 0.18 & 0.25 & 0.24 & 0.12 & 0.07 & 0.02 \\
\hline 109 & phthalate & 19.80 & 390 & & & & & & & & & & & & & \\
\hline 110 & 1,3-isobenzofurandione & 19.81 & 148 & & & & & & & & 0.23 & 0.11 & & 0.09 & 0.08 & \\
\hline 111 & 1-methyl naphthalene & 19.91 & 142 & 0.11 & 0.55 & 0.24 & & 0.18 & & 0.28 & 0.15 & 0.10 & 0.19 & & 0.07 & \\
\hline 112 & 1 -methy-4-(propylthio) benzene & 20.37 & 166 & & & & & & & & & & & & & \\
\hline 113 & 1-tetradecene & 20.76 & 196 & & & & & & & & 0.10 & 0.10 & & & & \\
\hline 114 & n-tetradecane & 20.86 & 198 & & & & & 0.18 & & 0.08 & & & 0.06 & 0.07 & & \\
\hline 115 & biphenyl & 20.87 & 154 & 0.05 & 0.20 & 0.08 & 0.01 & & & & & & 0.06 & & & 0.01 \\
\hline 116 & 1,1'-oxy bis(benzene) & 21.17 & 186 & & & & & & & & 0.07 & & & & & \\
\hline 117 & 2,4,6-trichloroaniline & 21.20 & 195 & & & & & & & 0.14 & 0.18 & 0.10 & 0.17 & & & \\
\hline 118 & C2-naphthaiene & 21.28 & 156 & 0.14 & 0.18 & & & & & & & & & & & 0.03 \\
\hline 119 & C2-naphthalene & 21.51 & 156 & & 0.19 & 0.05 & & & & 0.02 & 0.20 & & 0.09 & & & 0.04 \\
\hline 120 & C2-naphthalene & 21.56 & 156 & & 0.09 & & & & & & 0.20 & & & & & \\
\hline 121 & tribromobenzene & 21.62 & 312 & & & & & & & & & & & & & \\
\hline 122 & 6,10-dimethyl-5,9-undecadien-2-one & 21.65 & 194 & & & & & & & & & & & & & \\
\hline 123 & 2,6-di-t-butyt-2,5-cyclohexadiene-1,4-dione & 22.06 & 220 & & & & & & & & & & & & & \\
\hline 124 & 1-pentadecene & 22.16 & 210 & & & & & & & & & & & & & \\
\hline 125 & n-pentadecane & 22.26 & 212 & & & & & 0.09 & & & 0.08 & & 0.03 & 0.04 & & \\
\hline 126 & methyl biphenyl & 22.30 & 168 & 0.01 & & 0.02 & & & & & 0.20 & & & & & \\
\hline 127 & 1,2-dihydroacenaphthylene & 22.50 & 168 & 0.02 & 0.11 & & & & & & 0.20 & & & & & \\
\hline
\end{tabular}


Air Monitoring Results

Sample number and room number at the top of each data column

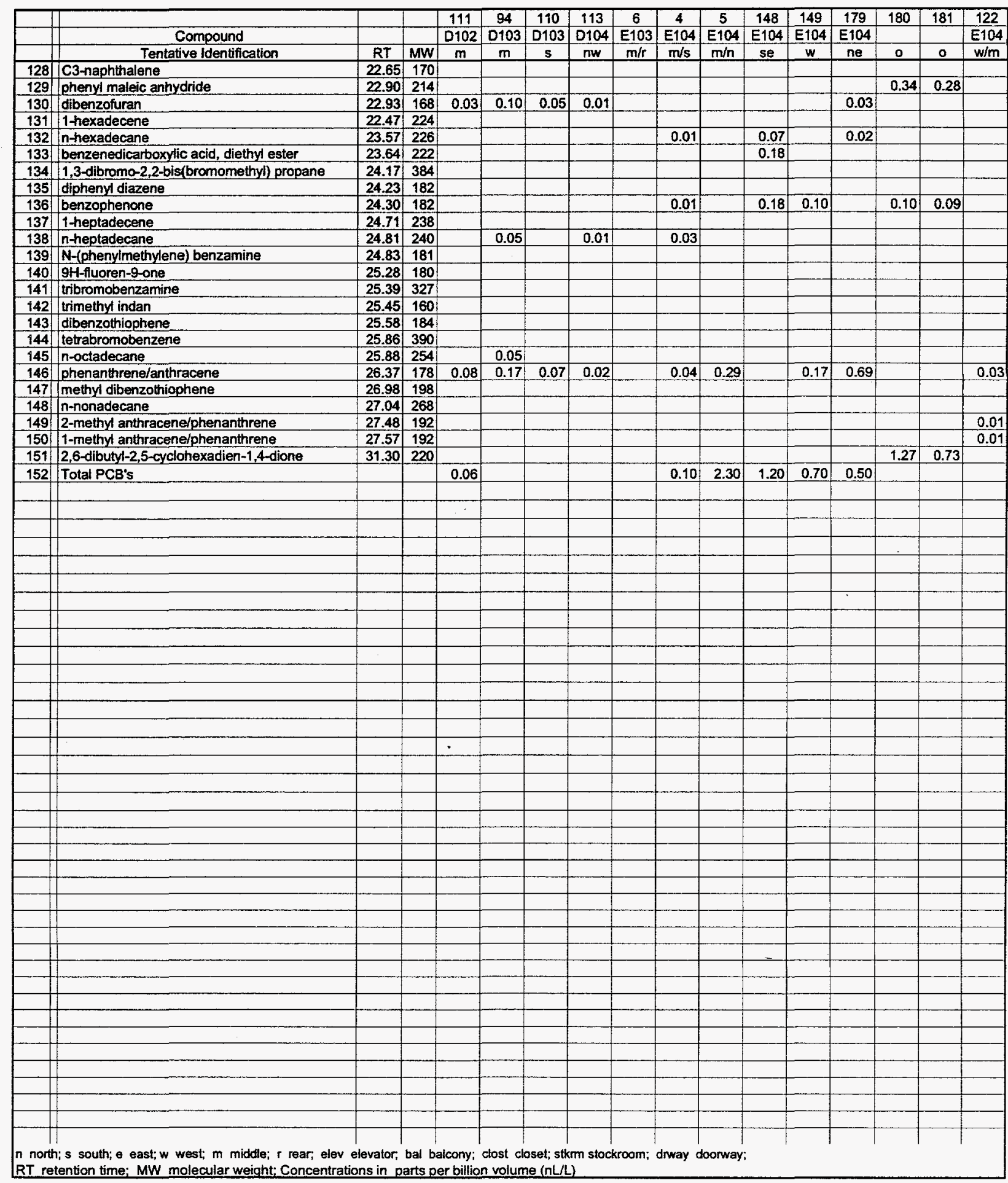




\begin{tabular}{|c|c|c|c|c|c|c|c|c|c|c|c|c|c|c|c|c|}
\hline 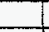 & & & & 7 & 139 & 8 & 9 & 13 & 12 & 11 & 153 & 121 & 150 & 151 & 152 & 10 \\
\hline & Compound & & & E105 & E105 & E107 & E108 & $E 113$ & E115 & E116 & E116 & E116 & E116 & & & E117 \\
\hline & Tentative Identification & RT & MW & $m$ & $\mathbf{m}$ & $\mathbf{m}$ & $\mathrm{m} / \mathrm{s}$ & $m$ & $\mathbf{m}$ & m & c & sw & sw & 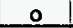 & 으 & $\mathbf{m}$ \\
\hline 1 & sulfur dioxide & 1.55 & 64 & & & & & & 1.29 & & & & & & 1.61 & \\
\hline 2 & 2-propanone & 1.89 & 58 & & & & & & & & & & & & & \\
\hline 3 & 1-fluoro-1,1-dichloroethane & 1.95 & 116 & & & 5.36 & 2.85 & & & & & & & & & \\
\hline 4. & N-ethyl ethanamine & 2.36 & 73 & & & & & & & & & 0.16 & & & & \\
\hline 5 & 2-butanone & 2.56 & 72 & & & & & & & & & 0.40 & & & & \\
\hline 6. & N-ethyl-N-methyl ethanamine & 2.94 & 87 & & & & & & & & & & & & & \\
\hline 7 & dichloromethane & 3.15 & 84 & & & & & & & & & & & & & \\
\hline 8 & $\mathrm{~N}$-(1-methylethyl)-2-propanamine & 3.59 & 101 & & & & & & & & & 19.50 & & & 0.40 & \\
\hline 9 & benzene & 3.64 & 78 & & & & & & & 0.32 & 1.76 & & 0.02 & 1.00 & 1.01 & 0.10 \\
\hline 10 & 2-pentanone & 4.08 & 86 & & & & & & & & & 0.14 & & & & \\
\hline 11 & trichloroethene & 4.47 & 130 & 0.51 & & 0.57 & & 0.13 & & 0.34 & 0.93 & 0.39 & 0.05 & 0.05 & 0.05 & \\
\hline 12 & $\mathrm{~N}$-(1-methylethylidene)-2-propanamine & 4.62 & 99 & & & & & & & & 1.34 & 0.02 & & & & \\
\hline 13 & toluene & 6.97 & 92 & 1.47 & 0.06 & 12.96 & 1.59 & 1.04 & 0.09 & 1.75 & 4.77 & 0.45 & 0.18 & 2.00 & 1.44 & 0.29 \\
\hline 14 & $\mathrm{~N}$-methyl-N-(1-methylethyl)-2-propanamine & 7.27 & 115 & & & & & & & & & 0.07 & & & & \\
\hline 45 & 3-hexanone & 7.75 & 100 & & & & & & & & & 0.09 & & & & \\
\hline 16 & 2-hexanone & 7.92 & 100 & & & & & & & & & 0.30 & & & & \\
\hline 17 & n-octane & 8.29 & 114 & & & & & & & & & & & & & \\
\hline 18 & hexanal & 8.33 & 100 & & & 0.60 & & & & & 4.10 & 0.27 & & 1.40 & & 0.08 \\
\hline 19 & tetrachloroethene & 8.79 & 164 & 0.30 & & 0.27 & & 0.14 & 0.01 & 0.23 & & 0.68 & & & & 0.10 \\
\hline 20 & Imethyl ethyl disulfide & 9.68 & 108 & & & & & & & & & 0.08 & & & & \\
\hline 21 & chlorobenzene & 10.05 & 112 & & & & & & & & & 0.23 & & & & \\
\hline 22 & ethy benzene & 10.57 & 106 & & & 0.88 & & 0.19 & 0.01 & 0.29 & 1.07 & 0.20 & 0.02 & 0.52 & 0.27 & 0.05 \\
\hline 23 & p,m-xylene & 10.81 & 106 & 0.54 & & 2.38 & 0.20 & 0.65 & 0.06 & 1.06 & 3.12 & 0.86 & 0.02 & 0.38 & 0.63 & 0.13 \\
\hline 24 & 1,4-oxathiane & 11.00 & 104 & & & & & & & & & trace & & & & \\
\hline 25 & styrene & 11.30 & 118 & & & 2.14 & 0.16 & 0.08 & & 0.36 & & & & & & \\
\hline 26 & 3-heptanone & 11.37 & 114 & & & & & & & & & 0.49 & & & & \\
\hline 27 & o-xylene & 11.49 & 106 & 0.21 & & 1.41 & & 0.30 & 0.03 & 0.43 & 1.15 & 0.27 & 0.01 & 0.17 & 0.41 & 0.12 \\
\hline 28 & heptanal & 11.60 & 114 & & & & & & & 0.24 & & & & 0.66 & & \\
\hline 29 & n-nonane & 11.67 & 128 & & & 0.90 & & 0.08 & & & 1.16 & 0.09 & & & 0.54 & \\
\hline 30 & C3-benzene & 12.20 & 120 & & & & & & & & & & & & & \\
\hline 31 & N-butylidene-1-butanamine & 12.28 & 127 & & & & & & & & & 0.07 & & & & \\
\hline 32 & C3-benzene & 12.33 & 120 & & & 0.37 & & & & & & 0.65 & & & & \\
\hline 33 & 3-methyl nonane & 12.51 & 142 & & & 0.55 & & & & & & & & & 0.24 & \\
\hline 34 & pinene & 12.61 & 136 & & & & & & & & & & & & & \\
\hline 35 & 2-methyl cyclohexanol & 12.62 & 114 & & & & & & & & & 0.67 & & & & \\
\hline 36 & 3-octanone & 12.71 & 128 & & & & & 0.15 & & & 0.58 & & & & & 0.14 \\
\hline 37 & 2-ethyl hexanal & 13.02 & 128 & & & & & & & & & & & & & \\
\hline 38 & 1-chloro-2-methyl benzene & 13.03 & 126 & & & & & & & & & 0.16 & & & & \\
\hline 39 & propyl benzene & 13.05 & 120 & & & $0 . \overline{B 2}$ & & 0.11 & & 0.27 & & & & & & 0.04 \\
\hline 40 & $\mathrm{~N}$-butyl-1-butanamine & 13.17 & 129 & & & & & & & & & 3.05 & & & & \\
\hline 41. & benzaldehyde & 13.18 & 106 & 0.21 & 0.07 & & 0.30 & 0.19 & 0.01 & 0.67 & & & 0.50 & 10.92 & 7.03 & 0.41 \\
\hline 42 & C3-benzene & 13.25 & 120 & 0.24 & & 5.06 & & 0.43 & 0.03 & 0.62 & & & & & & \\
\hline 43 & C3-benzene & 13.41 & 120 & & & 1.38 & & 0.16 & & 0.31 & & & & & & \\
\hline 44 & phenol & 13.57 & 94 & & 0.02 & & 0.34 & & & 0.51 & & & & & & 0.27 \\
\hline 45 & aniline & 13.61 & 93 & & & & & & & & & 29.14 & & & & \\
\hline 46 & C3-benzene & 13.68 & 120 & & & 1.48 & & 0.23 & 0.03 & & 0.80 & & & & & \\
\hline 47. & 6-methyl-5-hepten-2-one & 13.69 & 126 & & & & & & & & 0.48 & & & & & \\
\hline 48. & benzonitrile & 13.72 & 103 & & & & 0.38 & & & & & 0.16 & & 0.62 & 0.41 & \\
\hline 49 & 1-decene & 13.83 & 140 & & & & & & & 0.21 & & & & & & \\
\hline 50 & C3-benzene & 13.99 & 120 & & & & & & & & 1.14 & 0.13 & & & & \\
\hline 51 & n-decane & 14.07 & 142 & & & 2.68 & & 0.32 & 0.03 & 0.68 & 0.52 & 5.10 & & & 0.18 & 0.26 \\
\hline 52 & octanal & 14.09 & 128 & & & & & & & & 1.48 & & 0.24 & 1.18 & 0.31 & \\
\hline 53 & dichlorobenzene & 14.40 & 146 & & & trace & & & & 0.15 & & & & & & \\
\hline 54 & 4-methyl decane & 14.56 & 156 & & & & & & & & & 0.40 & & & 0.08 & \\
\hline 55 & C3-benzene & 14.57 & 120 & & & & & & & & 0.75 & & & & & \\
\hline 56 & C4-benzene & 14.60 & 134 & & & & & 0.24 & & & 0.93 & & & & & \\
\hline 57 & 2-ethyl hexanol & 14.64 & 130 & & & 4.30 & 0.13 & & 0.03 & 2.50 & 0.84 & 3.55 & 2.95 & 0.65 & 0.93 & 0.16 \\
\hline 58 & limonene & 14.75 & 136 & & & 1.87 & & 0.37 & 0.02 & 0.54 & 0.28 & & & & 0.38 & \\
\hline 59 & benzene methanol & 14.77 & 108 & & & & & & & & & & & & & 0.14 \\
\hline 60 & indan & 14.87 & 118 & & & & & & & & 0.59 & & & & & \\
\hline 61) & dichlorobenzene & 14.89 & 146 & & & & & & & & & & & & & \\
\hline 62 & $\mathrm{~N}$-ethyl-N-(1-methylethyl)-2-propanamine & 15.07 & 129 & & & & & & & & & & & & & \\
\hline 63 & C4-benzene & |15.10 & 134 & & & 0.79 & & 0.14 & & 0.31 & $0.51]$ & & & & & \\
\hline
\end{tabular}




\begin{tabular}{|c|c|c|c|c|c|c|c|c|c|c|c|c|c|c|c|c|}
\hline & & & & 7 & 139 & 8 & 9 & 13 & 12 & 11 & 153 & 121 & 150 & 151 & 152 & 10 \\
\hline & Compound & & & E105 & E105 & E107 & E108 & E113 & E115 & $E 116$ & E116 & E116 & E116 & & & E117 \\
\hline & Tentative Identification & RT & $\mathrm{MW}$ & $\mathrm{m}$ & $m$ & $m$ & $\mathrm{~m} / \mathrm{s}$ & $\mathrm{m}$ & $m$ & $\mathrm{~m}$ & $\mathrm{c}$ & sw & sw & 0 & 0 & $\mathrm{~m}$ \\
\hline 64 & 5-methyl decane & 15.21 & 156 & & & & & 0.06 & & & & 0.47 & & & & \\
\hline 65 & 4-methyldecane & 15.29 & 156 & & & & & & & & & 0.55 & & & & \\
\hline 66 & C4-benzene & 15.30 & 134 & & & 0.70 & & 0.12 & & 0.25 & 1.28 & & & & & \\
\hline 67 & 2 -methyl decane & 15.34 & 156 & & & & & & & & & 0.85 & & & & \\
\hline 68 & 1,4-dithiane & 15.39 & 120 & & & & & & & & & & & & & \\
\hline 69 & acetophenone & 15.47 & 120 & & 0.05 & & & 0.10 & & 0.32 & $1.36 t$ & trace & 0.17 & 7.25 & 4.18 & 0.24 \\
\hline 70 & C4-benzene & 15.47 & 134 & & & 0.77 & & 0.13 & & & & & & & & \\
\hline 79 & 3 -methyl decane & 15.48 & 156 & & & & & & & & & 1.25 & & & & \\
\hline 72 & methyl benzaldehyde & 15.51 & 120 & & & & & & & 0.27 & & & & & & \\
\hline 73 & C4-benzene & 15.64 & 134 & & & 0.31 & & & & & 0.33 & & & & & \\
\hline 74 & C4-benzene & 15.72 & 134 & & & & & & & & 1.11 & & & & & \\
\hline 75 & A,A-dimethyl benzene methanol & 15.80 & 136 & & & & & & & & & & & & & \\
\hline 76 & C4-benzene & 15.86 & 134 & & & 0.77 & & 0.13 & & 0.51 & & & & & & \\
\hline 77 & 1-undecene & 15.95 & 154 & & & & & & & & & 0.26 & & & & \\
\hline 78 & n-undecane & 16.05 & 156 & 0.21 & & 1.95 & 0.10 & 0.24 & 0.01 & 0.29 & & 6.67 & & & 0.20 & 0.09 \\
\hline 79 & nonanal & 16.12 & 142 & & 0.02 & 0.69 & & 0.31 & 0.02 & 0.49 & 3.58 & & 0.57 & 1.97 & 0.95 & 0.22 \\
\hline 80 & C4-benzene & 16.24 & 134 & & & & & & & & & & & & & \\
\hline 81 & phosphoric acid, triethyl ester & 16.41 & 182 & & & & & & & & & & & & & \\
\hline 82 & C4-benzene & 16.43 & 134 & & & 0.41 & & 0.10 & & & 0.39 & & & & & \\
\hline 83 & trans methyl decalin & 16.50 & 152 & & & & & & & & & 0.44 & & & & \\
\hline 84 & C4-benzene & 16.55 & 134 & & & 0.63 & & 0.11 & & 0.30 & 0.44 & & & & & \\
\hline 85 & cis methyl decalin & 16.74 & 152 & & & 0.39 & & & & & & & & & & \\
\hline 86 & C5-benzene & 16.79 & 148 & & & & & & & & & & & & & \\
\hline 87 & methyl indan & 17.14 & 132 & & & & & & & & & & & & & \\
\hline 88 & 0,0 -diethy-S-ethyl phosphorothioate & 17.16 & 198 & & & & & & & & & & & & & \\
\hline 89 & methyl indan & 17.29 & 132 & & & & & & & & 0.90 & & & & & \\
\hline 90 & trichlorobemzene & 17.64 & 180 & & & & & & & 0.16 & & 0.50 & & & & \\
\hline 91 & 1-dodecene & $\begin{array}{ll}17.66 \\
\end{array}$ & 168 & & & & & & & 0.17 & & & & & & \\
\hline 92 & 1-(4-methylphenyl) ethanone & 17.67 & 134 & & & & & & & & & & & & & \\
\hline 93 & $N, N$-dibutyl-1-butanamine & \begin{tabular}{l|l}
17.72 \\
\end{tabular} & 142 & & & & & & & & & 4.72 & & & & \\
\hline 94 & n-dodecane & 17.80 & 170 & & & 1.21 & & & & & 0.38 & 0.86 & & & & \\
\hline 95 & naphthalene & 17.79 & 128 & 0.25 & & & 0.19 & 0.34 & 0.02 & 0.55 & 1.29 & & & & & 0.18 \\
\hline 96 & C5-benzene & 17.87 & 148 & & & & & & & & & & & & & \\
\hline 97. & decanal & 17.90 & 156 & & & & & 0.09 & & 0.25 & 3.28 & & 0.71 & 1.49 & 1.15 & 0.13 \\
\hline 98 & benzothiophene & 17.90 & 134 & & & & & & & & & & & & & \\
\hline 99 & 1,4-oxathiane, 4,4-dioxide & 18.35 & 136 & & & & & & & & & & & & & \\
\hline 100 & benzothiazole & 18.40 & 135 & & & & & & & & & & & & & \\
\hline 101 & benzene propanenitrile & 18.53 & 131 & & & & & & & & & & & & & \\
\hline 102 & dimethyl indan & 18.80 & 146 & & & & & & & & & & & & & \\
\hline 103 & 3-tetradecene & 18.85 & 196 & & & & & & & & & & & & & \\
\hline 104 & $\mathrm{~N}$-butyl-N-nitroso $\mathrm{N}$-butanamine & 18.91 & 158 & & & & & & & & & & & & & \\
\hline 105 & 1-tridecene & 19.26 & 182 & & & & & & & 0.17 & & & & & & \\
\hline 106 & n-tridecane & 19.38 & 184 & & & 0.18 & & 0.07 & & 0.10 & 0.21 & & & & & \\
\hline 107 & $\mathrm{~N}, \mathrm{~N}$-dibutyl formamide & 19.51 & 157 & & & & & & & & 0.18 & & & & 0.13 & \\
\hline 108 & 2-methyl naphthalene & 19.61 & 142 & & & & 0.11 & 0.13 & & 0.13 & & 0.17 & & & & 0.13 \\
\hline 109 & phthalate & 19.80 & 390 & & & & & & & & & & & & & \\
\hline 110 & 1,3-sobenzofurandione & 19.81 & 148 & & & & & & & & & & 0.37 & 0.88 & 0.41 & \\
\hline 111 & 1-methyl naphthalene & 19.91 & 142 & & & & & 0.08 & & & 1.18 & & & & & 0.05 \\
\hline 112 & 1-methyl-4-(propylthio) benzene & 20.37 & 166 & & & & & 0.08 & & 0.16 & & & & & & \\
\hline 113 & 1-tetradecene & 20.76 & 196 & & & & & & & & & & & & & \\
\hline 114 & n-tetradecane & 20.86 & 198 & 0.11 & & 0.15 & 0.06 & 0.07 & & 0.16 & & & & & & \\
\hline 115 & biphenyl & 20.87 & 154 & & & & & & & & & 0.09 & & & & 0.09 \\
\hline 116 & $1,1^{\prime}-a x y$ bis(benzene) & 21.17 & 186 & 0.14 & 0.01 & & & 0.05 & & & 0.69 & & & & & 0.05 \\
\hline 117 & 2,4,6-trichioroaniline & 21.20 & 195 & & & & & & & & & & 0.04 & & & \\
\hline 118 & C2-naphthalene & 21.28 & 156 & & & & & & & & & & & & & 0.05 \\
\hline 119 & C2-naphthalene & 21.51 & 156 & & & & & & & & 0.48 & & & & & 0.09 \\
\hline 120 & C2-naphthalene & 21.56 & 156 & & & & & & & & 0.21 & & & & & \\
\hline 121 & tribromobenzene & 21.62 & 312 & & & & & & & & & & & & & \\
\hline 122 & 6,10-dimethyl-5,9-undecadien-2-one & 21.65 & 194 & & & & & & & & & & & & & \\
\hline 123 & 2,6-di-t-buty-2,5-cyclohexadiene-1,4-dione & 22.06 & 220 & & & 0.22 & & & & & & & & & & \\
\hline 124 & 1-pentadecene & 22.16 & 210 & & & & & & 0.02 & 0.13 & & & & & & \\
\hline 125 & n-pentadecane & 22.26 & 212 & & & & 0.07 & & 0.01 & & 0.13 & & 0.06 & & 0.09 & 0.02 \\
\hline 126 & methyl biphenyl & 22.30 & 168 & & & & & & & & & & & & & \\
\hline 127 & 1,2-dihydroacenaphthylene & 22.50 & 168 & & & & & & & & 0.12 & & & & & \\
\hline
\end{tabular}


Air Monitoring Results

Sample number and room number at the top of each data column

\begin{tabular}{|c|c|c|c|c|c|c|c|c|c|c|c|c|c|c|c|c|}
\hline & Compound & & & \begin{tabular}{|c|}
7 \\
E105
\end{tabular} & \begin{tabular}{|c|}
139 \\
E105 \\
\end{tabular} & \begin{tabular}{|c|}
8 \\
$E 107$ \\
\end{tabular} & \begin{tabular}{|c|}
9 \\
E108 \\
\end{tabular} & $\begin{array}{c}13 \\
E 113\end{array}$ & \begin{tabular}{|c|}
12 \\
$E 115$ \\
\end{tabular} & \begin{tabular}{|c|}
11 \\
E116
\end{tabular} & \begin{tabular}{|c|}
153 \\
E116 \\
\end{tabular} & \begin{tabular}{|c|}
121 \\
$E 116$ \\
\end{tabular} & \begin{tabular}{|c|}
150 \\
E116 \\
\end{tabular} & 151 & 152 & \begin{tabular}{|c|}
10 \\
$E 117$
\end{tabular} \\
\hline & Tentative Identification & RT & $\mathrm{MW}$ & $\mathrm{m}$ & $\mathbf{m}$ & $\mathrm{m}$ & $\mathrm{m} / \mathrm{s}$ & $m$ & $m$ & $\mathrm{~m}$ & $c$ & sw & SW & 0 & 0 & $m$ \\
\hline 128 & C3-naphthalene & 22.65 & 170 & & & & & & & & & & & & & \\
\hline 129 & phenyl maleic anhydride & 22.90 & 214 & & & & & & & & 0.21 & & & 1.26 & 0.82 & \\
\hline 130 & dibenzofuran & 22.93 & 168 & & & & & & & & 0.15 & & & & & 0.03 \\
\hline 131 & 1-hexadecene & 22.47 & 224 & & & & & & 0.01 & & & & & & & \\
\hline 432. & n-hexadecane & 23.57 & 226 & & & & & & 0.01 & & 0.10 & & 0.03 & & & 0.02 \\
\hline 133 & benzenedicarboxylic acid, diethyl ester & 23.64 & 222 & & & & & & & & & & 0.09 & & & \\
\hline 134 & 1,3-dibromo-2,2-bis(bromomethyl) propane & 24.17 & 384 & & & & & & & & & & & & & \\
\hline 135 & diphenyl diazene & 24.23 & 182 & & & & & & & & & 0.11 & & & & \\
\hline 136 & benzophenone & 24.30 & 182 & & & & & & 0.01 & & 0.24 & 0.13 & 0.04 & 0.36 & 0.32 & \\
\hline 137 & 1-heptadecene & 24.71 & 238 & & & & & & 0.01 & & & & & & & \\
\hline 138 & n-heptadecane & 24.81 & 240 & & & & & & & & & & & & & \\
\hline 139 & $\mathrm{~N}$-(phenylmethylene) benzamine & 24.83 & 181 & & & & & & & & & & & & & \\
\hline 140 & $9 \mathrm{H}$-fluoren-9-one & 25.28 & 180 & & & & & & & & & & & & & \\
\hline 141 & tribromobenzamine & 25.39 & 327 & & & & & & & & & & & & & \\
\hline 142 & trimethyl indan & 25.45 & 160 & & & & & & & & & & & & & \\
\hline 143 & dibenzothiophene & 25.58 & 184 & & & & & & & & & & & & & \\
\hline 144 & tetrabromobenzene & 25.86 & 390 & & & & & & & & & & & & & \\
\hline 145 & n-octadecane & 25.88 & 254 & & & & & & & & & & & & & \\
\hline 146 & phenanthrene/anthracene & 26.37 & 178 & & & & 0.12 & & 0.01 & 0.63 & 0.33 & & & & & 0.06 \\
\hline 147 & methyl dibenzothiophene & 26.98 & 198 & & & & & & & & & & & & & \\
\hline 148 & n-nonadecane & 27.04 & 268 & & & & & & & & & & & & & \\
\hline 149 & 2-methyl anthracene/phenanthrene & 27.48 & 192 & & & & & & & & & & & & & \\
\hline 150 & 1-methyl anthracene/phenanthrene & 27.57 & 192 & & & & & & & 0.21 & & & & & & \\
\hline 151 & 2,6-dibutyl-2,5-cyclohexadien-1,4-dione & 31.30 & 220 & & & & & & & & & & & & & \\
\hline 152 & Total PCB's & & & & & & & & 1.00 & 5.70 & 17.70 & & 0.50 & & & \\
\hline & & & & & & & & & & & & & & & & \\
\hline & & & & & & & & & & & & & & & & \\
\hline & & & & & & & & & & & & & & & & \\
\hline & & & & & & & & & & & & & & & & \\
\hline & & & & & & & & & & & & & & & & \\
\hline & & & & & & & & & & & & & & & & \\
\hline & & & & & & & & & & & & & & & & \\
\hline & & & & & & & & & & & & & & & & \\
\hline & & & & & & & & & & & & & & & & \\
\hline & & & & & & & & & & & & & & & & \\
\hline & & & & & & & & & & & & & & & & \\
\hline & & & & & & & & & & & & & & & & \\
\hline & & & & & & & & & & & & & & & & \\
\hline & & & & & & & & & & & & & & & & \\
\hline & & & & & & & & & & & & & & & & \\
\hline & & & & & & & & & & & & & & & & \\
\hline & & & & & & & & & & & & & & & & \\
\hline & & & & & & & & & & & & & & & & \\
\hline & & & & & & & & & & & & & & & & \\
\hline & & & & & & & & & & & & & & & & \\
\hline & & & & & & & & & & & & & & & & \\
\hline & & & & & & & & & & & & & & & & \\
\hline & & & & & & & & & & & & & & & & \\
\hline & & & & & & & & & & & & & & & & \\
\hline & & & & & & & & & & & & & & & & \\
\hline & $\ldots$ & & & & & & & & & & & & & & & \\
\hline & & & & & & & & & & & & & & & & \\
\hline & & & & & & & & & & & & & & & & \\
\hline & & & & & & & & & & & & & & & & \\
\hline & & & & & & & & & & & & & & & & \\
\hline & & & & & & & & & & & & & & & & \\
\hline & & & & & & & & & & & & & & & & \\
\hline & & & & & & & & & & & & & & & & \\
\hline & & & & & & & & & & & & & & & & \\
\hline & & & & & & & & & & & & & & & & \\
\hline & " & & jarts & ( & in vol & & $\mathrm{m} s \mathrm{st}$ & & vay & 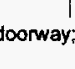 & & & & & & \\
\hline
\end{tabular}




\begin{tabular}{|c|c|c|c|c|c|c|c|c|c|c|c|c|c|c|c|}
\hline & Compound & & & \begin{tabular}{|c|}
14 \\
$E 118$ \\
\end{tabular} & $\frac{20}{E 120}$ & \begin{tabular}{|c|}
15 \\
$E 121$ \\
\end{tabular} & \begin{tabular}{|c|}
19 \\
$E 122$ \\
\end{tabular} & \begin{tabular}{|c|}
18 \\
$E 123$
\end{tabular} & \begin{tabular}{|c|}
17 \\
$E 123$ \\
\end{tabular} & \begin{tabular}{|c|}
154 \\
$E 123$ \\
\end{tabular} & \begin{tabular}{|c|}
155 \\
E123 \\
\end{tabular} & \begin{tabular}{|c|}
16 \\
$E 124$ \\
\end{tabular} & \begin{tabular}{|c|}
27 \\
E126 \\
\end{tabular} & $\begin{array}{c}28 \\
E 128\end{array}$ & $\begin{array}{c}29 \\
E 128 \\
\end{array}$ \\
\hline & Tentative Identification & RT & MW & $m$ & elev & $\mathrm{m}$ & $\mathrm{m}$ & se & $\mathrm{m} / \mathrm{n}$ & ne & $n w$ & $\mathrm{~m}$ & & $\mathrm{~m}$ & $\mathrm{~m}$ \\
\hline 1 & sulfur dioxide & 1.55 & 64 & & & & & & & & & & & & \\
\hline 2 & 2-propanone & 1.89 & 58 & & & & & & & & & & & & \\
\hline 3. & 1-fluoro-1,1-dichloroethane & 1.95 & 116 & & & & & & & & & & & & \\
\hline 4 & Nethyl ethanamine & 2.36 & 73 & & & & & & & & & & & & \\
\hline 5 & 2-butanone & 2.56 & 72 & & & & & & & & & & & & \\
\hline 6 & Nethyl-N-methyl ethanamine & 2.94 & 87 & & & & & & & & & & & & \\
\hline 7 & dichloromethane & 3.15 & 84 & & & & & & 0.18 & & & & & & \\
\hline 8 & N-(1-methylethy)-2-propanamine & 3.59 & 101 & & & & & & & & & & & & \\
\hline 9 & benzene & 3.64 & 78 & & 0.18 & & & 0.09 & 0.14 & 0.82 & 0.14 & 0.08 & & 0.52 & 0.14 \\
\hline 10 & 2-pentanone & 4.08 & 86 & & & & & & & & & & & & \\
\hline 11 & trichloroethene & 4.47 & 130 & & & & & & & 0.11 & $t$ & & & & \\
\hline 12 & $\mathrm{~N}$-(1-methylethylidene)-2-propanamine & 4.62 & 99 & & & & & & & & & & & & \\
\hline 13 & toluene & 6.97 & 92 & 0.83 & 0.71 & 0.58 & 0.54 & 0.43 & 0.52 & 1.19 & & 0.38 & 0.94 & & 0.68 \\
\hline 14 & $\mathrm{~N}$-methyl-N-(1-methylethyl)-2-propanamine & 7.27 & 115 & & & & & & & & & & & & \\
\hline 15 & 3-hexanone & 7.75 & 100 & & & & & & & & & & & & \\
\hline 16 & 2-hexanone & 7.92 & 100 & & & & & & & & & & & & \\
\hline 17) & n-octane & 8.29 & 114 & & & & & & & & & & & & \\
\hline 18 & hexanal & 8.33 & 100 & & & & & & 0.24 & 0.74 & 0.47 & & & & \\
\hline 19 & tetrachloroethene & 8.79 & 164 & 0.08 & 0.08 & 0.06 & 0.09 & & & & & & & & \\
\hline 20 & methyl ethyl disulfide & 9.68 & 108 & & & & & & & & & & & & \\
\hline 21 & chlorobenzene & 10.05 & 112 & & & & & & & & & & & & \\
\hline 22 & ethyi benzene & 10.57 & 106 & 0.12 & 0.11 & 0.09 & 0.09 & 0.08 & 0.07 & 0.25 & $t$ & 0.05 & 0.17 & 0.08 & 0.15 \\
\hline 23 & p,m-xylene & 10.81 & 106 & 0.40 & 0.31 & 0.22 & 0.24 & 0.22 & 0.18 & 0.49 & $t$ & 0.13 & 0.91 & 0.30 & \\
\hline 24 & 1,4-oxathiane & 11.00 & 104 & & & & & & & & & & & & 0.55 \\
\hline 25 & styrene & 11.30 & 118 & & & & & 0.06 & & & & & & & \\
\hline 26 & 3-heptanone & 19.37 & 114 & & & & & & & & & & & & \\
\hline 27 & Oxylene & 11.49 & 106 & & & 0.16 & 0.10 & 0.11 & 0.15 & 0.34 & & 0.12 & & & 0.20 \\
\hline 28 & heptanal & 11.60 & 114 & & & & & & & & & & & & \\
\hline 29 & n-nonane & 11.67 & 128 & 0.07 & & 0.07 & & 0.04 & 0.06 & 0.5 & & 0.04 & & & \\
\hline 30 & C3-benzene & 1220 & 120 & & & & & & & & & & & & \\
\hline 31 & N-butylidene-1-butanamine & 12.28 & 127 & & & & & & & & & & & & \\
\hline 32 & C3-benzene & 12.33 & 120 & & & & & & & & & & & & \\
\hline 33 & 3-methyl nonane & 12.51 & 142 & & & & & & & & & & & & \\
\hline 34 & pinene & 12.61 & 136 & & & & & 0.04 & & & & & & & \\
\hline 35 & 2-methyl cyclohexanol & 12.62 & 114 & & & & & & & & & & & & \\
\hline 36 & 3-octanone & 12.71 & 128 & & & 0.07 & & & & & & & 0.26 & & \\
\hline 37 & 2-ethyl hexanal & 13.02 & 128. & & & & 0.14 & & 0.03 & & & & & & \\
\hline 38 & 1-chloro-2-methyl benzene & 13.03 & 126 & & & & & & & & & & & & \\
\hline 39 & propyl benzene & 13.05 & 120 & 0.09 & & & & 0.05 & 0.04 & & & & 0.14 & & 0.09 \\
\hline 40 & N-butyl-1-butanamine & 13.17 & 129 & & & & & & & & & & & & \\
\hline 41 & benzaldehyde & 13.18 & 106 & 0.21 & 0.16 & 0.41 & 0.11 & 0.17 & 0.14 & 3.2 & 0.5 & 0.12 & & 0.06 & 0.09 \\
\hline 42 & C3-benzene & 13.25 & 120 & 0.32 & 0.20 & & 0.16 & 0.16 & 0.14 & & & 0.12 & 0.52 & 0.19 & 0.26 \\
\hline 43 & C3-benzene & 13.41 & 120 & & & & & & & & & & 0.33 & 0.06 & 0.10 \\
\hline 44 & phenol & 13.57 & 94 & 0.44 & 0.10 & 0.11 & 0.14 & 0.25 & 0.12 & & 0.47 & 0.24 & & & \\
\hline 45 & aniline & 13.61 & 93 & & & & & & & & & & & & \\
\hline 46 & C3-benzene & 13.68 & 120 & & 0.10 & & & & & & & & 0.42 & 0.07 & 0.10 \\
\hline 47 & 6-methyl-5-hepten-2-one & 13.69 & 126 & & & 0.28 & 0.22 & & 0.16 & & & & & & \\
\hline 48 & benzonitrile & 13.72 & 103 & & 0.10 & & & & & 0.33 & & & & & \\
\hline 49 & 1-decene & 13.83 & 140 & & & & & & & & & & & & \\
\hline 50 & C3-benzene & 13.99 & 120 & & & & & & & 0.22 & & & 1.43 & 0.43 & 0.81 \\
\hline 51 & n-decane & 14.07 & 142 & 0.42 & & 0.29 & & & 0.18 & & & 0.16 & 0.29 & 0.07 & 0.12 \\
\hline 52 & octanal & 14.09 & 128 & & & & 0.13 & & & 0.72 & 0.43 & & & & \\
\hline 53 & dichlorobenzene & 14.40 & 146 & & & & & & & & & & & & \\
\hline 54 & 4-methyl decane & 14.56 & 156 & & & & & & & & & & 0.09 & & \\
\hline 55 & C3-benzene & 14.57 & 120 & & 0.14 & & & & & & & & 0.55 & & \\
\hline 56 & C4-benzene & 14.60 & 134 & & 0.14 & & & & & & & & & & \\
\hline 57 & 2-ethyl hexanal & 14.64 & 130 & 0.31 & & 0.80 & 2.18 & 0.12 & 0.99 & 3.39 & 1.00 & 0.27 & & 0.33 & 0.50 \\
\hline 58 & limonene & 14.75 & 136 & 0.15 & 0.08 & 0.35 & & 0.11 & & & & 0.16 & 0.11 & 0.12 & 0.12 \\
\hline 59 & benzene methanol & 14.77 & 108 & & & & & & & & & & & & \\
\hline 60 & indan & 14.87 & 118 & & & & & & & & & & & & \\
\hline 61 & dichlorobenzene & 14.89 & 146 & & & & & & & & & & & & \\
\hline 62 & $\mathrm{~N}$-ethyl-N-(1-methylethyl)-2-propanamine & 15.07 & 129 & & & & & & & & & & & & \\
\hline 63 & C4-benzene & 15.10 & 134 & 0.11 & & 0.08 & & 0.05 & & & & & 0.23 & 0.08 & 0.10 \\
\hline
\end{tabular}




\begin{tabular}{|c|c|c|c|c|c|c|c|c|c|c|c|c|c|c|c|}
\hline & & & & 14 & 20 & 15 & 19 & 18 & 17 & 154 & 155 & 16 & 27 & 28 & 29 \\
\hline & Compound & & & E118 & E120 & E121 & E122 & E123 & E123 & E123 & E123 & E124 & E126 & E128 & E128 \\
\hline & Tentative Identification & RT & MW & $\mathrm{m}$ & elev & $m$ & $m$ & se & $\mathrm{m} / \mathrm{n}$ & ne & nw & $m$ & & $\mathbf{m}$ & $m$ \\
\hline 64 & 5-methyl decane & 15.21 & 156 & & & & & & & & & & 0.08 & & \\
\hline 65 & 4-methyl decane & 15.29 & 156 & & & & & & & & & & & & \\
\hline 66 & C4-benzene & 15.30 & 134 & 0.10 & & & & 0.07 & & & & & 0.23 & 0.13 & 0.21 \\
\hline 67 & 2-methyl decane & 15.34 & 156 & & & & & & & & & & & & \\
\hline 68 & 1,4-dithiane & 15.39 & 120 & & & & & & & & & & & & \\
\hline 69 & acetophenone & 15.47 & 120 & 0.10 & 0.16 & 0.20 & & 0.10 & 0.16 & 2.00 & 0.40 & 0.15 & & & \\
\hline 70 & C4-benzene & 15.47 & 134 & 0.12 & & & & & & 0.24 & & & & & 0.08 \\
\hline 71 & 3-methyl decane & 15.48 & 156 & & & & & & & & & & 0.17 & & \\
\hline 72 & methyl benzaldehyde & 15.51 & 120 & & & & & & & & & & & & \\
\hline 73 & C4-benzene & 15.64 & 134 & & & & & & & & & & & & \\
\hline 74 & C4-benzene & 15.72 & 134 & & & & & & & & & & 0.23 & 0.04 & 0.11 \\
\hline 75 & A,A-dimethyl benzene methanol & 15.80 & 136 & 0.08 & & 0.08 & & & & & & & & & \\
\hline 76 & C4-benzene & 15.86 & 134 & 0.08 & & & & 0.06 & & & & & 0.21 & 0.07 & 0.10 \\
\hline 77 & 1-undecene & 15.95 & 154 & & & & & & & & & & & & \\
\hline 78 & n-undecane & 16.05 & 156 & 0.22 & & 0.13 & & 0.03 & 0.07 & & & 0.06 & 0.24 & 0.09 & 0.12 \\
\hline 79 & nonanal & 16.12 & 142 & 0.21 & 0.12 & 0.25 & 0.14 & 0.11 & 0.29 & 2.03 & 1.04 & 0.23 & & 0.06 & 0.15 \\
\hline 80 & C4-benzene & 16.24 & 134 & & & & & & & & & & & & \\
\hline 81 & phosphoric acid, triethyl ester & 16.41 & 182 & & & & & & & & & & & & \\
\hline 82 & C4-benzene & 16.43 & 134 & & & & & & & & & & 0.15 & 0.06 & 0.08 \\
\hline 83 & trans methyl decalin & 16.50 & 152 & & & & & & & & & & & & \\
\hline 84 & C4-benzene & 16.55 & 134 & & & & & & & & & & 0.15 & 0.06 & 0.08 \\
\hline 85 & cis methyl decalin & 16.74 & 152 & & & & & & & & & & & & \\
\hline 86 & C5-benzene & 16.79 & 148 & & & & & & & & & & & & \\
\hline 87 & methyl indan & 17.14 & 132 & & & & & & & & & & 0.27 & 0.06 & 0.11 \\
\hline 88 & 0,0-diethy-S-ethyl phosphorothioate & 17.16 & 198 & & & & & & & & & & & & \\
\hline 89 & methyl indan & 17.29 & 132 & & & & & & & & & & & & \\
\hline 90. & trichlorobenzene & 17.64 & 180 & & & & & & & & & & & & \\
\hline 91. & 1-dodecene & 17.66 & 168 & & & & & & & & & & & & \\
\hline 92 & 1-(4-methylphenyl) ethanone & 17.67 & 134 & & & 0.05 & & & & & & & & & \\
\hline 93 & N,N-dibutyl-1-butanamine & 17.72 & 142 & & & & & & & & & & & & \\
\hline 94 & n-dodecane & 17.80 & 170 & & & & & & & & & & & & \\
\hline 95 & naphthalene & 17.79 & 128 & 0.28 & 0.25 & 0.42 & 0.22 & 0.18 & 0.16 & 0.62 & & 0.14 & & 0.20 & 0.24 \\
\hline 96. & C5-benzene & 17.87 & 148 & & & & & & & & & & & & \\
\hline 97 & decanal & 17.90 & 156 & 0.06 & 0.08 & 0.17 & 0.13 & 0.06 & 0.11 & 2.03 & 1.41 & 0.08 & & 0.07 & 0.12 \\
\hline 98 & benzothiophene & 17.90 & 134 & & & & & & & & & & & & \\
\hline 99 & 1,4-oxathiane, 4,4-dioxide & 18.35 & 136 & & & & & & & & & & & & \\
\hline 100 & benzothiazole & 18.40 & 135 & & & & & & & & 0.08 & & & & \\
\hline 101 & benzene propanenitrile & 18.53 & 131 & & & & & & & & & & & & \\
\hline 102 & dimethyl indan & 18.80 & 146 & & & & & & & & & & & & \\
\hline 103 & 3-tetradecene & 18.85 & 196 & & & & & & & & & & & & \\
\hline 104 & $\mathrm{~N}$-butyl-N-nitroso N-butanamine & 18.91 & 158 & & & & & & & & & & & & \\
\hline 105 & 1-tridecene & 19.26 & 182 & & & & & & & & & & & & \\
\hline 106 & n-tridecane & 19.38 & 184 & 0.07 & & & & & & & & & & 0.05 & 0.06 \\
\hline 107 & $\mathrm{~N}, \mathrm{~N}$-dibutyl formamide & 19.51 & 157 & & & & & & & & & & & & \\
\hline 108 & 2-methyl naphthalene & 19.61 & 142 & 0.17 & 0.06 & 0.16 & & 0.04 & 0.07 & 0.48 & & 0.06 & 0.06 & 0.07 & 0.14 \\
\hline 109 & phthalate & 19.80 & 390 & & & & & & & & & & & & \\
\hline 110 & 1,3-isobenzofurandione & 19.81 & 148 & & & & 0.03 & & & 1.56 & 0.72 & & & & \\
\hline 111 & 1-methyl naphthalene & 19.91 & 142 & 0.09 & & 0.11 & & & & & & & & 0.08 & 0.10 \\
\hline 112 & 1-methyl-4-(propylthio) benzene & 20.37 & 166 & & & & & & & & & & & & \\
\hline 113 & 1-tetradecene & 20.76 & 196 & & & & & & & & & & & & \\
\hline 114 & n-tetradecane & 20.86 & 198 & 0.07 & & & & & & & & & & 0.06 & 0.08 \\
\hline 115 & biphenyl & 20.87 & 154 & & & 0.07 & & 0.03 & 0.03 & & & 0.03 & & & \\
\hline 116 & 1,1'-oxy bis(benzene) & 21.17 & 186) & & & 0.04 & & & & & & & & & \\
\hline 117 & 2,4,6-trichloroaniline & 21.20 & 195 & & & & & & & & 0.13 & & & & \\
\hline 118 & C2-naphthalene & 21.28 & 156 & 0.13 & & 0.08 & & & & & & & & & \\
\hline 119 & C2-naphthaiene & 21.51 & 156 & 0.10 & & 0.05 & & & & & & & & 0.03 & 0.07 \\
\hline 120 & C2-naphthalene & 21.56 & 156 & 0.08 & & 0.03 & & & & & & & & & \\
\hline 121 & tribromobenzene & 21.62 & 312 & & & & & & & & & & & & \\
\hline 122 & 6,10-dimethyl-5,9-undecadien-2-one & 21.65 & 194 & & & & 0.04 & & & & & & & & \\
\hline 123 & 2,6-di-t-butyl-2,5-cyclohexadiene-1,4-dione & 22.06 & 220 & & & & & & & & & 0.03 & & & \\
\hline 124 & 1-pentadecene & 22.16 & 210 & & & & & & & & & & & & \\
\hline 125 & n-pentadecane & 22.26 & 212 & & & & & & & & & & & 0.02 & \\
\hline 126 & methyl biphenyl & 22.30 & 168 & & & & & & & & & & & & \\
\hline 127 & 1,2-dihydroacenaphthylene & 22.50 & 168 & & & & & & & & & & & & \\
\hline
\end{tabular}


Air Monitoring Results

Sample number and room number at the top of each data column

\begin{tabular}{|c|c|c|c|c|c|c|c|c|c|c|c|c|c|c|c|}
\hline & & & & 14 & 20 & 15 & 19 & 18 & 17 & 154 & 155 & 16 & 27 & 28 & 29 \\
\hline & Compound & & & E†18 & $E 120$ & $E 121$ & E122 & E123 & $E 123$ & E123 & E123 & E124 & E126 & $E 128$ & E128 \\
\hline & Tentative Identification & RT & MW & $\mathrm{m}$ & elev & m & $\mathrm{m}$ & se & $\mathrm{m} / \mathrm{n}$ & ne & nw & m & & m & m \\
\hline 128 & C3-naphthalene & 22.65 & 170 & 0.03 & & & & & & & & & & & \\
\hline 129 & phenyl maleic anhydride & 22.90 & 214 & & & & & & & & & & & & \\
\hline 130 & dibenzofuran & 22.93 & 168 & & & 0.01 & & & & 0.11 & & & & & \\
\hline 131. & 1-hexadecene & 22.47 & 224 & & & & & & & & & & & & \\
\hline 132 & n-hexadecane & 23.57 & 226 & & & & & & & & & & & & \\
\hline 133 & benzenedicarboxylic acid, diethyl ester & 23.64 & 222 & & & & & & & & & 0.03 & & & \\
\hline 134 & 1,3-dibromo-2,2-bis(bromomethyl) propane & 24.17 & 384 & & & & & & & & & & & & \\
\hline 135 & diphenyl diazene & 24.23 & 182 & & & & & & & & & & & & \\
\hline 136 & benzophenone & 24.30 & 182 & & & & & & & 0.18 & & & & & \\
\hline 137 & 1-heptadecene & 24.71 & 238 & & & & & & & & & & & & \\
\hline 138 & n-heptadecane & 24.81 & 240 & & & & & & & & & & & & \\
\hline 139 & $\mathrm{N}$-(phenylmethylene) benzamine & 24.83 & 181 & & & & & & & & & & & & \\
\hline 140 & 9H-fluoren-9-one & 25.28 & 180 & & & & & & 0.32 & & & & & & \\
\hline 141 & tribromobenzamine & 25.39 & 327 & & & & & & & & & & & & \\
\hline 142 & trimethyl indan & 25.45 & 160 & & & & & & & & & & & & \\
\hline 143 & dibenzothiophene & 25.58 & 184 & & & & & & 0.29 & & & & & & \\
\hline 144 & tetrabromobenzene & 25.86 & 390 & & & & & & & & & & & & \\
\hline 145 & n-octadecane & 25.88 & 254 & & & & & & 0.13 & & & & & & \\
\hline 146 & phenanthrene/anthracene & 26.37 & 178 & 0.06 & & 0.07 & & & 1.23 & 0.37 & & 0.10 & & & \\
\hline 147 & methyl dibenzothiophene & 26.98 & 198 & & & & & & 0.19 & & & & & & \\
\hline 148 & n-nonadecane & 27.04 & 268 & & & & & & 0.12 & & & & & & \\
\hline 149: & 2-methyl anthracene/phenanthrene & 27.48 & 192 & & & & & & 0.26 & & & & & & \\
\hline 150 & 1-methyl anthracene/phenanthrene & 27.57 & 192 & & & & & & 0.75 & & & & & & \\
\hline 151 & 2,6-dibutyl-2,5-cyclohexadien-1,4-dione & 31.30 & 220 & & & & & & & & & & & & \\
\hline 152 & Total PCB's & & & & & & & & 9.80 & 10. & 0.9 & & & & \\
\hline & & & & & & & & & & & & & & & \\
\hline & & & & & & & & & & & & & & & \\
\hline & & & & & & & & & & & & & & & \\
\hline & & & & & & & & & & & & & & & \\
\hline & & & & & & & & & & & & & & & \\
\hline & & & & & & & & & & & & & & & \\
\hline & & & & & & & & & & & & & & & \\
\hline & & & & & & & & & & & & & & & \\
\hline & & & & & & & & & & & & & & & \\
\hline & & & & & & & & & & & & & & & \\
\hline & & & & & & & & & & & & & & & \\
\hline & & & & & & & & & & & & & & & \\
\hline & & & & & & & & & & & & & & & \\
\hline & & & & & & & & & & & & & & & \\
\hline & & & & & & & & & & & & & & & \\
\hline & & & & & & & & & & & & & & & \\
\hline & & & & & & & & & & & & & & & \\
\hline & & & & & & & & & & & & & & & \\
\hline & & & & & & & & & & & & & & & \\
\hline & & & & & & & & & & & & & & & \\
\hline & & & & & & & & & & & & & & & \\
\hline & & & & & & & & & & & & & & & \\
\hline & & & & & & & & & & & & & & & \\
\hline & & & & & & & & & & & & & & & \\
\hline & & & & & & & & & & & & & & & \\
\hline & & & & & & & & & & & & & & & \\
\hline & & & & & & & & - & & . & & 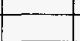 & & 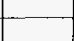 & \\
\hline & & & & & & & & & & & & & & & \\
\hline & & & & & & & & & & & & & & & \\
\hline & & & & & & & & & & & & & & & \\
\hline & & & & & & & & & & & & & & & \\
\hline & & & & & & & & & & 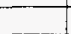 & & & & & \\
\hline & 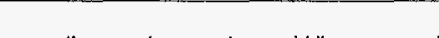 & . & & & & & & & 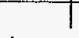 & & & & & & \\
\hline
\end{tabular}




\begin{tabular}{|c|c|c|c|c|c|c|c|c|c|c|c|c|c|c|c|c|}
\hline & & & & 25 & 24 & 23 & 26 & 124 & 123 & 141 & 142 & 146 & 147 & 178 & 21 & 127 \\
\hline & Compound & & & E129 & $E 129$ & E131 & $E 131$ & E131 & E131 & E131 & E131 & E131 & $E 131$ & E131 & $E 132$ & $E 133$ \\
\hline & Tentative Identification & RT & MW & $\mathrm{m} / \mathrm{e}$ & ne & $n$ & s & s & $n$ & s & $n w$ & $n w$ & ne & sump & $m$ & $m$ \\
\hline 1 & sulfur dioxide & 1.55 & 64 & & & & & & & & & & & & & \\
\hline 2 & 2-propanone & 1.89 & 58 & & & & & & & & & & & & & \\
\hline 3 & 1-fluoro-1,1-dichloroethane & 1.95 & 116 & & & & & & & & & & & & & \\
\hline 4 & N-ethyl ethanamine & 2.36 & 73 & & & & & & & & & & & & & \\
\hline 5 & 2-butanone & 2.56 & 72 & & & & & & & & & & & & & \\
\hline 6 & N-ethyl-N-methyl ethanamine & 2.94 & 87 & & & & & & & & & & & & & \\
\hline 7 & dichloromethane & 3.15 & 84 & & & & & & & & 0.32 & & & & & 0.36 \\
\hline 8 & $\mathrm{~N}$-(1-methylethy)-2-propanamine & 3.59 & 101 & & & & & 0.24 & & 0.21 & & 0.74 & & 0.23 & & 0.20 \\
\hline 9 & benzene & 3.64 & 78 & & & & & & 0.06 & & & 0.88 & 1.05 & 1.36 & & \\
\hline 10 & 2-pentanone & 4.08 & 86 & & & & & & & & & & & & & \\
\hline 11 & trichloroethene & 4.47 & 130 & & & 0.36 & 0.14 & & 0.24 & 0.03 & 0.04 & 1.29 & 1.06 & 0.57 & 0.33 & \\
\hline 12 & $\mathrm{~N}$-(1-methyiethylidene)-2-propanamine & 4.62 & 99 & & & & & & & & & & & & & \\
\hline 13 & toluene & 6.97 & 92 & 0.64 & 0.75 & 1.29 & 0.48 & 0.13 & 0.03 & 0.12 & 0.05 & 2.57 & 2.42 & 4.79 & 1.04 & \\
\hline 14 & N-methyl-N-(1-methylethyl)-2-propanamine & 7.27 & 115 & & & & & & & & & & & & & \\
\hline 15 & 3-hexanone & 7.75 & 100 & & & & & & & & & & & & & \\
\hline 16 & 2-hexanone & 7.92 & 100 & & & & & & & & & & & & & \\
\hline 17 & n-octane & 8.29 & 114 & & & & & & & & & & & 0.15 & & \\
\hline 18 & Ihexanal & 8.33 & 100 & & & & & & & & & 0.47 & 0.30 & & & \\
\hline 19 & tetrachloroethene & 8.79 & 164 & 0.17 & 0.16 & 0.52 & 0.18 & 0.02 & 0.15 & 0.04 & 0.04 & 1.43 & 0.60 & & 0.52 & \\
\hline 20 & methyl ethyl disulfide & 9.68 & 108 & & & & & & & & & & & & & \\
\hline 21 & chlorobenzene & 10.05 & 112 & & & & & & & & & & & & & \\
\hline 22 & ethyl benzene & 10.57 & 106 & 0.15 & 0.15 & 0.32 & 0.12 & 0.03 & 0.03 & & & 0.48 & 0.41 & 0.91 & 0.33 & \\
\hline 23 & p,m-xylene & 10.81 & 106 & 0.92 & 1.10 & 1.98 & 0.86 & 0.20 & 0.22 & 0.21 & 0.06 & 3.12 & 0.82 & 3.55 & 2.61 & 0.04 \\
\hline 24 & 1,4-oxathiane & 11.00 & 104 & & & 0.32 & & 0.01 & & & & 0.18 & 0.02 & 0.01 & 0.33 & 0.02 \\
\hline 25 & styrene & 11.30 & 118 & & & 0.18 & & 0.01 & & & & & & & 0.17 & \\
\hline 26 & 3-heptanone & 11.37 & 114 & & & & & & & & & & & & & \\
\hline 27 & o-xyiene & 11.49 & 106 & 0.53 & 0.58 & 0.98 & 0.49 & 0.10 & 0.09 & $0 . \overline{10}$ & 0.03 & 1.73 & 0.49 & 2.20 & 1.25 & 0.02 \\
\hline 28 & heptanal & 11.60 & 114 & & & & & & & & & & & & & \\
\hline 29 & n-nonane & 11.67 & 128 & & & 0.14 & & 0.02 & & & & 0.40 & 0.30 & & 0.16 & \\
\hline 30 & C3-benzene & 12.20 & 120 & & & & & 0.01 & 0.01 & & & & & 0.26 & & \\
\hline 31 & N-butylidene-1-butanamine & 12.28 & 127 & & & & & & & & & & & & & \\
\hline 32 & C3-benzene & 12.33 & 120 & & & & & & & & & 0.28 & & & & \\
\hline 33 & 3-methyl nonane & 12.51 & 142 & & & & & & & & & & & & & \\
\hline 34 & pinene & 12.61 & 136 & & & 0.22 & & 0.02 & & & & 0.12 & & 0.46 & & \\
\hline 35 & 2-methyl cyclohexanol & 12.62 & 114 & & & & & & & & & & & & & \\
\hline 36 & 3-octanone & 12.71 & 128 & & & 0.17 & & 0.04 & & & & 0.46 & & & 0.26 & \\
\hline 37 & 2-ethyl hexanal & 13.02 & 128 & & & & & & & & & & & & & \\
\hline 38 & 1-chloro-2-methyl benzene & 13.03 & 126 & & & & & & & & & & & & & \\
\hline 39 & propyl benzene & 13.05 & 120 & 0.10 & 0.11 & 0.27 & 0.11 & 0.02 & & & & 0.52 & 0.30 & 0.64 & 0.26 & \\
\hline 40 & N-butyl-1-butanamine & 13.17 & 129 & & & & & & & & & & & & & \\
\hline 41 & benzaldehyde & 13.18 & 106 & 0.16 & 0.13 & 0.31 & & 0.03 & 0.02 & $0 . \overline{12}$ & & 2.30 & 1.90 & & 0.31 & \\
\hline 42 & C3-benzene & 13.25 & 120 & 0.40 & 0.43 & 0.66 & 0.62 & 0.08 & & 0.04 & & & & 1.75 & 0.74 & \\
\hline 43 & C3-benzene & 13.41 & 120 & 0.26 & 0.29 & 0.52 & 0.27 & 0.04 & & 0.05 & & 0.68 & 0.38 & 0.76 & 0.66 & 0.01 \\
\hline 44 & phenol & 13.57 & 94 & & & & 0.24 & 0.03 & 0.02 & 0.04 & & & & & & \\
\hline 45 & aniline & 13.61 & 93 & & & & & & & & & & & & & \\
\hline 46 & C3-benzene & 13.68 & 120 & 0.16 & 0.19 & 0.34 & 0.13 & 0.05 & & 0.12 & & 1.51 & 0.35 & & 0.32 & \\
\hline 47 & 6-methyl-5-hepten-2-one & 13.69 & 126 & & & & & & & & & 0.21 & 0.24 & & 0.27 & \\
\hline 48 & benzonitrile & 13.72 & 103 & & & & & & 0.01 & & & & & & & \\
\hline 49 & 1-decene & 13.83 & 140 & & & & & & & & & & & & & \\
\hline 50 & C3-benzene & 13.99 & 120 & 0.91 & 1.12 & 1.68 & 0.99 & 0.18 & & & 0.02 & & 0.27 & & 1.91 & 0.03 \\
\hline 51 & n-decane & 14.07 & 142 & 0.29 & 0.35 & 0.57 & 0.30 & 0.05 & & 0.06 & & & & & 0.73 & 0.01 \\
\hline 52 & Toctanal & 14.09 & 128 & & & & & & & & & 0.29 & 0.25 & & & \\
\hline 53 & dichlorabenzene & 14.40 & 146 & & & trace & & 0.03 & 0.06 & & & & & & & \\
\hline 54 & 4-methyl decane & 14.56 & 156 & 0.04 & 0.12 & 0.20 & 0.08 & & & & & 0.28 & & & 0.29 & \\
\hline 55 & C3-benzene & 14.57 & 120 & & & & & & & 0.06 & & 0.93 & 0.35 & 0.75 & & 0.01 \\
\hline 56 & C4-benzene & 14.60 & 134 & & & & & & & & & 0.23 & 0.41 & & & \\
\hline 57 & 2-ethyl hexanol & 14.64 & 130 & 0.54 & 0.80 & 2.09 & 0.97 & 0.10 & 0.02 & 0.13 & 0.03 & 1.51 & 0.46 & 0.72 & 1.93 & \\
\hline 58 & limonene & 14.75 & 136 & & & & & 0.01 & & & & & & 0.64 & 0.22 & \\
\hline 59 & benzene methanol & 14.77 & 108 & & & 0.25 & & & & & & & & & & \\
\hline 60 & indan & 14.87 & 118 & & & & & & & & & 0.53 & 0,20 & 0.32 & & \\
\hline 61 & dichlorobenzene & 14.89 & 146 & & 0.23 & 0.19 & & & 0.05 & & & & & & 0.50 & \\
\hline 62 & $\mathrm{~N}$-ethyi- $\mathrm{N}$-(1-methylethy)-2-propanamine & 15.07 & 129 & & & & & & & & & 0.33 & & & 0.78 & \\
\hline 63 & C4-benzene & 15.10 & 134 & 0.20 & 0.26 & $|0.41|$ & 0.19 & & & 0.03 & & & & & 0.52 & \\
\hline
\end{tabular}




\section{Air Monitoring Results}

Sample number and room number at the top of each data column

\begin{tabular}{|c|c|c|c|c|c|c|c|c|c|c|c|c|c|c|c|c|}
\hline & & & & 25 & 24 & 23 & 26 & 124 & 123 & 141 & 142 & 146 & 147 & 178 & 21 & 127 \\
\hline & Compound & & & E129 & E129 & E131 & E131 & E131 & E131 & E131 & E131: & E131 & E131 & E131 & E132 & E133 \\
\hline & Tentative Identification & RT & MW & $\mathrm{m} / \mathrm{e}$ & ne & $n$ & $\mathbf{s}$ & $\mathbf{s}$ & $n$ & $s$ & nw & $n w$ & ne & sump & $\mathrm{m}$ & $m$ \\
\hline 64 & 5-methyl decane & 15.21 & 156 & 0.07 & & 0.14 & & & & & & & & & 0.19 & \\
\hline 65 & 4-methyl decane & 15.29 & 156 & & & & & & & & & & & & & \\
\hline 66 & C4-benzene & 15.30 & 134 & 0.16 & 0.19 & 0.30 & 0.13 & & & 0.02 & & & 0.12 & 0.58 & 0.39 & \\
\hline 67 & 2-methyi decane & 15.34 & 156 & & & 0.15 & & & & & & & & & 0.23 & \\
\hline 68 & 1,4-dithiane & 15.39 & 120 & & & & & & & & & & & & & \\
\hline 69 & acetophenone & 15.47 & 120 & & & & & 0.01 & 0.01 & 0.10 & 0.02 & 1.19 & 0.95 & 0.73 & & \\
\hline 70 & C4-benzene & 15.47 & 134 & & 0.23 & & 0.19 & & & & & 0.39 & 0.08 & 0.49 & & \\
\hline 71 & 3-methyl decane & 15.48 & 156 & 0.16 & & 0.37 & & & & & & & & & 0.46 & \\
\hline 72 & methyl benzaldehyde & 15.51 & 120 & & & & & & & & & & & & & \\
\hline 73 & C4-benzene & 15.64 & 134 & & & & & & & 0.02 & & & & 0.40 & 0.24 & \\
\hline 74 & C4-benzene & 15.72 & 134 & 0.08 & 0.10 & 0.13 & & & & & & & & & 0.20 & \\
\hline 75 & A,A-dimethyl benzene methanol & 15.80 & 136 & & & & & & & & & & & & & \\
\hline 76 & C4-benzene & 15.86 & 134 & 0.14 & 0.19 & 0.29 & $0 . \overline{12}$ & & & & & & & 0.80 & 0.38 & \\
\hline 77 & 1-undecene & 15.95 & 154 & & & & & & & & & & & & & \\
\hline 78 & n-undecane & 16.05 & 156 & 0.17 & 0.24 & 0.31 & 0.16 & 0.03 & & & & & 0.44 & & 0.44 & 0.01 \\
\hline 79 & nonanal & 16.12 & 142 & 0.16 & 0.17 & & & 0.03 & & 0.07 & 0.01 & 0.83 & 0.52 & 0.26 & & \\
\hline 80 & C4-benzene & 16.24 & 134 & & & & & & & & & & 0.14 & 0.18 & & \\
\hline 81 & phosphoric acid, triethyl ester & 16.41 & 182 & & & 0.53 & & & & & & 0.31 & & & 0.39 & \\
\hline 82 & C4-benzene & 16.43 & 134 & 0.12 & & & 0.11 & & & & & & 0.20 & & & \\
\hline 83 & trans methyl decalin & 16.50 & 152 & & & & & & & & & & & & & \\
\hline 84 & C4-benzene & 16.55 & 134 & 0.12 & 0.14 & 0.23 & 0.09 & & & & & 0.24 & 0.08 & & 0.30 & \\
\hline 85 & cis methyl decalin & 16.74 & 152 & & & 0.14 & & & & & & & & & & \\
\hline 86 & C5-benzene & 16.79 & 148 & & & & & & & & & 0.43 & 0.11 & & & \\
\hline 87 & methyl indan & 17.14 & 132 & & & & & & & & & & 0.20 & 0.30 & & \\
\hline 88 & 0,0-diethyl-S-ethyl phosphorothioate & 17.16 & 198 & & 0.19 & 0.19 & 0.09 & & & & & & & & 0.23 & \\
\hline 89 & methy indan & 17.29 & 132 & & & & & & & & & & 0.21 & & & \\
\hline 90 & trichlorobenzene & 17.64 & 180 & & & 0.23 & & 0.01 & 0.28 & & & 0.82 & & 0.19 & 0.25 & \\
\hline 91 & 1-dodecene & 17.66 & 168 & & & & & & & & & 0.21 & & & & \\
\hline 92 & 1-(4-methylphenyl) ethanone & 17.67 & 134 & & & & & & & & & & & & & \\
\hline 93 & N,N-dibutyl-1-butanamine & 17.72 & 142 & & & & & & & & & & & & & \\
\hline 94 & n-dodecane & 17.80 & 170 & & & & & & & & & & 0.14 & 0.15 & & \\
\hline 95 & naphthalene & 17.79 & 128 & 0.21 & 0.12 & 0.33 & 0.15 & 0.05 & 0.01 & 0.04 & & 0.56 & 0.68 & 0.42 & 0.41 & 0.01 \\
\hline 96 & C5-benzene & 17.87 & 148 & & & & & & & & & & & & & \\
\hline 97 & decanal & 17.90 & 156 & & & & & 0.01 & & 0.01 & & & 0.67 & & & \\
\hline 98 & benzothiophene & 17.90 & 134 & & & & & & & & & & & & & \\
\hline 99 & 1,4-oxathiane, 4,4-dioxide & 18.35 & 136 & & & & & & & & & & & & & \\
\hline 100 & benzothiazole & 18.40 & 135 & & 0.09 & & & & & & & & & & & \\
\hline 101 & benzene propanenitrile & 18.53 & 131 & & & & & & & & & & & & & \\
\hline 102 & dimethyl indan & 18.80 & 146 & & & & & & & & & & & & & \\
\hline 103 & 3-tetradecene & 18.85 & 196 & & & & & & & & & & & & & \\
\hline 104 & $\mathrm{~N}$-butyl-N-nitroso $\mathrm{N}$-butanamine & 18.91 & 158 & & & & & & & & & & & & & \\
\hline 105 & 1-tridecene & 19.26 & 182 & & & & & & & & & & & & & \\
\hline 106 & n-tridecane & 19.38 & 184 & & & & & & & & & 0.16 & 0.14 & 0.11 & & \\
\hline 107 & $\mathrm{~N}, \mathrm{~N}$-dibutyl formamide & 19.51 & 157 & & 0.05 & 0.12 & & & & & & 0.33 & & & 0.09 & \\
\hline 108 & 2-methy naphthalene & 19.61 & 142 & 0.06 & 0.11 & 0.11 & 0.04 & 0.01 & & 0.02 & & 0.26 & 0.27 & 0.12 & 0.12 & \\
\hline 109 & phthalate & 19.80 & 390 & & & & & & & & & & & & & \\
\hline 110 & 1,3-isobenzofurandione & 19.81 & 148 & & & & & & & 0.03 & & 0.19 & 0.13 & & & \\
\hline 111 & 1-methyl naphthalene & 19.91 & 142 & & & & & & 0.02 & & & 0.16 & 0.21 & 0.10 & & \\
\hline 112 & 1-methyi-4-(propylthio) benzene & 20.37 & 166 & & & & & & & & & & & & & \\
\hline 113 & 1-tetradecene & 20.76 & 196 & & & & & & & & & & 0.20 & & & \\
\hline 114 & n-tetradecane & 20.86 & 198 & 0.05 & 0.07 & 0.05 & & 0.01 & & 0.01 & & & & & 0.12 & \\
\hline 115 & bipheny & 20.87 & 154 & & & & & 0.01 & 0.02 & & & 0.07 & 0.07 & & & \\
\hline 116 & 1,1'-oxy bis(benzene) & 21.17 & 186 & & & & & & 0.04 & & & 0.08 & 0.09 & & & \\
\hline 117 & $2,4,6$-trichloroaniline & 21.20 & 195 & & & & & 0.01 & & 0.01 & & 0.18 & 0.24 & & & \\
\hline 118 & C2-naphthalene & 21.28 & 156 & & & & & & & & & 0.17 & & & & \\
\hline 119 & C2-naphthalene & 21.51 & 156 & & & & & & 0.03 & & & & & & & \\
\hline 120 & C2-naphthalene & 21.56 & 156 & & & & & & & & & & & & & \\
\hline 121 & tribromobenzene & 21.62 & 312 & & & & & & 0.04 & & & 0.04 & & & & \\
\hline 122 & 6,10-dimethyl-5,9-undecadien-2-one & 21.65 & 194 & & & & & & & & & & & & & \\
\hline 123 & 2,6-di-t-butyl-2,5-cyclohexadiene-1,4-dione & 22.06 & 220 & & & & & & & & & 0.20 & & & & \\
\hline 124 & 1-pentadecene & 22.16 & 210 & & & & & & & & & & & & & \\
\hline 125 & n-pentadecane & 22.26 & 212 & & & & & & & & & & 0.06 & & & \\
\hline 126 & methyl biphenyl & 22.30 & 168 & & & & & & & & & & & & & \\
\hline 127 & 1,2-dihydroacenaphthylene & 22.50 & 168 & & & & & & 0.04 & & & & 1 & 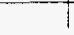 & $i$ & \\
\hline
\end{tabular}




\begin{tabular}{|c|c|c|c|c|c|c|c|c|c|c|c|c|c|c|c|c|}
\hline & & & & 25 & 24 & 23 & 26 & 124 & 123 & 141 & 142 & 146 & 147 & 178 & 21 & 127 \\
\hline & Compound & & & E129 & $E 129$ & E131 & E131 & E131 & $E 131$ & E131 & E131 & E131 & E131 & E131 & $E 132$ & E133 \\
\hline & Tentative Identification & RT & MW & $\mathrm{m} / \mathrm{e}$ & ne & $n$ & $\mathbf{s}$ & $\mathbf{s}$ & $n$ & s & $n w$ & $n w$ & ne & sump & $m$ & $\mathrm{~m}$ \\
\hline 128 & C3-naphthalene & 22.65 & 170 & & & & & & & & & & & & & \\
\hline 129 & phenyl maleic anhydride & 22.90 & 214 & & & & & & & 0.02 & & & & & & \\
\hline 130 & dibenzofuran & 22.93 & 168 & & & & & & 0.02 & & & & & & & \\
\hline 131 & \begin{tabular}{|l|} 
1 hexadecene \\
\end{tabular} & 22.47 & 224 & & & & & & & & & & & & & \\
\hline 132 & n-hexadecane & 23.57 & 226 & & & & & & & & & 0.09 & 0.02 & & & \\
\hline 133 & benzenedicarboxylic acid, diethyl ester & 23.64 & 222 & & & & & & & & & 0.10 & & & & \\
\hline 134 & 1,3-dibromo-2,2-bis(bromomethyl) propane & 24.17 & 384 & & & & & & 0.03 & & & & & & & \\
\hline 135 & \begin{tabular}{|l} 
diphenyl diazene \\
\end{tabular} & 24.23 & 182 & & & & & & 0.02 & & & & & & & \\
\hline 136 & benzophenone & 24.30 & 182 & & & & & & 0.01 & & & 0.15 & 0.06 & & & \\
\hline 137 & 1-heptadecene & 24.71 & 238 & & & & & & & & & & & & & \\
\hline 938 & n-heptadecane & 24.81 & 240 & & & & 0.04 & & & & & & & & & \\
\hline 139 & $\mathrm{~N}$-(phenyimethylene) benzamine & 24.83 & 181 & & & & & & & & & & & & & \\
\hline 140 & 9H-fluoren-9-one & 25.28 & 180 & & & & & & & & & & & & & \\
\hline 141 & tribromobenzamine & 25.39 & 327 & & & & & & 0.01 & & & & & & & \\
\hline 142 & trimethyl indan & 25.45 & 160 & & & & & & & & & & & & & \\
\hline 143 & dibenzothiophene & 25.58 & 184 & & & & & & & & & & & & & \\
\hline 144 & tetrabromobenzene & 25.86 & 390 & & & & & & 0.02 & & & & & & & \\
\hline 145 & In-octadecane & 25.88 & 254 & & & & 0.07 & & & & & & & & & \\
\hline 146 & phenanthrene/anthracene & 26.37 & 178 & 0.06 & 0.07 & 0.15 & 0.44 & 0.01 & 0.01 & & & 0.32 & 0.15 & & & \\
\hline 147 & methyl dibenzothiophene & 26.98 & 198 & & & & & & & & & & & & & \\
\hline 148 & n-nonadecane & 27.04 & 268 & & & & 0.12 & & & & & & & & & \\
\hline 149 & 2-methyl anthracene/phenanthrene & 27.48 & 192 & & & & & & & & & & & & & \\
\hline 150 & 1-methyl anthracene/phenanthrene & 27.57 & 192 & & & & 0.25 & & & & & & & & & \\
\hline 151 & 2,6-dibutyl-2,5-cyclohexadien-1,4-dione & 31.30 & 220 & & & & & & & 0.05 & & & & 1.71 & & \\
\hline 152 & Total PCB's & & & & & 0.30 & 7.30 & & & & 1.60 & & 1.50 & 0.30 & & \\
\hline & & & & & & & & & & & & & & & & \\
\hline & & & & & & & & & & & & & & & & \\
\hline & & & & & & & & & & & & & & & & \\
\hline & & & & & & & & & & & & & & & & \\
\hline & & & & & & & & & & & & & & & & \\
\hline & & & & & & & & & & & & & & & & \\
\hline & & & & & & & & & & & & & & & & \\
\hline & & & & & & & & & & & & & & & & \\
\hline & & & & & & & & & & & & & & & & \\
\hline & & & & & & & & & & & & & & & & \\
\hline & & & & & & & & & & & & & & & & \\
\hline & & & & & & & & & & & & & & & & \\
\hline & & & & & & & & & & & & & & & & \\
\hline & & & & & & & & & & & & & & & & \\
\hline & & & & & & & & & & & & & & & & \\
\hline & & & & & & & & & & & & & & & & \\
\hline & & & & & & & & & & & & & & & & \\
\hline & & & & & & & & & & & & & & & & \\
\hline & & & & & & & & & & & & & & & & \\
\hline & & & & & & & & & & & & & & & & \\
\hline & & & & & & & & & & & & & & & & \\
\hline & & & & & & & & & & & & & & & & \\
\hline & & & & & & & & & & & & & & & $\ldots$ & \\
\hline & & & & & & & & & & & & & & $\ldots$ & & \\
\hline & & & & & & & & & & & & & & & & \\
\hline & & & & & & & & & & & & & & & & \\
\hline & & & & & & & & & & & & & & & & \\
\hline & & & & & & & & & & & & & & & & \\
\hline & & & & & & & & & & & & & & & 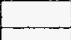 & \\
\hline & & & & & & & & & & & & & & & & \\
\hline & & & & & & & & & & & & & & & & \\
\hline & & & & & & & & & & & & & & & & \\
\hline & & & & & & & & & & & & & & & & \\
\hline & & & & & & & & & & & & & & & & \\
\hline & - & & & & & & & & & & & & & & & \\
\hline & ( & 1 & 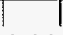 & . & & 11 & 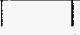 & 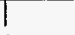 & 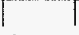 & 7 & & & & & 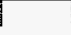 & \\
\hline
\end{tabular}




\begin{tabular}{|c|c|c|c|c|c|c|c|c|c|c|c|c|c|c|c|c|}
\hline & & 143 & 1 & 2 & 22 & 144 & 128 & 129 & 140 & 138 & 46 & 33 & 32 & 30 & 31 & 36 \\
\hline & Compound & E133 & E134 & E134 & E134 & E134 & E134 & & E135 & $E 200$ & E201 & E203 & E203 & E204 & $E 204$ & E205 \\
\hline & Tentative Identification & $\mathbf{m}$ & se & $n w$ & $\mathrm{~m}$ & $m$ & $\mathbf{m}$ & 0 & & $\mathbf{m}$ & $m$ & $\mathbf{n}$ & s & $n$ & $\mathbf{s}$ & $\mathrm{m}$ \\
\hline 1 & sulfur dioxide & & & & & & & & & & & & & & & \\
\hline 2 & 2-propanone & & & 2.60 & & & & & & & & & & & & \\
\hline 3 & 1-fluoro-1,1-dichloroethane & & 1.75 & & & & & & & & & & & & & \\
\hline 4 & N-ethyl ethanamine & & & & & 0.14 & & & & & & & & & & \\
\hline 5 & 2-butanone & & & & & & & & & & & & & & & \\
\hline 6 & $\mathrm{~N}$-ethyl-N-methyl ethanamine & & & & & & & & & & & & & & & \\
\hline 7 & dichloromethane & & & & & & & & & & & & & & & \\
\hline 8 & $\mathrm{~N}-(1$-methyle(hy) $\}$-2-propanamine & 0.13 & 0.69 & & 2.68 & 0.22 & & & 0.12 & & & & & & & \\
\hline 9 & benzene & & & & & & & & & & & 0.01 & & 0.01 & 0.01 & 0.02 \\
\hline 10 & 2-pentanone & & & & & & & & & & & & & & & \\
\hline 11 & trichloroethene & 0.04 & & & 0.41 & & & & & & & & & & & \\
\hline 12 & $\mathrm{~N}$-(1-methylethylidene)-2-propanamine & & & & & & & & & & & & & & & \\
\hline 13 & toluene & 0.05 & 9.46 & 1.01 & 2.20 & 0.11 & & & 0.08 & 0.04 & & 0.36 & 0.28 & 0.28 & 0.33 & 0.06 \\
\hline 14 & $\mathrm{~N}$-methyl-N-(1-methylethyl)-2-propanamine & & & & & & & & & & & & & & & \\
\hline 15 & 3-hexanone & & & & & & & & & & & & & & & \\
\hline 16 & 2-hexanone & & & & & & & & & & & & & & & \\
\hline 17 & n-octane & & & & & & & & & & & & & & & \\
\hline 18 & hexanal & & & & & & & & & & & & & & & \\
\hline 19 & tetrachloroethene & 0.05 & & & 1.05 & 0.07 & & & & & & 0.03 & 0.02 & 0.01 & 0.02 & 0.02 \\
\hline 20 & methyl ethyl disulfide & & & & & & & & & & & & & & & \\
\hline 21 & chlorobenzene & & & & & & & & & & & & & & & \\
\hline 22 & ethyl benzene & & 0.33 & 0.26 & 1.03 & & & & & & & 0.04 & 0.02 & 0.03 & 0.03 & 0.01 \\
\hline 23 & e.m-xylene & 0.14 & 2.32 & 1.87 & 6.88 & 0.25 & 0.02 & & 0.08 & & & 0.17 & 0.13 & 0.15 & 0.16 & 0.05 \\
\hline 24 & 1,4 oxathiane & 0.02 & 0.48 & 0.47 & 1.74 & 0.10 & & & & & & & & & & 0.00 \\
\hline 25 & styrene & & 0.22 & 0.11 & & & & & & & & 0.02 & 0.02 & 0.02 & 0.03 & \\
\hline 26 & 3-heptanone & & & & & & & & & & & & & & & \\
\hline 27 & o-xylene & 0.07 & 1.10 & 0.87 & 3.77 & 0.13 & 0.01 & & 0.03 & & & 0.07 & 0.05 & 0.05 & 0.03 & 0.03 \\
\hline 28 & heptanal & & & & & & & & & & & & & & & \\
\hline 29 & n-nonane & & & 0.11 & 0.33 & & & & & & & 0.04 & 0.02 & 0.02 & & 0.02 \\
\hline 30 & C3-benzene & & & & & & & & & & & & & & & \\
\hline 31 & N-butylidene-1-butanamine & & & & & & & & & & & & & & & \\
\hline 32 & C3-benzene & & & & 0.29 & & & & & & & & & & & \\
\hline 33 & 3-methyl nonane & & & & & & & & & & & & & & & \\
\hline 34 & pinene & & & & & & & & & & & & & & & \\
\hline 35 & 2-methyl cyclohexanol & & & & & & & & & & & & & & & \\
\hline 36 & 3-octanone & & 0.24 & 0.18 & 0.63 & & & & & & & & & & & \\
\hline 37 & 2ethyl hexanal & & & & & & & & & & & & & & & \\
\hline 38 & 1-chloro-2-methyl benzene & & & & & & & & & & & & & & & \\
\hline 39 & propyl benzene & & 0.21 & 0.15 & 0.60 & & & & & & & 0.03 & & 0.01 & & \\
\hline 40 & N-butyl-1-butanamine & & & & & 0.07 & & & & & & & & & & \\
\hline 41 & benzaidehyde & 0.06 & 0.97 & 0.55 & & 0.07 & & 0.06 & & 0.05 & 0.03 & 0.07 & 0.05 & 0.07 & 0.06 & 0.08 \\
\hline 42 & C3-benzene & 0.01 & 0.81 & 0.45 & 2.47 & & & & & & & 0.11 & 0.08 & 0.10 & 0.10 & 0.02 \\
\hline 43 & C3-benzene & 0.03 & 0.59 & 0.39 & 1.49 & 0.05 & & & & & & 0.05 & 0.04 & 0.03 & 0.05 & \\
\hline 44 & phenol & 0.02 & & & & 0.03 & & & & 0.01 & & & & 0.08 & & \\
\hline 45 & aniline & & & & & & & & & & & & & & & \\
\hline 46 & C3-benzene & 0.06 & & & 0.66 & 0.12 & & & & & & & & & 0.16 & \\
\hline 47 & 6-methyl-5-hepten-2-ane & & & & & & & & & & & & & & & \\
\hline 48 & benzonitrile & & 0.98 & 0.70 & & & & & & & & & & & & \\
\hline 49 & 1-decene & & & & & & & & & & & & & & & \\
\hline 50 & C3-benzene & & & & 4.43 & & & & 0.04 & & & & & & & \\
\hline 51 & n-decane & 0.06 & 0.64 & 0.46 & 1.36 & 0.08 & & & 0.02 & & & 0.16 & 0.11 & 0.15 & 0.12 & 0.07 \\
\hline 52 & octanal & & & & & & & & & & & & & & & \\
\hline 53 & dichlorobenzene & & & 0.23 & trace & & & & & & & & & 0.02 & & \\
\hline 54 & 4-methyl decane & & 0.15 & 0.16 & 0.62 & & & & & & & & & 0.01 & & \\
\hline 55 & C3-benzene & 0.03 & & 0.86 & & 0.06 & & & 0.02 & & & & & 0.03 & & \\
\hline 56 & C4-benzene & & 0.94 & & & & & & & & & & & & & \\
\hline 57 & 2-ethyl hexanol & 0.06 & & & 4.48 & 0.14 & 0.01 & & 0.11 & & 0.07 & 0.14 & 0.10 & & 0.08 & 0.11 \\
\hline 58 & limonene & & 0.07 & 0.12 & & & & & & & 0.10 & & & 0.01 & 0.12 & 0.03 \\
\hline 59 & benzene methanol & & 0.16 & & 0.72 & & & & & & & & & 0.07 & & \\
\hline 60 & indan & & & & & & & & & & & & & & & \\
\hline 61 & dichlorobenzene & & & 0.24 & 1.04 & & & & & & & & & & & \\
\hline 62 & $\mathrm{~N}$-ethyl-N-(1-methylethyl)-2-propanamine & & 0.19 & & & & & & & & & & & & & \\
\hline 63 & C4-benzene & 0.01 & 0.21 & 0.31 & 0.71 & 0.04 & & & ( & & & & & & & \\
\hline
\end{tabular}


Air Monitoring Results

Sample number and room number at the top of each data column

\begin{tabular}{|c|c|c|c|c|c|c|c|c|c|c|c|c|c|c|c|c|}
\hline & & 143 & 1 & 2 & 22 & 144 & 128 & 129 & 140 & 138 & 46 & 33 & 32 & 30 & 31 & 36 \\
\hline & Compound & E133 & E134 & $E 134$ & $\mathrm{E} 134$ & $E 134$ & E134 & & E135 & E200 & E201 & E203 & E203 & E204 & E204 & E205 \\
\hline & Tentative Identification & $m$ & se & $n w$ & $m$ & $m$ & $m$ & 0 & & $\mathbf{m}$ & $\mathrm{m}$ & $n$ & $\mathbf{s}$ & $n$ & $\mathbf{s}$ & $m$ \\
\hline 64 & 5-methyi decane & & 0.15 & 0.11 & 0.45 & & & & & & & & & 0.02 & & \\
\hline 65 & 4-methyi decane & & & & & & & & & & & & & & & \\
\hline 66 & C4-benzene & & 0.28 & 0.21 & 1.00 & & & & & & & & & & & \\
\hline 67 & 2-methyl decane & & 0.21 & 0.42 & 0.45 & & & & & & & & & 0.02 & & \\
\hline 68 & 1,4-dithiane & & & & 2.06 & 0.05 & & & & & & & & & & \\
\hline 69 & acetophenone & 0.05 & & & & 0.06 & & 0.04 & & 0.03 & & & & 0.11 & & \\
\hline 70 & C4-benzene & & 0.48 & & & & & & & & & & & & & \\
\hline 71 & 3-methyl decane & & & & & & & & & & & & & & & \\
\hline 72 & methyl benzaldehyde & & & & & & & & & & & & & & & \\
\hline 73 & C4-benzene & & & & & & & & & & & & & & & \\
\hline 74 & C4-benzene & & & & 1.04 & & & & & & & & & & & \\
\hline 75 & A.A-dimethyl benzene methanol & & & & & & & & & & & & & & & \\
\hline 76 & C4-benzene & & 0.26 & 0.14 & 0.94 & 0.01 & & & & & & & & & 0.08 & \\
\hline 77 & 1-undecene & & & & & & & & & & & & & & & \\
\hline 78 & n-undecane & & 0.34 & 0.21 & 1.10 & & & & 0.02 & & & 0.20 & 0.15 & 0.17 & 0.14 & 0.06 \\
\hline 79 & nonanal & 0.06 & & & & 0.08 & & & & 0.02 & & 0.08 & 0.04 & 0.09 & 0.08 & 0.05 \\
\hline $\mathrm{BO}$ & C4-benzene & & & & 0.39 & & & & & & & & & & & \\
\hline 81 & phosphoric acid, triethyl ester & & & & 1.80 & & & & & & & & & & & \\
\hline 82 & C4-benzene & & 0.17 & 0.13 & & & & & & & & & & & & \\
\hline 83 & trans methyt decalin & & & & & & & & & & & & & & & \\
\hline 84 & C4-benzene & & 0.23 & 0.11 & 0.72 & & & & & & & & & 0.02 & & \\
\hline 85 & cis methyl decalin & & & & 0.49 & & & & & & & & & & & \\
\hline 86 & C5-benzene & & & & & & & & & & & & & & & \\
\hline 87) & methy indan & & & & & & & & & & & & & & & \\
\hline B8 & 0,0 -diethyl-S-ethyl phosphorothioate & & & & 0.63 & & & & & & & & & & & \\
\hline 89 & methyl indan & & & & & & & & & & & & & & & \\
\hline 90 & trichlorobenzene & & & 0.04 & 0.38 & 0.01 & & & & & & & & & & \\
\hline 91 & 1-dodecene & & & & & & & & & & & & & & & \\
\hline 92 & 1-(4-methylpheny) ethanone & & & & 0.22 & & & & & & & & & & & \\
\hline 93 & $\mathrm{~N}, \mathrm{~N}$-dibutyl-1-butanamine & & & & & & & & & & & & & & & \\
\hline 94 & n-dodecane & & & & & & & & & & & & & & & \\
\hline 95 & naphthalene & 0.02 & 0.35 & 0.26 & 0.99 & 0.05 & & & 0.01 & 0.01 & & 0.21 & 0.15 & 0.16 & 0.16 & 0.08 \\
\hline 96 & C5-benzene & & & & & & & & & & & & & & & \\
\hline 97 & decanal & 0.04 & & & & 0.04 & & & & & & & & 0.03 & & 0.03 \\
\hline 98 & benzothiophene & & & & & & & & & & & & & & & \\
\hline 99 & 1,4-oxathiane, 4,4-dioxide & & & & & 0.02 & & & & & & & & & & \\
\hline 100 & benzothiazole & & & & 0.33 & & & & & & & & & & & \\
\hline 101 & benzene propanenitrile & & & & & & & & & & & & & & & \\
\hline 102 & dimethy indan & & & & & & & & & & & & & & & \\
\hline 103 & 3-tetradecene & & 0.19 & 0.11 & & & & & & & & & & & & \\
\hline 104 & $\mathrm{~N}$-butyl- $\mathrm{N}$-nitroso $\mathrm{N}$-butanamine & & & & 0.32 & & & & & & & & & & & \\
\hline 105 & 1-tridecene & & & & & & & & & & & & & & & \\
\hline 106 & n-tridecane & & & & 0.23 & & & & & & & & & & & \\
\hline 107 & N,N-dibutyl formamide & & 0.25 & 0.18 & 0.56 & 0.03 & & & & & & & & & & \\
\hline 108 & 2-methyl naphthalene & 0.00 & 0.19 & 0.08 & 0.50 & 0.02 & & & & & & & 0.05 & 0.04 & 0.02 & \\
\hline 109 & phthaiate & & & & & & & & & & & & & & 0.03 & \\
\hline 110 & 1,3-isobenzofurandione & & & & & & & & & & & & & & & \\
\hline 111 & 1 -methyl naphthalene & & & & 0.39 & 0.03 & & & & & & & & & & \\
\hline 112 & 1-methyl-4-(propylthio) benzene & & & & & & & & & & & & & & & \\
\hline 113 & 1-tetradecene & & & & & & & & & & & & & & & \\
\hline 114 & n-tetradecane & & 0.11 & 0.11 & 0.17 & & & & & & & & & & & \\
\hline 115 & bipheny & & & & & & & & & 0.01 & & 0.09 & 0.07 & 0.09 & 0.08 & 0.07 \\
\hline 116 & 1,1'-oxy bis(benzene) & & & & & & & & & 0.02 & & & & 0.01 & 0.06 & \\
\hline 117 & 2,4,6-trichloroaniline & & & & & 0.01 & & & & & & 0.04 & 0.04 & 0.09 & 0.07 & \\
\hline 118 & C2-naphthalene & & & & & & & & & & & & & & & \\
\hline 119 & C2-naphthalene & & & & & & & & & & & & & & & \\
\hline 120 & C2-naphthaiene & & & & & & & & & & & & & & & \\
\hline 121 & tribromobenzene & & & & & & & & & & & & & & & \\
\hline 122. & 6,10-dimethyl-5,9-undecadien-2-one & & & & & & & & & & & & & & & \\
\hline 123 & 2,6-di-t-butyl-2,5-cyclohexadiene-1,4-dione & & & & & & & & & & & & & & & \\
\hline 124 & 1-pentadecene & & & & & & & & & & & & & & & \\
\hline 125 & n-pentadecane & & 0.09 & 0.11 & & & & & & & & & & & & \\
\hline 126 & methyl biphenyl & & & & & & & & & & & & & & & \\
\hline 127 & 1,2-dihydroacenaphthylene & & & & & & & & & & & & & & & \\
\hline
\end{tabular}


Air Monitoring Results

Sample number and room number at the top of each data column

\begin{tabular}{|c|c|c|c|c|c|c|c|c|c|c|c|c|c|c|c|c|}
\hline & & 143 & 1 & 2 & 22 & 144 & 128 & 129 & 140 & 138 & 46 & 33 & 32 & 30 & 31 & 36 \\
\hline & Compound & E133 & E134 & E134 & E134 & E134 & $E 134$ & & E135 & $E 200$ & E201 & E203 & E203 & E204 & E204 & E205 \\
\hline & Tentalive Identification & $m$ & se & $n w$ & $\mathbf{m}$ & $m$ & $m$ & a & & $\dot{m}$ & $\mathrm{~m}$ & $n$ & s & $n$ & $\mathbf{s}$ & m \\
\hline 128 & C3-naphthalene & & & & & & & & & & & & & & & \\
\hline 129 & phenyl maleic anhydride & & & & & & & & & & & & & & & \\
\hline 130 & dibenzofuran & & & & & & & & & & & & & & & \\
\hline 131 & 1-hexadecene & & & & & & & & & & & & & & & \\
\hline 132 & n-hexadecane & & & & & & & & & & & & & & & \\
\hline 133 & benzenedicarboxylic acid, diethy ester & & & & & & & & & & & & & & & \\
\hline 134 & 1,3-dibromo-2,2-bis(bromomethyl) propane & & & & & & & & & & & & & & & \\
\hline 135 & diphenyl diazene & & & & & & & & & & & & & & & \\
\hline 136 & benzophenone & & & & & & & & & & & & & & & \\
\hline 137 & 1-heptadecene & & & & & & & & & & & & & & & \\
\hline 138 & n-heptadecane & & & & & & & & & & & & & & & \\
\hline 139 & $\mathrm{~N}$-(phenylmethylene) benzamine & & & & & & & & & & & & & & & \\
\hline 140 & 9H-fluoren-9-one & & & & & & & & & & & & & & & \\
\hline 141 & tribromobenzamine & & & & & & & & & & & & & & & \\
\hline 142 & trimethyl indan & & & & & & & & & & & & & & & \\
\hline 143 & dibenzothiophene & & & & & & & & & & & & & & & \\
\hline 144 & tetrabromobenzene & & & & & & & & & & & & & & & \\
\hline 145 & n-octadecane & & & & & & & & & & & & & & & \\
\hline 146 & phenanthrene/anthracene & 0.01 & & & & 0.01 & & & & & 0.03 & 0.02 & & & & 0.02 \\
\hline 147 & methyl dibenzothiophene & & & & & & & & & & & & & & & \\
\hline 148 & n-nonadecane & & & & & & & & & & & & & & & \\
\hline 149 & 2-methyl anthracene/phenanthrene & & & & & & & & & & & & & & & \\
\hline 150 & 1 -methyl anthracene/phenanthrene & & & & & & & & & & & & & & & \\
\hline 151 & 2,6-dibuty|-2,5-cyclohexadien-1,4-dione & & & & & & & & & & & & & & & \\
\hline 152 & Total PCB's & & & & & & & & & & 0.70 & & 0.50 & 0.50 & & 0.40 \\
\hline & & & & & & & & & & & & & & & & \\
\hline & & & & & & & & & & & & & & & & \\
\hline & & & & & & & & & & & & & & & & \\
\hline & & & & & & & & & & & & & & & & \\
\hline & & & & & & & & & & & & & & & & \\
\hline & & & & & & & & & & & & & & & & \\
\hline & & & & & & & & & & & & & & & & \\
\hline & & & & & & & & & & & & & & & & \\
\hline & & & & & & & & & & & & & & n & & \\
\hline & & & & & & & & & & & & & & & & \\
\hline & & & & & & & & & & & & & & & & \\
\hline & & & & & & & & & & & & & & & & \\
\hline & & & & & & & & & & & & & & & & \\
\hline & & & & & & & & & & & & & & & & \\
\hline & & & & & & & & & & & & & & & & \\
\hline & & & & & & & & & & & & & & & & \\
\hline & & & & & & & & & & & & & & & & \\
\hline & & & & & & & & & & & & & & & & \\
\hline & & & & & & & & & & & & & & & & \\
\hline & & & & & & & & & & & & & & & & \\
\hline & & & & & & & & & & & & & & & & \\
\hline & & & & & & & & & & & & & & & & \\
\hline & & & & & & & & & & & & & & & & \\
\hline & & & & & & & & & & & & & & & & \\
\hline & & & & & & & & & & & & & & & & \\
\hline & & & & & & & & & & & & & & & & \\
\hline & & & & & & & & & & & & & & & & \\
\hline & & & & & & & & & & & & & & & & \\
\hline & & & & & & & & & & & & & & & & \\
\hline & & & & & & & & & & & & & & & & \\
\hline & & & & & & & & & & & & & & & & \\
\hline & & & & & & & & & & & & & & & & \\
\hline & & & & & & & & & & & & & & & & \\
\hline & & & & & & & & & & & & & & & & \\
\hline & & & & & & & & & & & & & & & & \\
\hline & & & & & & & & & & & & & & & & \\
\hline & & & & & & & & & & & & & & & & \\
\hline & 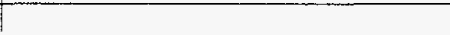 & (2. & 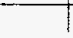 & . & 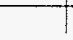 & ! & 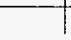 & . & & ( & & & & & & \\
\hline
\end{tabular}




\begin{tabular}{|c|c|c|c|c|c|c|c|c|c|c|c|c|c|c|c|c|}
\hline & Compound & & & $\begin{array}{c}37 \\
E 207\end{array}$ & \begin{tabular}{|c|}
163 \\
$E 207$ \\
\end{tabular} & \begin{tabular}{|c|}
35 \\
$E 208$ \\
\end{tabular} & \begin{tabular}{|c|}
34 \\
$E 208$ \\
\end{tabular} & $\begin{array}{c}38 \\
E 209\end{array}$ & \begin{tabular}{|c|}
39 \\
$E 211$ \\
\end{tabular} & \begin{tabular}{|c|}
42 \\
$E 213$ \\
\end{tabular} & $\begin{array}{c}43 \\
E 214\end{array}$ & \begin{tabular}{|c|}
44 \\
$E 215$ \\
\end{tabular} & \begin{tabular}{|c|}
41 \\
$E 216$ \\
\end{tabular} & \begin{tabular}{|c|}
40 \\
$E 216$ \\
\end{tabular} & \begin{tabular}{|c|}
45 \\
$E 217$ \\
\end{tabular} & $\frac{47}{E 220}$ \\
\hline & Tentative Identification & RT & MW & $\mathrm{m}$ & $\mathrm{m}$ & $\mathrm{n}$ & s & opbal & clost & $m$ & $\mathrm{~m}$ & $\mathrm{~m}$ & $n$ & $\mathrm{~s}$ & $\mathrm{~m}$ & se \\
\hline 1 & sulfur dioxide & 1.55 & 64 & & & & & & & & & & & & & \\
\hline 2 & 2-propanone & 1.89 & 58 & & & & & & & & & & & & & \\
\hline 3 & 1-fluoro-1,1-dichloroethane & 1.95 & 116 & & & & & & & & & & & & & \\
\hline 4 & N-ethyi ethanamine & 2.36 & 73 & & & & & & & & & & & & & \\
\hline 5 & 2-butanone & 2.56 & 72. & & & & & & & & & & & & & \\
\hline 6 & $\mathrm{Nethyl-N-methyl} \mathrm{ethanamine}$ & 2.94 & 87 & & & & & & & & & & & & & \\
\hline 7 & dichtoromethane & 3.15 & 84 & 0.04 & & & & & & & & & & & & \\
\hline 8 & $\mathrm{~N}$-(1-methylethyl)-2-propanamine & 3.59 & 101 & & & & & & & & & & & & & \\
\hline 9 & benzene & 3.64 & 78 & & 2.53 & 0.01 & 0.01 & 0.02 & & 0.01 & 0.02 & 0.03 & & 0.01 & 0.08 & 0.15 \\
\hline 10 & 2-pentanone & 4.08 & 86 & & & & & & & & & & & & & \\
\hline 11 & trichloroethene & 4.47 & \begin{tabular}{|c|}
130 \\
\end{tabular} & & 1.19 & & & & & & & & & & & \\
\hline 12 & $\mathrm{~N}$-(1-melhylethylidene)-2-propanamine & 4.62 & 99 & & & & & & & & & & & & & \\
\hline 13 & toluene & 6.97 & 92 & 0.15 & 6.25 & 0.01 & 0.13 & 0.06 & 0.05 & 0.05 & 0.07 & 0.65 & 0.06 & 0.06 & 1.11 & 0.97 \\
\hline 14 & $\mathrm{~N}$-methyl-N-(1-methylethy)-2-propanamine & 7.27 & 115 & & & & & & & & & & & & & \\
\hline 15 & 3-hexanone & 7.75 & 100 & & & & & & & & & & & & & \\
\hline 16 & 2-hexanone & 7.92 & 100 & & & & & & & & & & & & & \\
\hline 17 & n-octane & 8.29 & 114 & & 0.30 & & & & & & & & & & & \\
\hline 18 & hexanal & 8.33 & 100 & & & & & & & & & & & & & \\
\hline 19 & tetrachloroethene & 8.79 & 164 & 0.02 & 1.59 & 0.01 & 0.03 & 0.01 & 0.01 & 0.01 & 0.02 & 0.05 & 0.01 & 0.01 & 0.08 & 0.04 \\
\hline 20 & methyl ethyl disulfide & 9.68 & 108 & & & & & & & & & & & & & \\
\hline 21 & chlorobenzene & 10.05 & 112 & & & & & & & & & & & & & \\
\hline 22 & ethyl benzene & 10.57 & 106 & 0.02 & 1.28 & 0.02 & 0.02 & 0.01 & 0.01 & 0.01 & 0.01 & 0.12 & 0.01 & 0.01 & 0.23 & 0.16 \\
\hline 23 & $\mathrm{p}, \mathrm{m}$-xylene & 10.81 & 106 & 0.05 & 3.44 & 0.05 & 0.05 & 0.01 & 0.04 & 0.02 & 0.03 & 0.42 & 0.05 & 0.03 & 0.76 & 0.51 \\
\hline 24 & $1,4-0 \times a$ athiane & 11.00 & 104 & & & & & & & & & & & & & \\
\hline 25 & styrene & 11.30 & 118 & & 0.46 & & 0.01 & 0.02 & & & & 0.04 & & 0.01 & 0.09 & 0.08 \\
\hline 26 & 3-heptanone & 11.37 & 114 & & & & & & & & & & & & & \\
\hline 27 & o-xylene & 11.49 & 906 & 0.03 & 1.50 & 0.03 & 0.03 & 0.01 & 0.02 & 0.01 & 0.01 & 0.15 & 0.02 & 0.01 & 0.28 & 0.19 \\
\hline 28 & heptanal & 11.60 & 114 & & & & & & & & & & & & & \\
\hline 29 & n-nonane & 11.67 & 128 & 0.01 & & & 0.03 & 0.04 & & & & 0.02 & 0.02 & 0.01 & 0.04 & 0.03 \\
\hline 30 & C3-benzene & 12.20 & 120 & & & & & & & & & & & & & \\
\hline 31 & N-butylidene-1-butanamine & 12.28 & 127 & & & & & & & & & & & & & \\
\hline 32 & C3-benzene & 12.33 & 120 & & & & & & & & & & & & & \\
\hline 33 & 3-methyi nonane & 12.51 & 142 & & & & & & & & & & & & & \\
\hline 34 & pinene & 12.61 & 136 & & 0.21 & & & & & & - & 0.05 & & & 0.08 & 0.09 \\
\hline 35 & 2-methyl cyciohexanol & 12.62 & 114 & & & & & & & & & & & & & \\
\hline 36 & 3-octanone & 12.71 & 128 & & & & & & & & & & & & & \\
\hline 37 & 2-ethyl hexanal & 13.02 & 128 & & & & & & & & & & & & & \\
\hline 38 & 1-chloro-2-methyl benzene & 13.03 & 126 & & & & & & & & & & & & & \\
\hline 39 & propyl benzene & 13.05 & \begin{tabular}{|l|}
120 \\
\end{tabular} & & 0.66 & & & & & & & 0.05 & & & 0.07 & 0.04 \\
\hline 40 & N-butyl-1-butanamine & 13.17 & 129 & & & & & & & & & & & & & \\
\hline 41 & benzaldehyde & 13.18 & 106 & 0.09 & & 0.06 & 0.07 & 0.16 & 0.05 & 0.06 & 0.07 & 0.05 & 0.06 & 0.08 & 0.06 & 0.12 \\
\hline 42 & C3-benzene & 13.25 & 120 & & 2.61 & & & & & & & 0.18 & 0.01 & & 0.31 & 0.21 \\
\hline 43 & C3-benzene & 13.41 & 120 & & 0.52 & & & & & & & 0.06 & & & 0.10 & 0.08 \\
\hline 44 & phenol & 13.57 & 94 & & & & & & & & & & & & & \\
\hline 45 & aniline & 13.61 & 93 & & & & & & & & & & & & & \\
\hline 46 & C3-benzene & 13.68 & 120 & & 0.89 & & & & & & & & & & & \\
\hline 47 & 6-methyl-5-hepten-2-one & 13.69 & \begin{tabular}{|l|l|}
126 \\
\end{tabular} & & & & & & & & & & & & & \\
\hline 48 & benzonitrile & 13.72 & \begin{tabular}{|l|}
103 \\
\end{tabular} & 0.08 & & & & & & & & & & 0.07 & & \\
\hline 49 & 1-decene & 13.83 & 140 & & & & & & & & & & & & & \\
\hline 50 & C3-benzene & 93.99 & 120 & & & & & & & & & & & & & \\
\hline 51 & n-decane & 14.07 & \begin{tabular}{|l|l|}
142 \\
\end{tabular} & 0.09 & 0.52 & 0.06 & 0.06 & & 0.07 & 0.08 & & 0.09 & 0.04 & 0.05 & 0.11 & 0.08 \\
\hline 52 & Octanal & 14.09 & 128 & & & & & & & & & & & & & \\
\hline 53 & $\begin{array}{l}\text { dichlorobenzene } \\
\end{array}$ & 14.40 & \begin{tabular}{|l|}
146 \\
\end{tabular} & & 0.44 & & & & & & & 0.02 & & & 0.04 & \\
\hline 54 & 4-methyl decane & 14.56 & 156 & & 0.16 & & & & & & & & & & & \\
\hline 55 & C3-benzene & 14.57 & 120 & & 0.48 & & & & & & & & & & & \\
\hline 56 & C4-benzene & 14.60 & 134 & & 0.19 & & & & & & & & & & & \\
\hline 57 & 2ethyl hexanol & 14.64 & 130 & 0.09 & & 0.04 & 0.15 & 0.06 & 0.04 & 0.04 & & 0.14 & 0.06 & 0.11 & & \\
\hline 58 & limonene & 14.75 & 136 & & & & & 0.01 & 0.03 & 0.01 & 0.03 & 0.11 & 0.01 & 0.01 & 0.16 & 0.09 \\
\hline 59 & benzene methanol & 14.77 & 108 & & & & & & & & & & & & & \\
\hline 60 & indan & 14.87 & 118 & & 0.32 & & & & & & & & & & & \\
\hline 61 & dichlorobenzene & 14.89 & 146 & & & & & & & & & & & & & \\
\hline 62 & $\mathrm{~N}$-ethyl-N-(1-methylethyl)-2-propanamine & 15.07 & 129 & & & & & & & & & & & & & \\
\hline 63 & C4-benzene & 15.10 & $\mid 134$ & & 0.70 & & & & & & & & & & & \\
\hline
\end{tabular}




\begin{tabular}{|c|c|c|c|c|c|c|c|c|c|c|c|c|c|c|c|c|}
\hline & & & & 37 & 163 & 35 & 34 & 38 & 39 & 42 & 43 & 44 & 41 & 40 & 45 & 47 \\
\hline & Compound & & & $E 207$ & E207 & E208 & E208 & E209 & E211 & E213 & E214 & E215 & E216 & E216 & $E 217$ & $E 220$ \\
\hline & Tentative Identification & RT & $M W$ & $m$ & $\mathbf{m}$ & $n$ & $\mathbf{s}$ & o,bal & clost & $m$ & $m$ & $m$ & $n$ & $s$ & $m$ & se \\
\hline 64 & 5-methyl decane & 15.21 & 156 & & & & & & & & & & & & & \\
\hline 65 & 4-methyl decane & 15.29 & 156 & & & & & & & & & & & & & \\
\hline 66 & C4-benzene & 15.30 & 134 & & & & & & & & & & & & & \\
\hline 67 & 2-methyi decane & 15.34 & 156 & & & & & & & & & & & & & \\
\hline 68 & 1,4-dithiane & 15.39 & 120 & & & & & & & & & & & & & \\
\hline 69 & acetophenone & 15.47 & 120 & & 1.02 & & & 0.11 & & & & & & & & \\
\hline 70 & C4-benzene & 15.47 & 134 & & & & & & & & & & & & & \\
\hline 71 & 3-methyl decane & 15.48 & 156 & & & & & & & & & & & & & \\
\hline 72 & methyl benzaldehyde & 15.51 & 120 & & & & & & & & & & & & & \\
\hline 73 & C4-benzene & 15.64 & 134 & & 0.35 & & & & & & & & & & & \\
\hline 74 & C4-benzene & 15.72 & 134 & & 0.78 & & & & & & & & & & & \\
\hline 75 & A,A-dimethyl benzene methanol & 15.80 & 136 & & & & & & & & & & & & & \\
\hline 76 & C4-benzene & 15.86 & 134 & & & & & & & & & & & & & \\
\hline 77 & 1-undecene & 15.95 & 154 & & & & & & & & & & & & & \\
\hline 78 & n-undecane & 16.05 & 156 & 0.07 & & 0.03 & & & 0.02 & & & 0.06 & 0.03 & 0.02 & 0.11 & 0.07 \\
\hline 79 & nonanal & 16.12 & 142 & 0.05 & 0.15 & 0.06 & & 0.10 & 0.08 & 0.12 & 0.08 & & 0.08 & 0.04 & & \\
\hline 80 & C4tbenzene & 16.24 & 134 & & & & & & & & & & & & & \\
\hline 81 & phosphoric acid, triethyl ester & 16.41 & 182 & & & & & & & & & & & & & \\
\hline 82 & C4-benzene & 16.43 & 134 & & 0.12 & & & & & & & & & & & \\
\hline 83 & trans methyl decalin & 16.50 & 152 & & & & & & & & & & & & & \\
\hline B4) & C4-benzene & 16.55 & 134 & & & & & & & & & & & & & \\
\hline 85 & cis methyl decalin & 16.74 & 152 & & & & & & & & & & & & & \\
\hline 86. & C5-benzene & 16.79 & 148 & & & & & & & & & & & & & \\
\hline 87 & methyl indan & 17.14 & 132 & & 0.33 & & & & & & & & & & & \\
\hline 88 & 0,0 -diethyl-S-ethyl phosphorothioate & 17.16 & 198 & & & & & & & & & & & & & \\
\hline 89 & methyl indan & 17.29 & 132 & & & & & & & & & & & & & \\
\hline 90 & trichlorobenzene & 17.64 & 180 & & & & & & & & & & & & & \\
\hline 91 & 1-dodecene & 17.66 & 168 & & & & & & & & & & & & & \\
\hline 92 & 1-(4-methylpheny) ethanone & 17.67 & 134 & & & & & & & & & & & & & \\
\hline 93 & N,N-dibutyl-1-butanamine & 17.72 & 142 & & & & & & & & & & & & & \\
\hline 94 & n-dodecane & 17.80 & 170 & & 0.37 & & & & & & & & & & & \\
\hline 95 & naphthalene & 17.79 & 128 & 0.07 & 1.77 & 0.09 & 0.06 & 0.01 & 0.04 & 0.05 & 0.05 & 0.16 & 0.06 & 0.03 & 0.24 & 0.25 \\
\hline 96 & C5-benzene & 17.87 & 148 & & & & & & & & & & & & & \\
\hline 97 & decanal & 17.90 & 156 & & & 0.03 & 0.04 & 0.03 & 0.04 & 0.11 & 0.06 & & 0.07 & 0.02 & & \\
\hline 98 & benzothiophene & 17.90 & 134 & & & & & & & & & & & & & \\
\hline 99 & 1,4-oxathiane, 4,4-dioxide & 18.35 & 136 & & & & & & & & & & & & & \\
\hline 100 & benzothiazole & 18.40 & 135 & & & & & & & & & & & & & \\
\hline 101 & benzene propanenitrile & 18.53 & 131 & & & & & & & & & & & & & \\
\hline 102 & dimethyl indan. & 18.80 & 146 & & & & & & & & & & & & & \\
\hline 103 & 3-tetradecene & 18.85 & 196 & & & & & & & & & & & & & \\
\hline 104 & $\mathrm{~N}$-butyl-N-nitroso N-butanamine & 18.91 & 158 & & & & & & & & & & & & & \\
\hline 105 & 1-tridecene & 19.26 & 182 & & & & & & & & & & & & & \\
\hline 106 & n-tridecane & 19.38 & 184 & & 0.14 & & & & & & & & & & & \\
\hline 107 & N,N-dibutyl formamide & 19.51 & 157 & & & & & & & & & & & & & \\
\hline 108 & 2-methy naphthalene & 19.61 & 142 & 0.04 & 0.21 & 0.04 & 0.03 & & 0.02 & 0.03 & 0.02 & 0.06 & 0.04 & 0.02 & 0.10 & 0.12 \\
\hline 109 & phthalate & 19.80 & 390 & & & & & & & & & & & & & \\
\hline 110 & 1,3-isobenzofurandione & 19.81 & 148 & & & & & & & & & & & & & \\
\hline 111 & 1-methyl naphthalene & 19.91 & 142 & & 0.11 & & & & & & & & & & & \\
\hline 112 & 1-methy-4-(propylthio) benzene & 20.37 & 166 & & & & & & & & & & & & & \\
\hline 113 & 1-tetradecene & 20.76 & 196 & & & & & & & & & & & & & \\
\hline 114 & n-tetradecane & 20.86 & 198 & & 0.08 & & & & & & & & & & & 0.05 \\
\hline 115 & biphenyl & 20.87 & 154 & 0.05 & 0.11 & 0.03 & 0.02 & & 0.06 & 0.02 & & 0.03 & 0.04 & 0.06 & 0.04 & \\
\hline 116 & $1,1^{4}$-oxy bis(benzene) & 21.17 & 186 & & 0.17 & & & & & & & & & & & \\
\hline 117 & 2,4,6-trichloroaniline & 21.20 & 195 & 0.02 & 0.11 & 0.03 & 0.01 & & & & & 0.02 & & & & 0.04 \\
\hline 118 & C2-naphthalene & 21.28 & 156 & & 0.14 & & & & & & & & & & & \\
\hline 119 & C2-naphthalene & 21.51 & 156 & & & 0.03 & 0.02 & & & 0.15 & 0.26 & & 0.11 & 0.10 & & 0.09 \\
\hline 120 & C2-naphthalene & 21.56 & 156 & & & & & & & & & & & & & \\
\hline 121 & tribromobenzene & 21.62 & 312 & & & & & & & & & & & & & \\
\hline 122 & 6,10-dimethyi-5,9-undecadien-2-one & 21.65 & 194 & & & & & & & & & & & & & \\
\hline 123 & 2,6-di-t-butyl-2,5-cyclohexadiene-1,4-dione & 22.06 & 220 & & & & & & & & & & & & & \\
\hline 124 & 1-pentadecene & 22.16 & 210 & & & & & & & & & & & & & \\
\hline 125 & n-pentadecane & 22.26 & 212 & & 0.06 & & & & & & & & & & & \\
\hline 126 & methyl biphenyl & 22.30 & 168 & & & & & & & & & & & & & \\
\hline 127 & 1,2-dihydroacenaphthylene & 22.50 & 168 & & & & & & & & & & & & & \\
\hline
\end{tabular}


Air Monitoring Results

Sample number and room number at the top of each data column

\begin{tabular}{|c|c|c|c|c|c|c|c|c|c|c|c|c|c|c|c|c|}
\hline & Comoound & & & \begin{tabular}{|c|}
37 \\
$E 207$ \\
\end{tabular} & \begin{tabular}{|c|}
163 \\
$E 207$ \\
\end{tabular} & \begin{tabular}{|c|}
35 \\
$E 208$ \\
\end{tabular} & \begin{tabular}{|c|}
34 \\
$E 208$ \\
\end{tabular} & \begin{tabular}{|c|}
38 \\
$E 209$ \\
\end{tabular} & \begin{tabular}{c|}
39 \\
$E 211$
\end{tabular} & \begin{tabular}{|c|}
42 \\
$E 213$ \\
\end{tabular} & \begin{tabular}{|c|}
43 \\
$E 214$ \\
\end{tabular} & \begin{tabular}{|c|}
44 \\
$E 215$ \\
\end{tabular} & \begin{tabular}{|c|}
41 \\
$E 216$ \\
\end{tabular} & \begin{tabular}{|c|}
40 \\
$E 216$ \\
\end{tabular} & \begin{tabular}{|c|}
45 \\
$E 217$ \\
\end{tabular} & $\frac{47}{E 220}$ \\
\hline & $\begin{array}{c}\text { Compound } \\
\text { Tentative Identification }\end{array}$ & RT & MW & $\frac{E 207}{\mathrm{~m}}$ & $\frac{E 207}{m}$ & $\frac{-208}{n}$ & $\frac{208}{s}$ & E209 & c211] & \begin{tabular}{|c|c|}
$E \mathrm{~m}$ \\
$\mathrm{~m}$
\end{tabular} & $\frac{E 214}{m}$ & $\frac{E 215}{m}$ & $E 216$ & E216 & $\frac{E 217}{m}$ & \\
\hline 128 & C3-naphthalene & 22.65 & 170 & & & & & & & & & & & & & \\
\hline 129 & phenyl maleic anhyodride & 22.90 & 214 & & & & & & & & & & & & & \\
\hline 130 & dibenzofuran & 22.93 & 168 & & & & & & & & & & & & & \\
\hline 131 & 1-hexadecene & 22.47 & 224 & & & & & & & & & & & & & \\
\hline 132 & n-hexadecane & 23.57 & 226 & & 0.03 & & & & & & & & & & & \\
\hline 133 & benzenedicarboxylic acid, diethyl ester & 23.64 & 222 & & & & & & & & & & & & & \\
\hline 134 & 1,3-dibromo-2,2-bis(bromomethyl) propane & 24.17 & 384 & & & & & & & & & & & & & \\
\hline 135 & diphenyl diazene & 24.23 & 182 & & & & & & & & & & & & & \\
\hline 136 & benzophenone & 24.30 & 182 & & & & & & & & & & & & & \\
\hline 137 & 1-heptadecene & 24.71 & 238 & & & & & & & & & & & & & \\
\hline 138 & n-heptadecane & 24.81 & 240 & & & & & & & & & & & & & \\
\hline 139 & $\mathrm{~N}$-(phenyimethyiene) benzamine & 24.83 & 181 & & & & & & & & & & & & & \\
\hline 140 & $9 \mathrm{H}$-fiuoren-9-one & 25.28 & 180 & & & & & & & & & & & & & \\
\hline 141 & tribromobenzamine & 25.39 & 327 & & & & & & & & & & & & & \\
\hline 142 & trimethy indan & 25.45 & 160 & & & & & & & & & & & & & \\
\hline 143 & dibenzothiophene & 25.58 & 184 & & & & & & & & & & & & & \\
\hline 144 & tetrabromobenzene & 25.86 & 390 & & & & & & & & & & & & & \\
\hline 145 & n-octadecane & 25.88 & 254 & & & & & & & & & & & & & \\
\hline 146 & phenanthrene/anthracene & 26.37 & 178 & 0.12 & 0.15 & 0.01 & 0.01 & & 0.01 & 0.06 & 0.02 & 0.04 & 0.02 & & 0.02 & 0.13 \\
\hline 147. & methyl dibenzothiophene & 26.98 & 198 & & & & & & & & & & & & & \\
\hline 148 & n-nonadecane & 27.04 & 268 & & & & & & & & & & & & & \\
\hline 149 & 2-methyl anthracene/phenanthrene & 27.48 & 192 & & & & & & & & & & & & & \\
\hline 150 & 1-methyl anthracene/phenanthrene & 27.57 & 192 & & & & & & & & & & & & & \\
\hline 151 & 2,6-dibutyl-2,5-cyclohexadien-1,4-dione & 31.30 & 220 & & 0.19 & & & & & & & & & & & \\
\hline 152 & Total PCB's & & & 8.10 & 1.60 & 0.20 & 0.20 & & & 0.30 & 0.20 & 1.00 & & & 0.40 & 11.90 \\
\hline & & & & & & & & & & & & & & & & \\
\hline & & & & & & & & & & & & & & & & \\
\hline & & & & & & & & & & & & & & & & \\
\hline & & & & & & & & & & & & & & & & \\
\hline & & & & & & & & & & & & & & & & \\
\hline & & & & & & & & & & & & & & & & \\
\hline & & & & & & & & & & & & & & & & \\
\hline & & & & & & & & & & & & & & & & \\
\hline & & & & & & & & & & & & & & & & \\
\hline & & & & & & & & & & & & & & & & \\
\hline & & & & & & & & & & & & & & & & \\
\hline & & & & & & & & & & & & & & & & \\
\hline & & & & & & & & & & & & & & & & \\
\hline & & & & & & & & & & & & & & & & \\
\hline & & & & & & & & & & & & & & & & \\
\hline & & & & & & & & & & & & & & & & \\
\hline & & & & & & & & & & & & & & & & \\
\hline & & & & & & & & & & & & & & & & \\
\hline & & & & & & & & & & & & & & & & \\
\hline & & & & & & & & & & & & & & & & \\
\hline & & & & & & & & & & & & & & & & \\
\hline & & & & & & & & & & & & & & & & \\
\hline & & & & & & & & & & & & & & & & \\
\hline & & & & & & & & & & & & & & & & \\
\hline & & & & & & & & & & & & & & & & \\
\hline & & & & & & & & & & & & & & & & \\
\hline & & & & & & & & & & & & & & & & \\
\hline & & & & & & & & & & & & & & & & \\
\hline & & & & & & & & & & & & & & & & \\
\hline & & & & & & & & & & & & & & & & \\
\hline & & & & & & & & & & & & & & & & \\
\hline & & & & & & & & & & & & & & & & \\
\hline & & & & & & & & & & & & & & & & \\
\hline & & & & & & & & & & & & & & & & \\
\hline & & & & & & & & & & & & & & & & \\
\hline & & & & & & & & & & & & & & & & \\
\hline & & & & & & & & & & & & & & & & \\
\hline & 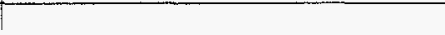 & . & | & 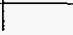 & . & 1 & | & & 列 & & & & & & & \\
\hline
\end{tabular}




\begin{tabular}{|c|c|c|c|c|c|c|c|c|c|c|c|c|c|c|c|c|}
\hline & & & & 48 & 49 & 50 & 51 & 137 & 156 & 157 & 158 & 159 & 160 & 161 & 162 & 52 \\
\hline & Compound & & & $E 220$ & E220 & $E 220$ & & E220 & $E 220$ & E220 & E220 & E220 & & & & E301 \\
\hline & Tentative Identification & RT & MW & sw & $w$ & $\mathrm{nw}$ & 0 & se & se & ne & $n w$ & nw & 0 & 0 & 0 & $\mathrm{~m}$ \\
\hline 1 & sulfur dioxide & 1.55 & 64 & & & & & & & & & & & 0.96 & & \\
\hline 2 & 2-propanone & 1.89 & 58 & & & & & & & & & & & & & \\
\hline 3 & 1-fluoro-1,1-dichloroethane & 1.95 & 116 & & & & & & & & & & & & & \\
\hline 4 & N-ethyd ethanamine & 2.36 & 73 & & & & & & & & & & & & & \\
\hline 5 & 2-butanone & 2.56 & 72 & & & & & & & & & & & & & \\
\hline 6 & $\mathrm{~N}$-ethyl- $\mathrm{N}$-methyl ethanamine & 2.94 & 87 & & & & & & & & & & & & & \\
\hline 7 & dichloromethane & 3.15 & 84 & & & & & & & & & & & & & \\
\hline 8 & $\mathrm{~N}$-(1-methylethy)-2-propanamine & 3.59 & 101 & & & & & & & & & & & & & \\
\hline 9 & benzene & 3.64 & 78 & & 0.06 & & & & 1.77 & 0.24 & 3.17 & 2.72 & 0.83 & 0.40 & 0.53 & \\
\hline 10 & 2-pentanone & 4.08 & 86 & & & & & & & & & & & & & \\
\hline 11 & trichtoroethene & 4.47 & 130 & & & & & & 1 & 0.02 & 0.13 & 0.15 & 0.02 & 0.02 & $i$ & \\
\hline 12 & N-(1-methylethylidene)-2-propanamine & 4.62 & 99 & & & & & & & & & & & & & \\
\hline 13 & toluene & 6.97 & 92 & 0.54 & 0.74 & 0.59 & 0.00 & 0.02 & 0.97 & 0.21 & 1.24 & 1.28 & 1.48 & 1.06 & 0.51 & $\underline{0.03}$ \\
\hline 14 & $\mathrm{~N}$-methyl-N-(1-methylethyl)-2-propanamine & 7.27 & 115 & & & & & & & & & & & & & \\
\hline 15 & 3-hexanone & 7.75 & 100 & & & & & & & & & & & & & \\
\hline 16 & 2-hexanone & 7.92 & 100 & & & & & & & & & & & & & \\
\hline 17 & n-octane & 8.29 & 114 & & & & & & & & & & & & & \\
\hline 18 & hexanal & 8.33 & 100 & & & & & & 0.49 & 0.28 & 0.57 & 0.37 & & 0.59 & & \\
\hline 19 & tetrachloroethene & 8.79 & 164 & 0.03 & 0.04 & & & & & & & & & & & 0.00 \\
\hline 20 & methyl ethyl disulfide & 9.68 & 108 & & & & & & & & & & & & & \\
\hline 21 & chlorobenzene & 10.05 & 112 & & & & & & & & & & & & & \\
\hline 22 & ethyl benzene & 10.57 & 106 & 0.11 & 0.13 & 0.12 & & & 0.21 & 0.12 & 0.23 & 0.25 & 0.21 & 0.14 & 0.03 & 0.01 \\
\hline 23 & p,m-xylene & 10.81 & 106 & 0.41 & 0.44 & 0.38 & & & 0.52 & 0.16 & 0.44 & 0.53 & 0.53 & 0.25 & 0.10 & 0.01 \\
\hline 24 & 1,4-oxathiane & 11.00 & 104 & & & & & & & & & & & & & \\
\hline 25 & styrene & 11.30 & 118 & & 0.06 & 0.03 & & & & & & & & & 0.06 & \\
\hline 26 & 3-heptanone & 11.37 & 114 & & & & & & & & & & & & & \\
\hline 27 & o-xylene & 11.49 & 106 & 0.14 & 0.17 & 0.15 & & & 0.29 & 0.1 & 0.18 & 0.29 & 0.28 & 0.10 & 0.05 & 0.01 \\
\hline 28 & heptanal & 11.60 & 114 & & & & & & & & 0.94 & 0.41 & & & & \\
\hline 29 & n-nonane & 11.67 & 128 & 0.03 & 0.03 & 0.03 & & & 0.31 & 0.33 & & & & & & 0.00 \\
\hline 30 & C3-benzene & 12.20 & 120 & & & & & & & & & & & & & \\
\hline 31 & N-butylidene-1-butanamine & 12.28 & 127 & & & & & & & & & & & & & \\
\hline 32 & C3-benzene & 12.33 & 120 & & & & & & & & & & & & & \\
\hline 33 & 3-methyl nonane & 12.51 & 142 & & & & & & 0.18 & & & 0.30 & & & & \\
\hline 34 & pinene & 12.61 & 136 & 0.05 & 0.05 & 0.02 & & & & & & 0.33 & & & 0.14 & \\
\hline 35 & 2-methyl cyclohexanol & 12.62 & 114 & & & & & & & & & & & & & \\
\hline 36 & 3-octanone & 12.71 & 128 & & & & & & & & & & & & & \\
\hline 37 & 2-ethyl hexanal 12.72 & 13.02 & 128 & & & & & & & & & & & & & \\
\hline 38 & 1-chloro-2-methyl benzene & 13.03 & 126 & & & & & & & & & & & & & \\
\hline 39 & propyl benzene & 13.05 & 120 & & 0.07 & 0.06 & & & 0.32 & & & & & & & \\
\hline 40 & N-butyl-1-butanamine & 13.17 & 129 & & & & & & & & & & & & & \\
\hline 41 & benzaldehyde & 13.18 & 106 & 0.08 & 0.07 & 0.09 & & & 2.03 & 0.93 & 2.77 & 2.08 & 5.25 & 6.34 & 1.43 & 0.04 \\
\hline 42 & C3-benzene & 13.25 & 120 & 0.16 & 0.19 & 0.20 & & & 0.25 & & & & & & & \\
\hline 43 & C3-benzene & 13.41 & 120 & 0.06 & 0.07 & 0.05 & & & & & & 0.21 & & & & \\
\hline 44 & phenol & 13.57 & 94 & & & & & & & & & & 1.55 & & & \\
\hline 45 & Taniline & 13.61 & 93 & & & & & & & & & & & & & \\
\hline 46 & C3-benzene & 13.68 & 120 & & & & & & & & & & & & & \\
\hline 47 & 6-methyl-5-hepten-2-one & 13.69 & 126 & & & & & & 0.15 & 0.17 & 0.51 & & & 0.21 & & \\
\hline 48 & benzonitrile & 13.72 & 103 & & & & & & 0.42 & & 0.58 & 0.46 & 0.41 & 0.32 & 0.14 & \\
\hline 49 & 1-decene & 13.83 & 140 & & & & & & & & & & & & & \\
\hline 50 & C3-benzene & 13.99 & 120 & & & & & & & & & & 0.23 & & & \\
\hline 51 & n-decane & 14.07 & 142 & 0.09 & 0.09 & 0.10 & & & 0.19 & & & 0.26 & & & & 0.01 \\
\hline 52 & octanal & 14.09 & 128 & & & & & & 0.29 & 0.38 & 1.22 & & 0.15 & 0.35 & 0.03 & \\
\hline 53 & dichlorobenzene & 14.40 & 146 & & 0.03 & 0.03 & & & & & & & & & & \\
\hline 54 & 4-methyl decane & 14.56 & 156 & & & & & & & & & & & & & \\
\hline 55 & C3-benzene & 14.57 & 120 & & & & & & 0.27 & & 0.72 & 0.49 & & & & \\
\hline 56 & C4-benzene & 14.60 & 134 & & & & & & 0.13 & 0.18 & 0.51 & 0.15 & & & & \\
\hline 57 & 2-ethyl hexanol & 14.64 & 130 & 0.16 & 0.21 & & & & 0.57 & 0.27 & 0.72 & 0.39 & & & 0.26 & \\
\hline 58 & limonene & 14.75 & 136 & 0.04 & 0.05 & 0.05 & & & & & & & & & & \\
\hline 59 & benzene methanol & 14.77 & 108 & & & & & & & & & & & & & \\
\hline 60 & indan & 14.87 & 118 & & & & & & 0.17 & & & 0.25 & & & & \\
\hline 61 & dichlorobenzene & 14.89 & 146 & & & & & & & & & & & & & \\
\hline 62 & $\mathrm{~N}$-ethyl-N-(1-methylethyl)-2-propanamine & 15.07 & 129 & & & & & & & & & & & & & \\
\hline 63 & C4-benzene & 15.10 & 134 & & & & & & 0.27 & 0.23 & & 0.34 & & & & \\
\hline
\end{tabular}




\begin{tabular}{|c|c|c|c|c|c|c|c|c|c|c|c|c|c|c|c|c|}
\hline & & & & 48 & 49 & 50 & 51 & 137 & 156 & 157 & 158 & 159 & 160 & 161 & 162 & 52 \\
\hline & Compound & & & E220 & E220 & E220 & & $E 220$ & E220 & E220 & $E 220$ & E220 & & & & E301 \\
\hline & Tentative Identification & RT & MW & sw & $w$ & $n w$ & 0 & se & se & ne & nw & nw & 0 & 0 & 0 & $\mathrm{~m}$ \\
\hline 64 & 5-methyl decane & 15.21 & \begin{tabular}{|l|l|}
156 \\
\end{tabular} & & & & & & & & & & & & & \\
\hline 65 & 4-methyl decane & 15.29 & 956 & & & & & & & & & & & & & \\
\hline 66 & C4-benzene & 15.30 & 134 & & & & & & 0.32 & & 0.4 & 0.41 & & & & \\
\hline 67 & 2-methyl decane & 15.34 & 156 & & & & & & & & & & & & & \\
\hline 68 & 1,4-dithiane & 15.39 & 120 & & & & & & & & & & & & & \\
\hline 69 & acelophenone & 15.47 & 120 & & & & 0.00 & 0.01 & 1.06 & 0.68 & 1.57 & 1.53 & 3.84 & 3.41 & 1.34 & 0.03 \\
\hline 70 & C4-benzene & 15.47 & 134 & & & & & & 0.08 & & & & & & & \\
\hline 71 & 3-methyl decane & 15.48 & 156 & & & & & & & & & & & & & \\
\hline 72 & methyl benzaldehyde & 15.54 & 120 & & & & & & & & & & & & & \\
\hline 73 & C4-benzene & 15.64 & 134 & & & & & & & & & 0.25 & & & & \\
\hline 74 & C4-benzene & 15.72 & 134 & & & & & & & & & 0.24 & & & & \\
\hline 75 & A,A-dimethyl benzene methanol & 15.80 & 136 & & & & & & & & & & & & & \\
\hline 76 & C4-benzene & 15.86 & 134 & & & & & & & & & & & & & \\
\hline 77 & 1-undecene & 15.95 & 154 & & & & & & & & & & & & & \\
\hline 78 & n-undecane & 16.05 & 156 & 0.07 & 0.07 & 0.07 & & & 0.25 & & & 0.68 & & 0.10 & & 0.01 \\
\hline 79 & nonanal & 16.12 & 142 & & & 0.04 & 0.01 & & 0.86 & 0.76 & 2.53 & 0.83 & 0.44 & 1.27 & 0.10 & 0.01 \\
\hline 80 & C4-benzene & 16.24 & 134 & & & & & & & & & & & & & \\
\hline 81 & phosphoric acid, triethyl ester & 16.41 & 182 & & & & & & & & & & & & & \\
\hline 82 & C4-benzene & 16.43 & 134 & & & & & & & & & & & & & \\
\hline 83 & trans methy decalin & 16.50 & 152 & & & & & & & & & & & & & \\
\hline 84 & C4-benzene & 16.55 & 134 & & & & & & & & & & & & & \\
\hline 85 & cis methyl decalin & 16.74 & 152 & & & & & & & & & & & & & \\
\hline 86 & C5-benzene & 16.79 & 148 & & & & & & & & & & & & & \\
\hline 87 & methyl indan & 17.14 & 132 & & & & E & & & & & & & & & \\
\hline 88 & 0,O-diethyl-S-ethyl phosphorothioate & 17.16 & 198 & & & & & & & & & & & & & \\
\hline 89 & methyl indan & 17.29 & 132 & & & & & & 0.13 & & & 0.26 & & & & \\
\hline 90 & trichlorobenzene & 17.64 & 180 & & & & & & & 0.15 & & & & & & \\
\hline 91 & 1-dodecene & 17.66 & 168 & & & & & & & & & & & & & \\
\hline 92 & 1-(4-methyiphenyl) ethanone & 17.67 & 134 & & & & & & & & & & & & & \\
\hline 93 & $N, N$-dibutyl-1-butanamine & 17.72 & 142 & & & & & & & & & & & & & \\
\hline 94 & n-dodecane & 17.80 & 170 & & & & & & 0.09 & & & 0.18 & & & & \\
\hline 95 & naphthalene & 17.79 & 128 & 0.19 & 0.25 & 0.23 & & 0.01 & 0.44 & 0.37 & 0.83 & 0.68 & & & & 0.01 \\
\hline 96 & C5-benzene & 17.87 & 148 & & & & & & & & & 0.20 & & & & \\
\hline 97 & decanal & 17.90 & 156 & & & & 0.00 & & 0.68 & 0.7 & 1.38 & 0.37 & 0.38 & 1.36 & 0.08 & 0.01 \\
\hline 98 & benzothiophene & 17.90 & 134 & & & & & & & & & & & & & \\
\hline 99 & 1,4-oxathiane, 4,4-dioxide & 18.35 & 136 & & & & & & & & & & & & & \\
\hline 100 & benzothiazole & 18.40 & 135 & & & & 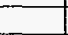 & & & & 0.07 & & & & & \\
\hline 101 & benzene propanenitrile & 18.53 & 131 & & & & & & & & & & & & & \\
\hline 102 & dimethyl indan & 18.80 & 146 & & & & & & & & & & & & & \\
\hline 103 & 3-tetradecene & 48.85 & 196 & & & & & & & & & & & & & \\
\hline 104 & $\mathrm{~N}$-butyl-N-nitroso $\mathrm{N}$-butanamine & 18.91 & 158 & & & & & & & & & & & & & \\
\hline 105 & 1-tridecene & 19.26 & 182 & & & & & & & & & & & & & \\
\hline 106 & n-tridecane & 19.38 & 184 & & & & & & 0.17 & & & 0.32 & & & & \\
\hline 107 & $\mathrm{~N}, \mathrm{~N}$-dibutyl formamide & 19.51 & 157 & & & & & & & & & & & & & \\
\hline 108 & 2 -methyt naphthalene & 19.61 & 142 & 0.08 & 0.13 & 0.11 & & & & & & 0.26 & & & & \\
\hline 909 & phthalate & 19.80 & 390 & & & & & & & & & & & & & \\
\hline 910 & 1,3-isobenzofurandione & 19.81 & 148 & & & & & & & & & 0.13 & & & 0.06 & \\
\hline 111 & 1-meihyl naphthalene & 19.91 & 142 & & & & & & & & & 0.16 & & & & \\
\hline 912 & 1-methy-4-(propylthio) benzene & 20.37 & 166 & & & & & & & & & & & & & \\
\hline 113 & 1-tetradecene & 20.76 & 196 & & & & 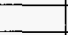 & & & & 0.21 & & & & & \\
\hline 114 & n-tetradecane & 20.86 & 198 & 0.05 & 0.05 & & & & 0.63 & & & 0.15 & & & & \\
\hline 115 & biphenyt & 20.87 & 154 & & & 0.06 & & & & & & 0.08 & & & & 0.00 \\
\hline 116 & 1,1'-oxy bis(benzene) & 21.17 & 186 & & & & & & & & & 0.07 & & & & \\
\hline 117 & 2,4,6-trichloroaniline & 21.20 & 195 & 0.02 & 0.04 & 0.04 & & & 0.17 & & 0.14 & 0.17 & & & & \\
\hline 118 & C2-naphthalene & 21.28 & 156 & & & & & & 0.14 & & & 0.08 & & & & \\
\hline 119 & C2-naphthalene & 21.51 & 156 & 0.03 & 0.05 & 0.04 & & & & & 0.15 & 0.09 & & & & \\
\hline 120 & C2-naphthalene & 21.56 & 156 & & & & & & & & 0.2 & & & & & \\
\hline 121 & tribromobenzene & 21.62 & 312 & & & & & & & & & & & & & \\
\hline 122 & 6,10-dimethyl-5,9-undecadien-2-one & 21.65 & 194 & & & & & & & & & & & & & \\
\hline 123 & 2,6-di-t-buty-2,5-cyclohexadiene-1,4-dione & 22.06 & 220 & & & & & & & & & & & & & \\
\hline 124 & 1-pentadecene & 22.16 & 210 & & & & & & & & & & & & & \\
\hline 125 & n-pentadecane & 22.26 & 212 & & & & & & 0.09 & & 0.1 & 0.11 & & & & \\
\hline 126 & methyl biphenyl & 22.30 & 168 & & & & & & & & & & & & & \\
\hline 127 & 1,2-dihydroacenaphthylene & $22.50 \mid$ & 168 & & & & & & 0.06 & & 0.08 & 0.06 & & & & \\
\hline
\end{tabular}


Air Monitoring Results

Sample number and room number at the top of each data column

\begin{tabular}{|c|c|c|c|c|c|c|c|c|c|c|c|c|c|c|c|c|}
\hline & & & & 48 & 49 & 50 & 51 & 137 & 156 & 157 & 158 & 159 & 160 & 161 & 162 & 52 \\
\hline & Compound & & & $E 220$ & E220 & $E 220$ & & $E 220$ & E220 & $E 220$ & E220 & $E 220$ & & & & $E 301$ \\
\hline & Tentative Identification & RT & MW & sw & $w$ & nw & 0 & se & se & ne & nw & nw & 0 & 0 & o. & $\mathrm{m}$ \\
\hline 128 & C3-naphthalene & 22.65 & 170 & & & & & & & & & & & & & \\
\hline 129 & phenyl maleic anhydride & 22.90 & 214 & & & $\div$ & & & 0.15 & & 0.14 & & 0.21 & 0.13 & & \\
\hline 130 & dibenzofuran & 22.93 & 168 & & & & & & 0.09 & & 0.11 & 0.09 & & & & \\
\hline 1311 & 1-hexadecene & 22.47 & 224 & & & & & & & & & & & & & \\
\hline 132 & n-hexadecane & 23.57 & 226 & & & & & & 0.11 & & 0.09 & 0.08 & & & & \\
\hline 133 & benzenedicarboxylic acid, diethyl ester & 23.64 & 222 & & & & & & 0.07 & & 0.14 & 0.07 & & & & \\
\hline 134 & 1,3-dibromo-2,2-bis(bromomethyl) propane & 24.17 & 384 & & & & & & & & & & & & & \\
\hline 135 & diphenyl diazene & 24.23 & 182 & & & & & & & & & & & & & \\
\hline 136 & benzophenone & 24.30 & 182 & & & & & & 0.12 & & 0.17 & 0.17 & 0.33 & 0.24 & 0.13 & \\
\hline $137 \mid$ & 1-heptadecene & 24.71 & 238 & & & & & & & & & & & & & \\
\hline 138 & n-heptadecane & 24.81 & 240 & & & & & & 0.22 & & & 0.09 & & & & \\
\hline 139 & $\mathrm{~N}$-(phenyimethylene) benzamine & 24.83 & 181 & & & & & & & & & & & & & \\
\hline 140 & 9H-fluoren-g-one & 25.28 & 180 & & & & & & 0.27 & & 0.13 & & & & & \\
\hline 141 & tribromobenzamine & 25.39 & 327 & & & & & & & & & & & & & \\
\hline 142 & trimethyl indan & 25.45 & 160 & & & & & & & & & & & & & \\
\hline 143 & dibenzothiophene & 25.58 & 184 & & & & & & & & & & & & & \\
\hline 144 & tetrabromobenzene & 25.86 & 390 & & & & & & & & & & & & & \\
\hline 145 & n-octadecane & 25.88 & 254 & & & & & & & & & 0.05 & & & & \\
\hline 146 & phenanthrene/anthracene & 26.37 & 178 & & 0.03 & 0.05 & 0.04 & 0.00 & 0.28 & & 0.31 & 0.30 & & & & 0.01 \\
\hline 147 & methyl dibenzothiophene & 26.98 & 198 & & & & & & & & & & & & & \\
\hline 148 & n-nonadecane & 27.04 & 268 & & & & & & & & & & & & & \\
\hline 149 & 2-methyl anthracene/phenanthrene & 27.48 & 192 & & & & & & & & & & & & & \\
\hline 150 & 1-methy anthracene/phenanthrene & 27.57 & 192 & & & & & & & & & & & & & \\
\hline 151 & 2,6-dibutyi-2,5-cyclohexadien-1,4-dione & 31.30 & 220 & & & & & & & & & & & 0.54 & & \\
\hline 152 & Total PCB's & & & 1.80 & & 1.10 & & 1.00 & & 0.4 & & & & & & 0.20 \\
\hline & & & & & & & & & & & & & & & & \\
\hline & & & & & & & & & & & & & & & & \\
\hline & & & & & & & & & & & & & & & & \\
\hline & & & & & & & & & & & & & & & & \\
\hline & & & & & & & & & & & & & & & & \\
\hline & & & & & & & & & & & & & & & & \\
\hline & & & & & & & & & & & & & & & & \\
\hline & & & & & & & & & & & & & & & & \\
\hline & & & & & & & & & & & & & & & & \\
\hline & & & & & & & & & & & & & & & & \\
\hline & & & & & & & & & & & & & & & & \\
\hline & & & & & & & & & & & & & & & & \\
\hline & & & & & & & & & & & & & & & & \\
\hline & & & & & & & & & & & & & & & & \\
\hline & & & & & & & & & & & & & & & & \\
\hline & & & & & & & & & & & & & & & & \\
\hline & & & & & & & & & & & & & & & & \\
\hline & & & & & & & & & & & & & & & & \\
\hline & & & & & & & & & & & & & & & & \\
\hline & & & & & & & & & & & & & & & & \\
\hline & & & & & & & & & & & & & & & & \\
\hline & & & & & & & & & & & & & & & & \\
\hline & & & & & & & & & & & & & & & & \\
\hline & & & & & & & & & & & & & & & & \\
\hline & & & & & & & & & & & & & & & & \\
\hline & & & & & & & & & & & & & & & & \\
\hline & & & & & & & & & & & & & & & & \\
\hline & & & & & & & & & & & & & & & & \\
\hline & & & & & & & & & & & & & & & & \\
\hline & & & & & & & & & & & & & & & & \\
\hline & & & & & & & & & & & & & & & & \\
\hline & & & & & & & & & & & & & & & & \\
\hline & & & & & & & & & & & & & & & & \\
\hline & & & & & & & & & & & & & & & & \\
\hline & & & & & & & & & & & & & & & & \\
\hline & & & & & & & & & & & & & & & & \\
\hline & & & & & & & & & & & & & & & & \\
\hline & . & 1 & 1 & 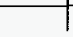 & 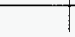 & 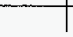 & 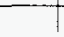 & 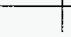 & . & 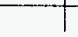 & & & & & & \\
\hline
\end{tabular}




\begin{tabular}{|c|c|c|c|c|c|c|c|c|c|c|c|c|c|c|c|c|}
\hline & & & & 56 & 57 & 54 & 164 & 55 & 53 & 60 & 61 & 134 & 58 & 59 & 63 & 64 \\
\hline & Compound & & & $E 303$ & $E 303$ & $E 305$ & $E 305$ & E305 & E306 & E308 & E308 & $E 308$ & E309 & E309 & E310 & E311 \\
\hline & Tentative Identification & RT & MW & $n$ & $s$ & $s$ & ne & $n$ & $\mathrm{~m}$ & s & $n$ & & $n$ & $s$ & $\mathrm{~m}$ & $m$ \\
\hline 1 & sulfur dioxide & 1.55 & 64 & & & & & & & & & & & & & \\
\hline 2 & 2-propanone & 1.89 & 58 & & & & & & & & & & & & & \\
\hline 3 & 1-fluoro-1,1-dichloroethane & 1.95 & 116 & & & & & & & & & & & & & \\
\hline 4 & N-ethyl ethanamine & 2.36 & 73 & & & & & & & & & & & & & \\
\hline 5 & 2-butanone & 2.56 & 72 & & & & & & & & & & & & & \\
\hline 6 & $\mathrm{~N}$-ethyl-N-methyl ethanamine & 2.94 & 87 & & & & & & & & & & & & & \\
\hline 7 & dichloromethane & 3.15 & 84 & & & & & 0.11 & & & & & & & & \\
\hline 8 & N-(1-methylethyl)-2-propanamine & 3.59 & 101 & & & & & & & & & & & & & \\
\hline 9 & benzene & 3.64 & 78 & & & 0.01 & 0.40 & 0.04 & & 0.03 & 0.06 & & & 0.03 & 0.05 & 0.06 \\
\hline 10 & 2-pentanone & 4.08 & 86 & & & & & & & & & & & & & \\
\hline 11 & trichloroethene & 4.47 & 130 & & & & 0.14 & & & & & & & & 0.02 & \\
\hline 12 & $N-(1-m e t h y j e t h y$ tidene)-2-propanamine & 4.62 & 99 & & & & & & & & & & & & & \\
\hline 13 & toluene & 6.97 & 92 & 0.07 & 0.05 & 0.07 & 0.90 & 0.16 & 0.11 & 0.74 & 0.56 & & 0.24 & 0.17 & 0.51 & 0.68 \\
\hline 14 & N-methyl-N-(1-methylethyl)-2-propanamine & 7.27 & 115 & & & & & & & & & & & & & \\
\hline 15 & 3-hexanone & 7.75 & 100 & & & & & & & & & & & & & \\
\hline 16 & 2-hexanone & 7.92 & 100 & & & & & & & & & & & & & \\
\hline 17 & n-octane & 8.29 & 114 & & & & 0.07 & & & & & & & & & \\
\hline 18 & hexanal & 8.33 & 100 & & & & & & & & & & & & & \\
\hline 19 & tetrachloroethene & 8.79 & 164 & 0.02 & 0.02 & 0.01 & 0.11 & 0.02 & 0.01 & 0.04 & 0.03 & & & 0.04 & 0.03 & 0.08 \\
\hline 20 & methyl ethyl disulfide & 9.68 & 108 & & & & & & & & & & & & & \\
\hline 21 & chlorobenzene & 10.05 & 112 & & & & & & & & & & & & & \\
\hline 22 & ethyl benzene & 10.57 & 106 & 0.02 & 0.01 & 0.02 & 0.18 & 0.03 & 0.02 & 0.18 & 0.14 & & 0.05 & 0.04 & 0.15 & 0.17 \\
\hline 23 & $\mathrm{p}, \mathrm{m}$-xylene & 10.81 & 106 & 0.03 & 0.02 & 0.04 & 0.49 & 0.06 & 0.05 & 0.60 & 0.48 & & 0.07 & 0.06 & 0.48 & 0.48 \\
\hline 24 & 1,4-oxathiane & 11.00 & 104 & & & & & & & & & & & & & \\
\hline 25 & styrene & 11.30 & 118 & & 0.00 & 0.01 & 0.04 & 0.01 & 0.01 & 0.08 & 0.03 & & & 0.01 & & \\
\hline 26 & 3-heptanone & 11.37 & 114 & & & & & & & & & & & & & 0.08 \\
\hline 27 & o-xylene & 11.49 & 406 & & 0.01 & 0.02 & 0.28 & 0.03 & 0.02 & 0.22 & 0.17 & & & 0.03 & 0.18 & 0.19 \\
\hline 28. & heptanal & 11.60 & 114 & & & & & & & & & & & & & \\
\hline 29 & n-nonane & 11.67 & 128 & 0.03 & \begin{tabular}{l|l|} 
& 0.00 \\
\end{tabular} & 0.01 & & 0.02 & 0.02 & 0.04 & 0.03 & & & 0.02 & 0.03 & 0.04 \\
\hline 30 & C3-benzene & 12.20 & 120 & & & & & & & & & & & & & \\
\hline 31 & $\mathrm{~N}$-butylidene-1-butanamine & 12.28 & 127 & & & & & & & & & & & & & \\
\hline 32 & C3-benzene & 12.33 & 120 & & & & & & & & & & & & & \\
\hline 33 & 3-methyl nonane & 12.51 & 142 & & & & & & & & & & & & & \\
\hline 34 & pinene & 12.61 & \begin{tabular}{|l|l|}
136 \\
\end{tabular} & & & & & & & 0.05 & 0.03 & & & & & \\
\hline 35 & 2-methyl cyclohexanol & 12.62 & 114 & & & & & & & & & & & & & \\
\hline 36 & 3-octanone & 12.71 & 128 & & & & & & & & & & & & & \\
\hline 37 & 2-ethyl hexanal & 13.02 & 128 & & & & & & & 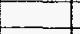 & & & & & & \\
\hline 38 & 1-chloro-2-methyl benzene & 13.03 & 126 & & & & & & & & & & & & & \\
\hline 39 & propyl benzene & 13.05 & 120 & 0.01 & & & 0.10 & & 0.01 & 0.06 & 0.05 & & & 0.01 & 0.06 & 0.06 \\
\hline 40 & $\mathrm{~N}$-butyl-1-butanamine & 13.17 & 129 & & & & & & & & & & & & & \\
\hline 41 & benzaldehyde & 13.18 & 106 & 0.08 & 0.06 & 0.05 & 0.48 & 0.08 & 0.05 & 0.05 & 0.04 & & 0.14 & 0.12 & 0.08 & 0.09 \\
\hline 42 & C3-benzene & 13.25 & 120 & & & & 0.26 & & & 0.27 & 0.21 & & & & 0.23 & 0.22 \\
\hline 43 & C3-benzene & 13.41 & 120 & & & & 0.05 & & & 0.09 & 0.07 & & & & 0.07 & 0.06 \\
\hline 44 & phenol & 13.57 & 94 & & & & & & & & & & & 0.08 & & \\
\hline 45 & aniline & 13.61 & 93 & & & & & & & & & & & & & \\
\hline 46 & C3-benzene & 13.68 & 120 & & & & & & & 0.16 & 0.07 & & & & 0.10 & 0.13 \\
\hline 47 & 6-methyl-5-hepten-2-one & 13.69 & 126 & & & & & & & & & & & & & \\
\hline 48 & benzonitrile & 13.72 & 103 & & & & 0.07 & & 0.05 & & & & & & & \\
\hline 49 & 1-decene & 13.83 & 140 & & & & & & & & & & & & & \\
\hline 50 & C3-benzene & 13.99 & 120 & & & & & & & & & & & & 0.39 & 0.33 \\
\hline 51 & n-decane & 14.07 & 142 & & 0.01 & 0.03 & & 0.06 & 0.04 & 0.11 & 0.08 & & & 0.06 & 0.11 & 0.11 \\
\hline 52 & Octanal & 14.09 & 128 & & & & & & & & & & & & & \\
\hline 53 & dichlorobenzene & \begin{tabular}{|c|}
14.40 \\
\end{tabular} & 146 & & & 0.00 & & & & 0.04 & 0.03 & & & 0.02 & & 0.03 \\
\hline 54 & 4-methyl decane & 14.56 & 156 & & & & & & & & & & & 0.01 & & \\
\hline 55 & C3-benzene & 14.57 & 120 & & & & 0.08 & & & & & & & 0.03 & & \\
\hline 56 & C4-benzene & 14.60 & 134 & & & & & & & 0.14 & 0.11 & & & & & $\overline{0.14}$ \\
\hline 57 & 2-ethyl hexanol & 14.64 & 130 & 0.03 & 0.01 & 0.02 & 0.07 & 0.43 & 0.02 & & & & & & 0.12 & \\
\hline 58 & limonene & 14.75 & 136 & & & & 0.09 & & & 0.15 & 0.06 & & & & 0.04 & 0.04 \\
\hline 59 & benzene methanol & 14.77 & 108 & & & & & & & & & & & 0.07 & & \\
\hline 60 & indan & 14.87 & 118 & & & & 0.03 & & & & & & & & & \\
\hline 61 & dichlorobenzene & 14.89 & 146 & & & & & & & & & & & & & \\
\hline 62 & $\mathrm{~N}$-ethyl-N-(1-methylethy)-2-propanamine & 15.07 & 129 & & & & & & & & & & & & & \\
\hline 63 & C4-benzene & $|15.10|$ & 134 & & & & & & & & 0.07 & & & 0.20 & 0.08 & 0.07 \\
\hline
\end{tabular}




\begin{tabular}{|c|c|c|c|c|c|c|c|c|c|c|c|c|c|c|c|c|}
\hline & & & & 56 & 57 & 54 & 164 & 55 & 53 & 60 & 61 & 134 & 58 & 59 & \begin{tabular}{c|}
63 \\
$524 n$
\end{tabular} & $\begin{array}{c}64 \\
E 311\end{array}$ \\
\hline & Compound & & & E303 & $E 303$ & E305 & E305 & E305 & E306 & $E 308$ & E308 & E308 & E309 & E309 & E310 & E311 \\
\hline & Tentative Identification & RT & MW & $n$ & $s$ & $\mathbf{s}$ & ne & $n$ & $\mathrm{~m}$ & $s$ & $n$ & & $n$ & $s$ & $\mathrm{~m}$ & $\mathrm{~m}$ \\
\hline 64 & 5-methyl decane & \begin{tabular}{|l|}
15.21 \\
\end{tabular} & 156 & & & & & & & & & & & 0.02 & 0.05 & 0.05 \\
\hline 65 & 4-methyl decane & \begin{tabular}{|l|}
15.29 \\
\end{tabular} & 156 & & & & & & & & & & & & & \\
\hline 66 & C4-benzene & 15.30 & 134 & & & & & & & & & & & & 0.06 & 0.06 \\
\hline 67 & 2-methyl decane & 15.34 & 156 & & & & & & & & & & & 0.02 & & \\
\hline 68 & 1,4-dithiane & 15.39 & 120 & & & & & & & & & & & & & \\
\hline 69 & acetophenone & \begin{tabular}{|l|}
15.47 \\
\end{tabular} & 120 & 0.08 & 0.06 & 0.04 & 0.19 & 0.07 & 0.03 & & & & 0.09 & 0.11 & & \\
\hline 70 & C4-benzene & 15.47 & 134 & & & & & & & & & & & & 0.08 & 0.10 \\
\hline 71 & 3-methyl decane & 15.48 & 156 & & & & & & & & & & & & & \\
\hline 72 & methyl benzaldehyde & 15.51 & 120 & & & & & & & & & & & & & \\
\hline 73 & C4-benzene & 15.64 & 134 & & & & 0.03 & & & & & & & & & \\
\hline 74 & C4-benzene & \begin{tabular}{|l|}
15.72 \\
\end{tabular} & 134 & & & & & & & & & & & & & \\
\hline 75 & A,A-dimethyl benzene methanol & 15.80 & 136 & & & & & & & & & & & & & \\
\hline 76 & C4-benzene & 15.86 & 134 & & & & & & & 0.08 & 0.06 & & & & 0.07 & 0.08 \\
\hline 77 & 1-undecene & 15.95 & 154 & & & & & & & & & & & & & \\
\hline 78. & n-undecane & 16.05 & 156 & 0.03 & 0.02 & 0.03 & 0.11 & 0.04 & 0.03 & 0.12 & 0.09 & & 0.07 & 0.08 & 0.14 & 0.12 \\
\hline 79 & nonanal & 16.12 & 142 & 0.04 & 0.03 & 0.02 & 0.12 & 0.07 & 0.05 & 0.03 & 0.03 & & & 0.07 & 0.04 & 0.06 \\
\hline 80 & C4-benzene & 16.24 & 134 & & & & 0.03 & & & & & & & & & \\
\hline 81 & phosphoric acid, triethyl ester & 16.41 & 182 & & & & & & & & & & & & & \\
\hline 82 & C4-benzene & 16.43 & 134 & & & & & & & & & & & & 0.05 & 0.04 \\
\hline 83 & trans methyl decalin & 16.50 & 152 & & & & & & & & & & & & & \\
\hline 84 & C4-benzene & 16.55 & 134 & & & & & & & & & & & 0.02 & 0.05 & 0.04 \\
\hline 85 & cis methyl decalin & 16.74 & 152 & & & & & & & & & & & & & \\
\hline 86 & C5-benzene & 16.79 & 148 & & & & & & & & & & & & & \\
\hline 87 & methyl indan & 17.14 & 132 & & & & & & & & & & & & & \\
\hline 88 & 0,0-diethyl-S-ethyl phosphorothioate & 17.16 & 198 & & & & & & & & & & & & & \\
\hline 89. & methyl indan & 17.29 & 132 & & & & 0.11 & & & & & & & & & \\
\hline 90 & trichlorobenzene & 17.64 & 180 & & & & & & & & & & & & & \\
\hline 91 & 1-dodecene & 17.66 & 168 & & & & & & & & & & & & & \\
\hline 92 & 1-(4-methylphenyl) ethanone & 17.67 & 134 & & & & & & & & & & & & & \\
\hline 93 & $\mathrm{~N}, \mathrm{~N}-\mathrm{d}$ b butyl-1-butanamine & \begin{tabular}{|l|}
17.72 \\
\end{tabular} & 142 & & & & & & & & & & & & & \\
\hline 94 & n-dodecane & 17.80 & 170 & & & & 0.03 & & & & & & & & & \\
\hline 95 & naphthalene & \begin{tabular}{|l|l|}
17.79 \\
\end{tabular} & 128 & 0.02 & 0.01 & 0.02 & 0.15 & 0.03 & 0.04 & 0.20 & 0.15 & & & 0.05 & 0.18 & 0.16 \\
\hline 96 & C5-benzene & \begin{tabular}{|l|l|}
17.87 \\
\end{tabular} & 148 & & & & & & & & & & & & & \\
\hline 97 & decanal & 17.90 & 156 & 0.03 & 0.02 & 0.01 & 0.13 & 0.07 & 0.05 & & & & & 0.03 & & 0.04 \\
\hline 98 & benzothiophene & 17.90 & 134 & & & & & & & & & & & & & \\
\hline 99 & 1,4-oxathiane, 4,4-dioxide & 18.35 & 136 & & & & & & & & & & & & & \\
\hline 100 & benzothiazole & 18.40 & 135 & & & & & & & & & & & & & \\
\hline 101 & benzene propanenifrile & 18.53 & 131 & & & & & & & & & & & & & \\
\hline 102 & dimethyl indan & 18.80 & 146 & & & & & & & & & & & & & \\
\hline 103 & 3-tetradecene & 18.85 & 196 & & & & & & & & & & & & & \\
\hline 104 & $\mathrm{~N}$-butyl-N-nitroso $\mathrm{N}$-butanamine & 18.91 & 158 & & & & & & & & & & & & & \\
\hline 105 & 1-tridecene & $\mid 19.26$ & 182 & & & & & & & & & & & & & \\
\hline 106 & n-tridecane & 19.38 & 184 & & & & & & & & & & & & & \\
\hline 107 & N.N-dibutyl formamide & 19.51 & 157 & & & & & & & & & & & & & \\
\hline 108 & 2-methyl naphthalene & 19.61 & 142 & & & & 0.05 & & & 0.05 & 0.04 & & & 0.01 & 0.06 & 0.03 \\
\hline 109 & phthalate & 19.80 & 390 & & & & & & & & & & & & & \\
\hline 110 & 1,3-isobenzofurandione & 19.81 & 148 & & & & 0.05 & & & & & & & & & \\
\hline 111 & 1-methyl naphthalene & 19.91 & 142 & & & & 0.04 & & & & & & & & & \\
\hline 112 & 1-methyl-4-(propylthio) benzene & 20.37 & 166 & & & & & & & & & & & & & \\
\hline 113 & 1-tetradecene & 20.76 & 196 & & & & & & & & & & & & & \\
\hline 114 & n-tetradecane & 20.86 & 198 & & & & & & & & & & & & & \\
\hline 115 & bipheny & 20.87 & 154 & 0.01 & 0.00 & 0.01 & & 0.02 & 0.01 & 0.07 & 0.04 & & & 0.01 & 0.04 & 0.07 \\
\hline 116 & $1,1^{\prime}$-oxy bis(benzene) & 21.17 & 186 & & & & 0.04 & & & 0.06 & 0.04 & & & 0.01 & 0.03 & 0.09 \\
\hline 117. & 2,4,6-trichloroaniline & 21.20 & 195 & & & 0.01 & 0.03 & & 0.01 & 0.01 & 0.02 & & & & 0.02 & 0.02 \\
\hline 118 & C2-naphthalene & 21.28 & 156 & & & & & & & & & & & & & \\
\hline 119 & C2-naphthalene & 21.51 & 156 & & & & & & & 0.02 & 0.02 & & & & 0.02 & \\
\hline 120 & C2-naphthalene & 21.56 & 156 & & & & & & & & & & & & & \\
\hline 121 & tribromobenzene & 21.62 & 312 & & & & & & & & & & & & & \\
\hline 122 & 6.10-dimethyl-5,9-undecadien-2-one & 21.65 & 194 & & & & & & & & & & & & & \\
\hline 123 & 2,6-di-t-butyl-2,5-cyclohexadiene-1,4-dione & 22.06 & 220 & & & & & & & & & & & & & \\
\hline 124 & 1-pentadecene & 22.16 & 210 & & & & & & & & & & & & & \\
\hline 125 & n-pentadecane & 22.26 & 212 & & & & & & & & & & & & & \\
\hline 126 & methyl biphenyl & 22.30 & 168 & & & & & & & & & & & & & \\
\hline 127| & 1,2-dihydroacenaphthylene & 22.50 & 168 & & & & & & & & & & & & & \\
\hline
\end{tabular}


Air Monitoring Results

Sample number and room number at the top of each data column

\begin{tabular}{|c|c|c|c|c|c|c|c|c|c|c|c|c|c|c|c|c|}
\hline & Compound & & & $\begin{array}{c}56 \\
E 303\end{array}$ & \begin{tabular}{|l|}
57 \\
5303
\end{tabular} & \begin{tabular}{|l|}
54 \\
\end{tabular} & \begin{tabular}{|l|}
164 \\
$E 305$
\end{tabular} & \begin{tabular}{|r|}
55 \\
53
\end{tabular} & $\begin{array}{c}53 \\
E 306\end{array}$ & $\begin{array}{c}60 \\
E 308\end{array}$ & \begin{tabular}{|c|}
6 \\
\end{tabular} & \begin{tabular}{|c|}
134 \\
$E 308$ \\
\end{tabular} & \begin{tabular}{|c|}
58 \\
$E 309$ \\
\end{tabular} & \begin{tabular}{|c|}
59 \\
$E 309$ \\
\end{tabular} & \begin{tabular}{|c|}
63 \\
$E 310$ \\
\end{tabular} & $\begin{array}{c}64 \\
E 311\end{array}$ \\
\hline & Tentative Identification & RT & MW & $n$ & $\mathrm{~s}$ & s & $\frac{\mathrm{n}}{\mathrm{ne}}$ & $n$ & $\frac{\mathrm{EN} 00}{\mathrm{~m}}$ & \begin{tabular}{|l} 
Lovo \\
$\mathrm{s}$
\end{tabular} & $n$ & & $\mathbf{n}$ & 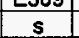 & $\mathrm{m}$ & $\mathrm{m}$ \\
\hline 128 & C3-naphthalene & 22.65 & 170 & & & & & & & & & & & & & \\
\hline 129 & phenyl maleic anhydride & 22.90 & 214 & & & & 0.03 & & & & & & & & & \\
\hline 130 & dibenzofuran & 22.93 & 168 & & & & & & & & & & & & & \\
\hline 131 & 1 -hexadecene & 22.47 & 224 & & & & & & & & & & & & & \\
\hline 932 & In-hexadecane & 23.57 & 226 & & & & & & & & & & & & & \\
\hline 133 & benzenedicarboxylic acid, diethyl ester & 23.64 & 222 & & & & & & & & & & & & & \\
\hline 134 & 1,3-dibromo-2,2-bis(bromomethyl) propane & 24.17 & 384 & & & & & & & & & & & & & \\
\hline 135 & diphenyl diazene & 24.23 & 182 & & & & & & & & & & & & & \\
\hline 136 & benzophenone & 24.30 & 182 & & & & & & & & & & & & & \\
\hline 437 & 1-heptadecene & 24.71 & 238 & & & & & & & & & & & & & \\
\hline 138 & n-heptadecane & 24.81 & 240 & & & & & & & & & & & & & \\
\hline 139 & $\mathrm{~N}$-(phenyimethylene) benzamine & 24.83 & 181 & & & & & & & & & & & & & \\
\hline 140 & 9H-fluoren-9-one & 25.28 & 180 & & & & & & & & & & & & & \\
\hline 141 & tribromobenzamine & 25.39 & 327 & & & & & & & & & & & & & \\
\hline 142 & trimethyl indan & 25.45 & 160 & & & & 0.11 & & & & 0.06 & & & & & \\
\hline 143 & dibenzothiophene & 25.58 & 184 & & & & & & & & & & & & & \\
\hline 144 & tetrabromobenzene & \begin{tabular}{|l|l|}
25.86 \\
\end{tabular} & 390 & & & & & & & & & & & & & \\
\hline 145 & n-octadecane & 25.88 & 254 & & & & & & & & & & & & & \\
\hline 146 & phenanthrene/anthracene & 26.37 & 178 & & & & 0.04 & 0.05 & 0.00 & & & & & & 0.01 & 0.01 \\
\hline 147 & methyl dibenzothiophene & 26.98 & 198 & & & & & & & & & & & & & \\
\hline 148 & n-nonadecane & 27.04 & 268 & & & & & & & & & & & & & \\
\hline 149 & 2-methyl anthracene/phenanthrene & 27.48 & 192 & & & & & & & & & & & & & \\
\hline 150 & 1-methyl anthracene/phenanthrene & 27.57 & 192. & & & & & & & & & & & & & \\
\hline 151 & 2,6-dibutyl-2,5-cyclohexadien-1,4-dione & 31.30 & 220 & & & & & & & & & & & & & \\
\hline 152 & Total PCB's & & & & & $t$ & & 4.90 & 0.05 & & & & & & & \\
\hline & & & & & & & & & & & & & & & & \\
\hline & & & & & & & & & & & & & & & & \\
\hline & & & & & & & & & & & & & & & & \\
\hline & & & & & & & & & & & & & & & & \\
\hline & & & & & & & & & & & & & & & & \\
\hline & & & & & & & & & & & & & & & & \\
\hline & & & & & & & & & & & & & & & & \\
\hline & & & & & & & & & & & & & & & & \\
\hline & & & & & & & & & & & & & & & & \\
\hline & & & & & & & & & & & & & & & & \\
\hline & & & & & & & & & & & & & & & & \\
\hline & & & & & & & & & & & & & & & & \\
\hline & & & & & & & & & & & & & & & & \\
\hline & & & & & & & & & & & & & & & & \\
\hline & & & & & & & & & & & & & & & & \\
\hline & & & & & & & & & & & & & & & & \\
\hline & & & & & & & & & & & & & & & & \\
\hline & & & & & & & & & & & & & & & & \\
\hline & & & & & & & & & & & & & & & & \\
\hline & t & & & & & & & & & & & & & & & \\
\hline & & & & & & & & & & & & & & & & \\
\hline & & & & & & & & & & & & & & & & \\
\hline & 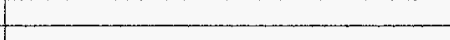 & & & & & $\cdot$ & & & & & & & & & & \\
\hline & & & & & & & & & & & & & & & & \\
\hline & & & & & & & & & & & & & & & & \\
\hline & & & & & & & & & & & & & & & & \\
\hline & & & 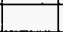 & & & & & & & & & & & & & \\
\hline & & & & & & & & & & & & & & & & \\
\hline & & & & & & & & & & & & & & & & \\
\hline & & & & & & & & & & & & & & & & \\
\hline & & & $\ldots$ & & & & & & & & & & & & & - \\
\hline & & & & & & & & & & & & & & & & 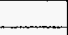 \\
\hline & & & & & & & & & & & & & & & & $\ldots$ \\
\hline & & & & & & & & & & & & & & & & \\
\hline & & & & & & & & & & & & & & & & \\
\hline & & & & & & & & & & & & & & & & \\
\hline & & & $b=$ & & & & & 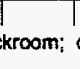 & vay & onwa & & & & & & \\
\hline
\end{tabular}




\begin{tabular}{|c|c|c|c|c|c|c|c|c|c|c|c|c|c|c|c|c|}
\hline & & & & 62 & 65 & 135 & 136 & 66 & 165 & 67 & 73 & 71 & 72 & 70 & \begin{tabular}{|c|}
69 \\
\end{tabular} & \begin{tabular}{|c|}
68 \\
$E 321$
\end{tabular} \\
\hline & Compound & & & E311 & E313 & $E 313$ & & E314 & E314 & E314 & E317 & E318 & & E319 & E320 & $E 321$ \\
\hline & Tentative Identification & RT & MW & s & $\mathrm{m}$ & & 0 & $n$ & $n$ & s & $\mathrm{m}$ & $\mathrm{m}$ & 0 & $\mathrm{~m}$ & $\mathrm{~m}$ & $n$ \\
\hline 1 & sulfur dioxide & 1.55 & 64 & & & & & & & & & & & & & \\
\hline 2 & 2-propanone & 1.89 & 58 & & & & & & & & & & & & & \\
\hline 3 & 1-fluoro-1,1-dichloroethane & 1.95 & 116 & & & & & & & & & & & & & \\
\hline 4 & N-ethyl ethanamine & 2.36 & 73 & & & & & & & & & & & & & \\
\hline 5 & 2-butanone & 2.56 & 72 & & & & & & & & & & & & & \\
\hline 6 & $\mathrm{~N}$-ethyl-N-methyi ethanamine & 2.94 & 87 & & & & & & & & & & & & & \\
\hline 7 & dichloromethane & 3.15 & 84 & & & & & 0.04 & & & & & & & & \\
\hline 8 & N-(1-methylethyl)-2-propanamine & 3.59 & 101 & & & & & & & & & & & & & \\
\hline 9 & benzene & 3.64 & 78 & 0.01 & & & & & 0.36 & 0.00 & & & & & 0.04 & \\
\hline 10 & 2-pentanone & 4.08 & 86 & & & & & & & & & & & & & \\
\hline 11 & trichloroethene & 4.47 & 130 & & & & & & 0.05 & & & & & & & \\
\hline 12 & N-(1-methylethylidene)-2-propanamine & 4.62 & 99 & & & & & & & & & & & & & \\
\hline 13 & toluene & 6.97 & 92 & 0.16 & 0.00 & & & 0.00 & 0.51 & 0.05 & & & & & 0.11 & \\
\hline 14 & N-methyl-N-(1-methylethyl)-2-propanamine & 7.27 & 115 & & & & & & & & & & & & & \\
\hline 15 & 3-hexanone & 7.75 & 100 & & & & & & & & & & & & & \\
\hline 16 & 2-hexanone & 7.92 & 100 & & & & & & & & & & & & & \\
\hline 17 & n-octane & 8.29 & 114 & & & & & & & & & & & & & \\
\hline 18 & hexanal & 8.33 & 100 & & & & & & & & & & 0.03 & & & \\
\hline 19 & tetrachloroethene & 8.79 & 164 & 0.01 & & & & & & 0.00 & & & & & 0.01 & \\
\hline 20 & melhyl ethyl disulfide & 9.68 & 108 & & & & & & & & & & & & & \\
\hline 21 & chlorobenzene & 10.05 & 112 & & & & & & & & & & & & & \\
\hline 22 & ethyl benzene & 10.57 & 106 & 0.05 & 0.00 & & & 0.00 & 0.07 & 0.01 & & & & & 0.03 & \\
\hline 23 & $p, m-x y$ lene & 10.81 & 106 & 0.17 & 0.01 & & & 0.01 & 0.21 & 0.03 & & & & & 0.08 & \\
\hline 24 & 1,4-oxathiane & 11.00 & 104 & & & & & & & & & & & & & \\
\hline 25 & styrene & 11.30 & 118 & & & & & & & & & & & & & \\
\hline 26 & 3-heptanone & 11.37 & 114 & & & & & & & & & & & & & \\
\hline 27 & $0-x y l e n e$ & 11.49 & 106 & 0.06 & 0.00 & & & 0.00 & 0.24 & 0.01 & & & & & 0.04 & \\
\hline 28 & heptanal & 11.60 & 114 & & & & & & & & & & & & & \\
\hline 29 & n-nonane & 11.67 & 128 & 0.01 & 0.01 & & & & & 0.00 & & & & & & \\
\hline 30 & C3-benzene & 12.20 & 120 & & & & & & & & & & & & & \\
\hline 31 & N-butylidene-1-butanamine & 12.28 & 127 & & & & & & & & & & & & & \\
\hline 32 & C3-benzene & 12.33 & 120 & & & & & & & & & & & & 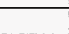 & \\
\hline 33 & 3-methyl nonane & 12.51 & 142 & & & & & & & & & & & & & \\
\hline 34 & pinene & 12.61 & 136 & & & & & & & & & & & & & \\
\hline 35 & 2-methyl cyclohexanol & 12.62 & 114 & & & & & & & & & & & & & \\
\hline 36 & 3-octanone & 12.71 & 128 & & & & & & & & & & & & & \\
\hline 37 & 2-ethyl hexanal & 13.02 & 128 & & & & & & & & & & 0.01 & & & \\
\hline 38 & 1-chloro-2-methyl benzene & 13.03 & 126 & & & & & & & & & & & & & \\
\hline 39 & propyl benzene & 13.05 & 120 & 0.02 & & & & & & & & & & & & \\
\hline 40 & $\mathrm{~N}$-butyl-1-butanamine & 13.17 & 129 & & & & & & & & & & & & & \\
\hline 41 & benzaldehyde & 13.18 & 106 & 0.03 & 0.01 & & 0.16 & & 0.48 & 0.01 & & & 0.07 & 0.01 & & \\
\hline 42 & C3-benzene & 13.25 & 120 & 0.08 & 0.05 & & & & & & & & & & 0.05 & \\
\hline 43 & C3-benzene & 13.41 & $\mid 120$ & 0.02 & 0.01 & & & & & & & & & & 0.01 & \\
\hline 44 & phenol & 13.57 & 94 & & & & 0.05 & & 0.12 & & & & 0.02 & & & \\
\hline 45 & aniline & 13.61 & 93 & & & & & & & & & & & & & \\
\hline 46 & C3-benzene & 13.68 & 120 & 0.03 & & & & & & & & & & & & \\
\hline 47 & 6-methyl-5-hepten-2-one & 13.69 & 126 & & & & & & & & & & 0.02 & & & \\
\hline 48 & benzonitrile & 13.72 & 103 & & & & & & & & & & 0.02 & & 0.03 & \\
\hline 49 & 1-decene & 13.83 & 140 & & & & & & & & & & & & & \\
\hline 50 & C3-benzene & 13.99 & 120 & 0.12 & 0.06 & & & & & & & & & & 0.07 & \\
\hline 51 & n-decane & 14.07 & 142 & 0.03 & 0.02 & & & & & & & & & & & \\
\hline 52 & octanal & 14.09 & 128 & & & & & & 0.09 & & & & 0.03 & & & \\
\hline 53 & dichlorobenzene & 14.40 & 146 & & & & & & & & & & & & & \\
\hline 54 & 4-methyl decane & 14.56 & 156 & & & & & & & & & & & & & \\
\hline 55 & C3-benzene & 14.57 & 120 & & & & & & & & & & & & & \\
\hline 56 & C4-benzene & 14.60 & 134 & 0.04 & & 0.03 & & & & & & & & & 0.03 & \\
\hline 57 & 2-ethyl hexanol & 14.64 & 130 & & 0.04 & & & & 0.05 & 0.03 & 0.04 & & 0.08 & & & \\
\hline 58 & limonene & 14.75 & 136 & 0.01 & 0.04 & 0.03 & & & & & & & & & & \\
\hline 59 & benzene methanol & 14.77 & 108 & & & & & & & & & & & & & \\
\hline $60 \mid$ & indan & 14.87 & 118 & & & & & & & & & & & & & \\
\hline 61 & dichlorobenzene & 14.89 & 146 & & & & & & & & & & & & & \\
\hline 62 & $\mathrm{~N}$-ethyl-N-(1-methylethyl)-2-propanamine & 15.07 & 129 & & & & & & & & & & & & & \\
\hline $63 \mid$ & C4-benzene & 15.10 & 134 & & & & & & & & & & & & 0.02 & \\
\hline
\end{tabular}




\begin{tabular}{|c|c|c|c|c|c|c|c|c|c|c|c|c|c|c|c|c|}
\hline & & & & 62 & 65 & 135 & 136 & 66 & 165 & 67 & 73 & 71 & 72 & 70 & 69 & 68 \\
\hline & Compound & & & E311 & E313 & E313 & & E394 & E314 & E314 & E317 & E348 & & $E 319$ & $E 320$ & $E 321$ \\
\hline & Tentative identification & RT & MW & $\mathbf{s}$ & $m$ & & 0 & $n$ & $n$ & s & $m$ & $m$ & 0 & $m$ & $m$ & $n$ \\
\hline 64 & 5-methyl decane & 15.21 & 156 & & & & & & & & & & & & & \\
\hline 65 & 4-methyl decane & 15.29 & 156 & & & & & & & & & & & & & \\
\hline 66 & C4benzene & 15.30 & 134 & & & & & & & & & & & & & \\
\hline 67 & 2-methyl decane & 15.34 & 156 & & & & & & & & & & & & & \\
\hline 68 & 1,4-dithiane & 15.39 & 120 & & & & & & & & & & & & & \\
\hline 69 & acetophenone & 15.47 & 120 & 0.01 & & 0.01 & 0.17 & & 0.53 & 0.01 & & & 0.06 & 0.01 & & \\
\hline 70 & C4-benzene & 15.47 & 134 & & & & & & & & & & & & & \\
\hline 71 & 3-methyl decane & 15.48 & 156 & & & & & & & & & & & & & \\
\hline 72 & methyl benzaldehyde & 15.51 & 120 & & & & & & & & & & & & & \\
\hline 73 & C4-benzene & 15.64 & 134 & & & & & & & & & & & & & \\
\hline 74 & CA-benzene & 15.72 & 134 & & & & & & & & & & & & & \\
\hline 75 & A,A-dimethyl benzene methanol & 15.80 & 136 & & & & & & & & & & & & & \\
\hline 76 & C4-benzene & 15.86 & 134 & & & & & & & & & & & & 0.02 & \\
\hline 77 & 1-undecene & 15.95 & 154 & & & & & & & & & & & & & \\
\hline 78 & n-undecane & 16.05 & 156 & 0.03 & 0.01 & & & & & 0.01 & & & & & 0.01 & \\
\hline 79 & nonanal & 16.12 & 142 & 0.01 & & & & & 0.23 & 0.02 & & & 0.05 & & & \\
\hline 80 & C4-benzene & 16.24 & 134 & & & & & & & & & & & & & \\
\hline 81 & phosphoric acid, triethyl ester & 16.41 & 182 & & & & & & & & & & & & & \\
\hline 82 & C4-benzene & 16.43 & 134 & & & & & & & & & & & & & \\
\hline 83 & trans methyl decalin & 16.50 & 152 & & & & & & & & & & & & & \\
\hline 84 & C4-benzene & 16.55 & 134 & & & & & & & & & & & & & \\
\hline 85 & cis methyl decalin & 16.74 & 152 & & & & & & & & & & & & & \\
\hline 86 & C5-benzene & 16.79 & 148 & & & & & & & & & & & & & \\
\hline 87 & methyl indan & 17.14 & 132 & & & & & & & & & & & & & \\
\hline 88 & O,O-diethyl-S-ethyl phosphorathioate & 17.16 & 198 & & & & & & & & & & & & & \\
\hline 89 & methyl indan & 17.29 & 132 & & & & & & & & & & & & & \\
\hline 90| & trichlorobenzene & 17.64 & 180 & & & & & & & & & & & & & \\
\hline 91 & 1-dodecene & 17.66 & 168 & & & & & & & & & & & & & \\
\hline 92 & 1-(4-methylphenyl) ethanone & 17.67 & 134 & & & & & & & & & & & & & \\
\hline 93 & N.N-dibutyl-1-butanamine & 17.72 & 142 & & & & & & & & & & & & & \\
\hline 94 & n-dodecane & 17.80 & 170 & & & & & & & & & & & & & \\
\hline 95 & naphthalene & 17.79 & 128 & 0.05 & 0.04 & & & & & 0.01 & & & & & 0.02 & \\
\hline 96 & C5-benzene & 17.87 & 148 & & & & & & & & & & & & & \\
\hline 97 & decanal & 17.90 & 156 & 0.01 & & & & & 0.20 & 0.02 & & & 0.05 & & & \\
\hline 98 & benzothiophene & 17.90 & 134 & & & & & & & & & & & & & \\
\hline 99 & 1,4-oxathiane, 4,4-dioxide & 18.35 & 136 & & & & & & & & & & & & & \\
\hline 100 & benzothiazole & 18.40 & 135 & & & & & & & & & & & & & $=$ \\
\hline 101 & benzene propanenitrile & 18.53 & 131 & & & & & & & & & & & & & \\
\hline 102 & dimethyl indan & 18.80 & 146 & & & & & & & & & & & & & \\
\hline 103 & 3-tetradecene & 18.85 & 196 & & & & & & & & & & & & & \\
\hline 104 & $\mathrm{~N}$-butyl- $\mathrm{N}$-nitroso $\mathrm{N}$-butanamine & 18.91 & 158 & & & & & & & & & & & & & \\
\hline 105 & 1-tridecene & 19.26 & 182 & & & & & & & & & & & & & \\
\hline 106 & n-tridecane & 19.38 & 184 & & & & & & & & & & & & & \\
\hline 107 & N,N-dibutyl formamide & 19.51 & 157 & & & & & & & & & & & & & \\
\hline 108. & 2-methyl naphthalene & 19.61 & 142 & 0.01 & 0.01 & & & & & & & & & & 0.01 & \\
\hline 109 & phthalate & 19.80 & 390 & & & & & & & & & & & & & \\
\hline 110 & 1,3-isobenzofurandione & 19.81 & 148 & & & & & & 0.03 & & & & & & & \\
\hline 111 & 1-methyt naphthalene & 19.91 & 142 & & & & & & & & & & & & & \\
\hline 112 & 1-methy-4-(propylthio) benzene & 20.37 & 166 & & & & & & & & & & & & & \\
\hline 113 & 1-tetradecene & 20.76 & 196 & & & & & & & & & & & & & \\
\hline 114 & n-tetradecane & 20.86 & 198 & & & & & & & & & & & & & \\
\hline 115 & biphenyl & 20.87 & 154 & 0.01 & 0.02 & & & & & 0.00 & & & & & 0.01 & \\
\hline 116 & $1,1^{\prime}$-oxy bis(benzene) & 21.17 & 186 & 0.02 & 0.03 & & & & & & & & & & 0.01 & \\
\hline 117 & 2,4,6-trichloroaniline & 21.20 & 195 & & & & & & & & & & & & & \\
\hline 118 & C2-naphthalene & 21.28 & 156 & & & & & & & & & & & & & \\
\hline 119 & C2-naphthalene & 21.51 & 156 & & & & & & & & & & & & & \\
\hline 120 & C2-naphthalene & 21.56 & 156 & & & & & & & & & & & & & \\
\hline 121 & tribromobenzene & 21.62 & 312 & & & & & & & & & & & & & \\
\hline 122 & 6,10-dimethyl-5,9-undecadien-2-one & 21.65 & 194 & & & & & & & & & & & & & \\
\hline 123 & 2,6-di-t-butyl-2,5-cyclohexadiene-1,4-dione & 22.06 & 220 & & & & & & & & & & & & & \\
\hline 124 & 1-pentadecene & 22.16 & 210 & & & & & & & & & & & & & \\
\hline 125 & n-pentadecane & 22.26 & 212 & & & & & & & & & & & & & \\
\hline 126 & methyl biphenyl & 22.30 & 168 & & & & & & & & & & & & & \\
\hline 127 & 1,2-dihydroacenaphthylene & 22.50 & 168 & & & & & & & & & & & & & \\
\hline
\end{tabular}


Air Monitoring Results

Sample number and room number at the top of each data column

\begin{tabular}{|c|c|c|c|c|c|c|c|c|c|c|c|c|c|c|c|c|}
\hline & & & & 62 & 65 & 135 & 136 & 66 & 165 & 67 & 73 & 71 & 72 & 70 & 69 & 68 \\
\hline & Compound & & & E311 & $E 313$ & E313 & & E314 & $E 314$ & $E 314$ & $E 317$ & E318 & & E319 & $E 320$ & E321 \\
\hline & Tentative Identification & RT & MW & $\mathrm{s}$ & $\mathrm{m}$ & & 0 & $\mathrm{n}$ & $n$ & $\mathbf{s}$ & $\mathrm{m}$ & $\mathrm{m}$ & $\circ$ & $\mathrm{m}$ & $\mathrm{m}$ & $n$ \\
\hline 128 & C3-naphthalene & 22.65 & 170 & & & & & & & & & & & & & \\
\hline 129 & phenyl maleic anhydride & 22.90 & 214 & & & & 0.02 & & 0.07 & & & & & & & \\
\hline 130 & dibenzofuran & 22.93 & 168 & & & & & & & & & & & & & \\
\hline 131 & 1-hexadecene & 22.47 & 224 & & & & & & & & & & & & & \\
\hline 132 & n-hexadecane & 23.57 & 226 & & & & & & & & & & & & & \\
\hline 133 & benzenedicarboxylic acid, diethyl ester & 23.64 & 222 & & & & & & & & & & & & & \\
\hline 134 & 1,3-dibromo-2,2-bis(bromomethyl) propane & 24.17 & 384 & & & & & & & & & & & & & \\
\hline 135 & diphenyl diazene & 24.23 & 182 & & & & & & & & & & & & & \\
\hline 136 & benzophenone & \begin{tabular}{|l|}
24.30 \\
\end{tabular} & 182 & & & & & & & & & & & & & \\
\hline 137 & 1-heptadecene & 24.71 & 238 & & & & & & & & & & & & & \\
\hline 138 & In-hepladecane & 24.81 & 240 & & & & & & & & & & & & & \\
\hline 139 & $\mathrm{~N}$-(phenylmethylene) benzamine & 24.83 & 181 & & & & & & & & & & & & & \\
\hline 140 & 9H-fluoren-9-one & 25.28 & 180 & & & & & & & & & & & & & \\
\hline 141 & tribromobenzamine & 25.39 & 327 & & & & & & & & & & & & & \\
\hline 142 & trimethyl indan & 25.45 & 160 & 0.04 & & & & 0.01 & & & & & & & & \\
\hline 143 & dibenzothiophene & 25.58 & 184 & & & & & & & & & & & & & \\
\hline 144 & tetrabromobenzene & 25.86 & 390 & & & & & & & & & & & & & \\
\hline 145 & n-octadecane & 25.88 & 254 & & & & & & & & & & & & & \\
\hline 146 & phenanthrene/anthracene & 26.37 & 178 & & & & & 0.04 & & & & & & & 0.02 & \\
\hline 147 & methyl dibenzothiophene & 26.98 & 198 & & & & & & & & & & & & & \\
\hline 148 & n-nonadecane & 27.04 & 268 & & & & & 0.02 & & & & & & & & \\
\hline 149 & 2-methyl anthracene/phenanthrene & 27.48 & 192 & & & & & & & & & & & & & \\
\hline 150 & 1 -methyl anthracene/phenanthrene & 27.57 & 492 & & & & & & & & & & & & & \\
\hline 151 & 2,6-dibutyl-2,5-cyclohexadien-1,4-dione & 31.30 & 220 & & & & & & 0.65 & & & & & & & \\
\hline 152 & Total PCB's & & & & & & & 3.00 & & & & & & & & \\
\hline & & & & & & & & & & & & & & & & \\
\hline & & & & & & & & & & & & & & & & \\
\hline & & & & & & & & & & & & & & & & \\
\hline & & & & & & & & & & & & & & & & \\
\hline & & & & & & & & & & & & & & & & \\
\hline & & & & & & & & & & & & & & & & \\
\hline & & & & & & & & & & & & & & & & \\
\hline & & & & & & & & & & & & & & & & \\
\hline & & & & & & & & & & & & & & & & \\
\hline & & & & & & & & & & & & & & & & \\
\hline & & & & & & & & & & & & & & & & \\
\hline & & & & & & & & & & & & & & & & \\
\hline & & & & & & & & & & & & & & & & \\
\hline & & & & & & & & & & & & & & & & \\
\hline & & & & & & & & & & & & & & & & \\
\hline & & & & & & & & & & & & & & & & \\
\hline & & & & & & & & & & & & & & & & \\
\hline & & & & & & & & & & & & & & & & \\
\hline & & & & & & & & & & & & & & & & \\
\hline & & & & & & & & & & & & & & & & \\
\hline & - & & & & & & & & & & & & & & & \\
\hline & & & & & & & & & & & & & & & & \\
\hline & & & & & & & & & & & & & & & & \\
\hline & & & & & & & & & & & & & & & & \\
\hline & & & & & & & & & & & & & & & & \\
\hline & & & & & & & & & & & & & & & & \\
\hline & & & & & & & & & & & & & & & & \\
\hline & & & & & & & & & & & & & & & & \\
\hline & & & & & & & & & & & & & & & & \\
\hline & & & & & & & & & & & & & & & & \\
\hline & & & & & & & & & & & & & & & . & 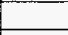 \\
\hline & & & & & & & & & & & & & & & & \\
\hline & & & & & & & & & & & & & & & & \\
\hline & & & & & & & & & & & & & & & & 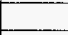 \\
\hline & & & & & & & & & & & & & & & & $\ldots$ \\
\hline & & & & & & & & & & & & & & & & + \\
\hline 1 & & & & & & & & . & & & & & & & & \\
\hline
\end{tabular}


Air Monitoring Results

Sample number and room number at the top of each data column

\begin{tabular}{|c|c|c|c|c|c|c|c|c|c|c|c|c|c|c|c|c|}
\hline & & & & 78 & 79 & 74 & 75 & 76 & 77 & 132 & 85 & 86 & 84 & 130 & 81 & 82 \\
\hline & Compound & & & $E 403$ & $E 403$ & E405 & E405 & $E 408$ & $E 408$ & E408 & $E 409$ & E409: & $E 411$ & E411 & $E 412$ & \\
\hline & Tentative Identification & RT & MW & s & $n$ & ne & sw & ne & sw & & e & $w$ & drway & & $\mathbf{s}$ & 0 \\
\hline 1 & sulfur dioxide & 1.55 & 64 & & & & & & & & & & & & & \\
\hline 2 & 2-propanone & 1.89 & 58 & & & & & & & & & & & & & \\
\hline 3 & 1-fluoro-1, 1-dichloroethane & 1.95 & 116 & & & & & & & & & & & & & \\
\hline 4 & Nethyl ethanamine & 2.36 & 73 & & & & & & & & & & & & & \\
\hline 5 & 2-butanone & 2.56 & 72 & & & & & & & & & & & & & \\
\hline 6 & $\mathrm{~N}$-ethyl-N-methyl ethanamine & 2.94 & 87 & & & & & & & & & & & & & \\
\hline 7 & dichloromethane & 3.15 & 84 & & & & & & & 0.22 & & & & & & \\
\hline 8 & $\mathrm{~N}$-(1-methylethyl)-2-propanamine & 3.59 & 101 & & & & & & & & & & & & & \\
\hline 9 & benzene & 3.64 & 78 & & & & & & & & & & & & & \\
\hline 10 & 2-pentanone & 4.08 & 86 & & & & & & & 0.08 & & & & & & \\
\hline 11 & trichlorcethene & 4.47 & 130 & & & & & & & & & & & & & \\
\hline 12 & $\mathrm{~N}$-(1-methylethylidene)-2-propanamine & 4.62 & 99 & & & & & & & & & & & & & \\
\hline 13 & toluene & 6.97 & 92 & & 0.06 & 0.07 & 0.07 & 0.15 & 0.21 & 0.15 & 0.08 & 0.17 & 0.29 & 0.02 & & \\
\hline 14 & $\mathrm{~N}$-methyl-N-(1-methylethyl)-2-propanamine & 7.27 & 115 & & & & & & & & & & & & & \\
\hline 15 & 3-hexanone & 7.75 & 100 & & & & & & & & & & & & & \\
\hline 16 & 2-hexanone & 7.92 & 100 & & & & & & & & & & & & & \\
\hline 17 & n-octane & 8.29 & 114 & & & & & & & & & & & & & \\
\hline 18 & hexanal & 8.33 & 100 & & & & & & & & & & & & & \\
\hline 19 & tetrachloroethene & 8.79 & 164 & & & & & & & & & & & & & \\
\hline 20 & methyl ethyl disulfide & 9.68 & 108 & & & & & & & & & & & & & \\
\hline 21 & chlorobenzene & 10.05 & 112 & & & & & & & & & & & & & \\
\hline 22 & ethyl benzene & 10.57 & 106 & & & 0.01 & & & & & 0.01 & 0.03 & 0.06 & & & \\
\hline 23 & $p, m$-xylene & 10.81 & 106 & & 0.02 & 0.03 & 0.03 & 0.07 & & 0.06 & 0.06 & 0.11 & 0.23 & & & \\
\hline 24 & 1,4-oxathiane & 11.00 & 104 & & & & & & & & & & & & & \\
\hline 25 & styrene & 11.30 & 118 & & & & & & & & & & & & & \\
\hline 26 & 3-heptanone & 11.37 & 114 & & & & & & & & & & & & & \\
\hline 27 & o-xylene & 11.49 & 106 & & & 0.01 & & & & 0.02 & 0.03 & 0.04 & 0.09 & & & \\
\hline 28 & hepianal & 11.60 & 114 & & & & & & & & & & & & & \\
\hline 29 & n-nonane & 11.67 & 128 & & & & & & & & & & & & & \\
\hline 30 & C3-benzene & 12.20 & 120 & & & & & & & & & & & & & \\
\hline 31 & $N$-butylidene-1-butanamine & 12.28 & 127 & & & & & & & & & & & & & \\
\hline 32 & |C3-benzene & 12.33 & 120 & & & & & & & & & & & & & \\
\hline 33 & 3-methyl nonane & 12.51 & 142 & & & & & & & & & & & & & \\
\hline 34 & pinene & 12.61 & 136 & & & & & & & & & 0.02 & & & & \\
\hline 35 & 2-methyl cyclohexanol & 12.62 & 114 & & & & & & & & & & & & & \\
\hline 36 & 3-octanone & 12.71 & 128 & & & & & & & & & & & & & \\
\hline 37 & 2-ethyl hexanal & 13.02 & 128 & & & & & & & & & & & & & \\
\hline 38 & 1-chloro-2-methyl benzene & 13.03 & 126 & & & & & & & & & & & & & \\
\hline 39 & propyl benzene & 13.05 & 120 & & & & & & & & & & & & & \\
\hline 40 & $\mathrm{~N}$-butyl- $\mathrm{f}$-butanamine & 13.17 & 129 & & & & & & & & & & & & & \\
\hline 41 & benzaldehyde & 13.18 & 106 & & 0.02 & 0.01 & 0.01 & 0.03 & & 0.05 & & 0.03 & & 0.02 & 0.02 & \\
\hline 42 & C3-benzene & 13.25 & 120 & & & 0.01 & & & & & 0.03 & 0.06 & 0.12 & & & \\
\hline 43 & C3-benzens & 13.41 & 120 & & & & & & & & 0.04 & & 0.04 & & & \\
\hline 44 & phenol & 13.57 & 94 & & & & & 0.03 & & 0.01 & & 0.02 & & & & \\
\hline 45 & aniline & 13.61 & 93 & & & & & & & & & & & & & \\
\hline 46 & C3-benzene & 13.68 & 120 & & & & & & & 0.01 & & 0.05 & & 0.01 & & \\
\hline 47 & 6-methyi-5-hepten-2-one & 13.69 & 126 & & & & & & & & & & & & & \\
\hline 48 & benzonitrile & 13.72 & 103 & & & & & & & & & & & & & \\
\hline 49 & 1-decene & 13.83 & 140 & & & & & & & & & & & & & \\
\hline 50 & C3-benzene & 13.99 & 120 & & & & & & & & & 0.10 & 0.18 & & & \\
\hline 51 & n-decane & 14.07 & 142 & & & & & & & 0.02 & & 0.03 & 0.02 & 0.01 & & \\
\hline 52 & octanal & 14.09 & 128 & & 0.03 & & & & & & & & & & 0.01 & \\
\hline 53 & dichlorobenzene & 14.40 & 146 & & & & & & & & & & & & & \\
\hline 54 & 4-methyl decane & 14.56 & 156 & & & & & & & & & & & & & \\
\hline 55 & C3-benzene & 14.57 & 120 & & & & & & & & & & & & & \\
\hline 56 & C4-benzene & 14.60 & 134 & & & & & & & & & & 0.06 & & & \\
\hline 57 & 2ethyl hexanol & 14.64 & 130 & & & & 0.04 & 0.09 & & 0.01 & 0.02 & 0.06 & & & & \\
\hline 58 & limonene & 14.75 & 136 & & & & & & & & 0.01 & 0.06 & 0.03 & & & \\
\hline 59 & benzene methanol & 14.77 & 108 & & & & & & & & & & & & & \\
\hline 60 & indan & 14.87 & 118 & & & & & & & & & & & & & \\
\hline 61 & dichlorobenzene & 14.89 & 146 & & & & & & & & & & & & & \\
\hline 62 & N-ethyl-N-(1-methylethyl)-2-propanamine & 15.07 & 129 & & & & & & & & & & & & & \\
\hline 63 & C4-benzene & 15.10 & 134 & & & & & & & & & & 0.03 & 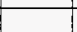 & 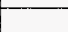 & \\
\hline
\end{tabular}


Air Monitoring Results

Sample number and room number at the top of each data column

\begin{tabular}{|c|c|c|c|c|c|c|c|c|c|c|c|c|c|c|c|c|}
\hline & Compound & & & \begin{tabular}{|c|}
78 \\
$E 403$ \\
\end{tabular} & \begin{tabular}{|c|}
79 \\
$E 403$ \\
\end{tabular} & \begin{tabular}{|c|}
74 \\
$E 405$ \\
\end{tabular} & \begin{tabular}{|c|}
75 \\
$E 405$ \\
\end{tabular} & \begin{tabular}{|c|}
76 \\
$E 408$
\end{tabular} & \begin{tabular}{|c|}
77 \\
$E 408$ \\
\end{tabular} & \begin{tabular}{|c|}
132 \\
$E 408$ \\
\end{tabular} & \begin{tabular}{|c|}
85 \\
$E 409$ \\
\end{tabular} & \begin{tabular}{|c|}
86 \\
$E 409$ \\
\end{tabular} & \begin{tabular}{|c|}
84 \\
$E 411$
\end{tabular} & \begin{tabular}{|c|}
130 \\
$E 411$ \\
\end{tabular} & \begin{tabular}{|c|}
81 \\
$E 412$ \\
\end{tabular} & 8 \\
\hline & Tentative Identification & RT & MW & $s$ & $n$ & ne & sw & ne & sw & & e & w & \begin{tabular}{|l|} 
drway \\
\end{tabular} & & s & 0 \\
\hline 64 & 5-methyl decane & 15.21 & 156 & & & & & & & & & & & & & \\
\hline 65 & 4-methyl decane & 15.29 & 156 & & & & & & & & & & & & & \\
\hline 66 & C4-benzene & 15.30 & 134 & & & & & & & & & & 0.04 & & & \\
\hline 67 & 2-methyl decane & 15.34 & \begin{tabular}{|l|l|}
156 \\
\end{tabular} & & & & & & & & & & & & & \\
\hline 68 & 1,4-dithiane & 15.39 & 120 & & & & & & & & & & & & & \\
\hline 69 & acetophenone & \begin{tabular}{|l|}
15.47 \\
\end{tabular} & 120 & & 0.03 & & & 0.04 & & 0.04 & & & & 0.02 & 0.01 & \\
\hline 70 & C4-benzene & 15.47 & 134 & & & & & & & & & & & & & \\
\hline 71 & 3-methyl decane & 15.48 & 156 & & & & & & & & & & & & & \\
\hline 72 & methyl benzaldehyde & 15.51 & 120 & & & & & & & & & & & & & \\
\hline 73 & C4-benzene & 15.64 & 934 & & & & & & & & & & & & & \\
\hline 74 & C4-benzene & 15.72 & 134 & & & & & & & & & & & & & \\
\hline 75 & A,A-dimethyl benzene methanol & 15.80 & 136 & & & & & & & & & & & & & \\
\hline 76 & C4-benzene & 15.86 & 134 & & & & & & & & & 0.02 & 0.02 & & & \\
\hline 77 & 1-undecene & 15.95 & 154 & & & & & & & & & & & & & \\
\hline 78 & n-undecane & 16.05 & 156 & & 0.02 & trace & & & & 0.02 & & 0.03 & 0.02 & 0.01 & & \\
\hline 79 & nonanal & 16.12 & 142 & & 0.03 & & 0.02 & 0.03 & & & & 0.03 & & & 0.01 & \\
\hline 80 & C4-benzene & 16.24 & 134 & & & & & & & & & & & & & \\
\hline 81 & phosphoric acid, triethyl ester & 16.41 & 182 & & & & & & & & & & & & & \\
\hline 82 & C4-benzene & 16.43 & 134 & & & & & & & & & & & & & \\
\hline 83 & trans methyl decalin & 16.50 & 152 & & & & & & & & & & & & & \\
\hline 84 & C4-benzene & 16.55 & 134 & & & & & & & & & & & & & \\
\hline 85 & cis methyl decalin & 16.74 & 152 & & & & & & & & & & & & & \\
\hline 86 & C5-benzene & 16.79 & 148 & & & & & & & & & & & & & \\
\hline 87 & methyl indan & 17.14 & 132 & & & & & & & & & & & & & \\
\hline 88 & O,O-diethyl-S-ethyl phosphorothioate & 17.16 & 198 & & & & & & & & & & & & & \\
\hline 89 & methyl indan & 17.29 & 132 & & & & & & & & & & & & & \\
\hline 90 & trichlorobenzene & 17.64 & 180 & & & & & & & & & & & & & \\
\hline 91 & 1-dodecene & 17.66 & 168 & & & & & & & & & & & & & \\
\hline 92 & 1-(4-methyiphenyi) ethanone & 17.67 & 134 & & & & & & & & & & & & & \\
\hline 93 & N,N-dibutyl-1-butanamine & 17.72 & 142 & & & & & & & & & & & & & \\
\hline 94 & n-dodecane & 17.80 & 170 & & & & & & & & & & & & & \\
\hline 95 & naphthalene & 17.79 & 128 & & 0.02 & 0.01 & & 0.02 & & 0.01 & 0.01 & 0.05 & 0.04 & 0.00 & & \\
\hline 96 & C5-benzene & 17.87 & 148 & & & & & & & & & & & & & \\
\hline 97 & decanal & 17.90 & 156 & & 0.02 & & 0.02 & 0.04 & & & & 0.02 & & & 0.01 & \\
\hline 98 & benzothiophene & 17.90 & 134 & & & & & & & & & & & & & \\
\hline 99 & 1,4-oxathiane, 4,4-dioxide & 18.35 & 136 & & & & & & & & & & & & & \\
\hline 100 & benzothiazole & 18.40 & 135 & & & & & 0.02 & & & & & & & & \\
\hline 101 & benzene propanenitrile & 18.53 & 131 & & & & & & & & & & & & & \\
\hline 102 & dimethyl indan & 18.80 & 146 & & & & & & & & & & & & & \\
\hline 103 & 3-tetradecene & 18.85 & 196 & & & & & & & & & & & & & \\
\hline 104 & N-butyl-N-nitroso N-butanamine & 18.91 & 158 & & & & & & & & & & & & & \\
\hline 105 & 1-tridecene & 19.26 & 182 & & & & & & & & & & & & & \\
\hline 106 & n-tridecane & 19.38 & 184 & & & & & & & & & & & & & \\
\hline 107 & N,N-dibutyl formamide & 19.51 & 157 & & & & & & & & & & & & & \\
\hline 108 & 2-methyl naphthalene & 19.61 & 142 & & & & & & & & & & & & & \\
\hline 109 & phthalate & 19.80 & 390 & & & & & & & & & & & & & \\
\hline 110 & 1,3-isobenzofurandione & 19.81 & 148 & & & & & & & & & & & & & \\
\hline 111. & 1-methyl naphthalene & 19.91 & 142 & & & & & & & & & & & & & \\
\hline 112 & 1-methyl-4-(propylthio) benzene & 20.37 & 166 & & & & & & & & & & & & & \\
\hline 113 & 1-tetradecene & 20.76 & 196 & & & & & & & & & & & & & \\
\hline 114 & n-tetradecane & 20.86 & 198 & & & & & & & & & & & & & \\
\hline 115 & biphenyl & 20.87 & 154 & & & & & & & & & 0.02 & & & & \\
\hline 116 & 1, 1'-oxy bis(benzene) & 21.17 & 186 & & & & & & & & & 0.03 & & & & \\
\hline 117 & 2,4,6-trichloroaniline & 21.20 & 195 & & & & & & & & & 0.03 & & & & \\
\hline 118 & C2-naphthalene & 21.28 & 156 & & & & & & & & & & & & & \\
\hline 119 & C2-naphthalene & 21.51 & 156 & & & & & & & & & & & & & \\
\hline 120 & C2-naphthalene & 21.56 & 156 & & & & & & & & & & & & & \\
\hline 121 & tribromobenzene & 21.62 & 312 & & & & & & & & & & & & & \\
\hline 122 & 6,10-dimethyl-5,9-undecadien-2-one & 21.65 & 194 & & & & & & & & & & & & & \\
\hline 123 & 2,6-di-t-butyl-2,5-cyclohexadiene-1,4-dione & 22.06 & 220 & & & & & & & & & & & & & \\
\hline 124 & 1-pentadecene & 22.16 & 210 & & & & & & & & & & & & & \\
\hline 125 & n-pentadecane & 22.26 & 212 & & & & & & & & & & & & & \\
\hline 126 & methyl biphenyl & 22.30 & 168 & & & & & & & & & & & & & \\
\hline 127 & 1,2-dihydroacenaphthylene & 22.50 & $\mid 168$ & & & & & & & & & & & & & \\
\hline
\end{tabular}


Air Monitoring Results

Sample number and room number at the top of each data column

\begin{tabular}{|c|c|c|c|c|c|c|c|c|c|c|c|c|c|c|c|c|}
\hline & Compound & & & \begin{tabular}{|c|}
78 \\
$E 403$ \\
\end{tabular} & \begin{tabular}{|c|}
79 \\
$E 403$ \\
\end{tabular} & \begin{tabular}{|c|}
74 \\
5405
\end{tabular} & \begin{tabular}{|c|}
75 \\
$E 405$ \\
\end{tabular} & \begin{tabular}{|c|}
76 \\
$E 408$ \\
\end{tabular} & \begin{tabular}{c|}
77 \\
6408
\end{tabular} & \begin{tabular}{c|}
132 \\
$E 408$
\end{tabular} & \begin{tabular}{|c|}
85 \\
$E 409$ \\
\end{tabular} & \begin{tabular}{|c|}
86 \\
$E 409$ \\
\end{tabular} & \begin{tabular}{|c|}
84 \\
$E 411$ \\
\end{tabular} & \begin{tabular}{|c|}
130 \\
$E 411$
\end{tabular} & \begin{tabular}{|c|}
81 \\
5412
\end{tabular} & 82 \\
\hline & Tentative Identification & RT & MW & $s$ & $n$ & ne & sw & ne & sw & & $e$ & $w$ & drway & & $s$ & 0 \\
\hline 128 & C3-naphthaiene & 22.65 & 170 & & & & & & & & & & & & & \\
\hline 129 & phenyl maleic anhydride & 22.90 & 214 & & & & & & & & & & & & & \\
\hline 130 & dibenzofuran & 22.93 & 168 & & & & & & & & & & & & & \\
\hline 131 & 1-hexadecene & 22.47 & 224 & & & & & & & & & & & & & \\
\hline 132 & n-hexadecane & 23.57 & 226 & & & & & & & & & & & & & \\
\hline 133 & benzenedicarboxylic acid, diethyl ester & 23.64 & 222 & & & & & & & & & & & & & \\
\hline 134 & 1,3-dibromo-2,2-bis(bromomethyl) propane & 24.17 & 384 & & & & & & & & & & & & & \\
\hline 135 & dipheny diazene & 24.23 & 182 & & & & & & & & & & & & & \\
\hline 136 & benzophenone & 24.30 & 482 & & & & & & & & & & & & & \\
\hline 137 & 1-heptadecene & 24.74 & 238 & & & & & & & & & & & & & \\
\hline 138 & n-heptadecane & 24.89 & 240 & & & & & & & & & & & & & \\
\hline 139 & $\mathrm{~N}$-(phenylmethylene) benzamine & 24.83 & 18.1 & & & & & & & & & & & & & \\
\hline 140 & 9H-fluoren-9-one & 25.28 & 180 & & & & & & & & & & & & & \\
\hline 141 & tribromobenzamine & 25.39 & 327 & & & & & & & & & & & & & \\
\hline 142 & trimethyl indan & 25.45 & 160 & & 0.01 & 0.01 & & 0.03 & & 0.01 & & & & & & \\
\hline 143 & dibenzothiophene & 25.58 & 184 & & & & & & & & & & & & & \\
\hline 144 & tetrabromobenzene & 25.86 & 390 & & & & & & & & & & & & & \\
\hline 145 & n-octadecane & 25.88 & 254 & & & & & & & & & & & & & \\
\hline 146 & ohenanthrene/anthracene & 26.37 & 178 & & 0.04 & & & & & & & 0.01 & & & & \\
\hline 147 & methyl dibenzothiophene & 26.98 & 198 & & & & & & & & & & & & & \\
\hline 148 & n-nonadecane & 27.04 & 268 & & & & & & & & & & & & & \\
\hline 149 & 2-methyl anthracene/phenanthrene & 27.48 & 192 & & & & & & & & & & & & & \\
\hline 150 & 1-methyl anthracene/phenanthrene & 27.57 & 192 & & & & & & & & & & & & & \\
\hline 151 & 2,6-dibutyl-2,5-cyclohexadien-1,4-dione & 31.30 & 220 & & & & & & & & & & & & & \\
\hline 152 & Total PCB's & & & & & & & & & & & 1.00 & & & & \\
\hline & & & & & & & & & & & & & & & & \\
\hline & & & & & & & & & & & & & & & & \\
\hline & & & & & & & & & & & & & & & & \\
\hline & & & & & & & & & & & & & & & & \\
\hline & & & & & & & & & & & & & & & & \\
\hline & & & & & & & & & & & & & & & & \\
\hline & & & & & & & & & & & & & & & & \\
\hline & & & & & & & & & & & & & & & & \\
\hline & & & & & & & & & & & & & & & & \\
\hline & & & & & & & & & & & & & & & & \\
\hline & & & & & & & & & & & & & & & & \\
\hline & & & & & & & & & & & & & & & & \\
\hline & & & & & & . & & & & & & & & & & \\
\hline & & & & & & & & & & & & & & & & \\
\hline & 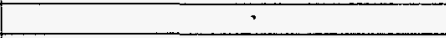 & & & & & & & & & & & & & & & \\
\hline & & & & & & & & & & & & & & & & \\
\hline & & & & & & & & & & & & & & & & \\
\hline & & & & & & & & & & & & & & & & \\
\hline & & & & & & & & & & & & & & & & \\
\hline & & & & & & & & & & & & & & & & \\
\hline & & & & & & & & & & & & & & & & \\
\hline & & & & & & & & & & & & & & & & \\
\hline & & & & & & & & & & & & & & & & \\
\hline & & & & & & & & & & & & & & & & \\
\hline & & & & & & & & & & & & & & & & \\
\hline & & & & & & & & & & & & & & & & \\
\hline & & & & & & & & & & & & & & & & \\
\hline & & & & & & & & & & & & & & & & \\
\hline & & & & & & & & & & & & & & & & \\
\hline & & & & & & & & & & & & & & & & \\
\hline & & & & & & & & & & & & & & & & \\
\hline & & & & & & & & & & & & & & & & \\
\hline & & & & & & & & & & & & & & & & \\
\hline & & & & & & & & & & & & & & & & \\
\hline & & & & & & & & & & & & & & & & \\
\hline & & & & & & & & & & & & & & & & \\
\hline & & & & & & & & & & & & & & & & \\
\hline & 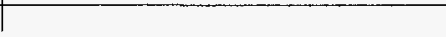 & & & 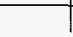 & & & & & & & & & & & & \\
\hline
\end{tabular}


Air Monitoring Results

Sample number and room number at the top of each data column

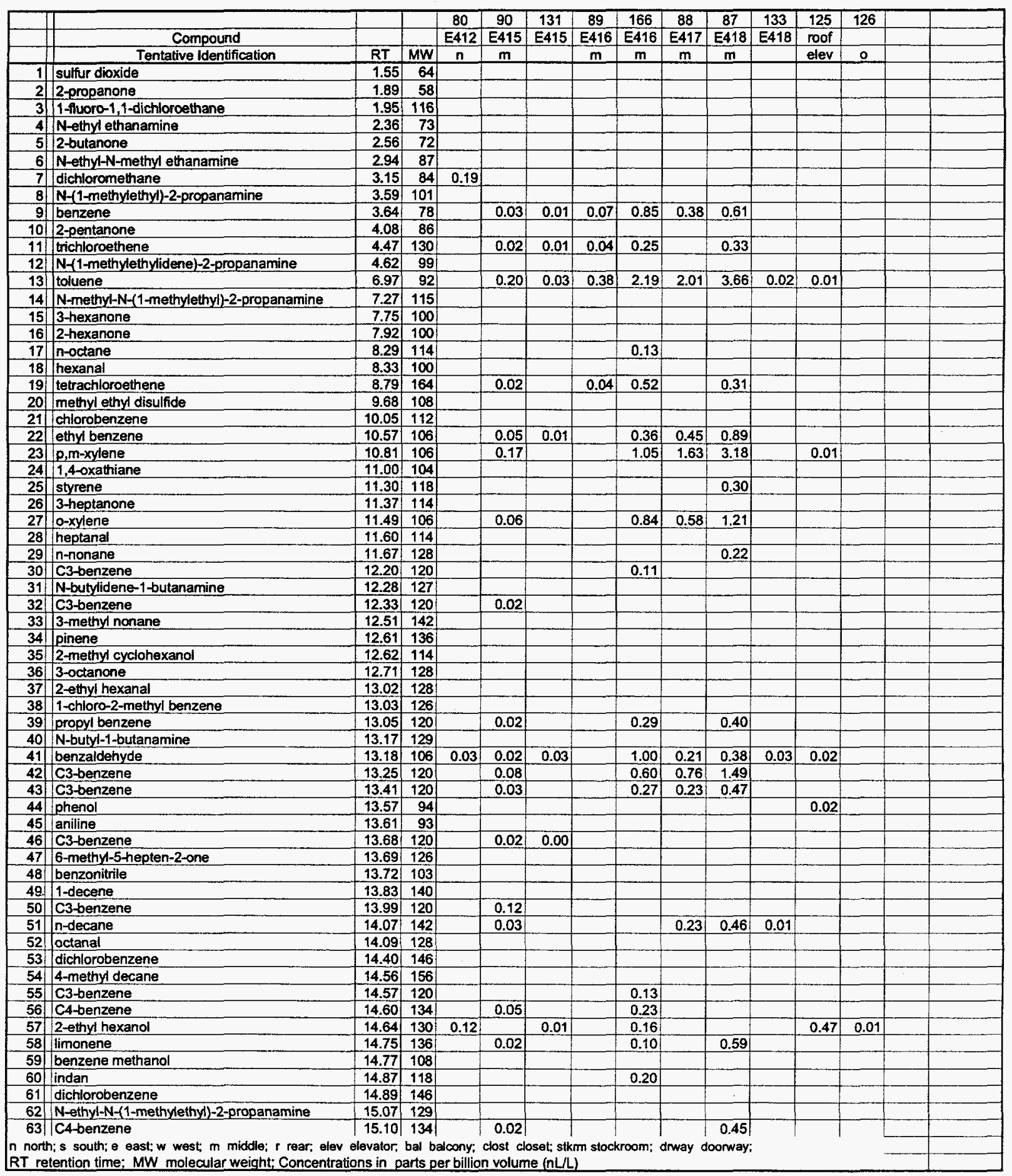


Air Monitoring Results

Sample number and room number at the top of each data column

\begin{tabular}{|c|c|c|c|c|c|c|c|c|c|c|c|c|c|c|c|}
\hline & & & & 80 & 90 & 131 & 89 & 166 & 88 & 87 & 133 & 125 & 126 & & \\
\hline & Compound & & & E412 & E415 & E415 & E416 & E416 & E417 & E418 & E418 & roof & & & \\
\hline & Tentative Identification & RT & MW & $n$ & $m$ & & $m$ & $m$ & $m$ & $\mathrm{~m}$ & & elev & 0 & & \\
\hline 64 & 5-methyl decane & 15.21 & 156 & & & & & & & & & & & & \\
\hline 65 & 4-methyl decane & 15.29 & 156 & & & & & & & & & & & & \\
\hline 66 & C4-benzene & 15.30 & 134 & & 0.03 & & & 0.20 & & 0.37 & & & & & \\
\hline 67 & 2-methyl decane & 15.34 & 156 & & & & & & & & & & & & \\
\hline 68 & 1,4-dithiane & 15.39 & 120 & & & & & & & & & & & & \\
\hline 69 & acetophenone & 15.47 & 120 & 0.02 & & 0.01 & & 0.47 & & 0.26 & 0.03 & 0.02 & 0.01 & & \\
\hline 70 & C4-benzene & 15.47 & 134 & & & & & & & & & & & & \\
\hline 71 & 3-methyl decane & 15.48 & 156 & & & & & & & & & & & & \\
\hline 72 & methyl benzaldehyde & 15.51 & 120 & & & & & & & & & & & & \\
\hline 73 & C4-benzene & 15.64 & 134 & & & & & 0.07 & & & & & & & \\
\hline 74 & C4-benzene & 15.72 & 134 & & & & & & & & & & & & \\
\hline 75 & A,A-dimethyl benzene methanol & 15.80 & 136 & & & & & & & & & & & & \\
\hline 76 & C4-benzene & 15.86 & 134 & & 0.02 & & & 0.32 & & & & & & & \\
\hline 77 & 1-undecene & 15.95 & 154 & & & & & & & & & & & & \\
\hline 78 & n-undecane & 16.05 & 156 & & 0.02 & & & 0.11 & & 0.35 & & & & & \\
\hline 79 & nonanal & 16.12 & 142 & 0.01 & & & & 0.38 & & & 0.02 & 0.03 & 0.01 & & \\
\hline 80 & C4-benzene & 16.24 & 134 & & & & & 0.08 & & & & & & & \\
\hline 81 & phosphoric acid, triethyl ester & 16.41 & 182 & & & & & & & & & & & & \\
\hline 82 & C4-benzene & 16.43 & 134 & & & & & & & & & & & & \\
\hline 83 & trans methyl decalin & 16.50 & 152 & & & & & & & & & & & & \\
\hline 84 & c4-benzene & 16.55 & 134 & & 0.01 & & & & & & & & & & \\
\hline 85 & cis methyl decalin & 16.74 & 152 & & & & & & & & & & & & \\
\hline 86 & C5-benzene & 16.79 & 148 & & & & & & & & & & & & \\
\hline 87 & methyi indan & 17.14 & 132 & & & & & & & & & & & & \\
\hline 88 & 0,0 -diethyl-S-ethyl phosphorothioate & 17.16 & 198 & & & & & & & & & & & & \\
\hline 89 & methyl indan & 17.29 & 132 & & & & & & & & & & & & \\
\hline 90 & trichlorobenzene & 17.64 & 180 & & & & & & & & & & & & \\
\hline 91 & i-dodecene & 17.66 & 168 & & & & & & & & & & & & \\
\hline 92 & 1-(4-methylphenyl) ethanone & 17.67 & 134 & & & & & & & & & & & & \\
\hline 93 & $\mathbf{N}, \mathbf{N}$-dibutyl-1-butanamine & 17.72 & 142 & & & & & & & & & & & & \\
\hline 94 & n-dodecane & 17.80 & 170 & & & & & 0.12 & & & & & & & \\
\hline 95 & naphthalene & 17.79 & 128 & & 0.05 & 0.08 & & 0.23 & 0.41 & 0.87 & & 0.01 & & & \\
\hline 96 & C5-benzene & 17.87 & 148 & & & & & & & & & & & & \\
\hline 97 & decanal & 17.90 & 156 & & & & & 0.32 & & & & 0.02 & & & \\
\hline 98 & benzothiophene & 17.90 & 134 & & & & & & & & & & & & \\
\hline 99 & 1,4-oxathiane, 4,4-dioxide & 18.35 & 136 & & & & & & & & & & & & \\
\hline 100 & benzothiazole & 18.40 & 135 & & & & & & & & & & & & \\
\hline 101 & benzene propanenitrile & 18.53 & 131 & & & & & & & & & & & & \\
\hline 102 & dimethyl indan & 18.80 & 146 & & & & & & & & & & & & \\
\hline 103 & 3-tetradecene & 18.85 & 196 & & & & & & & & & & & & \\
\hline 104 & $\mathrm{~N}$-butyl-N unitroso $\mathrm{N}$-butanamine & 18.91 & 158 & & & & & & & & & & & & \\
\hline 105 & 1-tridecene & 19.26 & 182 & & & & & & & & & & & & \\
\hline 106 & n-tridecane & 19.38 & 184 & & & & & 0.03 & & & & & & & \\
\hline 107 & N,N-dibutyl formamide & 19.51 & 157 & & & & & & & & & & & & \\
\hline 108 & 2-methyl naphthalene & 19.61 & 142 & & 0.01 & & & 0.05 & 0.13 & 0.23 & & & & & \\
\hline 109 & phthalate & 19.80 & 390 & & & & & & & & & 0.04 & & & \\
\hline 110 & 1,3-isobenzofurandione & 19.81 & 148 & & & & & 0.04 & & & & 0.05 & & & \\
\hline 111 & 1-methyl naphthalene & 19.91 & 142 & & & & & 0.07 & & 0.18 & & & & & \\
\hline 112 & 1-methy-4-(propylthio) benzene & 20.37 & 166 & & & & & & & & & & & & \\
\hline 113 & 1-tetradecene & 20.76 & 196 & & & & & & & & & & & & \\
\hline 114 & n-tetradecane & 20.86 & 198 & & & & & & & & & & & & \\
\hline 115 & biphenyl & 20.87 & 154 & & 0.01 & & & 0.05 & & & & & & & \\
\hline 116 & $1,1^{1}$-oxy bis(benzene) & 21.17 & 186 & & 0.02 & & & 0.07 & & 0.15 & & & & & \\
\hline 117 & 2,4,6-trichloroaniline & 21.20 & 195 & & & & & 0.05 & & 0.23 & & & & & \\
\hline 118 & C2-naphthalene & 21.28 & 156 & & & & & & & & & & & & \\
\hline 119 & C2-naphthalene & 21.51 & 156 & & & & & 0.06 & & 0.06 & & & & & \\
\hline 120 & C2-naphthalene & 21.56 & 156 & & & & & & & & & & & & \\
\hline 121 & tribromobenzene & 21.62 & 312 & & & & & & & & & & & & \\
\hline 122 & 6,10-dimethyl-5,9-undecadien-2-one & 21.65 & 194 & & & & & & & & & & & & \\
\hline 123 & 2,6-di-t-butyt-2,5-cyclohexadiene-1,4-dione & 22.06 & 220 & & & & & & & & & & & & \\
\hline 124 & 1-pentadecene & 22.16 & 210 & & & & & & & & & & & & \\
\hline 125 & n-pentadecane & 22.26 & 212 & & & & & & & & & & & & \\
\hline 126 & methyl biphenyl & 22.30 & 168 & & & & & & & & & & & & \\
\hline 127 & 1,2-dihydroacenaphthylene & 22.50 & 168 & & & & & & & & & & & & \\
\hline
\end{tabular}


Air Monitoring Results

Sample number and room number at the top of each data column

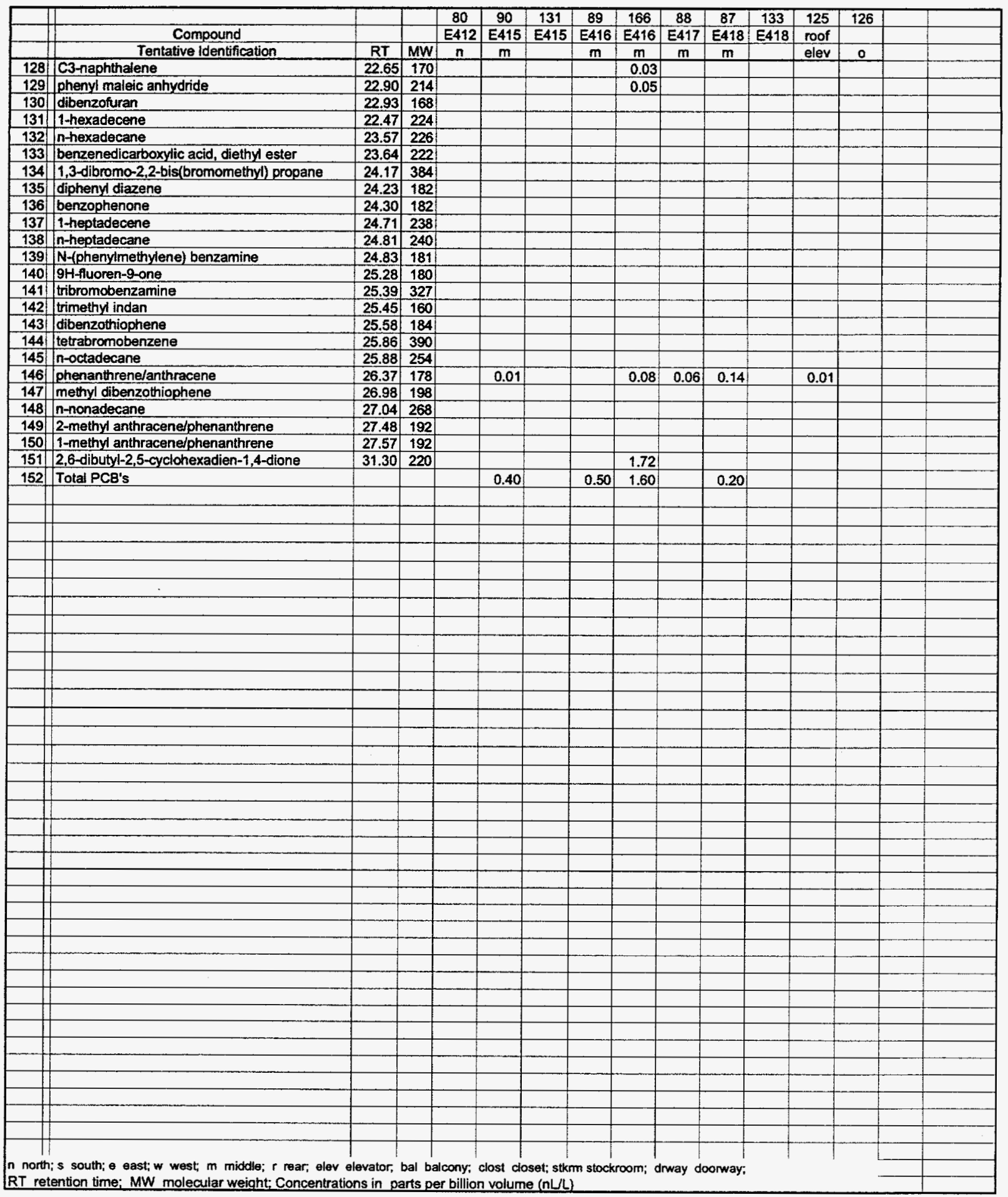




\begin{tabular}{|c|c|c|c|c|c|c|c|c|c|c|c|c|c|c|c|}
\hline & & & & 83 & 98 & 99 & 96 & 97 & 95 & 91 & 92 & 175 & 176 & 174 & 177 \\
\hline & Compound & & & F104 & G110 & & G118 & G120 & G120 & 1101 & & 1101 & & 1101 & 1101 \\
\hline & Tentative Identification & RT & MW & Stkrm & $m$ & 0 & $m$ & e & $w$ & $\mathrm{~m}$ & 0 & $\mathrm{~m}$ & 0 & sump & sump \\
\hline 1 & sulfur dioxide & 1.55 & 64 & & & & & & & 0.01 & & & & & \\
\hline 2 & 2-propanone & 1.89 & 58 & & & & & & & & & & & & \\
\hline 3 & 1-fluoro-1,1-dichloroethane & 1.95 & 116 & & & & & & & & & & & & \\
\hline 4 & T/ Nethyl ethanamine & 2.36 & 73 & & & & & & & & & & & & \\
\hline 5 & 2-butanone & 2.56 & 72 & & & & & & & & & & & & \\
\hline 6 & N-ethyl-N-methyl ethanamine & 2.94 & 87 & & & & & & & & & & & & \\
\hline 7 & dichloromethane & 3.15 & 84 & & & & & & & & & & & & \\
\hline 8 & N-(1-methylethy)-2-propanamine & 3.59 & 101 & & & & & & & & & & & & \\
\hline 9 & benzene & 3.64 & 78 & & 0.01 & & & & 0.03 & & & 0.36 & 0.52 & 0.38 & 0.31 \\
\hline 10 & 2-pentanone & 4.08 & 86 & & & & & & & & & & & & \\
\hline 11 & trichloroethene & 4.47 & 130 & & & & & & & & & 0.03 & $t$ & 0.52 & 0.30 \\
\hline 12 & $\mathrm{~N}-(1-$ methylethylidene)-2-propanamine & 4.62 & 99 & & & & & & & & & & & & \\
\hline 13 & toluene & 6.97 & 92 & 0.24 & 0.09 & 0.06 & 0.08 & 0.10 & 0.11 & 0.04 & 0.07 & 1.23 & 0.82 & 0.97 & 0.48 \\
\hline 14 & $\mathrm{~N}$-methyl-N-(1-methylethyl)-2-propanamine & 7.27 & 115 & & & & & & & & & & & & \\
\hline 15 & 3-hexanone & 7.75 & 100 & & & & & & & & & & & & \\
\hline 16 & 2-hexanone & 7.92 & 100 & & & & & & & & & & & & \\
\hline 17 & n-octane & 8.29 & 114 & & & & & & & & & & & & \\
\hline 18 & hexanal & 8.33 & 100 & & & & & & & & & 0.10 & & 0.30 & 0.27 \\
\hline 19 & tetrachloroethene & 8.79 & 164 & & 0.01 & & 0.02 & 0.01 & 0.01 & & & & & & \\
\hline 20 & methyl ethyl disulfide & 9.68 & 108 & & & & & & & & & & & & \\
\hline 21 & chlorobenzene & 10.05 & 112 & & & & & & & & & & & & \\
\hline 22 & ethyl benzene & 10.57 & 106 & & 0.02 & 0.02 & 0.01 & 0.02 & 0.02 & 0.01 & 0.01 & 0.24 & 0.12 & 0.16 & 0.12 \\
\hline 23 & p,m-xylene & 10.81 & 106 & 0.13 & 0.05 & 0.06 & 0.05 & 0.06 & 0.06 & 0.02 & 0.03 & 0.32 & 0.16 & 0.31 & 0.16 \\
\hline 24. & 1,4-oxathiane & 11.00 & 104 & & & & & & & & & & & & \\
\hline 25 & styrene & 11.30 & 118 & & 0.01 & & 0.01 & 0.01 & 0.01 & & & & & & \\
\hline 26 & 3-heptanone & 11.37 & 114 & & & & & & & & & 0.06 & & & \\
\hline 27 & o-xylene & 11.49 & 106 & & 0.02 & 0.03 & 0.02 & 0.02 & 0.02 & 0.01 & 0.01 & 0.28 & 0.15 & 0.23 & \\
\hline 28 & heptanal & 11.60 & 114 & & & & & & & & & & & & \\
\hline 29 & | n-nonane & 11.67 & 128 & & 0.01 & & 0.01 & & 0.01 & 0.02 & & & & & 0.23 \\
\hline 30 & C3-benzene & 12.20 & 120 & & & & & & & & & & & & \\
\hline 31 & $\mathrm{~N}$-butylidene- 1 -butanamine & 12.28 & 127 & & & & & & & & & & & & \\
\hline 32 & C3-benzene & 12.33 & 120 & & & & & & & & & & & & \\
\hline 33 & 3-methyl nonane & 12.51 & 142 & & & & & & & & & & & & \\
\hline 34 & pinene & 12.61 & 136 & & 0.01 & & 0.01 & 0.02 & 0.02 & 0.01 & & 0.09 & & & \\
\hline 35 & 2-methyl cyciohexanol & 12.62 & 114 & & & & & & & 0.02 & & 0.28 & & & \\
\hline 36 & 3-octanone & 12.71 & 128 & & & & & & & & & & & & \\
\hline 37 & 2-ethyi hexanal & 13.02 & 128 & & & & & & & & & & & & \\
\hline 38 & 1-chloro-2-methyl benzene & 13.03 & 126 & & & & & & & & & & & & \\
\hline 39 & propyl benzene & 13.05 & 120 & & & & & & & & & 0.13 & & & \\
\hline 40 & N-butyl-1-butanamine & 13.17 & 129 & & & & & & & & & & & & \\
\hline 41 & benzaldehyde & 13.18 & 106 & & 0.07 & 0.08 & 0.05 & 0.05 & 0.04 & 0.02 & 0.05 & 1.50 & 1.90 & 1.10 & 0.56 \\
\hline 42 & C3-benzene & 13.25 & 120 & 0.07 & & 0.02 & 0.03 & 0.03 & 0.03 & 0.01 & 0.01 & 0.22 & & & \\
\hline 43 & C3-benzene & 13.41 & 120 & & & & 0.02 & & 0.01 & & & & & & \\
\hline 44 & phenol & 13.57 & 94 & & 0.03 & 0.02 & 0.02 & 0.03 & & 0.02 & 0.02 & & 0.67 & & 0.16 \\
\hline 45 & aniline & 13.61 & 93 & & & & & & & & & & & & \\
\hline 46 & C3-benzene & 13.68 & 120 & & 0.03 & & & 0.02 & & & & & & & \\
\hline 47 & 6-methyt-5 hepten-2-one & 13.69 & 126 & & & & & & & & & & & & \\
\hline 48 & benzonitrile & 13.72 & 103 & & & & & & & & & 0.20 & 0.11 & & 0.10 \\
\hline 49 & 1-decene & 13.83 & 140 & & & & & & & & & & & & \\
\hline 50 & C3-benzene & 13.99 & 120 & & & & & & 0.07 & & & & & & \\
\hline 51 & n-decane & 14.07 & 142 & & & & 0.03 & & 0.02 & 0.02 & & & & & \\
\hline 52 & octanal & 14.09 & 128 & & & 0.01 & 0.04 & 0.03 & & 0.02 & 0.01 & & & & 0.19 \\
\hline 53 & dichlorobenzene & 14.40 & 146 & & & & 0.01 & & 0.01 & & & & & & \\
\hline 54 & 4-methyt decane & 14.56 & 156 & & & & & & & & & & & & \\
\hline 55 & C3-benzene & 14.57 & 120 & & & & & & & & & & & & \\
\hline 56 & C4-benzene & 14.60 & 134 & & & & & & & & & & & & \\
\hline 57 & 2-ethyl hexanol & 14.64 & 130 & & 0.04 & 0.01 & 0.08 & 0.05 & 0.05 & 0.03 & & 0.49 & & & \\
\hline 58 & limonene & 14.75 & 136 & 0.23 & & & 0.02 & 0.04 & 0.02 & 0.01 & 0.01 & & & & \\
\hline 59. & 1. benzene methanol & 14.77 & 108 & & 0.02 & & & & & & & & & & \\
\hline 60 & indan & 14.87 & 118 & & & & & & & & & & & & \\
\hline 61 & dichilorobenzene & 14.89 & 146 & & & & & & & & & & & 0.18 & 0.11 \\
\hline 62 & $\mid \mathrm{N}$-ethyi-N-(1-methylethy)-2-propanamine & 15.07 & 129 & & & & & & & & & & & & \\
\hline 63 & C4-benzene & 15.10 & $\mid \mathbf{1 3 4}$ & & & & & & & 0.02 & & & & & \\
\hline
\end{tabular}




\begin{tabular}{|c|c|c|c|c|c|c|c|c|c|c|c|c|c|c|c|}
\hline & & & & 83 & 98 & 99 & 96 & 97 & 95 & 91 & 92 & 175 & 176 & 174 & 177 \\
\hline & Compound & & & F104 & G110 & & G118 & G120 & G120 & I101 & & 1101 & & 1101 & 1101 \\
\hline & Tentative Identification & RT & MW & Stkrm & $\mathrm{m}$ & 0 & m & e & w & $\mathbf{m}$ & 으 & $\mathbf{m}$ & - & sump & sump \\
\hline 64 & 5-methyl decane & 15.21 & 156 & & & & & & & & & & & & \\
\hline 65 & 4-methyl decane & 15.29 & 156 & & & & & & & & & & & & \\
\hline 66 & C4-benzene & 15.30 & 134 & & & & & & & 0.04 & & & & & \\
\hline 67. & 2-methyl decane & 15.34 & 156 & & & & & & & & & & & & \\
\hline 68 & 1,4-dithiane & 15.39 & 120 & & & & & & & & & & & & \\
\hline 69 & acetophenone & 15.47 & 120 & & 0.03 & 0.04 & 0.04 & 0.05 & & 0.03 & 0.05 & 0.77 & 1.35 & 0.48 & 0.45 \\
\hline 70 & C4-benzene & 15.47 & 134 & & & & & & & & & & & & \\
\hline 71 & 3-methyl decane & 15.48 & 156 & & & & & & & & & & & & \\
\hline 72 & methyl benzaldehyde & 15.51 & 120 & & & & & & & & & & & & \\
\hline 73 & C4-benzene & 15.64 & 134 & & & & & & & & & & & & \\
\hline 74 & C4-benzene & 15.72 & 134 & & & & & & & & & & & & \\
\hline 75 & A,A-dimethyl benzene methanol & 15.80 & 136 & & & & & & & & & & & & \\
\hline 76. & C4-benzene & 15.86 & 134 & & & & & & & & & & & & \\
\hline 77 & 1-undecene & 15.95 & 154 & & & & & & & & & & & & \\
\hline 78 & n-undecane & 16.05 & 156 & & 0.01 & & 0.03 & 0.02 & 0.02 & 0.01 & & 0.09 & & & 0.12 \\
\hline 79 & nonanal & 16.12 & 142 & & 0.03 & 0.02 & 0.04 & 0.04 & 0.03 & 0.03 & 0.02 & 0.19 & 0.25 & 0.38 & 0.12 \\
\hline 80 & C4-benzene & 16.24 & 134 & & & & & & & & & & & & \\
\hline 81 & phosphoric acid, triethyl ester & 16.41 & 182 & & & & & & & & & & & & \\
\hline 82 & C4-benzene & 16.43 & 134 & & & & & & & & & & & & \\
\hline 83 & trans methyl decalin & 16.50 & 152. & & & & & & & & & & & & \\
\hline 84 & C4-benzene & 16.55 & 134 & & & & & & & & & & & & \\
\hline 85 & cis methyl decalin & 16.74 & 152 & & & & & & & & & & & & \\
\hline 86 & C5-benzene & 16.79 & 148 & & & & & & & & & & & & \\
\hline 87 & methyl indan & 17.14 & 132 & & & & & & & & & & & & \\
\hline 88 & 0,0-diethyl-S-ethyl phosphorothioate & 17.16 & 198 & & & & & & & & & & & & \\
\hline 89 & methyl indan & 17.29 & 132 & & & & & & & & & & & & \\
\hline 90 & trichlorobenzene & 17.64 & 180 & & & & & & & & & & & & \\
\hline 91 & 1-dodecene & 17.66 & 168. & & & & & & & & & & & & \\
\hline 92 & 1-(4-methylphenyl) ethanone & 17.67 & 134 & & & & & & & & & & & & \\
\hline 93 & $\mathrm{~N}, \mathrm{~N}$-dibutyl-1-butanamine & 17.72 & 142 & & & & & & & & & & & & \\
\hline 94 & n-dodecane & 17.80 & 170 & & & & & & & & & & & & \\
\hline 95 & naphthalene & 17.79 & 128 & 0.08 & 0.04 & 0.01 & 0.09 & 0.07 & 0.07 & 0.02 & 0.01 & 0.14 & & 0.06 & \\
\hline 96 & C5-benzene & 17.87 & 148. & & & & & & & & & & & & \\
\hline 97 & decanal & 17.90 & 156 & & 0.01 & 0.02 & 0.02 & 0.02 & & 0.02 & 0.03 & 0.12 & 0.07 & 0.15 & \\
\hline 98. & benzothiophene & 17.90 & 134 & & & & & & & & & & & & \\
\hline 99 & 1,4-oxathiane, 4,4-dioxide & 18.35 & 136 & & & & & & & & & & & & \\
\hline 100 & benzothiazole & 18.40 & 135 & & & & & & & 0.01 & & & & & 0.10 \\
\hline 101 & benzene propanenitrile & 18.53 & 131 & & & & & & & & & & & & \\
\hline 102 & dimethyl indan & 18.80 & 146 & & & & & & & & & & & & \\
\hline 103 & 3-tetradecene & 18.85 & 196 & & & & & & & & & & & & \\
\hline 104 & $\mathrm{~N}$-butyl-N-nitroso $\mathrm{N}$-butanamine & 18.91 & 158 & & & & & & & & & & & & \\
\hline 105 & 1-tridecene & 19.26 & 182. & & & & & & & & & & & & \\
\hline 106 & n-tridecane & 19.38 & 184 & 0.02 & 0.01 & & & 0.01 & & & & 0.05 & & & \\
\hline 107 & N,N-dibutyl formamide & 19.51 & 157 & & & & & & & & & & & & \\
\hline 108 & 2-methyl naphthalene & 19.61 & 142 & & 0.02 & & 0.03 & 0.02 & 0.02 & 0.01 & & 0.04 & & 0.08 & \\
\hline 109 & phthalate & 19.80 & 390 & & & & & & & & & & & & \\
\hline 110 & 1,3-isobenzofurandione & 19.81 & 148 & & & & & & & & & & & & 0.03 \\
\hline 111 & 1-methyl naphthalene & 19.91 & 142 & & & & 0.01 & 0.01 & & 0.00 & & & & 0.19 & \\
\hline 112 & 1-methyl-4-(propylthio) benzene & 20.37 & 166 & & & & & & & & & & & & \\
\hline 113 & 1-tetradecene & 20.76 & 196 & & & & & 0.01 & & & & & & & \\
\hline 114 & n-tetradecane & 20.86 & 198 & 0.05 & & & & & & 0.01 & & 0.05 & & 0.06 & 0.02 \\
\hline 115 & bipheny & 20.87 & 154 & & 0.01 & & 0.01 & 0.02 & 0.01 & & & & & & \\
\hline 116 & 1,1'-oxy bis(benzene) & 21.17 & 186 & & & & & & & & & & & & \\
\hline 117 & 2,4,6-trichloroaniline & 21.20 & 195 & & & & & & & & & 0.03 & & & \\
\hline 118 & C2-naphthalene & 21.28 & 156 & & & & 0.01 & & & & & & & & 0.05 \\
\hline 119 & C2-napinthalene & 21.51 & 156 & & & & & 0.01 & & & & 0.12 & & 0.35 & 0.09 \\
\hline 120 & C2-naphthalene & 21.56 & 156 & & & & & & & & & & & & \\
\hline 121 & tribromobenzene & 21.62 & 312 & & & & & & & & & & & & \\
\hline 122 & 6,10-dimethyl-5,9-undecadien-2-one & 21.65 & 194 & & & & & & & & & & & & \\
\hline 123 & 2,6-di-t-butyl-2,5-cyclohexadiene-1,4-dione & 22.06 & 220 & & & & & & & & & & & & \\
\hline 124 & 1-pentadecene & 22.16 & 210 & & & & & & & 0.01 & & & & & \\
\hline 125 & n-pentadecane & 22.26 & 212 & 0.04 & 0.01 & & & 0.01 & & & & 0.04 & & & \\
\hline 126 & methyl biphenyl & 22.30 & 168 & & & & & & & & & & & & \\
\hline 127 & 1,2-dihydroacenaphthylene & 22.50 & 168 & 3 & 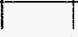 & & & & & & & & & & \\
\hline
\end{tabular}


Air Monitoring Results

Sample number and room number at the top of each data column

\begin{tabular}{|c|c|c|c|c|c|c|c|c|c|c|c|c|c|c|c|}
\hline & & & & 83 & 98 & 99 & 96 & 97 & 95 & 91 & 92 & 175 & 176 & 174 & $\frac{177}{1101}$ \\
\hline & Compound & & & F104 & G110 & & G118 & G120 & G120 & 1101 & & 1901 & & 1101 & 1101 \\
\hline & Tentative Identification & RT & MW & Stkrm & m & 0 & m & e & $\mathbf{w}$ & m & o & $\mathrm{m}$ & 0 & sump & sump \\
\hline 128 & C3-naphthalene & 22.65 & 170 & & & & & & & & & 0.15 & & 0.29 & \\
\hline 129 & phenyl maleic anhydride & 22.90 & 214 & & & & & & & & & & 0.13 & & 0.09 \\
\hline 130 & dibenzofuran & 22.93 & 168 & & & & 0.01 & & 0.01 & & & & & & \\
\hline 131 & 1-hexadecene & 22.47 & 224 & & & & & & & & & & & & \\
\hline 132 & n-hexadecane & 23.57 & 226 & 0.03 & & & & & & & & & & & \\
\hline 133 & benzenedicarboxylic acid, diethyl ester & 23.64 & 222 & & & & & & & & & & & & 0.05 \\
\hline 134 & 1,3-dibromo-2,2-bis(bromomethyl) propane & 24.17 & 384 & & & & & & & & & & & & \\
\hline 135 & diphenyl diazene & 24.23 & 182 & & & & & & & & & & & & \\
\hline 136 & benzophenone & 24.30 & 182 & & & & & & & & & 0.08 & 0.08 & 0.28 & \\
\hline 137 & 1-heptadecene & 24.71 & 238 & & & & & & & & & & & & \\
\hline 138 & n-hepladecane & 24.81 & 240 & 0.02 & & & & & & & & & & & \\
\hline 139 & $\mathrm{~N}$-(phenyimethylene) benzamine & 24.83 & 481 & & & & & & & & & & & & \\
\hline 140 & 9H-fluoren-9-one & 25.28 & 180 & & & & & & & & & & & & \\
\hline 141 & tribromobenzamine & 25.39 & 327 & & & & & & & & & & & & \\
\hline 142 & trimethyl indan & 25.45 & 160 & & 0.01 & & & 0.01 & & & 0.01 & & & & \\
\hline 143 & dibenzothiophene & 25.58 & 184 & & & & & & & & & & & & \\
\hline 144 & tetrabromobenzene & 25.86 & 390 & & & & & & & & & & & & \\
\hline 145 & n-octadecane & 25.88 & 254 & & & & & & & & & & & & \\
\hline 146 & phenanthrene/anthracene & 26.37 & 178 & & 0.01 & & 0.01 & 0.01 & 0.01 & & & & & & \\
\hline 147 & methyl dibenzothiophene & 26.98 & 198 & & & & & & & & & & & & \\
\hline 148 & n-nonadecane & 27.04 & 268 & & & & & & & & & & & & \\
\hline 149 & 2-methyl anthracene/phenanthrene & 27.48 & 192 & & & & & & & & & & & & \\
\hline 150 & 1-methyl anthracene/phenanthrene & 27.57 & 192 & & & & & & & & & & & & \\
\hline 151 & 2,6-dibutyl-2,5-cyclohexadien-1,4-dione & 31.30 & 220 & & & & & & & & & 0.38 & 1.36 & & 0.31 \\
\hline 152 & Total PCB's & & & & & & & & & & & & & & \\
\hline & & & & & & & & & & & & & & & \\
\hline & & & & & & & & & & & & & & & \\
\hline & & & & & & & & & & & & & & & \\
\hline & & & & & & & & & & & & & & & \\
\hline & & & & & & & & & & & & & & & \\
\hline & & & & & & & & & & & & & & & \\
\hline & & & & & & & & & & & & & & & \\
\hline & & & & & & & & & & & & & & & \\
\hline & 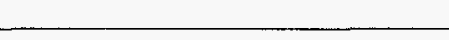 & & & & & & & & & & & & & & \\
\hline & & & & & & & & & & & & & & & \\
\hline & & & & & & & & & & & & & & & \\
\hline & & & & & & & & & & & & & & & \\
\hline & & & & & & & & & & & & & & & \\
\hline & & & & & & & & & & & & & & & \\
\hline & & & & & & & & & & & & & & & \\
\hline & & & & & & & & & & & & & & & \\
\hline & & & & & & & & & & & & & & & \\
\hline & & & & & & & & & & & & & & & \\
\hline & & & & & & & & & & & & & & & \\
\hline & & & & & & & & & & & & & & & \\
\hline & & & & & & & & & & & & & & & \\
\hline & & & & & & & & & & & & & & & \\
\hline & & & & & & & & & & & & & & & \\
\hline & & & & & & & & & & & & & & & \\
\hline & & & & & & & & & & & & & & & \\
\hline & & & & & & & & & & & & & & & \\
\hline & & & & & & & & & & & & & & & \\
\hline & & & & & & & & & & & & & & & \\
\hline & & & & & & & & & & & & & & & \\
\hline & & & & & & & & & & & & & & & \\
\hline & & & & & & & & & & & & & & & \\
\hline & & & & & & & & & & & & & & & \\
\hline & & & & & & & & & & & & & & & \\
\hline & & & & & & & & & & & & & & & \\
\hline & 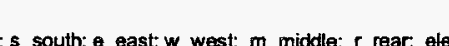 & & & & & & & & 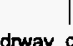 & 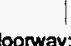 & & & & & \\
\hline
\end{tabular}

